\title{
DEVELOPMENT OF A LOW-COST INFERENTIAL NATURAL GAS ENERGY FLOW RATE PROTOTYPE RETROFIT MODULE
}

\section{TOPICAL REPORT}

For the reporting period:

September 2000 - January 2002

Prepared by:

E. Kelner

T. E. Owen

D. L. George

A. Minachi

M. G. Nored

C. J. Schwartz

March 2004

Prepared for:

GAS RESEARCH INSTITUTE

GRI Contract No. 5097-270-3937

U.S. DEPARTMENT OF ENERGY

DOE Cooperative Agreement No. DE-FC21-96MC33033

GRI Project Manager

Charles E. French

Pipeline Operations

\author{
DOE Technical Monitor \\ James Ammer \\ Gas Supply Projects
}

Submitted by:

SOUTHWEST RESEARCH INSTITUTE ${ }^{\circledR}$

Mechanical and Fluids Engineering Division

6220 Culebra Road

San Antonio, Texas, USA 78238-5166 
This page is intentionally blank. 


\section{Disclaimer}

This report was prepared as an account of work sponsored by an agency of the United States Government. Neither the United States Government nor any agency thereof, nor any of their employees, makes any warranty, express or implied, or assumes any legal liability or responsibility for the accuracy, completeness, or usefulness of any information, apparatus, product, or process disclosed, or represents that its use would not infringe privately owned rights. Reference herein to any specific commercial product, process, or service by trade name, trademark, manufacturer, or otherwise does not necessarily constitute or imply its endorsement, recommendation, or favoring by the United States Government or any agency thereof. The views and opinions of authors expressed herein do not necessarily state or reflect those of the United States Government or any agency thereof.

This report was prepared by Southwest Research Institute (SwRI) as an account of contracted work sponsored by the Gas Research Institute (GRI). Neither SwRI, GRI, members of these companies, nor any person acting on their behalf:

a. Makes any warranty or representation, expressed or implied, with respect to the accuracy, completeness, or usefulness of the information contained in this report, or that the use of any apparatus, methods, or process disclosed in this report may not infringe upon privately owned rights; or

b. Assumes any liability with respect to the use of, or for damages resulting from the use of, any information, apparatus, method, or process disclosed in this report.

References to trade names or specific commercial products, commodities, or services in this report does not represent or constitute an endorsement, recommendation, or favoring by SwRI or GRI of the specific commercial product, commodity, or service. 
This page is intentionally blank. 


\title{
DEVELOPMENT OF A LOW-COST INFERENTIAL NATURAL GAS ENERGY FLOW RATE PROTOTYPE RETROFIT MODULE
}

\author{
E. Kelner \\ T. E. Owen \\ D. L. George \\ A. Minachi \\ M. G. Nored \\ C. J. Schwartz \\ SOUTHWEST RESEARCH INSTITUTE ${ }^{\circledR}$ \\ Mechanical and Fluids Engineering Division \\ 6220 Culebra Road \\ San Antonio, Texas, USA 78238-5166
}

\begin{abstract}
In 1998, Southwest Research Institute ${ }^{\circledR}$ began a multi-year project co-funded by the Gas Research Institute (GRI) and the U.S. Department of Energy. The project goal is to develop a working prototype instrument module for natural gas energy measurement. The module will be used to retrofit a natural gas custody transfer flow meter for energy measurement, at a cost an order of magnitude lower than a gas chromatograph.

Development and evaluation of the prototype retrofit natural gas energy flow meter in 2000-2001 included: (1) evaluation of the inferential gas energy analysis algorithm using supplemental gas databases and anticipated worst-case gas mixtures; (2) identification and feasibility review of potential sensing technologies for nitrogen diluent content; (3) experimental performance evaluation of infrared absorption sensors for carbon dioxide diluent content; and (4) procurement of a custom ultrasonic transducer and redesign of the ultrasonic pulse reflection correlation sensor for precision speed-of-sound measurements. A prototype energy meter module containing improved carbon dioxide and speed-of-sound sensors was constructed and tested in the GRI Metering Research Facility at SwRI. Performance of this module using transmissionquality natural gas and gas containing supplemental carbon dioxide up to $9 \mathrm{~mol} \%$ resulted in gas energy determinations well within the inferential algorithm worst-case tolerance of $\pm 2.4 \mathrm{Btu} / \mathrm{scf}$ (nitrogen diluent gas measured by gas chromatograph). A two-week field test was performed at a gas-fired power plant to evaluate the inferential algorithm and the data acquisition requirements needed to adapt the prototype energy meter module to practical field site conditions.
\end{abstract}


This page is intentionally blank. 


\section{Table of Contents}

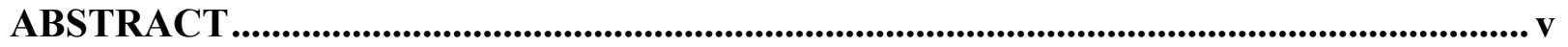

RESEARCH SUMMARY ................................................................................................................vii

LIST OF FIGURES ........................................................................................................

LIST OF TABLES ..............................................................................................................................

$1.0 \quad$ Introduction ............................................................................................................................ 1

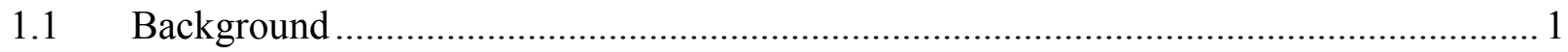

$1.2 \quad$ Project Tasks and Goals, 2000-2001 ……………................................................

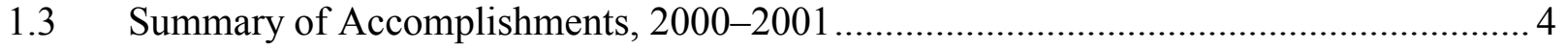

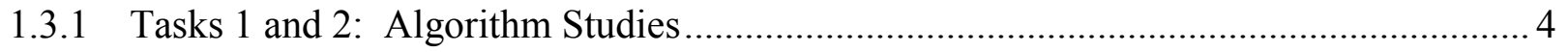

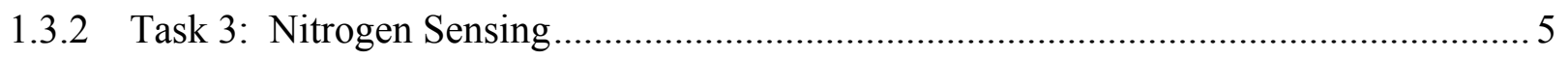

1.3.3 Task 4: Thermal Effects Analysis and Design Improvements........................................ 5

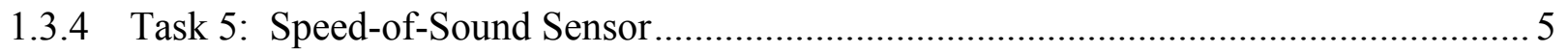

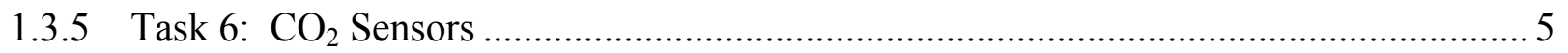

1.3.6 Task 7: Laboratory and Field Tests ...................................................................... 5

2.0 Evaluation and Improvement of Inferential Algorithm.................................................... 7

2.1 Review of Existing Energy Meter Algorithm ............................................................ 7

2.2 Validation of Existing Algorithm Using Independent Gas Databases............................. 11

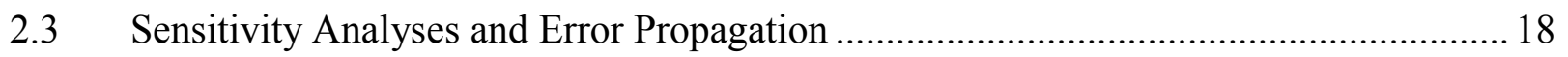

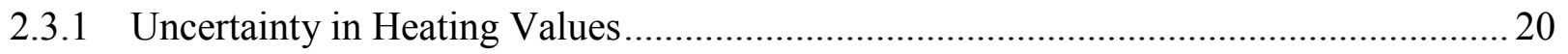

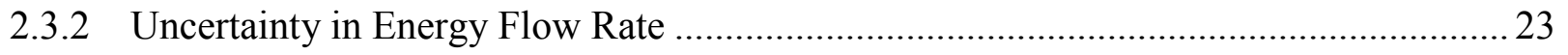

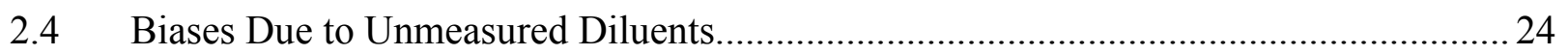

2.5 Extension of Algorithm to Higher Diluent Contents ..................................................2

3.0 Module Improvements.............................................................................................................31

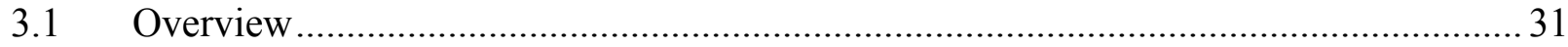

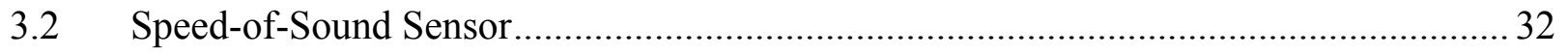

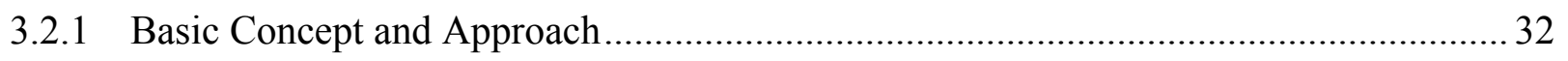

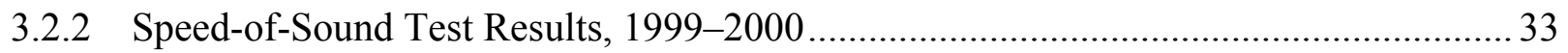

3.2.2.1 Speed-of-sound measurement methods ........................................................... 34

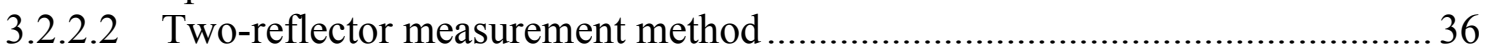




\section{Table of Contents (continued)}

3.2.3 Evaluation of Speed-of-Sound Measurement Characteristics ................................... 38

3.2.3.1 Ultrasonic beam diffraction ................................................................. 38

3.2.3.2 Near-field diffraction and phase error....................................................... 39

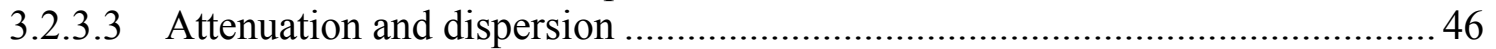

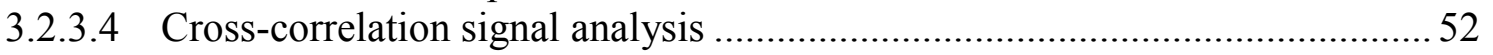

3.2.3.5 Speed-of-sound sensor bench tests ........................................................... 58

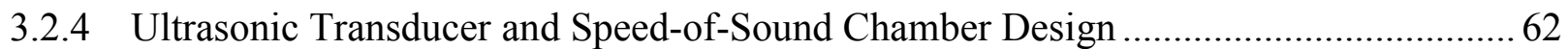

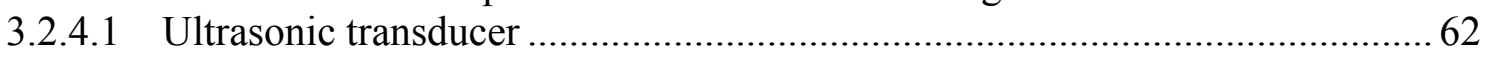

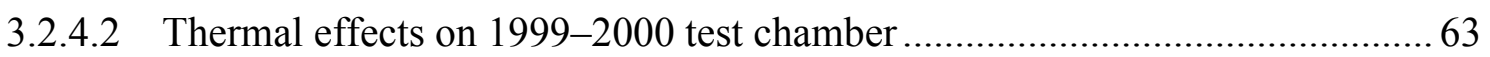

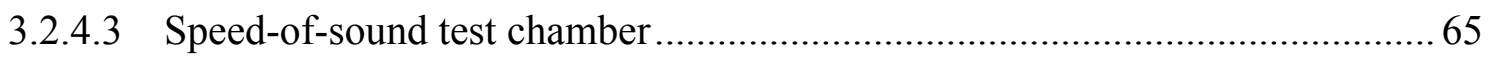

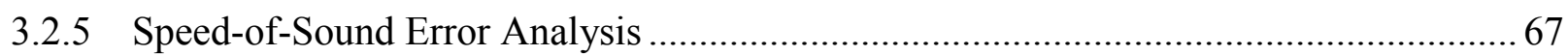

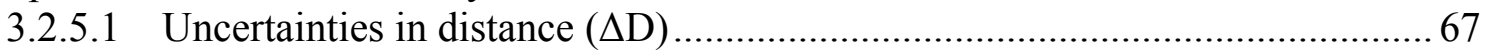

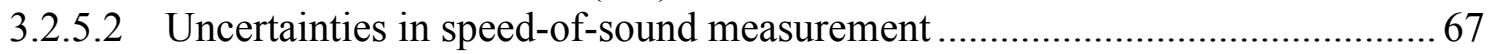

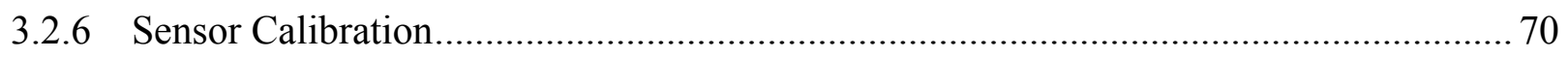

3.2.7 Speed-of-Sound Measurements in Natural Gas ...................................................... 72

3.2.7.1 High-pressure loop tests................................................................... 72

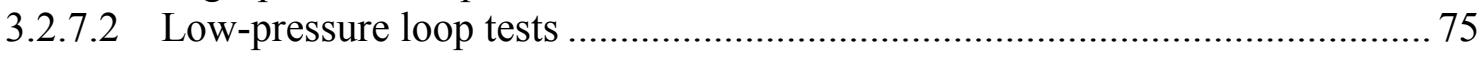

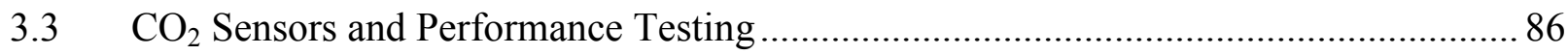

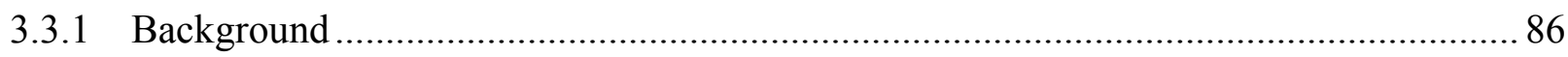

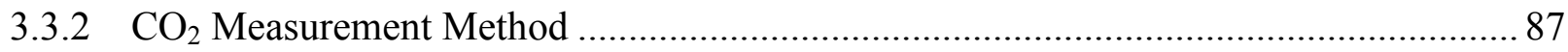

3.3.3 $\mathrm{CO}_{2}$ Sensor Technical Specifications ................................................................ 87

3.3.4 $\mathrm{CO}_{2}$ Sensor Test Considerations and Baseline Test .............................................. 90

3.3.5 $\mathrm{CO}_{2}$ Measurement Chamber for Energy Meter Module .......................................... 91

3.3.6 $\mathrm{CO}_{2}$ Sensor Measurements in Natural Gas ........................................................... 93

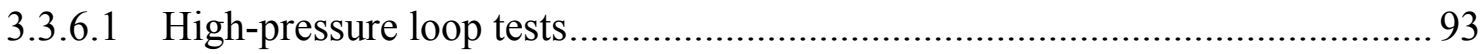

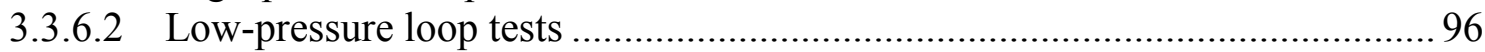

3.4 Evaluation of Inferential Techniques for Sensing $\mathrm{N}_{2}$ Concentration......................... 101

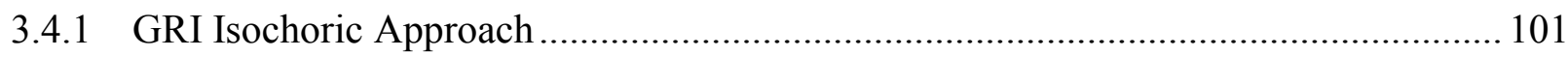

3.4.2 Honeywell Natural Gas Sensor........................................................................... 103

3.4.3 Nuclear Magnetic Resonance ......................................................................... 104

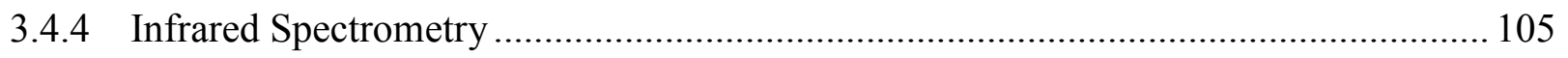

3.4.5 Acoustic Viscosity and Nitrogen Measurements .............................................. 106

4.0 Determination of Gas Heating Value and Energy Flow Rate................................... 107

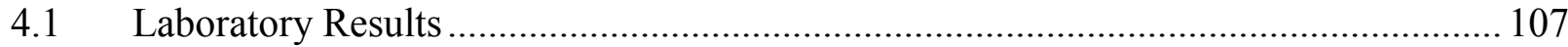

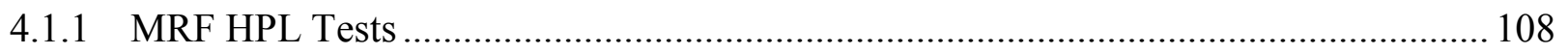

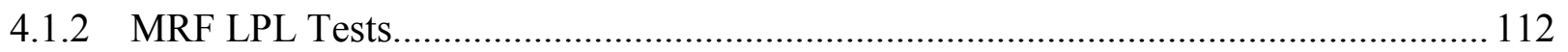

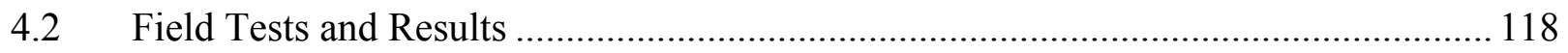




\section{Table of Contents (continued)}

$\begin{array}{lll}\text { Page } & \end{array}$

5.0 Conclusions and Recommendations ...................................................................... 127

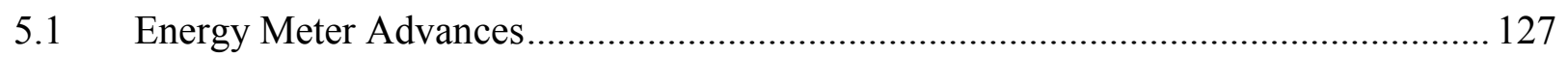

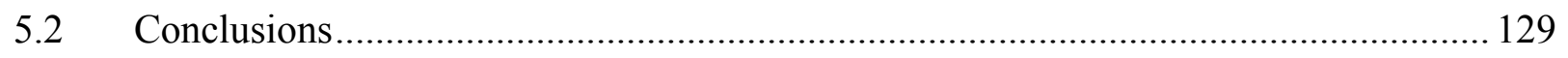

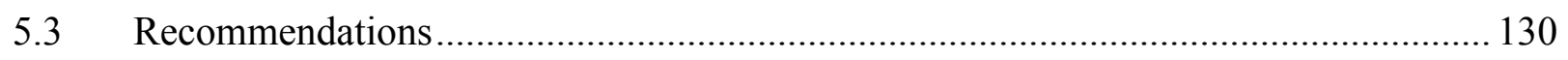

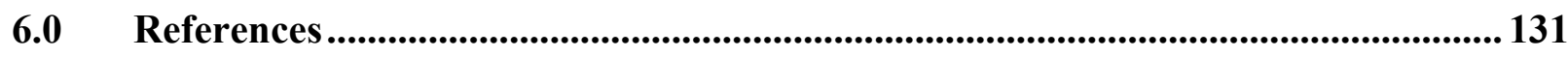

Appendix: Bench Tests of Speed-of-Sound Transducers ...................................................... 135

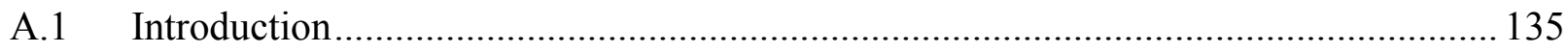

A.2 Experimental Test Parameters and Method .......................................................... 135

A.3 Calculated Speed of Sound in Test Gas Mixtures .................................................. 137

A.4 Bench Test Procedure ........................................................................................ 137

A.5 200-kHz Transducer Tests ................................................................................... 140

A.6 220-kHz Transducer Tests .............................................................................. 153

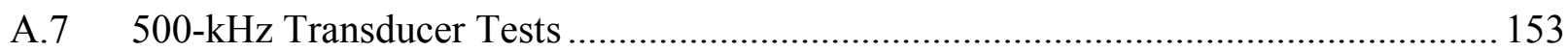

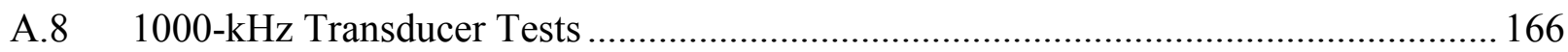

A.9 279-kHz Transducer Tests ............................................................................ 171

A.10 Summary of Bench Test Results..................................................................... 184 
This page is intentionally blank. 


\section{List of Figures}

Figure

Page

2.1 Flow chart of "6 percent algorithm" for gas properties at arbitrary temperatures and pressures ...........................................................................

2.2 Algorithm errors in gas density at sample conditions ....................................... 14

2.3 Algorithm errors in gas density at flowing conditions ....................................... 15

2.4 Algorithm errors in molecular weight ......................................................... 15

2.5 Algorithm errors in mass-based heating value ................................................ 16

2.6 Estimated algorithm errors in gas density at STP ........................................ 17

2.7 Estimated algorithm errors in volumetric heating value ..................................... 18

2.8 Residuals in improved correlation to mass-based heating values of 20 percent database gases within tariff limits .................................................. 28

2.9 Dependence of improved correlation residuals on oxygen content of correlated gases ........................................................................................ 29

2.10 Residuals in improved correlation to mass-based heating values when oxygen is included.

2.11 Residuals in improved correlation to mass-based heating values of 20 percent database gases within AGA-8 normal range limits

2.12 Residuals in improved correlation to densities at STP of 20 percent database gases within AGA-8 normal range limits ........................................... $\quad 30$

3.1 Schematic drawing of pitch-catch method ..................................................... 35

3.2 Schematic drawing of pulse-echo method ...................................................... 35

3.3 Schematic drawing of two-reflector pulse-echo method .................................. 36

3.4 Ultrasonic beam profile of 1.0-in. diameter transducer at $500 \mathrm{kHz}$. The near-field/far-field boundary occurs approximately $7 \mathrm{in.} \mathrm{from} \mathrm{transducer} \mathrm{........} 39$

3.5 Near-field on-axis diffraction interference for 1-in. diameter ultrasonic transducer

3.6 Axisymmetrical near-field amplitude distribution about transducer axis ........... 43

3.7 Combined output signal amplitude from both reflectors ..................................... 44

3.8 Near-field diffraction phase delay and speed-of-sound errors vs. two-way reflection distance

3.9 Ultrasonic velocity dispersion in Natural Gas \#3 using approximate dispersion parameters in Equation (3.16)

3.10 Ultrasonic attenuation at $1 \mathrm{~atm}$ in Natural Gas \#3 using approximate dispersion and classical attenuation parameters in Equations (3.16) and (3.17)...........

3.11 Ultrasonic attenuation at $3 \mathrm{~atm}$ in Natural Gas \#3 using approximate dispersion and classical attenuation parameters in Equations (3.16) and (3.17)...

3.12 Ultrasonic attenuation at $7 \mathrm{~atm}$ in Natural Gas \#3 using approximate dispersion and classical attenuation parameters in Equations (3.16) and (3.17)... 


\section{List of Figures (continued)}

Figure

Page

3.13 Speed-of-sound measurement and signal processing data flow

3.14 Simulated pulse at $300 \mathrm{kHz}$ having $-6 \mathrm{~dB}$ bandwidth of $13.3 \%$ of center frequency

3.15 Simulated pulse at $300 \mathrm{kHz}$ having $-6 \mathrm{~dB}$ bandwidth of $40 \%$ of center frequency

3.16 Simulated pulse at $300 \mathrm{kHz}$ having $-6 \mathrm{~dB}$ bandwidth of $100 \%$ of center frequency

3.17 Reflected signals from Target 1 and Target 2 in 1999-2000 speed-of-sound test chamber

3.18 Cross-correlation function for reflections from Target 1 and Target 2 in 1999-2000 speed-of-sound test chamber

3.19 Speed of sound vs. pressure: Etalon CIA-3525-SB 279-kHz ultrasonic transducer in Natural Gas \#3

3.20 Pulse signals, frequency spectra, and cross-correlation function:

Etalon CIA-3525-SB $279 \mathrm{kHz}$ ultrasonic transducer in Natural Gas \#3.

Pressure: $60.26 \mathrm{psia}$; temperature: $73.5^{\circ} \mathrm{F}$

3.21 Schematic drawing of 1999-2000 speed-of-sound test chamber

3.22 Photograph of Etalon Model CIA-3525-SB2 ultrasonic transducer ...

3.23 Photograph of thermal effects test setup

3.24 Layout drawing of speed-of-sound test chamber 66

3.25 Cutaway drawing of speed-of-sound test chamber 66

3.26 Pre-test calibration of speed-of-sound sensor on MRF HPL ………………...... 71

3.27 Post-test calibration of speed-of-sound sensor on MRF HPL

3.28 Pulse signals, frequency spectra, and cross-correlation function:

Etalon CIA-3525-SB2 266-kHz ultrasonic transducer in MRF HPL gas.

Pressure: 99.02 psia; temperature: $82.26^{\circ} \mathrm{F}$

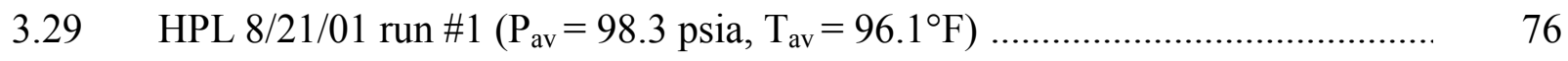

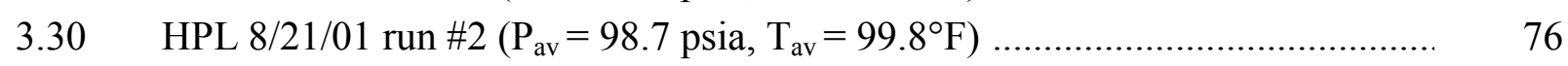

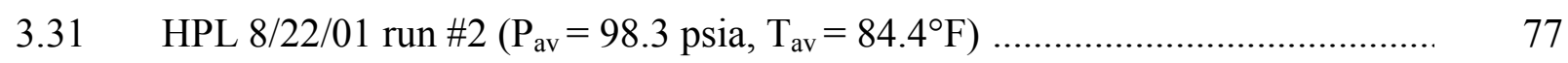

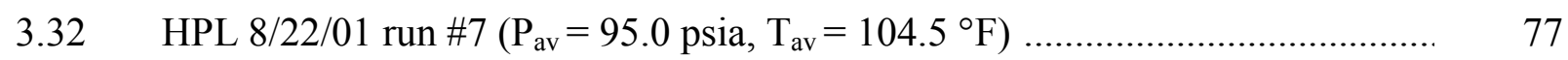

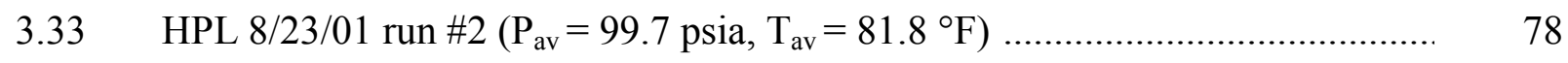

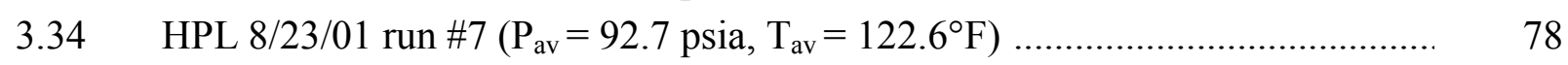

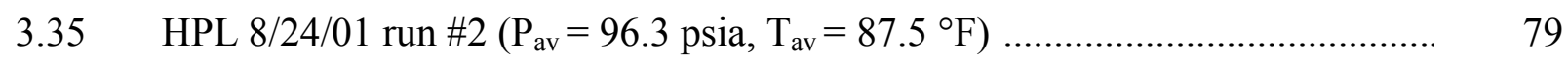

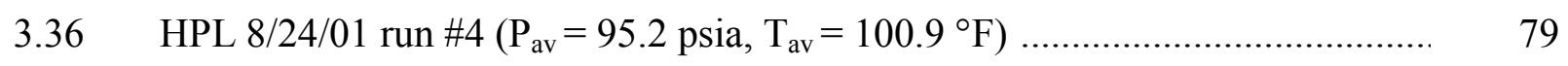

3.37 Pulse signals, frequency spectra, and cross-correlation function:

Etalon CIA-3525-SB2 266-kHz ultrasonic transducer in MRF LPL gas.

Pressure: 107.35 psia; temperature: $96.13^{\circ} \mathrm{F}$. 


\section{List of Figures (continued)}

Figure

Page

3.38 LPL 9/7/01 run \#3 $\left(\mathrm{P}_{\mathrm{av}}=107 \mathrm{psia}, \mathrm{T}_{\mathrm{av}}=84^{\circ} \mathrm{F}, \mathrm{CO}_{2 \mathrm{av}}=1.158 \mathrm{~mol} \%\right)$

and run $\# 4\left(\mathrm{P}_{\mathrm{av}}=51 \mathrm{psia}, \mathrm{T}_{\mathrm{av}}=84.1^{\circ} \mathrm{F}, \mathrm{CO}_{2 \mathrm{av}}=1.157 \mathrm{~mol} \%\right)$

3.39 LPL 9/7/01 run \#5 $\left(\mathrm{P}_{\mathrm{av}}=49.1 \mathrm{psia}, \mathrm{T}_{\mathrm{av}}=89.3^{\circ} \mathrm{F}, \mathrm{CO}_{2 \mathrm{av}}=9.379 \mathrm{~mol} \%\right)$ and run $\# 6\left(\mathrm{P}_{\mathrm{av}}=107.5 \mathrm{psia}, \mathrm{T}_{\mathrm{av}}=90.6^{\circ} \mathrm{F}, \mathrm{CO}_{2 \mathrm{av}}=9.281 \mathrm{~mol} \%\right)$

LPL 9/10/01 run \#2 ( $\left.\mathrm{P}_{\mathrm{av}}=109.9 \mathrm{psia}, \mathrm{T}_{\mathrm{av}}=81.1^{\circ} \mathrm{F}, \mathrm{CO}_{2 \mathrm{av}}=3.35 \mathrm{~mol} \%\right)$

LPL 9/10/01 run \#5 $\left(\mathrm{P}_{\mathrm{av}}=50.8 \mathrm{psia}, \mathrm{T}_{\mathrm{av}}=84.9^{\circ} \mathrm{F}, \mathrm{CO}_{2 \mathrm{av}}=3.050 \mathrm{~mol} \%\right)$.

LPL 9/11/01 run \#1 $\left(\mathrm{P}_{\mathrm{av}}=49.9 \mathrm{psia}, \mathrm{T}_{\mathrm{av}}=81.8^{\circ} \mathrm{F}, \mathrm{CO}_{2 \mathrm{av}}=6.063 \mathrm{~mol} \%\right)$.

3.48 Layout drawing of $\mathrm{CO}_{2}$ test chamber

3.50 MRF testing of $\mathrm{CO}_{2}$ sensor on $8 / 21 / 01$ in HPL

3.51 MRF testing of $\mathrm{CO}_{2}$ sensor on $8 / 22 / 01$ in HPL

3.52 MRF testing of $\mathrm{CO}_{2}$ sensor on $8 / 23 / 01$ in HPL

3.53 MRF testing of $\mathrm{CO}_{2}$ sensor on $8 / 24 / 01$ in HPL

3.60 MRF LPL testing of $\mathrm{CO}_{2}$ sensor on 9/11/01 $\left(\mathrm{CO}_{2}\right.$ av $\left.=8.98 \mathrm{~mol} \%\right)$

4.1 HPL Test 1 energy meter heating value error. Line pressure $=800 \mathrm{psig}$, line temperature $=90^{\circ} \mathrm{F}$

4.2 HPL Test 2 energy meter heating value error. Line pressure $=500 \mathrm{psig}$, line temperature $=50^{\circ} \mathrm{F}$

4.3 HPL Test 3 energy meter heating value error. Line pressure $=500 \mathrm{psig}$, line temperature $=90^{\circ} \mathrm{F}$

4.4 HPL Test 4 energy meter heating value error. Line pressure $=800 \mathrm{psig}$, line temperature $=50^{\circ} \mathrm{F}$

4.5 HPL Test 5 energy meter heating value error. Line pressure $=200$ psig, line temperature $=50^{\circ} \mathrm{F}$ 


\section{List of Figures (continued)}

Figure

Page

4.6 HPL Test 6 energy meter heating value error. Line pressure $=200$ psig,

line temperature $=90^{\circ} \mathrm{F}$

4.7 Summary of HPL tests: energy meter average heating value and average energy flow rate error

4.8 LPL Test 1: energy meter heating value error at line $\mathrm{T}=50^{\circ} \mathrm{F}$ and $\mathrm{CO}_{2}=1.1 \%$

4.9 LPL Test 2: energy meter heating value error at line $\mathrm{T}=90^{\circ} \mathrm{F}$ and $\mathrm{CO}_{2}=1.15 \%$

4.10 LPL Test 3: energy meter heating value error at line $\mathrm{T}=50^{\circ} \mathrm{F}$ and $\mathrm{CO}_{2}=3.10 \%$

4.11 LPL Test 4: energy meter heating value error at line $\mathrm{T}=50^{\circ} \mathrm{F}$ and $\mathrm{CO}_{2}=6.40 \%$

4.12 LPL Test 5: energy meter heating value error at line $\mathrm{T}=90^{\circ} \mathrm{F}$ and $\mathrm{CO}_{2}=6.60 \%$

4.13 LPL Test 6: energy meter heating value error at line $\mathrm{T}=90^{\circ} \mathrm{F}$ and $\mathrm{CO}_{2}=9.80 \%$

4.14 Summary of LPL tests: energy meter average heating value and average energy flow rate error

4.15 LPL tests: correlation between $\mathrm{CO}_{2}$ concentration (in $\mathrm{mol} \%$ ) and $\mathrm{H}_{\mathrm{v}}$ error (in Btu/scf)

4.16 Summary of LPL tests: energy meter average heating value and average energy flow rate error

4.17 Configuration of field devices for energy meter algorithm test

4.18 Field site devices: Daniel 2350 Gas Chromatograph (left) and flow computer rack (right) with two Bristol Babcock computers, at top and bottom .

4.19 Energy meter $\mathrm{CO}_{2}$ and $\mathrm{N}_{2}$ values compared to actual concentrations

4.20 Energy meter line temperature compared to USFM2 temperature

4.21 Energy meter line pressure compared to USFM2 pressure

4.22 Comparison of algorithm heating value determination at constant $\mathrm{CO}_{2}$ and $\mathrm{N}_{2}$ concentrations to field site gas chromatograph value

4.23 Adjusted energy meter heating value compared to field site gas chromatograph

4.24 Field site gas chromatograph un-normalized total over two-week period

4.25 Energy meter heating value, compensated for $\mathrm{CO}_{2}$ only, compared to $\mathrm{GC}$ value 


\section{List of Figures (continued)}

Figure

Page

A.1 Calculated speed of sound in five test gases. [All values are scaled to speed of sound in ultra-pure methane at 14.73 psia.]

A.2 Speed of sound measured at $200 \mathrm{kHz}$ in nitrogen

A.3 Example of Siemens DGT 200-kHz ultrasonic waveforms measured in nitrogen and associated cross-correlation function [Test 1].

(a) Pressure: 14.27 psia

(b) Pressure: $40.77 \mathrm{psia}$

(c) Pressure: 70.93 psia

A.4 Speed of sound measured at $200 \mathrm{kHz}$ in methane

A.5 Example of $200-\mathrm{kHz}$ ultrasonic waveforms measured in methane and associated cross-correlation function.

(a) Pressure: 14.19 psia

(b) Pressure: 40.24 psia

(c) Pressure: 70.70 psia

A.6 Speed of sound measured at $200 \mathrm{kHz}$ in Natural Gas \#1

A.7 Example of 200-kHz ultrasonic waveforms measured in Natural Gas \#1 and associated cross-correlation function.

(a) Pressure: 14.44 psia

(b) Pressure: 40.28 psia

(c) Pressure: 69.35 psia

A.8 Speed of sound measured at $500 \mathrm{kHz}$ in nitrogen

A.9 Example of 500-kHz ultrasonic waveforms measured in nitrogen and associated cross-correlation function.
(a) Pressure: 14.44 psia
(b) Pressure: 40.24 psia
(c) Pressure: 69.35 psia

A.10 Speed of sound measured at $500 \mathrm{kHz}$ in Natural Gas \#1

A.11 Example of 500-kHz ultrasonic waveforms measured in Natural Gas \#1 and associated cross-correlation function.

(a) Pressure: 14.38 psia

(b) Pressure: 40.39 psia

(c) Pressure: 69.44 psia

A.12 Speed of sound measured at $500 \mathrm{kHz}$ in Natural Gas \#2 


\section{List of Figures (continued)}

Figure

Page

A.13 Example of 500-kHz ultrasonic waveforms measured in Natural Gas \#2 and associated cross-correlation function.

(a) Pressure: 14.41 psia ...................................................................... 163

(b) Pressure: 29.66 psia ..................................................................... 164

(c) Pressure: 47.54 psia .......................................................................... 165

A.14 Speed of sound measured at $750 \mathrm{kHz}$ in Natural Gas \#1.............................. 167

A.15 Example of 750-kHz ultrasonic waveforms measured in Natural Gas \#1 and associated cross-correlation function.

(a) Pressure: 40.99 psia ...................................................................... 168

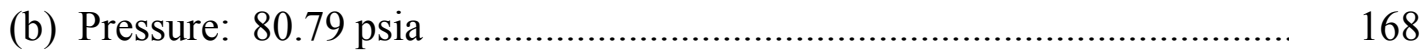

A.16 Speed of sound measured at $750 \mathrm{kHz}$ in Natural Gas \#3 ............................. 169

A.17 Example of 750-kHz ultrasonic waveforms measured in Natural Gas \#3 and associated cross-correlation function.

(a) Pressure: 59.58 psia ........................................................................ 170

(b) Pressure: 99.50 psia ...................................................................... 170

A.18 Speed of sound measured at $279 \mathrm{kHz}$ in nitrogen ..................................... 172

A.19 Example of 279-kHz ultrasonic waveforms measured in nitrogen and associated cross-correlation function.

(a) Pressure: 14.27 psia ........................................................................ 173

(b) Pressure: 40.77 psia ................................................................... 174

(c) Pressure: 70.93 psia ...................................................................... 175

A.20 Speed of sound measured at $279 \mathrm{kHz}$ in Natural Gas \#1 ............................. 176

A.21 Example of 279-kHz ultrasonic waveforms measured in Natural Gas \#1 and associated cross-correlation function.

(a) Pressure: 14.38 psia

(b) Pressure: 40.39 psia ...................................................................... 178

(c) Pressure: 69.44 psia .......................................................................... 179

A.22 Speed of sound measured at $279 \mathrm{kHz}$ in Natural Gas \#3 . .............................. 180

A.23 Example of 279-kHz ultrasonic waveforms measured in Natural Gas \#3 and associated cross-correlation function.

(a) Pressure: 14.41 psia ......................................................................... 181

(b) Pressure: $29.66 \mathrm{psia}$.......................................................................... 182

(c) Pressure: 47.54 psia .......................................................................... 183 


\section{List of Tables}

Table

Page

2.1 Mol\% of gas mixtures within AGA-8 normal range [American Gas Association (1994)]. Amount of constituents not listed is zero...

2.2 Sources of input data and output validation data for tests of 6 percent energy meter algorithm

2.3 Compositions (in mol\%) of test gases used in validating energy meter algorithm [Holste and Hall (1993)]

2.4 Comparison of range of validation data to input limits of 6 percent algorithm

2.5 Gas compositions evaluated in 2001 sensitivity analysis

2.6 Gas conditions evaluated in 2001 sensitivity analysis

2.7 Sensitivities (in terms of standard heating value $\mathrm{H}_{\mathrm{v}}$ ) to input parameters for each gas composition

2.8 Sensitivities (in terms of terms of actual heating value $\rho^{*} \mathrm{H}_{m}$ ) to input parameters for each gas composition

2.9 Uncertainty in standard volumetric heating value $\mathrm{H}_{\mathrm{v}}$

2.10 Uncertainty in actual volumetric heating value $\rho^{*} \mathrm{H}_{\mathrm{m}}$

2.11 Accuracy limits on actual volumetric flow rate imposed by AGA standards

2.12 Comparison of algorithm limits on unmeasured diluents with limits of AGA-8 normal range and typical tariff limits

2.13 Compositions (in mol\%) of gases in 20 percent algorithm database

2.14 Component limits, in binary mixtures with methane, above which observed disagreement between SUPERZ and SonicWare ${ }^{\circledR}$ exceeds $0.005 \%$.

3.1 Speed of sound at three ultrasonic frequencies

3.2 Total attenuation of ultrasonic waves in methane-dominant gas for three frequencies and pressures

3.3 Summary of bench tests on ultrasonic transducers for speedof-sound sensor

3.4 Gas composition used in HPL laboratory tests of energy module

3.5 Gas composition used in LPL laboratory tests of energy module 80

3.6 Carbon dioxide sensor technical specifications

3.7 Comparison of five potential methods for sensing nitrogen content in natural gas 


\section{List of Tables (continued)}

Table

Page

A.1 Calculated speed of sound for five test gases (SonicWare ${ }^{\circledR}$ ) ....................... 138

A.2 Test data at $200 \mathrm{kHz}$ using Siemens DGT transducer ................................ 140

A.3 Test data at $500 \mathrm{kHz}$ using Ultran Model NCT-105 ultrasonic transducer.................................................................................... 153

A.4 Test data at $750 \mathrm{kHz}$ using Ultran Model NCT-510 ultrasonic

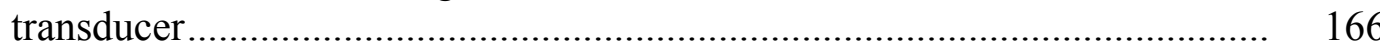

A.5 Test data at $279 \mathrm{kHz}$ using Etalon Model CIA-3525-SB ultrasonic transducer. 


\subsection{Introduction}

\section{$1.1 \quad$ Background}

Natural gas is priced and sold based on the energy delivered to the customer. The value of natural gas depends on the accurate determination of energy flow rate. Historically, the U.S. natural gas industry has determined energy flow rate using independent measurements of flow rate (rate of delivery) and heating value (combustible energy). In order to obtain the heating value of a gas, gas chromatography has traditionally been used to generate a composition assay from samples of the gas. This technology, including installation and operational costs and the sample-taking process required to support gas chromatographs, is often too cost-prohibitive to be used on a wide scale.

Besides the economic considerations, deregulation (FERC Orders 436, 500, 636, etc.) and increased market accessibility have caused supplies to become more varied in composition and less uniform in terms of energy content. Deregulation has expanded the supplier sources from large, single, well-defined sources to include many small suppliers of less defined or well-known compositions. Multiple supply and storage fields with widely varying gas qualities lose their identity when the gases are commingled in the pipeline. Heating value variations from 980 to $1250 \mathrm{Btu} / \mathrm{scf}$ may be recorded at the same locations over a period of time. Because energy content is less predictable, a way to measure energy flow rate locally and less expensively is a crucial need in the natural gas industry.

Energy flow rate measurement is critical to segments of the industry that rely on energy content, whether high or low, as a quality determination factor. Some suppliers, such as those with low-pressure Appalachian gas, provide richer gas than the rest of the country. If energy content is measured correctly, the richer portion can be stripped of its heavier hydrocarbons and sold separately without impacting the quality of the normal pipeline gas, thus providing a product with higher profitability for the suppliers. Most large end-users use gas for fuel and care about energy, because a few-percentage change in heating value can have a large economic impact. Lower heating values mean more gas volume must be purchased, so it is essential that energy rates be measured correctly. Finally, variations greater than $50 \mathrm{Btu} / \mathrm{scf}$ can adversely affect burner efficiencies in furnaces and engines, resulting in reduced operational efficiency.

In 1998, Southwest Research Institute ${ }^{\circledR}\left(\mathrm{SwRI}^{\circledR}\right)$ performed an assessment of natural gas technology and a feasibility evaluation for the U.S. Department of Energy (DOE). The study aimed to compare traditional and alternative technologies for energy flow rate measurement in terms of their accuracy, capital investment, and "operational and maintenance" costs. The existing technology used by the industry is technically sound and fulfills accuracy requirements, but its inherent costs are difficult to justify in most cases. As an alternative to gas chromatographs, SwRI investigated use of an inferential approach to energy measurement developed by Behring et al. [1999]. Behring et al. found that flow and energy measurement properties may be determined with just a few inferential measurements that characterize the natural gas composition without a full composition analysis.

The SwRI study of 1998 determined that this alternative inferential approach to energy measurement was feasible. Heating values and densities may be calculated by sensing the speed 
of sound and the $\mathrm{N}_{2}$ and $\mathrm{CO}_{2}$ concentrations at a known temperature and pressure in a sample and then applying a gross inferential correlation equation. The correlation is based on a database of 102 different natural gas compositions (987-1150 Btu/scf and 83.4-98.3 mol\% methane). This database represents a full practical range of natural gas mixtures under gas quality tariff authority. The inferential approach uses a cubic-spline fit to adjust the pressure and temperature of the reference database to sample conditions and a regression equation to predict molecular weight and heating value (based on the AGA Report No. 8 (AGA-8) Gross Characterization Method [American Gas Association (1994)].

The feasibility of the approach was demonstrated by sensing the speed of sound, the carbon dioxide concentration, the nitrogen concentration, the temperature, and the pressure. Existing ultrasonic transducers and carbon dioxide sensors were used in a proof-of-concept study. Laboratory tests of these sensors in combination with the gas heating value algorithm supported the technical feasibility of the new approach. No sensor for nitrogen concentration was available for this application, but indirect measurement approaches were identified. The 1998 study recommended certain refinements in the speed-of-sound and carbon dioxide sensors and investigation of the suggested indirect nitrogen-sensing methods.

Co-funded by the Gas Research Institute (GRI)* and the U.S. Department of Energy, the project was continued in 1999-2000 with the goal of developing a prototype energy meter module [Morrow et al. (2000)]. The goal of this second phase of work was to retrofit a natural gas custody transfer flow meter for energy measurement at an order-of-magnitude lower cost than a gas chromatograph. This project phase planned to accomplish:

1. a revision of the inferential approach to allow use of the speed of sound at pipeline pressure and temperature as a correlation variable together with the carbon dioxide and nitrogen concentrations;

2. design and construction of a prototype instrument module for energy measurement; and

3. tests of the prototype module at various flowing natural gas conditions in the GRI Metering Research Facility (MRF).

The correlation developed for the inferential technique was revised at the recommendation of the GRI Measurement Technical Advisor Group (MTAG) to use the speed of sound at arbitrary pressure and temperature as a correlation variable. In response to this recommendation, the inferential correlations were reformulated to employ the speed-of-sound and the $\mathrm{N}_{2}$ and $\mathrm{CO}_{2}$ concentrations at arbitrary temperature and pressure without significant loss of accuracy. An energy meter module was then built to measure the inferential parameters $\left(\mathrm{CO}_{2}\right.$, $\mathrm{N}_{2}$, and speed of sound) on a sample gas stream at reduced pressure. The module included specialized sensors for $\mathrm{CO}_{2}$ and speed-of-sound and conventional sensors to measure temperature and pressure. To perform the correlation calculations and apply the AGA-8 Gross Characterization Method, a FORTRAN computer code was translated into ACCOL to run on a Bristol Babcock Model 3330 flow computer commonly used for flow metering installations in the field.

\footnotetext{
* In April 2000, the Gas Research Institute (GRI) and the Institute of Gas Technology (IGT) combined to form the Gas Technology Institute (GTI).
} 
The final project objective of the 1999-2000 work required experimental testing of the prototype module. The energy meter algorithm was tested in combination with an ultrasonic flow meter operating in the MRF High Pressure Loop (HPL). For the ultrasonic flow meter tests, conventional speed-of-sound, pressure, and temperature values at pipeline conditions from the flow meter were substituted for the prototype energy meter module measurements. Carbon dioxide and nitrogen concentrations were obtained from the MRF gas chromatograph. These inputs were fed to the Bristol Babcock flow computer for calculating the standard volumetric gas heating value. Communication problems limited the number of energy meter performance tests within the period of time reserved for testing in the MRF. However, abbreviated tests using a 12in. Daniel Model 3400 ultrasonic meter in the HPL indicated that the standard volumetric heating value was determined accurately to within $\pm 1 \mathrm{Btu} / \mathrm{scf}$.

The prototype energy meter module was tested with a 4-in. orifice flow meter in the MRF Low Pressure Loop (LPL). For these tests, speed of sound and $\mathrm{CO}_{2}$ concentration were measured with the prototype module ultrasonic sensor. These tests showed a $+47 \mathrm{Btu} / \mathrm{scf}$ difference between the measured heating value and the known reference value, due to a variety of factors. However, the measured value of $\mathrm{CO}_{2}$ concentration was within an average of $0.054 \mathrm{~mol} \%$ of the known value determined by gas chromatograph matching, corresponding approximately with the allowable error of $\pm 0.05 \mathrm{~mol} \%$ for this measurement. Because of the large disparity in heating value, project priority was focused on resolving the cause of the heating value error.

Analysis of the experimental test data indicated that the principal source of error was in the measured speed of sound in the gas. Two discrepancies were found in the operating performance of the speed-of-sound sensor, one related to reliably determining the ultrasonic pulse reflection transit time and the second related to measuring the true gas temperature in the speed-of-sound test chamber. When appropriate corrective adjustments were applied to the experimental ultrasonic pulse transit times, the error in heating value was reduced to +15.2 $\mathrm{Btu} / \mathrm{scf}$, demonstrating the strong influence of speed of sound on the derived heating value. Similar adjustments applied to the gas temperature in the speed-of-sound test chamber reduced the heating value error to $2.3 \mathrm{Btu} / \mathrm{scf}$. These results led to positive conclusions regarding the feasibility of the energy meter concept and recommendations for improvements in the ultrasonic transducer and in modifying the speed-of-sound sensor test chamber design so that the gas volume under test was always at the same temperature as the ultrasonic test chamber.

\subsection{Project Tasks and Goals, 2000-2001}

The 1999-2000 energy meter module accuracy target was $\pm 5 \mathrm{Btu} / \mathrm{scf}$, but the experimental results, as briefly summarized above, demonstrated the need for additional refinements to the module in the coming year. Moreover, an accuracy level of $\pm 1-2 \mathrm{Btu} / \mathrm{scf}$ in gas heating value is a desired performance goal for the energy meter if it is to be considered as a replacement for a gas chromatograph. This refined accuracy level was one of the 2001 goals for the energy meter project. To achieve this level of accuracy in an improved prototype energy meter module and to more thoroughly evaluate and expand the inferential algorithm, the 2000-2001 project endeavored to accomplish the following tasks:

1. Assess the inferential algorithm performance when applied to independently determined gas properties listed in the European natural gas database [Holste and Hall (1993)]. Perform sensitivity analysis of the algorithm using several different base gas compositions. 
2. Expand the existing algorithm to allow diluent gas concentrations up to $20 \mathrm{~mol} \%$ in the sample. The expanded algorithm will permit the energy meter to test samples with heating values below $980 \mathrm{Btu} / \mathrm{scf}$.

3. Evaluate an inferential approach to nitrogen sensing either through measurement of thermophysical properties such as specific heat at constant volume and pressure or other nitrogen-dependent parameters.

4. Determine the impact of ambient heat transfer via conduction and radiation between the speed-of-sound test chamber and the gas being tested in the chamber. Modify the test chamber as required to assure that the ultrasonic time-of-flight measurements and gas temperature measurements are accurately consistent.

5. Evaluate commercially available ultrasonic transducers having a higher frequency (in the range of $500 \mathrm{kHz}$ ) and wider operating bandwidth. Perform bench tests on various certified natural gas mixtures to determine the cause of discrepancies between experimental speed-of-sound results, as specifically related to transit time measurements, in comparison with theoretical results computed using SonicWare ${ }^{\circledR}$ software [Lomic, Inc. (1997)].

6. Evaluate the carbon dioxide sensor capabilities over an extended range of $\mathrm{CO}_{2}$ concentration and reduce the sensor uncertainty.

7. Perform additional laboratory tests in the MRF flow loop facilities and perform field tests using the modified and improved energy module. Following successful module tests in the MRF, the GRI MTAG membership will be solicited to assist in arranging the field test of the energy meter module.

\subsection{Summary of Accomplishments, 2000-2001}

Significant progress was made on all of the task objectives planned for 2000-2001. The results are summarized below.

\subsubsection{Tasks 1 and 2: Algorithm Studies}

The original gas heating value algorithm was evaluated using independent gas composition data having constituents within the AGA-8 normal range of composition. The results of the worst-case analysis showed that the individual algorithm parameters (gas density and molecular weight) and volumetric heating value were all within $\pm 0.25 \%$ and, therefore, in close agreement with the original algorithm tolerance. The algorithm was also evaluated to establish the error bounds on input measurements for $\mathrm{CO}_{2}$ and $\mathrm{N}_{2}$ concentrations using a larger database of gases than that used in the 1999-2000 validations. These results tightened the accuracy requirements on speed of sound from $\pm 1.5 \mathrm{ft} / \mathrm{sec}$ to $\pm 0.8 \mathrm{ft} / \mathrm{sec}$ in gases having heating values in the range of 960-1140 Btu/scf with no change in the $\mathrm{CO}_{2}$ and $\mathrm{N}_{2}$ diluent concentrations of $\pm 0.05 \mathrm{~mol} \%$ each. The effects of higher $\mathrm{CO}_{2}$ and $\mathrm{N}_{2}$ concentrations and unmeasured diluents such as might be present in production gas compositions were evaluated using the algorithm in its present (two diluent) form. Based on these results, the present algorithm is not recommended for gases having constituents and compositions outside of the AGA-8 normal range. However, the study showed that with measurements of other diluents, particularly oxygen, the algorithm could potentially be extended to apply to gases having up to $20 \mathrm{~mol} \%$ diluent content. 


\subsubsection{Task 3: Nitrogen Sensing}

Four methods for sensing nitrogen were identified and evaluated for feasibility and practical incorporation as part of the energy meter system. These methods included: (1) inferential measurement of specific heat at constant volume (a patented GRI technique); (2) electronic microsensor methods for measuring either thermal conductivity and specific heats or total absolute viscosity; (3) nuclear magnetic resonance to determine the total hydrogen content of the gas mixture; and (4) infrared spectroscopy to determine the hydrocarbon and $\mathrm{CO}_{2}$ content. Of these methods, the microsensor approach, although requiring additional development, was the most promising method for use in the energy meter application. A fifth method based on acoustic attenuation and dispersion was also identified for future evaluation as a potentially appropriate approach since it could be incorporated as part of the present speed-of-sound sensor.

\subsubsection{Task 4: Thermal Effects Analysis and Design Improvements}

Errors in the speed-of-sound measurements were traced to nonuniform temperature distributions in the speed-of-sound test chamber. Bench tests were performed to isolate the problem factors in the chamber design. An improved speed-of-sound test chamber was designed and used with success in all of the 2000-2001 energy meter tests.

\subsubsection{Task 5: Speed-of-Sound Sensor}

Errors in measuring the travel time of ultrasonic pulses in the speed-of-sound test chamber were caused by the relatively low operating frequency $(200 \mathrm{kHz})$ and narrow bandwidth of the ultrasonic transducer. New commercially available transducers were tested and compared using a wide range of gas compositions and pressures. The most effective transducer (frequency $266 \mathrm{kHz}$; bandwidth $128 \mathrm{kHz}$ ) was adapted to operate in the new speed-of-sound test chamber. The travel time measurement errors caused by correlation cycle skipping were eliminated, resulting in residual errors in speed of sound less than $\pm 1 \mathrm{ft} / \mathrm{sec}$ in the transmission-quality gases tested in the MRF HPL and LPL.

\subsubsection{Task 6: $\mathrm{CO}_{2}$ Sensors}

A commercially available nondispersive infrared absorption sensor was selected for use in the prototype energy meter module. This sensor was thoroughly evaluated under a full range of $\mathrm{CO}_{2}$ concentrations under MRF laboratory flow conditions. This sensor provided $\mathrm{CO}_{2}$ concentrations that were within the $\pm 0.05 \mathrm{~mol} \%$ error bounds required by the heating value algorithm for $\mathrm{CO}_{2}$ concentrations up to $3 \mathrm{~mol} \%$. Measurements at $6 \mathrm{~mol} \%$ and $9 \mathrm{~mol} \%$ revealed nonlinearities in the sensor response, indicating that a more comprehensive calibration method is required for the energy meter application. However, when the nonlinear bias effects were taken into account, this sensing technique was capable of providing $\mathrm{CO}_{2}$ measurements within the accuracy limit required for use in the heating value algorithm.

\subsubsection{Task 7: Laboratory and Field Tests}

The energy meter module was tested on the MRF HPL over a four-day period using a single sample gas composition. The speed-of-sound and $\mathrm{CO}_{2}$ sensors performed with sufficient accuracy to yield gas heating values that were well within the worst-case algorithm error bounds of $\pm 2.4 \mathrm{Btu} / \mathrm{scf}$. When these measurements were translated to energy flow rate, the gas energy 
flow rate errors were less than $2 \%$ when using ultrasonic flow meter accuracies. Similar laboratory tests performed on the MRF LPL over a four-day period utilized normal natural gas samples doped with increasing amounts of dry $\mathrm{CO}_{2}$. Tests on the normal gas $\left(\mathrm{CO}_{2}\right.$ approximately $1.2 \mathrm{~mol} \%$ ) confirmed the energy meter performance observed in the HPL tests. Tests on a gas having an increased $\mathrm{CO}_{2}$ concentration of $3 \mathrm{~mol} \%$ also confirmed the energy meter performance accuracy. Tests at $\mathrm{CO}_{2}$ concentrations of $6 \mathrm{~mol} \%$ and $9 \mathrm{~mol} \%$ revealed significant bias shift and nonlinear response in the $\mathrm{CO}_{2}$ measurements, and a noticeable amount of added scatter in the derived gas heating values. Although the bias effects caused unacceptable offsets in the resulting heating values for gas containing $\mathrm{CO}_{2}$ concentrations above $3 \mathrm{~mol} \%$, when the $\mathrm{CO}_{2}$ sensor bias was removed the residual errors in derived heating value were less than $\pm 2.4 \mathrm{Btu} / \mathrm{scf}$.

A two-week field test was performed at a gas-fired power plant using data acquisition instrumentation to permit the inferential algorithm to be applied as a stand-alone means of determining the gas heating value and to acquire plant operating data adaptable to deriving the gas heating value by approximate simulation of the operating functions of the prototype energy meter module. Practical factors encountered in the data collection process included the fact that update timing in the local flow computers and the time sampling of inputs to the algorithm data logger could not be synchronized and normal operation of the power plant introduced diurnal changes in gas demand. These effects introduced transients in the recorded data and associated inferential gas energy determinations. The transient effects were minimized by analyzing only data recorded after the disturbances died away. The gas heating value derived in the first evaluation scenario (constant $\mathrm{CO}_{2}$ and $\mathrm{N}_{2}$ concentrations) was within $\pm 10 \mathrm{Btu} / \mathrm{scf}$ of the value derived by local gas chromatograph analysis. When the field data were processed again using the monitored values of $\mathrm{CO}_{2}$ concentration, more closely representing the operation of the energy meter module, the agreement with the gas chromatograph value was within the range of -5 to +8 $\mathrm{Btu} / \mathrm{scf}$. 


\subsection{Evaluation and Improvement of Inferential Algorithm}

A major objective of the 1999-2000 energy meter project was to expand the correlation approach developed by Behring et al. [1999]. In this work, reported by Morrow et al. [2000], the original algorithm was extended to provide the option of using the speed of sound from an ultrasonic flow meter in place of speed-of-sound measurements from the retrofit module, and the algorithm was extended to accept measurements of, and compute properties for, arbitrary pressures and temperatures. The original algorithm correlated properties of 102 gases in the GERG (European Gas Research Group) database [Starling et al. (1991)] at standard temperature and pressure (STP) ${ }^{*}$ only. In the revised version, new correlations were based on only nine representative gas mixtures. By using fewer reference gases, it became practical to include gas data at more pressures and temperatures in the correlations, and thus to infer gas density, heating values and energy flow rates from meter data at arbitrary line conditions. Results of the new correlations computed at STP agreed to within $0.02 \%$ with the standard properties of the gases in the GERG database, validating the new correlations.

For the 2000-2001 project year reported here, several tasks were planned to extend and further validate the inferential approach. This chapter presents the results of those tasks. The existing algorithm is reviewed in Section 2.1. The algorithm was validated using experimental data from an independent database of gas compositions; the results are presented in Section 2.2. Section 2.3 presents an investigation of algorithm sensitivities to input variables and an error propagation analysis of the energy meter. Section 2.4 presents the errors due to diluents not measured by the module and therefore not included in the correlations. The potential for an extended algorithm with extended upper limits of nitrogen and carbon dioxide diluents (20 mol\% each) is discussed in Section 2.5. Since the existing algorithm is valid for natural gases with up to $6 \mathrm{~mol} \%$ nitrogen and carbon dioxide, it will be referred to as the " 6 percent algorithm" in the rest of the chapter, to distinguish it from the extended " 20 percent algorithm."

\subsection{Review of Existing Energy Meter Algorithm}

To serve as a frame of reference, this section briefly explains the "6 percent algorithm" for computing gas properties at arbitrary pressures and temperatures. Figure 2.1 is a flow chart of the algorithm. This algorithm has been coded in the FORTRAN language for testing purposes, and in the ACCOL language for field use on a Bristol Babcock 3330 flow computer. The "philosophy" of the algorithm is described here; the reader is referred to Morrow et al. [2000] for details.

The 6 percent algorithm is based on data for nine natural gas mixtures (item a in the first part of Figure 2.1). The compositions of these mixtures fall within the AGA-8 normal range shown in Table 2.1, and the gases span a range of molecular weights and heating values. The sum of nitrogen and carbon dioxide is limited in these mixtures to no more than $6 \mathrm{~mol} \%$. Three of these mixtures consist of only hydrocarbon gases, while the other six contain different amounts of $\mathrm{N}_{2}$ and $\mathrm{CO}_{2}$. The densities and speeds of sound for these nine mixtures

\footnotetext{
${ }^{*}$ In this report, standard temperature and pressure are defined as $60^{\circ} \mathrm{F}$ and 14.73 psia.
} 


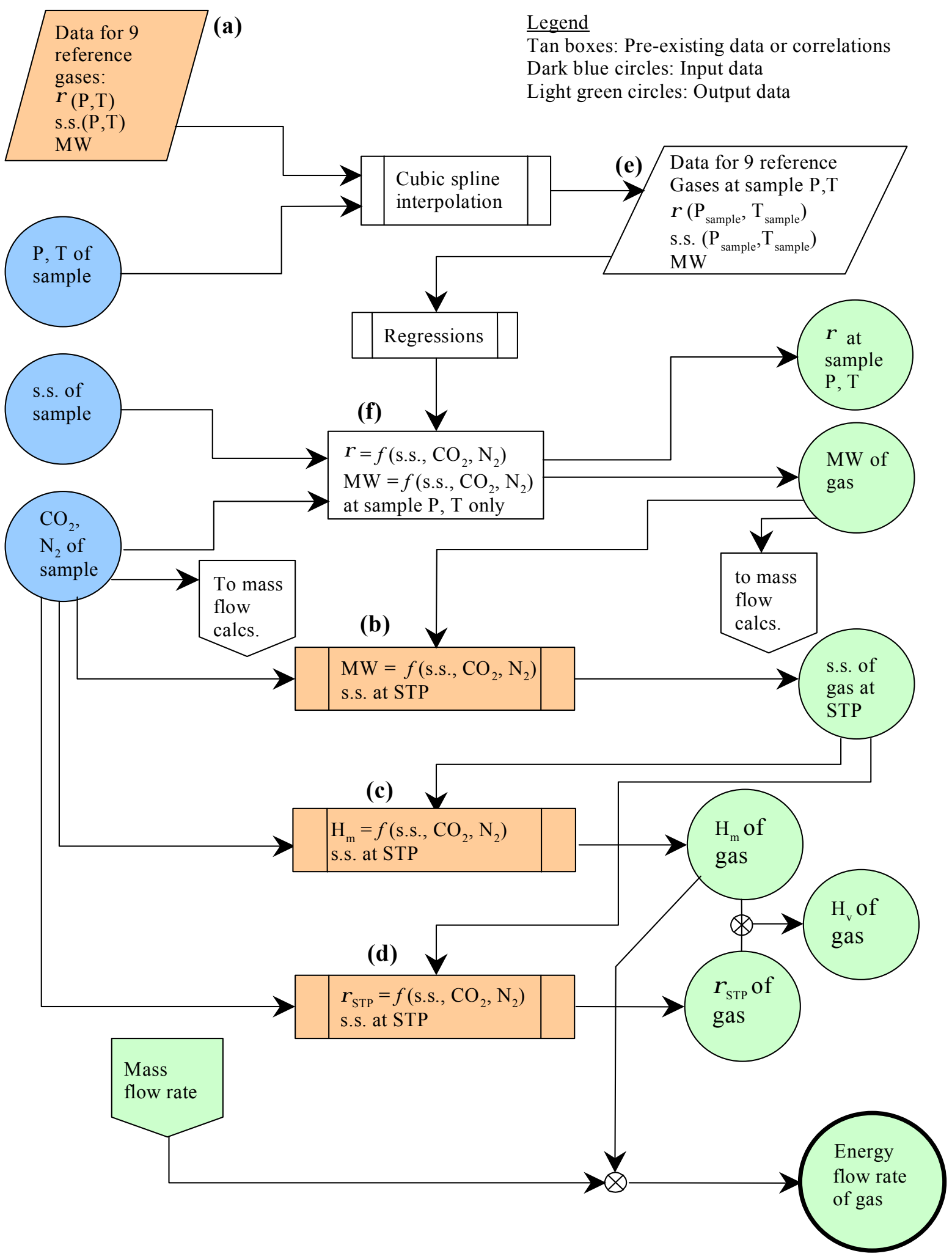

Figure 2.1. Flow chart of "6 percent algorithm" for gas properties at arbitrary temperatures and pressures. 


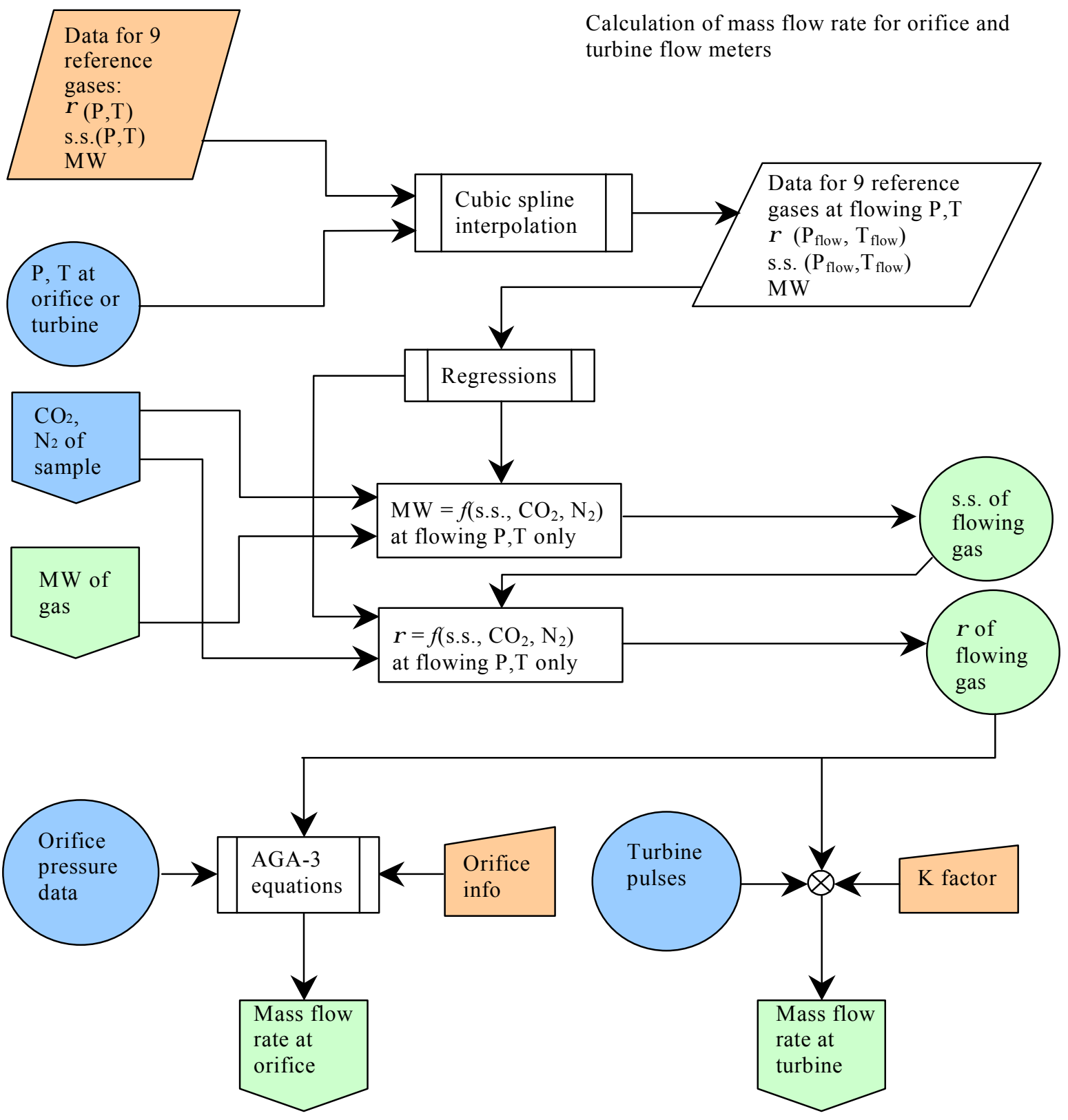

For ultrasonic meters:

sample density = flowing density

mass flow rate $=$ sample density $\mathrm{x}$ volumetric flow rate

Figure 2.1. Flow chart of the "6 percent algorithm" for gas properties At arbitrary temperatures and pressures (continued). 
Table 2.1. Mol\% of gas mixtures within AGA-8 normal range [American Gas Association (1992)]. Amount of constituents not listed is zero.

\begin{tabular}{|l|l|}
\hline Constituent & Mol\% \\
\hline Methane & 45.0 to 100.0 \\
Nitrogen & 0 to 50.0 \\
Carbon Dioxide & 0 to 30.0 \\
Ethane & 0 to 10.0 \\
Propane & 0 to 4.0 \\
Butanes (n-butane + i-butane) & 0 to 1.0 \\
Pentanes (n-pentane + i-pentane) & 0 to 0.3 \\
Hexanes plus & 0 to 0.2 \\
Helium & 0 to 0.2 \\
Water & 0 to 0.05 \\
Hydrogen Sulfide & 0 to 0.02 \\
\hline
\end{tabular}

were computed using SonicWare ${ }^{\circledR}$ [Lomic, Inc. (1997)], while molecular weights were calculated using GPA Standard 2145 [Gas Processors Association (1994)]. Heating values of the mixtures for correlations at STP were also computed using the GPA standard.

Molecular weights, mass-based heating values, and densities at STP are correlated to STP speed-of-sound and diluent concentrations (items $\mathbf{b}$ through $\mathbf{d}$ ), as was done in the original algorithm. These STP correlations do not change with conditions in the pipeline. However, it is also necessary for the 6 percent algorithm to determine heating values using data at different flowing conditions (if speed of sound is measured by an active ultrasonic meter) or sample conditions (if speed of sound is measured in the energy meter module). Thus, densities and sound speeds across a matrix of temperatures and pressures are also included in the nine-mixture database (item a). Properties at arbitrary temperature and pressure can be determined, and then used to find properties at STP using the procedure described next.

When the speed of sound is measured, either by the energy meter module or by an ultrasonic meter, the temperature and pressure at the measurement location are also recorded. Using cubic spline interpolation, properties of the nine database mixtures are found at the measured temperature and pressure (item e), and these properties are fit to correlations (item $\mathbf{f}$ ). Unlike the STP correlations, the correlations of item $\mathbf{f}$ will change as sample or flowing conditions change. These correlations at the sample or flowing state are used together with the measured speed of sound and the diluent concentrations to obtain the density and molecular weight of the gas at the location where speed of sound was measured. Since the molecular weight and diluent mole fractions are independent of the state of the gas, they can be used with the STP correlation for molecular weight (item b) to obtain the speed of sound at STP. Heating values and other desired gas properties are then computed using the STP correlations of items c and d.

By combining the heating value of the gas with a mass flow rate from an active flow meter, the energy flow rate in the pipeline can be calculated. This requires the density of the flowing gas and other information from the flow meter. If an ultrasonic meter is used to obtain speed-of-sound data, the flowing density is already included. If a turbine meter or orifice meter is used with the energy meter module, the density of the flowing gas can be obtained using the temperature and pressure at the flow meter in a procedure similar to items $\mathbf{a}$, e, and $\mathbf{f}$. Appropriate data from the flow meter can then be used to find the mass flow rate and the energy 
flow rate, as shown in the second part of Figure 2.1. In the current ACCOL implementation of the algorithm, the orifice meter and ultrasonic meter options are active, but the turbine meter option has not yet been coded.

\subsection{Validation of Existing Algorithm Using Independent Gas Databases}

The existing 6 percent algorithm for arbitrary pressures and temperatures was developed using values of density and speed of sound predicted by SonicWare ${ }^{\circledR}$. These data, in turn, are based on an extended version of the AGA-8 equation of state [American Gas Association (1992)]. The existing 6 percent algorithm was first validated against natural gas property data from the GERG database [Starling et al. (1991)]. However, this database was also involved in the development of the AGA-8 equations, and some of the gas properties for the validation tests were computed using SonicWare ${ }^{\circledR}$. Since both the algorithm and the initial validation data have origins in the same database, the algorithm was evaluated using an independent database not connected with the AGA-8 equation of state.

The algorithm computes six properties of the natural gas stream (Table 2.2), or if an ultrasonic meter is not used to measure speed of sound, eight properties (since sample conditions at which speed of sound is measured in the module may differ from the line conditions). These six or eight property values must be benchmarked against independently determined values. For a known gas composition, the actual molecular weight and mass-based heating value can be computed directly from properties of the components specified in GPA Standard 2145, and algorithm outputs may be compared to these standard values. Ideally, the other outputs would be compared to independent experimental data: experiment conditions would define the temperature, pressure and diluent content, experimental speed of sound would be input to the algorithm, and the output quantities would be compared to experimentally measured densities and sound speeds or quantities computed from them.

Table 2.2. Sources of input data and output validation data for tests of 6 percent energy meter algorithm.

\begin{tabular}{|c|c|c|c|}
\hline Input to Algorithm & $\begin{array}{l}\text { Source of Input } \\
\text { for Validation Tests }\end{array}$ & $\begin{array}{l}\text { Intermediate or Final } \\
\text { Output of Algorithm }\end{array}$ & $\begin{array}{l}\text { Source of Validation } \\
\text { Data }\end{array}$ \\
\hline $\begin{array}{l}\text { Speed of sound at } \\
\text { sample conditions }\end{array}$ & SonicWare $^{\circledR}$ & $\begin{array}{l}\text { Density at sample } \\
\text { conditions }\end{array}$ & Experimental database \\
\hline $\begin{array}{l}\text { Temperature at sample } \\
\text { conditions }\end{array}$ & Experimental database & Molecular weight & GPA 2145-00 \\
\hline $\begin{array}{l}\text { Pressure at sample } \\
\text { conditions }\end{array}$ & Experimental database & Speed of sound at STP & - \\
\hline $\mathrm{Mol} \% \mathrm{CO}_{2}$ & Experimental database & $\begin{array}{l}\text { Mass-based heating } \\
\text { value, } H_{m}\end{array}$ & GPA $2145-00$ \\
\hline $\mathrm{Mol} \% \mathrm{~N}_{2}$ & Experimental database & Density at STP, $\rho_{\text {STP }}$ & $\begin{array}{l}\text { Extrapolation of } \\
\text { experimental database }\end{array}$ \\
\hline $\begin{array}{l}\text { Temperature at flowing } \\
\text { conditions* }\end{array}$ & Experimental database & $\begin{array}{l}\text { Standard volumetric } \\
\text { heating value, } H_{v}\end{array}$ & $\mathrm{H}_{\mathrm{m}} \times \rho_{\mathrm{STP}}$ \\
\hline \multirow[t]{2}{*}{$\begin{array}{l}\text { Pressure at flowing } \\
\text { conditions }{ }^{*}\end{array}$} & Experimental database & $\begin{array}{l}\text { Speed of sound at } \\
\text { flowing conditions* }\end{array}$ & - \\
\hline & & $\begin{array}{l}\text { Density at flowing } \\
\text { conditions* }\end{array}$ & Experimental database \\
\hline
\end{tabular}


Unfortunately, surveys of the literature [Bruno and Haynes (1993), Diller and Magee (2000)] show that no experimental data are available with both density and speed-of-sound measurements for the same natural gases. Some speed-of-sound measurements are available [Zhang and Schouten (1992), Younglove et al. (1993)] that might be used as input, but no corresponding density data are available to check the resulting output. Therefore, the algorithm was tested using only the available density data, and using SonicWare ${ }^{\circledR}$ to provide the missing speed-of-sound data for the test gases (Table 2.2) since the densities computed by the algorithm are final outputs and should be validated against experimental results. In this case, the speed-ofsound values at STP and at flowing conditions are intermediate outputs of the algorithm. Therefore, since the final outputs that they generate can be validated, the lack of speed-of-sound data to validate these intermediate outputs is not considered critical.

Two independent sets of experimental density data were obtained, one from NEL [Watson (1998)] and the other from a series of round-robin tests sponsored by GRI [Holste and Hall (1993)]. The report by Holste and Hall included experimental data for three different transmission-quality gases, known as RG2, NIST1 and NIST2 (Table 2.3). ${ }^{*}$ These gases meet the diluent limits of the algorithm: up to $6 \% \mathrm{CO}_{2}$, up to $6 \% \mathrm{~N}_{2}$, and all other components within the AGA-8 normal range. Experimental densities for these gases were measured by five different facilities at several combinations of temperature and pressure. Because of the large amount of experimental data in the report, a representative subset of 116 density data points was chosen to test the 6 percent algorithm. The ranges of temperatures, pressures and diluents covered by the experimental data are compared to the limits of the algorithm in Table 2.4. The validation data span most of the temperature and pressure ranges over which the algorithm correlations are applicable, but only include gases at the lower ends of the diluent ranges.

Table 2.3. Compositions (in mol\%) of test gases used in validating energy meter algorithm [Holste and Hall (1993)].

\begin{tabular}{|l|c|c|c|}
\hline Component & RG2 & NIST1 & NIST2 \\
\hline Methane & 85.898 & 96.580 & 90.644 \\
\hline Ethane & 8.499 & 1.815 & 4.553 \\
\hline Propane & 2.296 & 0.405 & 0.833 \\
\hline Isobutane & 0.351 & 0.099 & 0.100 \\
\hline n-Butane & 0.347 & 0.102 & 0.156 \\
\hline Isopentane & 0.051 & 0.047 & 0.030 \\
\hline n-Pentane & 0.053 & 0.032 & 0.045 \\
\hline n-Hexane & 0 & 0.063 & 0.040 \\
\hline Nitrogen & 1.007 & 0.269 & 3.134 \\
\hline Carbon Dioxide & 1.498 & 0.589 & 0.466 \\
\hline Molecular Weight & 18.7708 & 16.7810 & 17.6057 \\
\hline
\end{tabular}

\footnotetext{
* The data were collected at four separate laboratories: NIST (National Institute of Standards and Technology) and Texas A\&M University (TAMU) in the United States, and Ruhrgas and Van der Waals in Europe.
} 
Table 2.4. Comparison of range of validation data to input limits of 6 percent algorithm.

\begin{tabular}{|l|l|l|}
\hline Input variable & Range of validation data & Algorithm limits \\
\hline Temperature & $35.3^{\circ} \mathrm{F}$ to $125.3^{\circ} \mathrm{F}$ & $20^{\circ} \mathrm{F}$ to $150^{\circ} \mathrm{F}$ \\
\hline Pressure & 38 psia to 1400 psia & 14.696 psia to 1400 psia \\
\hline $\mathrm{CO}_{2}$ & $0.466 \mathrm{~mol} \%$ to $1.5 \mathrm{~mol} \%$ & 0 to $6 \mathrm{~mol} \%$ \\
\hline $\mathrm{N}_{2}$ & $0.269 \mathrm{~mol} \%$ to $3.1 \mathrm{~mol} \%$ & 0 to $6 \mathrm{~mol} \%$ \\
\hline
\end{tabular}

The FORTRAN implementation of the 6 percent algorithm was used in the validation tests. The goal of the validation was to accurately reproduce the heating values and measured densities of the test gases from the known test gas compositions and other experimental data. The temperatures and pressures at which the experimental density data were obtained were input as the line temperature and pressure, or as the sample temperature and pressure in the module. The $\mathrm{CO}_{2}$ and $\mathrm{N}_{2}$ contents of the test gases were also input to the algorithm, so that six algorithm inputs in all were taken from the experimental test data. Speed-of-sound data for the test gases were computed using SonicWare ${ }^{\circledR}$. Output densities at sample conditions and flowing conditions were compared directly to experimentally measured densities, since the sample and flowing temperatures and pressures were identical to experimental conditions. Output molecular weights and mass-based heating values were compared to values computed from the experimental composition data and constants in the latest revision to GPA Standard 2145 [Gas Processors Association (2000)]. No experimental density data were available at STP, but STP validation data were extrapolated from an existing dataset using virial equations of state, as will be discussed later.

Figure 2.2 presents algorithm errors in densities at "sample conditions" (the conditions at which speed of sound is measured). These errors are the difference between density computed by the algorithm and experimental density at the same temperature and pressure. Experimental data were reported for the same gas at multiple pressures and temperatures, so densities for the same test gas varied from 0.1 to $6.5 \mathrm{lbm} / \mathrm{ft}^{3}$ among the validation tests. The largest disagreement between the algorithm and experiment is $+0.21 \%$ in sample density. The sample density error is notably largest for gases with measured densities above $4.5 \mathrm{lbm} / \mathrm{ft}^{3}$, but below this level, errors of $\pm 0.1 \%$ are typical. By comparison, uncertainties in the experimental densities are estimated at less than $\pm 0.11 \%$ [Holste and Hall (1993)]. This figure is based on uncertainties in the reported compressibility factors derived from the density data. Below $4.5 \mathrm{lbm} / \mathrm{ft}^{3}$, the densities produced by the algorithm are within the uncertainty limits of the validation data.

Validation of the algorithm results for gas density at flowing conditions is also possible using the same experimental data. Figure 2.3 presents algorithm errors in flowing gas density relative to 70 measured values for the gases in experiments. Additional calculations are required in the algorithm to obtain flowing density and, therefore, more inaccuracy in these values can be expected. The flowing density errors are more widely scattered about zero than the sample density errors, but the maximum observed error in flowing density is within less than $+0.21 \%$ and $-0.1 \%$ in all cases. Again, this compares well with the estimated experimental uncertainties in the density data of $\pm 0.11 \%$ or less.

Figure 2.4 presents errors in algorithm-computed molecular weights relative to values computed from the test gas compositions and GPA 2145-00. While these reference points are not from experiment, they are based on an accepted national standard. For nearly all the validation 
tests, the algorithm computed the molecular weight of the gas to within $\pm 0.02 \%$ of the reference values. By comparison, the estimated uncertainty in the reference values is $\pm 0.13 \%$, based on the uncertainty of the gas composition and the accuracy of the values in GPA 2145. As the density of test gas RG2 increases, a trend of increasing error is evident, but the disagreement between the validation data and the algorithm is still within $\pm 0.10 \%$, and within the uncertainties of the validation data.

Figure 2.5 demonstrates that the algorithm results are also in very good agreement with GPA-based calculations of mass-based heating value. All calculated values agree with values calculated using the GPA standard to within $\pm 0.02 \%$. The validation errors in the figure are all grouped at discrete values, approximately $+0.015 \%,+0.010 \%,-0.010 \%$, and $-0.015 \%$. This is a byproduct of the FORTRAN code, which rounds the heating value to five significant digits as it is written to the output file. In the next section, it will be shown that the uncertainty in heating value due to the accuracy of measured inputs is $\pm 0.24 \%$, one order of magnitude larger than the calculational uncertainty of $\pm 0.02 \%$. While $\pm 0.24 \%$ corresponds to a heating value precision of four significant digits, the calculation of $\mathrm{H}_{\mathrm{v}}$ to five significant figures will prevent rounding and other sources of computational bias from affecting the accuracy of the implemented algorithm.

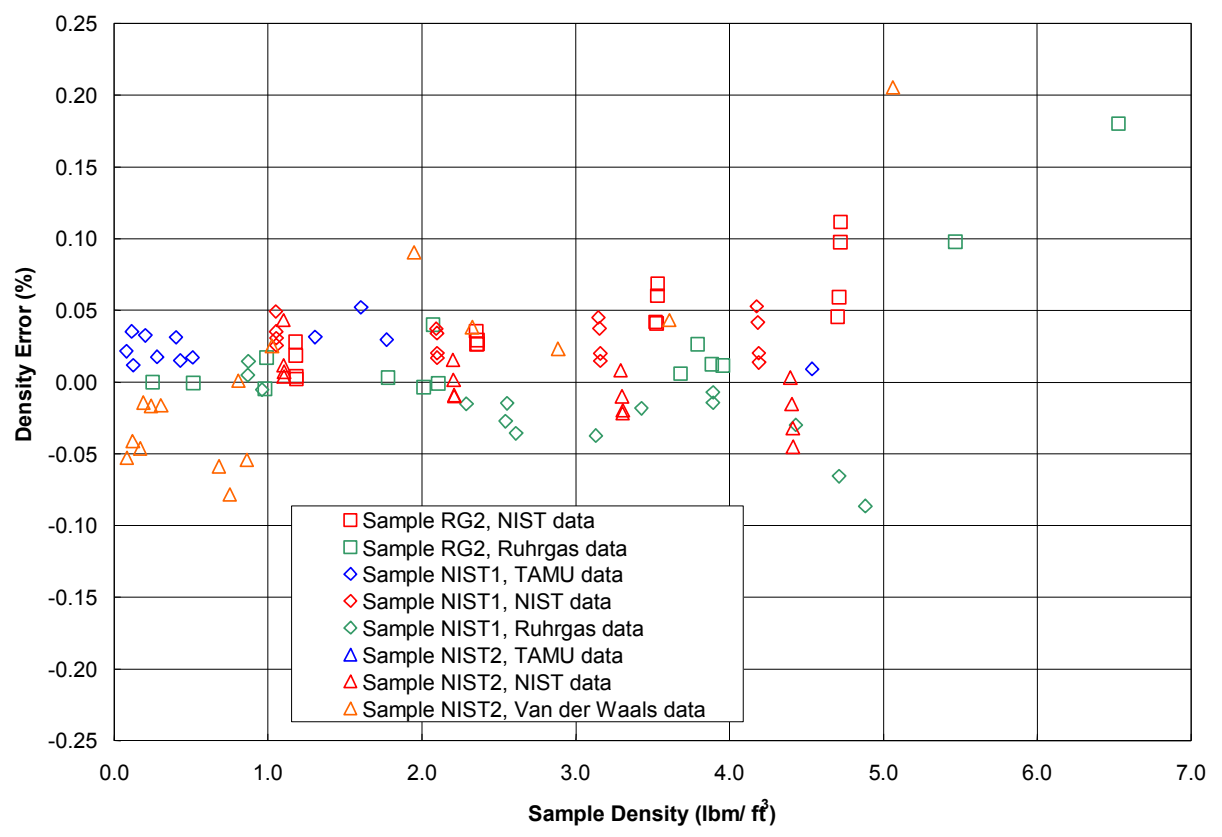

Figure 2.2. Algorithm errors in gas density at sample conditions. 


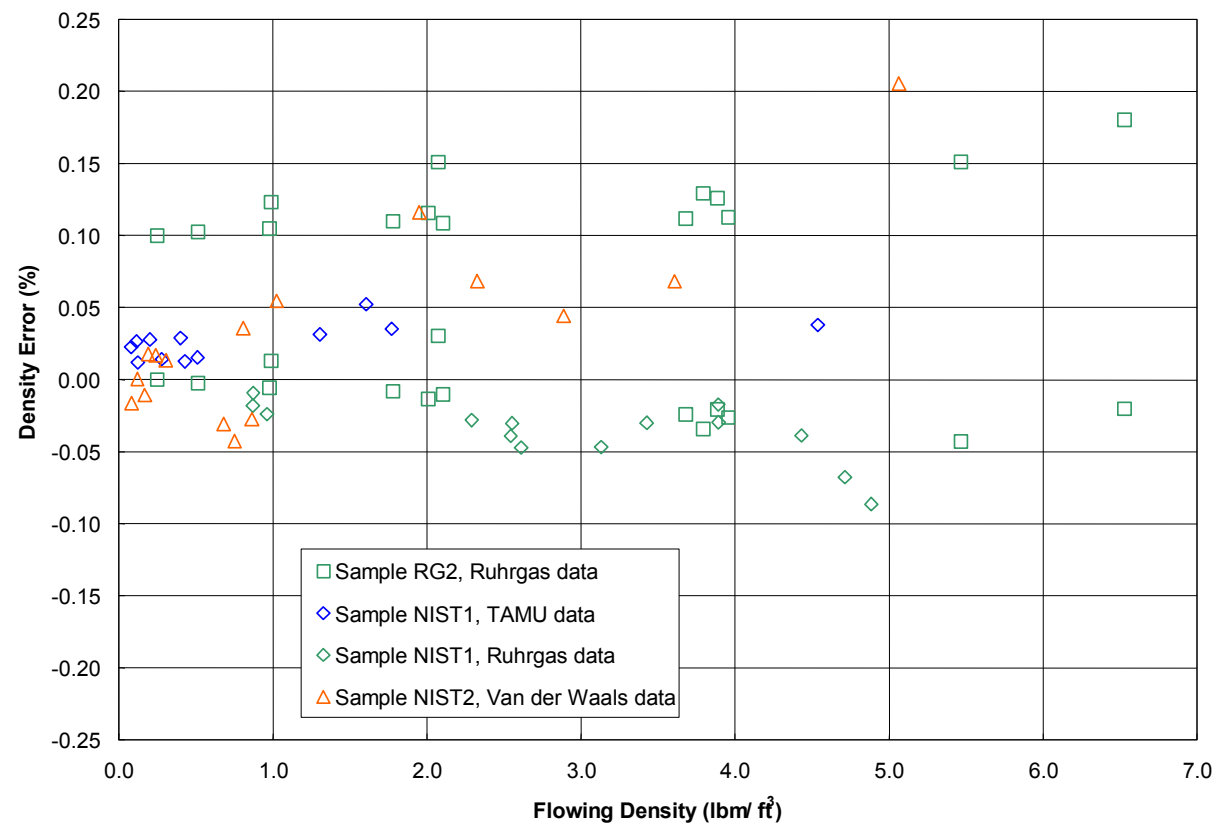

Figure 2.3. Algorithm errors in gas density at flowing conditions.

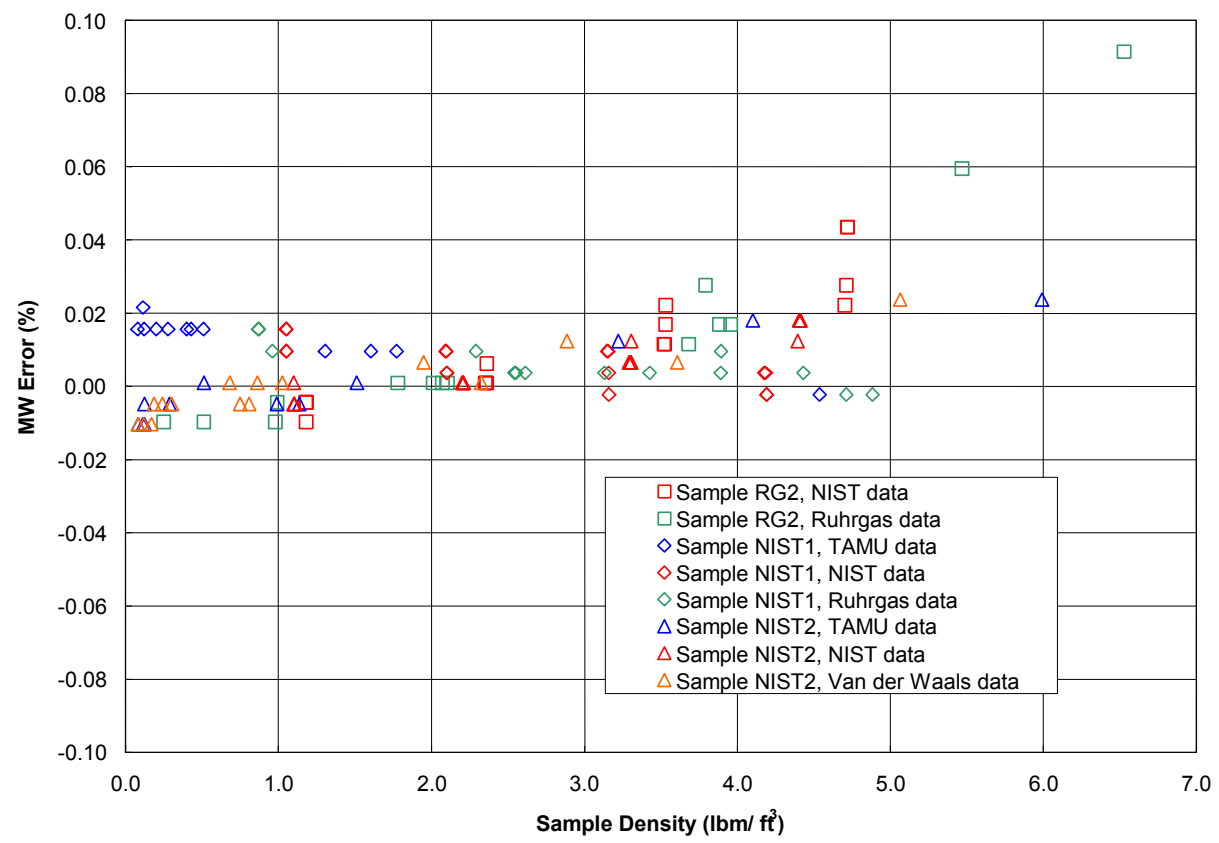

Figure 2.4. Algorithm errors in molecular weight. 


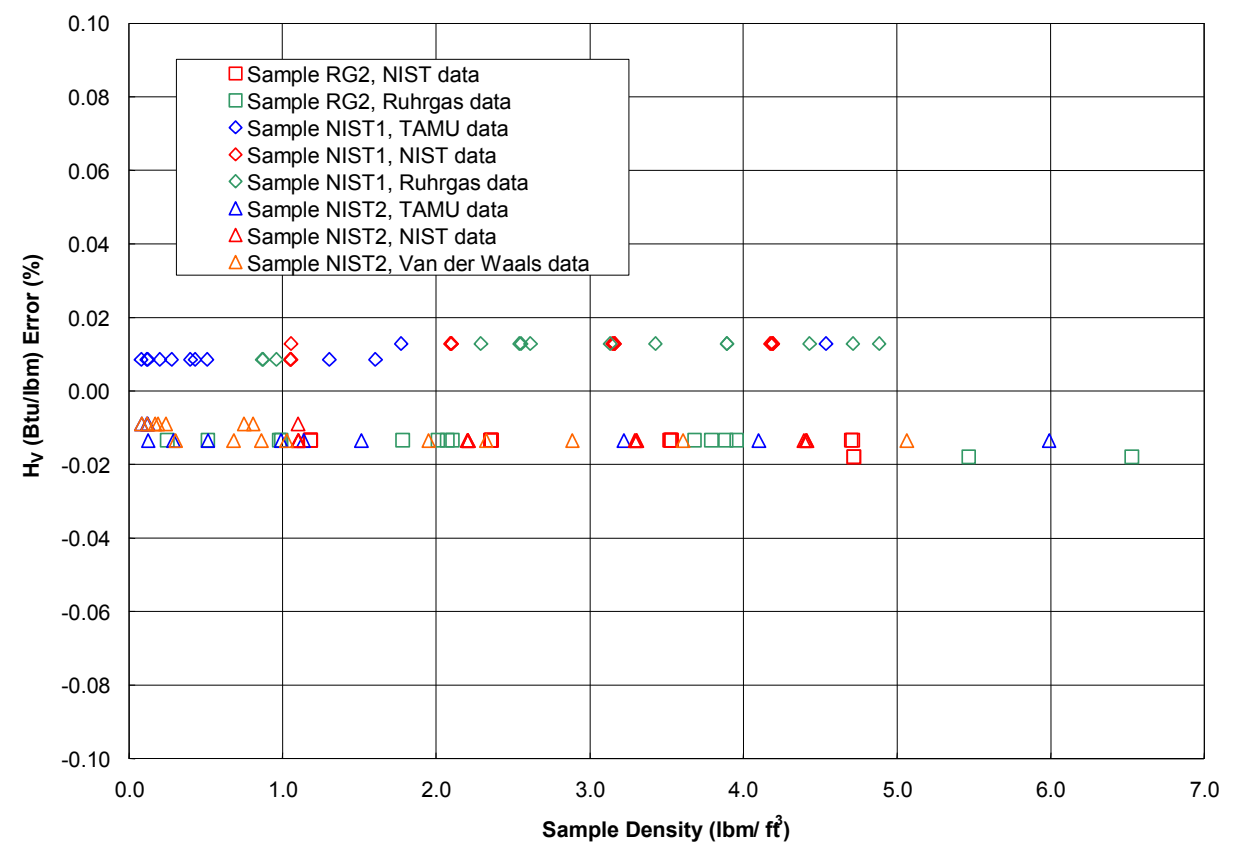

Figure 2.5. Algorithm errors in mass-based heating value.

Because no experimental density data at $60^{\circ} \mathrm{F}$ and 14.73 psia are available, the accuracy of STP gas densities and volumetric heating values from the algorithm must be estimated by other means. For this purpose, a limited set of experimental data was extrapolated to standard pressure using an equation of state. Curve fits of the RG2 data to different equations of state were attempted, including fits to the formulas of van der Waals and Redlich and Kwong [Van Wylen et al. (1994)], but the best fit to the experimental data was produced by a third-order virial equation of state

$$
\frac{P}{\rho R T}=1+\rho B(T)+\rho^{2} C(T)+\rho^{3} D(T)
$$

where the coefficients were of the form

$$
\begin{gathered}
B(T)=b_{0}+b_{1} T+b_{2} T^{2}+b_{3} T^{3} \\
C(T)=c_{0}+c_{1} T+c_{2} T^{2}+c_{3} T^{3} \\
D(T)=d_{0}+d_{1} T+d_{2} T^{2}+d_{3} T^{3}
\end{gathered}
$$


This formula was used to find the density of test gas RG2 at $60^{\circ} \mathrm{F}$ and 14.73 psia for comparison with the algorithm output $\rho_{\mathrm{STP}}$. Because of the range of conditions in the RG2 data used to create the equation of state, this approach indirectly utilized interpolated temperatures and extrapolated pressures to produce the STP data.

Figure 2.6 presents the differences between values of gas density at STP from the algorithm and the extrapolated equation-of-state values. The horizontal axis represents the measured density of the test gas at experimental conditions, which are input to the algorithm as the module energy meter sample conditions. The vertical axis denotes the error in the calculated value of density at STP, assuming the value from the virial equation of state to be correct. The trend of increasing error with increasing density shown here is also seen in the comparisons of sample densities to RG2 data in Figure 2.2. However, the bias in $\rho_{\text {STP }}$ from the algorithm is within $\pm 0.1 \%$ for the RG2 data, a value less than the bias in density at sample conditions for the same test gas.

The mass-based heating value $\mathrm{H}_{\mathrm{m}}$ is multiplied by the density at STP to compute the standard volumetric heating value $\mathrm{H}_{\mathrm{v}}$. This result can be validated using values of $\mathrm{H}_{\mathrm{m}}$ from the GPA standard and the extrapolated density data. In Figure 2.7, algorithm errors in $\mathrm{H}_{\mathrm{v}}$ relative to the benchmark values are plotted for test gas RG2, showing the same trends as in $\rho_{\text {STP. }}$ From these tests, inaccuracy limits of $\pm 0.1 \%$ are observed for the volumetric heating value. Work to extrapolate STP data for the other test gases was not completed due to time constraints, but the Ruhrgas data on RG2 was found to produce the largest disagreement with the algorithm in the other validation tests, so this is considered the "worst-case" comparison at present.

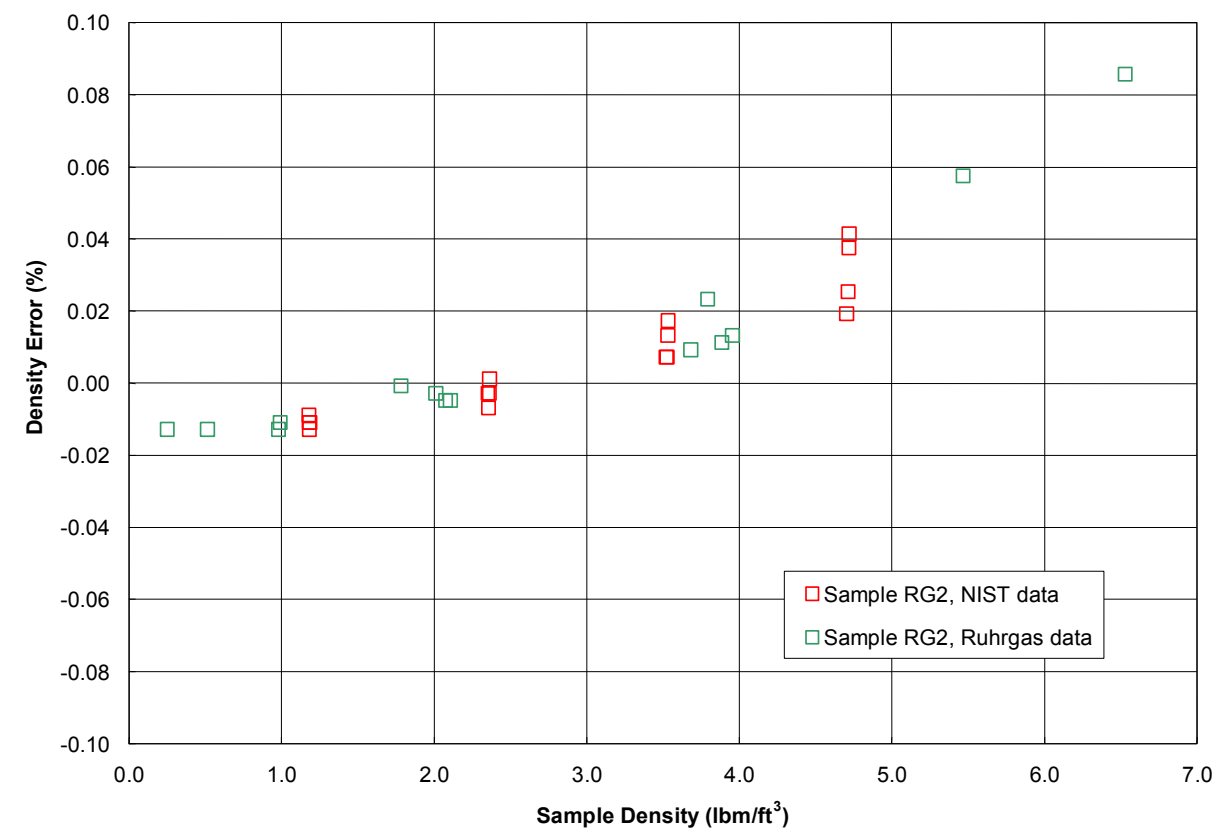

Figure 2.6. Estimated algorithm errors in gas density at STP. 


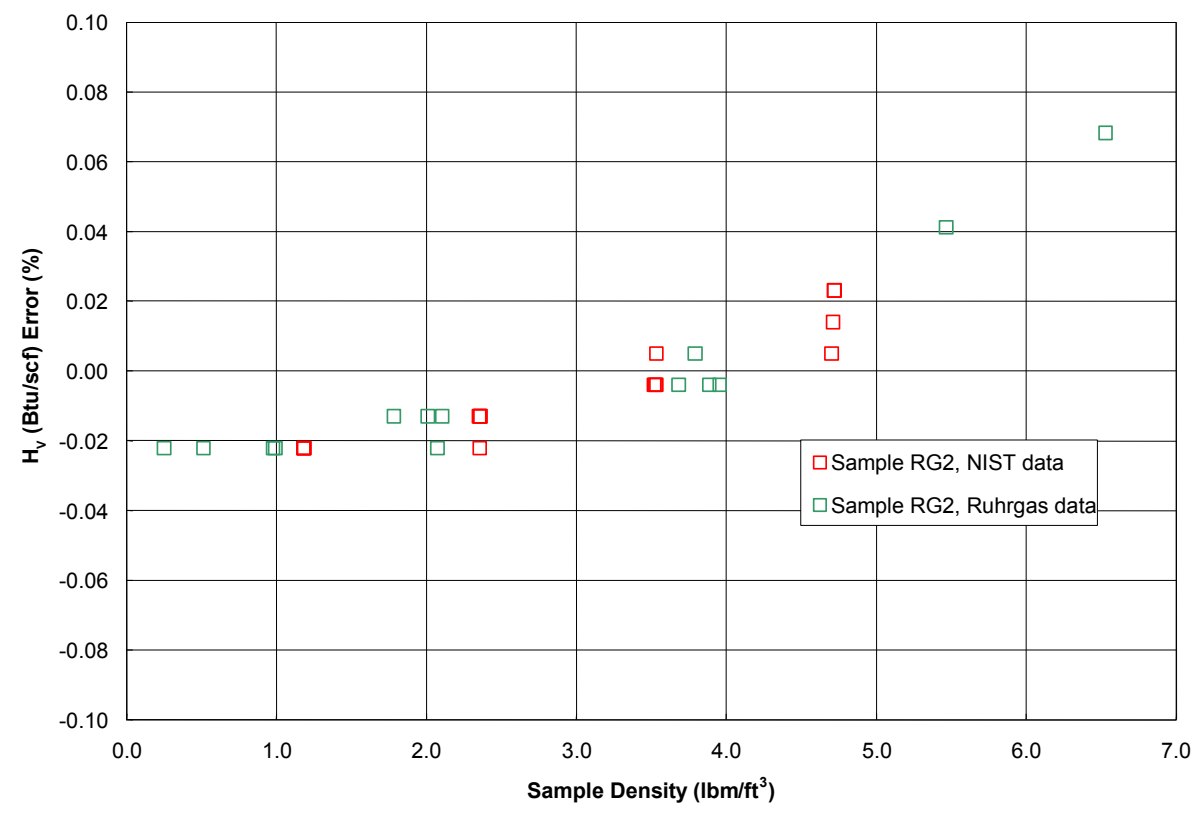

Figure 2.7. Estimated algorithm errors in volumetric heating value.

In summary, the algorithm results are in good agreement with independent experimental validation data. Based on the comparisons performed, the algorithm can compute density to within $\pm 0.21 \%$ in both the energy meter module sample and in pipeline flow. This uncertainty is slightly larger than the estimated uncertainty of $\pm 0.11 \%$ in the validation data. The algorithm can compute gas molecular weights to within $\pm 0.10 \%$, within the estimated uncertainty of the validation data, and can compute mass-based heating values to within $\pm 0.02 \%$. The accuracy limits on density at STP and volumetric heating value from the algorithm are both within $\pm 0.1 \%$.

\subsection{Sensitivity Analyses and Error Propagation}

The uncertainty analysis first reported in 2000 [Morrow et al. (2000)] was used as a starting point to define the measurement uncertainty requirements for each of the inputs to the energy meter algorithm: flowing gas temperature and pressure, speed of sound, $\mathrm{CO}_{2}$ concentration, and $\mathrm{N}_{2}$ concentration. These results have been used to specify the test requirements for the prototype module. However, an uncertainty analysis predicted certain errors in the algorithm computation of standard heating value, $\mathrm{H}_{\mathrm{v}}$, and energy flow rate, $\mathrm{Q}_{\text {energy. }}$ Specifically, the total uncertainty in $\mathrm{H}_{\mathrm{v}}$ resulting from the individual input parameter uncertainties was $\pm 1.85 \mathrm{Btu} / \mathrm{scf}$, assuming that the input parameter uncertainties could be maintained at: $\Delta \mathrm{T}= \pm 0.2^{\circ} \mathrm{F}, \Delta \mathrm{P}= \pm 0.2 \mathrm{psi}, \Delta \mathrm{S}(\mathrm{SOS})= \pm 1.5 \mathrm{ft} / \mathrm{s}, \Delta \mathrm{CO}_{2}= \pm 0.05 \mathrm{~mol} \%, \Delta \mathrm{N}_{2}=$ $\pm 0.05 \mathrm{~mol} \%$. To predict total uncertainty in energy flow rate, Morrow also found that, for $\rho^{*} \mathrm{H}_{\mathrm{m}}$ $=45,183 \mathrm{Btu} / \mathrm{acf}$, the uncertainty in $\rho^{*} \mathrm{H}_{\mathrm{m}}$ would be $\pm 99 \mathrm{Btu} / \mathrm{acf}(+0.22 \%)$ based on the same input uncertainties stated above. The uncertainty in $\mathrm{Q}_{\text {energy }}$ would then be $\pm 0.58 \%$ to $\pm 1.01 \%$, depending on the accuracy of the volumetric flow rate measured by the meter. That analysis gave the 2000-2001 project its present framework for accuracy requirements on each variable to be sampled. 
In order to predict overall uncertainty in the 2000 energy meter report, a typical transmission-quality gas composition was assumed. In the 2001 project, the uncertainty analysis was expanded to include more than one gas composition, since sensitivities of individual input parameters were believed likely to vary as a function of heating value, diluent concentrations, temperature, and pressure. In all, three gas compositions (with different heating values) were evaluated at both high and low temperatures and pressures, as shown in Tables 2.5 and 2.6.

Two measures of heating value were included in the sensitivity analysis, $H_{v}$ and $\rho * H_{m}$, in order to evaluate the algorithm accuracy in determining standard heating value $\mathrm{H}_{\mathrm{v}}$ and energy flow rate. While $\mathrm{H}_{\mathrm{v}}$ is determined only by the algorithm, the energy flow rate is determined by the product of the measured flow rate and the actual volumetric heating value, $\rho * \mathrm{H}_{\mathrm{m}}$, from the algorithm. The algorithm output values for flowing density and mass-based heating value are used to compute actual volumetric energy flow rate and both must therefore be considered in the sensitivity analysis in order to reveal the algorithm accuracy limits for total energy rate.

Table 2.5. Gas compositions evaluated in 2001 sensitivity analysis.

\begin{tabular}{|l|c|c|c|}
\hline $\begin{array}{l}\text { Components } \\
\text { (mol\%) }\end{array}$ & Composition A & Composition B & Composition C \\
\hline Methane & 83.952 & 96.024 & 92.103 \\
\hline Ethane & 9.138 & 1.534 & 1.275 \\
\hline Propane & 3.259 & 0.17633 & 0.05813 \\
\hline i-Butane & 0.5934 & 0.02045 & 0.00552 \\
\hline n-Butane & 0.3956 & 0.03385 & 0.00713 \\
\hline i-Pentane & 0.1254 & 0.01062 & 0.00213 \\
\hline n-Pentane & 0.0836 & 0.00940 & 0.00156 \\
\hline n-Hexane & 0.0473 & 0.00865 & 0.00133 \\
\hline n-Heptane & 0.0139 & 0.00921 & 0.00128 \\
\hline n-Octane & 0.0008 & 0.00263 & 0.00107 \\
\hline n-Nonane & 0.0000 & 0.00110 & 0.00034 \\
\hline Carbon Dioxide & 1.987 & 1.255 & 5.976 \\
\hline Nitrogen & .404 & .9144 & .5659 \\
\hline Total & 100.00 & 100.00 & 100.00 \\
\hline $\mathbf{H}_{\mathbf{v}}$ (Btu/scf) & $\mathbf{1 1 4 1 . 0}$ & $\mathbf{1 0 0 9 . 4}$ & $\mathbf{9 5 9 . 6}$ \\
\hline
\end{tabular}


Table 2.6. Gas conditions evaluated in 2001 sensitivity analysis.

\begin{tabular}{|l|c|c|c|c|}
\hline & $\begin{array}{c}\text { Pressure } \\
\text { (psi) }\end{array}$ & $\begin{array}{c}\text { Temperature } \\
\text { (deg F) }\end{array}$ & $\begin{array}{c}\text { Density } \\
\left(\text { actual, lbm/ft }^{\mathbf{3}} \text { ) }\right.\end{array}$ & $\begin{array}{c}\text { Speed of Sound } \\
\text { (ft/s) }\end{array}$ \\
\hline Composition A Normal & 140 & 70 & 0.4912 & 1294.77 \\
\hline Composition A High Temp & 140 & 90 & 0.4717 & 1318.85 \\
\hline $\begin{array}{l}\text { Composition A High } \\
\text { Pressure }\end{array}$ & 620 & 70 & 2.3939 & 1244.03 \\
\hline $\begin{array}{l}\text { Composition B = Previous } \\
\text { Year Sensitivity Base }\end{array}$ & 620 & 70 & 1.9923 & 1387.46 \\
\hline Composition C Normal & 140 & 70 & 0.4516 & 1369.14 \\
\hline Composition C High Temp & 140 & 90 & 0.434 & 1394.19 \\
\hline $\begin{array}{l}\text { Composition C High } \\
\text { Pressure }\end{array}$ & 620 & 70 & 2.1384 & 1339.12 \\
\hline
\end{tabular}

\subsubsection{Uncertainty in Heating Values}

The heating value sensitivity study used the same procedure as that used in the previous analysis. The algorithm sensitivity was determined by evaluating its response to small fluctuations in each of the five input parameters ( $\mathrm{T}, \mathrm{P}, \mathrm{S}, \mathrm{CO}_{2}$ concentration, $\mathrm{N}_{2}$ concentration) that affect the heating value calculation. Each input parameter was varied by a small amount while the other four inputs were held fixed as the algorithm was run. Then, the sensitivity of the algorithm output (in terms of actual and standard volumetric heating value) to small fluctuations of a single input was determined. The resulting sensitivities for each gas composition were compared, and the highest sensitivity was selected as the "worst case" value. A comparison of results for each composition is shown in Table 2.7 (in terms of $\mathrm{H}_{\mathrm{v}}$ ) and Table 2.8 (in terms of $\rho^{*} \mathrm{H}_{\mathrm{m}}$ ).

The "worst-case" sensitivities were used in combination with accuracy limitations on each of the input parameters to estimate the total uncertainty of standard and actual heating values shown in Tables 2.9 and 2.10. The accuracy limits on pressure and temperature were chosen based on the GRI Metering Research Facility best possible measurement accuracy. Allowable uncertainty limits for $\mathrm{CO}_{2}$ concentration, $\mathrm{N}_{2}$ concentration, and speed-of-sound measurement were based on the 2001 project design goals for the current prototype module sensors. Total uncertainty in the algorithm output was calculated based on the root-mean-square value of the individual uncertainties as follows:

For $\mathrm{H}_{\mathrm{v}}$ :

$$
\Delta H_{v}=\sqrt{\left(\frac{\delta H_{v}}{\delta T} \times \Delta T\right)^{2}+\left(\frac{\delta H_{v}}{\delta P} \times \Delta P\right)^{2}+\left(\frac{\delta H_{v}}{\delta V_{s}} \times \Delta S\right)^{2}+\left(\frac{\delta H_{v}}{\delta X_{C O 2}} \times \Delta X_{C O 2}\right)^{2}+\left(\frac{\delta H_{v}}{\delta X_{N 2}} \times \Delta X_{N 2}\right)^{2}}
$$


For $\rho^{*} \mathrm{H}_{\mathrm{m}}$

$\Delta\left(\rho H_{m}\right)=\sqrt{\left(\frac{\delta \rho H_{m}}{\delta T} \times \Delta T\right)^{2}+\left(\frac{\delta \rho H_{m}}{\delta P} \times \Delta P\right)^{2}+\left(\frac{\delta \rho H_{m}}{\delta V_{s}} \times \Delta S\right)^{2}+\left(\frac{\delta \rho H_{m}}{\delta X_{C O 2}} \times \Delta X_{C O 2}\right)^{2}+\left(\frac{\delta \rho H_{m}}{\delta X_{N 2}} \times \Delta X_{N 2}\right)^{2}}$

The total uncertainty in standard volumetric heating value is $\pm 2.4 \mathrm{Btu} / \mathrm{scf}$, approximately $\pm 0.24 \%$ if $\mathrm{H}_{\mathrm{v}}=1009 \mathrm{Btu} / \mathrm{scf}$. This estimate of uncertainty is higher than the estimate of \pm 1.85 $\mathrm{Btu} / \mathrm{scf}$ determined by the single transmission-quality gas sensitivity analysis in 1999-2000. However, this new estimate includes the "worst case," where sensitivities are higher than those expected for more typical natural gas mixtures. It is evident from Tables 2.7 and 2.8 that the sensitivity coefficients are a function of gas composition. If the gas being measured is similar to the MRF distribution-quality gas used in the 1999-2000 analysis (Composition B in the tables), the coefficients will be smaller. The allowable measurement uncertainties in Table 2.9 will then lead to a smaller total uncertainty in $\mathrm{H}_{\mathrm{v}}$. The "worst case" sensitivity coefficients in the present analysis are all derived from Composition A, which includes higher amounts of ethane and heavier hydrocarbons than are found in typical transmission and distribution gases. The total uncertainty of $\pm 2.4 \mathrm{Btu} / \mathrm{scf}$ in heating value will be referenced in the remainder of this report, but the reader should keep in mind that heating value uncertainties in typical transmission-quality natural gas will be smaller than this "worst-case" value.

Input parameters making the largest contribution to the standard heating value uncertainty are the speed-of-sound, $\mathrm{CO}_{2}$ and $\mathrm{N}_{2}$ concentrations, in agreement with the previous sensitivity analysis. Comparatively, the total uncertainty in actual volumetric heating value was on the order of 125 Btu/acf, i.e., approximately $0.27 \%$ if $\rho^{*} \mathrm{H}_{\mathrm{m}}=45,000 \mathrm{Btu} / \mathrm{acf}$, with speed-of-sound, $\mathrm{CO}_{2}$, and $\mathrm{N}_{2}$ concentrations again making the largest contributions to total uncertainty.

Table 2.7. Sensitivities (in terms of standard heating value $H_{v}$ ) to input parameters for each gas composition.

\begin{tabular}{|c|c|c|c|c|c|}
\hline Base Condition & $\begin{array}{c}\delta \mathbf{H}_{\mathrm{v}} / \delta \mathbf{T} \\
(\mathrm{Btu} / \mathrm{scf}) /{ }^{\circ} \mathrm{F}\end{array}$ & $\begin{array}{c}\delta \mathbf{H}_{\mathrm{v}} / \delta \mathbf{P} \\
(\mathrm{Btu} / \mathrm{scf}) / \mathrm{psi}\end{array}$ & $\begin{array}{c}\delta \mathbf{H}_{\mathbf{v}} / \delta \mathbf{S} \\
(\mathrm{Btu} / \mathrm{scf}) /(\mathrm{ft} / \mathrm{s})\end{array}$ & $\begin{array}{c}\delta \mathbf{H}_{\mathrm{v}} / \delta \mathbf{X}_{\mathbf{N 2}} \\
(\mathrm{Btu} / \mathrm{scf}) / \mathrm{mol} \%\end{array}$ & $\begin{array}{c}\delta \mathbf{H}_{\mathrm{v}} / \delta \mathbf{X}_{\mathbf{C O} 2} \\
(\mathrm{Btu} / \mathrm{scf}) / \mathrm{mol} \%\end{array}$ \\
\hline A Normal & 1.58 & -0.16 & -1.3 & -14.71 & -22.71 \\
\hline A High Temp & 1.5 & -0.14 & -1.27 & -14.79 & -22.43 \\
\hline A High Pressure & 1.75 & -0.09 & -1.05 & -12.82 & -20.64 \\
\hline B Previous Year & 1.45 & -0.03 & -0.9 & -13.2 & -21.18 \\
\hline C Normal & 1.43 & -0.09 & -1.12 & -14.47 & -22.26 \\
\hline C High Temp & 1.36 & -0.07 & -1.11 & -14.51 & -22.32 \\
\hline C High Pressure & 1.53 & -0.04 & -0.96 & -12.93 & -20.8 \\
\hline
\end{tabular}


Table 2.8. Sensitivities (in terms of actual heating value $\rho^{*} H_{m}$ ) to input parameters for each gas composition.

\begin{tabular}{|l|c|c|c|c|c|}
\hline Base Condition & $\begin{array}{c}\delta \rho * \mathbf{H}_{\mathrm{m}} / \delta \mathbf{T} \\
(\mathrm{Btu} / \mathrm{acf}) /{ }^{\circ} \mathrm{F}\end{array}$ & $\begin{array}{c}\delta \rho * \mathbf{H}_{\mathrm{m}} / \delta \mathbf{P} \\
(\mathrm{Btu} / \mathrm{acf}) / \mathrm{psi}\end{array}$ & $\begin{array}{c}\delta \rho * \mathbf{H}_{\mathrm{m}} / \delta \mathbf{S} \\
(\mathrm{Btu} / \mathrm{acf}) /(\mathrm{ft} / \mathrm{s})\end{array}$ & $\begin{array}{c}\delta \rho * \mathbf{H}_{\mathrm{m}} / \delta \mathbf{X}_{\mathrm{N} 2} \\
(\mathrm{Btu} / \mathrm{acf}) / \mathrm{mol} \%\end{array}$ & $\begin{array}{c}\delta \rho * \mathbf{H}_{\mathrm{m}} / \delta \mathbf{X}_{\mathrm{CO} 2} \\
(\mathrm{Btu} / \mathrm{acf}) / \mathrm{mol} \%\end{array}$ \\
\hline A Normal & -6.4 & 78.3 & -13.1 & -146.1 & -221.5 \\
\hline A High Temp & -6.0 & 75.2 & -12.3 & -140.1 & -211.7 \\
\hline A High Pressure & -40.0 & 91.0 & -67.1 & -710.4 & -1088.4 \\
\hline B Previous Year & -32.4 & 76.9 & -50.3 & -684.1 & -1037.3 \\
\hline C Normal & -4.2 & 65.4 & -11.2 & -141.3 & -215.5 \\
\hline C High Temp & -4.0 & 62.8 & -10.5 & -136.1 & -207.2 \\
\hline C High Pressure & -24.4 & 73.2 & -53.8 & -665.8 & -1016.2 \\
\hline \multicolumn{7}{|l|}{ “Worst-Case" Value (i.e., highest sensitivity) is highlighted in bold italics. } \\
\hline
\end{tabular}

Table 2.9. Uncertainty in standard volumetric heating value $\mathbf{H}_{\mathbf{v}}$

\begin{tabular}{|c|c|c|c|}
\hline Uncertainty source & $\begin{array}{l}\text { Allowable } \\
\text { uncertainty }\end{array}$ & $\begin{array}{c}\text { "Worst Case" Sensitivity } \\
\delta \mathrm{H}_{\mathrm{v}} / \delta \text { (input) }\end{array}$ & $\begin{array}{c}U_{95 \%} \\
\text { (Btu/scf) }\end{array}$ \\
\hline Temperature ( $\left.{ }^{\circ} \mathrm{F}\right)$ & \pm 0.2 & 1.75 & \pm 0.350 \\
\hline Pressure (psi) & \pm 0.2 & -0.16 & \pm 0.032 \\
\hline Speed of Sound (ft/s) & \pm 1.5 & -1.30 & \pm 1.950 \\
\hline $\mathrm{N}_{2}$ concentration $(\mathrm{mol} \%)$ & \pm 0.05 & -14.79 & \pm 0.739 \\
\hline \multirow[t]{2}{*}{$\mathrm{CO}_{2}$ concentration (mol\%) } & \pm 0.05 & -22.71 & \pm 1.135 \\
\hline & & Total Uncertainty $\left(\Delta \mathbf{H}_{\mathrm{v}}\right)=$ & \pm 2.40 \\
\hline
\end{tabular}

Table 2.10. Uncertainty in actual volumetric heating value $\rho^{*} \mathbf{H}_{m}$.

\begin{tabular}{|c|c|c|c|}
\hline Uncertainty source & $\begin{array}{l}\text { Allowable } \\
\text { uncertainty }\end{array}$ & $\begin{array}{c}\text { "Worst Case" Sensitivity } \\
\delta \rho^{*} \mathbf{H}_{\mathrm{m}} / \delta \text { (input) }\end{array}$ & $\begin{array}{c}U_{95 \%} \\
\text { (Btu/acf) }\end{array}$ \\
\hline Temperature $\left({ }^{\circ} \mathrm{F}\right)$ & \pm 0.2 & -40.0 & \pm 8.00 \\
\hline Pressure (psi) & \pm 0.2 & 91.0 & \pm 18.20 \\
\hline Speed of Sound (ft/s) & \pm 1.5 & -67.1 & \pm 100.65 \\
\hline $\mathrm{N}_{2}$ concentration $(\mathrm{mol} \%)$ & \pm 0.05 & -710.4 & \pm 35.52 \\
\hline \multirow[t]{2}{*}{$\mathrm{CO}_{2}$ concentration $(\mathrm{mol} \%)$} & \pm 0.05 & -1088.4 & \pm 54.42 \\
\hline & & Total Uncertainty $\left(\Delta \rho^{*} \mathbf{H}_{\mathrm{m}}\right)=$ & \pm 121.44 \\
\hline
\end{tabular}




\subsubsection{Uncertainty in Energy Flow Rate}

Several flow measurement technologies may be used with the retrofit module, including orifice meters, turbine meters, and ultrasonic meters. Flow rate uncertainties for these meters have been reviewed to determine their effect on the accuracy of the energy meter module. The energy meter final output, i.e., the energy flow rate, is the product of the flow meter measurement of actual volumetric flow rate in combination with the actual volumetric heating value computed from the flowing density and mass-based heating value

$$
Q_{\text {energy }}\left(\frac{B t u}{d a y}\right)=Q_{v}\left(\frac{a c f}{d a y}\right) * \rho H_{m}\left(\frac{B t u}{a c f}\right)
$$

The sensitivity analysis discussed in the previous section yielded uncertainty predictions for the actual volumetric heating value $\left(\Delta \rho^{*} \mathrm{H}_{\mathrm{m}}=121.44 \mathrm{Btu} / \mathrm{acf}\right)$, based on input parameter uncertainties. These predictions can be used in combination with allowable uncertainty limits on the volumetric flow rate. The chosen volumetric flow rate uncertainty limits shown in Table 2.11 are based on AGA accuracy requirements for volumetric flow rate for each measurement technology.

Table 2.11. Accuracy limits on actual volumetric flow rate imposed by AGA standards.

\begin{tabular}{|c|c|c|}
\hline Metering Technology & $\begin{array}{c}\text { Standard establishing accuracy } \\
\text { limits on } Q_{v} \text { (actual) }\end{array}$ & $\begin{array}{l}\text { Accuracy limits at flowing } \\
\text { conditions }\end{array}$ \\
\hline Orifice flow meter & AGA-3 & $\begin{array}{l}\Delta Q_{v} / Q_{v}=0.0206 \text { (based on } \\
\text { error propagation of allowable } \\
\text { uncertainties in worst case) }\end{array}$ \\
\hline Turbine flow meter & AGA-7 & $\Delta Q_{v} / Q_{v}=0.01$ \\
\hline Ultrasonic flow meter & AGA-9 & $\begin{array}{l}\Delta Q_{v} / Q_{v}=0.007 \text { (large meter) } \\
\Delta Q_{v} / Q_{v}=0.014 \text { (small meter) }\end{array}$ \\
\hline
\end{tabular}

Since the volumetric flow rate measurement (determined by the specific meter technology) and the heat content (determined by the energy meter module) are independent of each other, the uncertainties may be estimated separately as

$$
\Delta Q_{\text {energy }}=Q_{v} * \Delta\left(\rho * H_{m}\right)+\rho * H_{m} * \Delta Q_{v}
$$

After dividing both sides by Qenergy, $_{\text {, the uncertainty may be expressed as }}$

$$
\frac{\Delta Q_{\text {energy }}}{Q_{\text {energy }}}=\frac{\Delta\left(\rho * H_{m}\right)}{\rho * H_{m}}+\frac{\Delta Q_{v}}{Q_{v}}
$$

To extend this uncertainty to indicate the total uncertainty in energy rate, a root-mean-square analysis may be used 


$$
\frac{\Delta Q_{\text {energy }}}{Q_{\text {energy }}}=\sqrt{\left(\frac{\Delta\left(\rho^{*} H_{m}\right)}{\rho^{*} H_{m}}\right)^{2}+\left(\frac{\Delta Q_{v}}{Q_{v}}\right)^{2}}
$$

Using a typical actual volumetric heating value of 45,000 Btu/acf, the energy content uncertainty for the worst-case condition is $\left(\Delta \rho * \mathrm{H}_{\mathrm{m}}\right) /\left(\rho^{*} \mathrm{H}_{\mathrm{m}}\right)=0.27 \%$. Energy content uncertainty and the highest allowable uncertainty in volumetric flow rate permitted by AGA (stated above) will yield the following errors for the energy meter module:
Orifice flow meter
$\Delta Q_{\text {energy }} / Q_{\text {energy }}=2.08 \%$
Turbine flow meter
$\Delta Q_{\text {energy }} / Q_{\text {energy }}=1.04 \%$
Ultrasonic flow meter (large)
$\Delta Q_{\text {energy }} / Q_{\text {energy }}=0.75 \%$
Ultrasonic flow meter (small)
$\Delta Q_{\text {energy }} / Q_{\text {energy }}=1.43 \%$

\subsection{Biases Due to Unmeasured Diluents}

The existing 6 percent algorithm was tested to estimate the errors due to common diluents besides nitrogen and carbon dioxide that are not measured or input to the algorithm. Finding the effects of these diluents on the algorithm accuracy is necessary because some of these diluents can appear in transmission-quality natural gas, or in other gases for which the energy meter may be used. For instance, the AGA-8 normal range of gases, described in Table 2.1, allows small amounts of helium, water, and hydrogen sulfide to be present. Typical diluent levels in transmission gases were also determined from discussions with several transmission companies and a recent report on gas quality [Interstate Natural Gas Association of America (1995)]. Typical industry tariff limits also allow significant amounts of oxygen in transmission gases. None of these diluents are explicitly measured by the energy meter, but they potentially can affect the speed-of-sound measurement and bias the heating value calculations.

To evaluate the effects of these additional diluents on algorithm accuracy, the algorithm was used to calculate standard volumetric heating values of gases with small amounts of the diluents present. In one set of calculations, various amounts of each diluent were added to Gas Composition B, described in the previous section, and reference properties of the diluted gases (including speed of sound) were computed using SonicWare ${ }^{\circledR}$ and GPA-2145. Standard volumetric heating values for the diluted gases were computed with the algorithm, using the SonicWare ${ }^{\circledR}$ speed-of-sound value and the diluted $\mathrm{CO}_{2}$ and $\mathrm{N}_{2}$ concentrations as input, but not otherwise accounting for the presence of the other diluent. The algorithm results were compared to the reference values to determine how the algorithm errors in $\mathrm{H}_{\mathrm{v}}$ depend on the mol\% of each diluent. Another set of calculations determined the error trends in $\mathrm{H}_{\mathrm{v}}$ when each diluent was added to pure methane. The errors were more severe for the tests with pure methane as the base gas, so diluent limits were chosen using the methane results. Table 2.12 lists the maximum amount of each diluent that limits the error in $\mathrm{H}_{\mathrm{v}}$ to within $\pm 0.25 \%$, and compares these limits to the AGA-8 normal range limits and typical tariff limits in the gas transmission industry. 
Table 2.12. Comparison of algorithm limits on unmeasured diluents with limits of AGA-8 normal range and typical tariff limits.

\begin{tabular}{|c|c|c|c|c|c|}
\hline Diluent & $\left.\begin{array}{c}\text { Error in } \mathbf{H}_{\mathbf{v}} \\
\text { \% error }\end{array}\right)$ & $\begin{array}{c}\text { AGA-8 normal } \\
\text { range limit } \\
\text { mol\% diluent }\end{array}$ & $\begin{array}{c}\mathbf{H}_{\mathbf{v}} \% \text { error } \\
\text { in AGA-8 } \\
\text { normal range }\end{array}$ & $\begin{array}{c}\text { High tariff } \\
\text { limit (mol\%) }\end{array}$ & $\begin{array}{c}\mathbf{H}_{\mathbf{v}} \text { \% error } \\
\text { in high tariff } \\
\text { limit }\end{array}$ \\
\hline $\mathrm{H}_{2} \mathrm{~S}$ & 1.0 & 0.02 & 0.02 & 0.0016 & 0.0016 \\
\hline $\mathrm{H}_{2} \mathrm{O}$ & 1.1 & 0.05 & 0.06 & 0.015 & 0.016 \\
\hline $\mathrm{O}_{2}$ & 1.67 & 0 & 0 & 1.0 & 1.67 \\
\hline $\mathrm{CO}$ & -0.23 & 0 & 0 & - & - \\
\hline $\mathrm{H}_{2}$ & 0.15 & 0 & 0 & - & - \\
\hline $\mathrm{He}$ & 0.25 & 0.2 & 0.05 & - & - \\
\hline
\end{tabular}

The analysis of the algorithm revealed that diluents within the normal range of AGA-8 would cause acceptable errors $\left(<0.06 \%\right.$ in $\left.\mathrm{H}_{\mathrm{v}}\right)$. Hydrogen sulfide and water vapor content at or below typical tariff limits will not cause significant errors. This is an advantage, since tariff limits, not the AGA-8 standard, are often the principal factor used by transmission companies for accepting natural gas from producers. Tariff limits on oxygen, however, are higher than the normal range and could allow enough oxygen in the stream to cause unacceptable errors in the calculated heating value of more than $1.5 \%$. Based on this diluent analysis of the algorithm, the existing 6 percent algorithm must be limited to transmission gases that fall within the AGA-8 normal range, and no changes should be made to include other diluents at this time.

Based on these results, application testing of the energy meter module, in combination with the 6 percent algorithm, should be limited to transmission-quality gases. Future work should endeavor to tailor the 20 percent algorithm (discussed in the next section) to production gases and possible diluent concentrations exceeding those in the AGA-8 normal range.

\subsection{Extension of Algorithm to Higher Diluent Contents}

In its present form, the 6 percent algorithm predicts the energy content and density of natural gas mixtures that fall within pipeline transportation tariffs for gas quality. The limit of allowable diluent concentrations may potentially be increased from 6 percent to 20 percent by modifying the correlations for $\mathrm{N}_{2}$ and $\mathrm{CO}_{2}$ concentrations. This would lower the allowable range of standard heating values below $1000 \mathrm{Btu} / \mathrm{scf}$.

To test the feasibility of a modified correlation in diluents, data were collected on gases with diluent concentrations greater than 6 percent. The original GERG database that provided data for the "6 percent algorithm" [Starling et al. (1991)] was used to obtain gas compositions with nitrogen or carbon dioxide concentrations up to $20 \%$ for use in a new correlation database. Data were also obtained on discovered and undiscovered U.S. natural gas resources, including sour gases and unprocessed production gases [Gas Research Institute (1998)]. Gases from this reference that met the criteria of up to $20 \% \mathrm{~N}_{2}$ and/or $20 \% \mathrm{CO}_{2}$ were also added to the "20 percent database." Most of these gases had higher nitrogen content than carbon dioxide content, so to avoid bias toward nitrogen in the correlations, a second version of each gas was created by interchanging the original $\mathrm{N}_{2}$ and $\mathrm{CO}_{2}$ concentrations. The final $20 \%$ database contained 834 
gases composed of hydrocarbons (methane through octane), up to 20 percent nitrogen, up to 20 percent carbon dioxide, and lesser amounts of hydrogen sulfide, helium, hydrogen, carbon monoxide, and oxygen. Table 2.13 lists the ranges of mol\% of each gas in the database. To ensure that subsequent algorithm enhancements would be valid up through $20 \%$ nitrogen and carbon dioxide and would not require extrapolation to these levels, two gases were included in the database containing $23.7 \% \mathrm{~N}_{2}$ and $23.7 \% \mathrm{CO}_{2}$, respectively.

Table 2.13. Compositions (in mol\%) of gases in 20 percent algorithm database.

\begin{tabular}{|l|c|c|}
\hline Component & Minimum mol\% & Maximum mol\% \\
\hline Methane & 44.8178 & 99.3497 \\
\hline Ethane & 0 & 42.3 \\
\hline Propane & 0 & 10.0939 \\
\hline Isobutane & 0 & 2.7084 \\
\hline n-Butane & 0 & 4.3009 \\
\hline Isopentane & 0 & 1.7982 \\
\hline n-Pentane & 0 & 2.1726 \\
\hline n-Hexane & 0 & 9.2165 \\
\hline n-Heptane & 0 & 4.6083 \\
\hline n-Octane & 0 & 1.5361 \\
\hline Nitrogen & 0 & 23.732 \\
\hline Carbon Dioxide & 0 & 23.732 \\
\hline Hydrogen Sulfide & 0 & 30.3416 \\
\hline Helium & 0 & 1.1002 \\
\hline Oxygen & 0 & 1.2601 \\
\hline Carbon Monoxide & 0 & 0.9142 \\
\hline Hydrogen & 0 & 35.6310 \\
\hline
\end{tabular}

Molecular weights and mass-based heating values of the 20 percent database gases were computed using the latest revision of GPA Standard 2145 [Gas Processors Association (2000)]. SonicWare ${ }^{\circledR}$ and the FORTRAN code SUPERZ [Savidge (1989)] were used to compute density and sound speed of the gases. SUPERZ is an early, modified version of the AGA8PROG code [American Gas Association (1994)] that computes density, speed of sound, and other useful properties from the AGA-8 equations of state. SUPERZ was chosen over SonicWare ${ }^{\circledR}$ to compute properties of most of the database gases because the program could easily be adapted to batch-mode calculations on hundreds of gas compositions. To ensure consistency, SUPERZ was validated against SonicWare ${ }^{\circledR}$ by comparing density and speed-of-sound values at STP from the two programs.

Properties were computed, using both codes, for those gases in the database having the maximum content of each component as shown in Table 2.13. Comparison of the results revealed those components in the database for which properties and interaction parameters in SUPERZ did not agree with SonicWare ${ }^{\circledR}$. Next, calculations were performed on binary mixtures of methane and each component that caused disagreement. These calculations were used to find 
the highest concentration of each component for which SUPERZ and SonicWare ${ }^{\circledR}$ results agreed to within $0.005 \%$. These concentration limits, shown in Table 2.14, were chosen as breakpoints for property calculations. For those few gases in the 20 percent database where gas components were above the breakpoints, SonicWare ${ }^{\circledR}$ values were used, while SUPERZ values were used for the remaining majority of the database. This approach allowed densities and sound speeds of the database gases to be computed quickly and consistently. New correlations were performed on the property data for the 20 percent database gases.

Table 2.14. Component limits, in binary mixtures with methane, above which observed disagreement between SUPERZ and SonicWare ${ }^{\circledR}$ exceeds $\mathbf{0 . 0 0 5 \%}$.

\begin{tabular}{|c|c|c|}
\hline Component & $\begin{array}{l}\text { Upper limit } \\
\text { (mol\%) }\end{array}$ & $\begin{array}{l}\text { Property in which } \\
\text { disagreement occurs }\end{array}$ \\
\hline $\mathrm{n}$-Hexane $\left(\mathrm{C}_{6}\right)^{*}$ & 1 & density \\
\hline $\mathrm{n}$-Heptane $\left(\mathrm{C}_{7}\right)^{*}$ & 1 & density \\
\hline $\mathrm{n}$-Octane $\left(\mathrm{C}_{8}\right)^{*}$ & 1 & density \\
\hline $60 / 30 / 10 \mathrm{mix}$ of $\mathrm{C}_{6} / \mathrm{C}_{7} / \mathrm{C}_{8}{ }^{*}$ & 1.5 & density \\
\hline Hydrogen Sulfide & 5 & density \\
\hline Carbon Monoxide & $>0$ & speed of sound \\
\hline Hydrogen & 12 & density \\
\hline \multicolumn{3}{|c|}{$\begin{array}{l}\text { *Trends in density disagreements for hexane alone are in the opposite } \\
\text { direction of trends for heptane and octane alone. Properties computed witl } \\
\text { SUPERZ for gases with hexanes-plus mixtures agree with SonicWare }{ }^{\circledR} \text { to } \\
\text { higher limits. }\end{array}$} \\
\hline
\end{tabular}

The algorithm gas parameters were computed by SonicWare ${ }^{\circledR}$ and SUPERZ for each gas composition, including $\mathrm{CO}_{2}, \mathrm{~N}_{2}$, and the other specified diluents to derive proper heating value correlations. These supplemental diluents are not independent variables in the correlations, however, because they are not currently measured by the energy meter module and cannot be input to the energy meter algorithm. This can lead to inaccuracies in the 20 percent algorithm.

Initially, because the 20 percent algorithm was intended for distribution-quality gases, only those gases in the database that meet the common tariff limits of Table 2.12 were used in the curve fits. Of the 834 gases in the database, 550 met these criteria and were fit to the new correlations. However, checks of the heating value correlation revealed disagreements between the data and the correlation approaching 1\% (Figure 2.8). Further analysis revealed that the residuals were strongly correlated to the presence of oxygen (Figure 2.9). Oxygen is allowed by tariffs in quantities up to $1 \%$, but is not measured by the energy meter or explicitly entered into the regressions. To better evaluate the effect of oxygen, another correlation was performed to account for oxygen concentration, although the energy meter does not currently measure it. As Figure 2.10 shows, this three-diluent correlation computes heating values of most database gases to within $\pm 0.05 \%$, although some residuals approach $+0.35 \%$. These results strongly suggest that oxygen must be measured and included in the correlation of heating value for the energy meter to be accurate in gas environments containing oxygen.

The high residuals for some gases in Figure 2.10 suggest that other effects on heating value are still not adequately accounted for, possibly related to diluents that are not included in 
the algorithm. To reduce the contributions of other unaccounted-for diluents, another subset of the 20 percent database was created, containing only 146 gases that fall within the AGA-8 normal range (Table 2.1). Hydrogen, carbon monoxide, and oxygen were eliminated from this subset, and the maximum mol\% of other diluents (except for $\mathrm{N}_{2}$ and $\mathrm{CO}_{2}$ ) were significantly reduced from the first subset based upon tariff limits. Data from this AGA-8 normal range subset were correlated without including oxygen as an independent variable. The new correlations reproduced gas properties more accurately than the earlier correlations for data having higher unaccounted-for diluent content. As shown in Figures 2.11 and 2.12, densities at STP and massbased heating values were reproduced to within $\pm 0.05 \%$ by these correlations.

In summary, to accurately measure heating values of production gases, the energy meter must detect other diluents besides nitrogen and carbon dioxide. These could be detected by direct measurement or inferred through the measurement of other mixture properties. The "20 percent algorithm" should be modified to include the effects of these additional diluents.

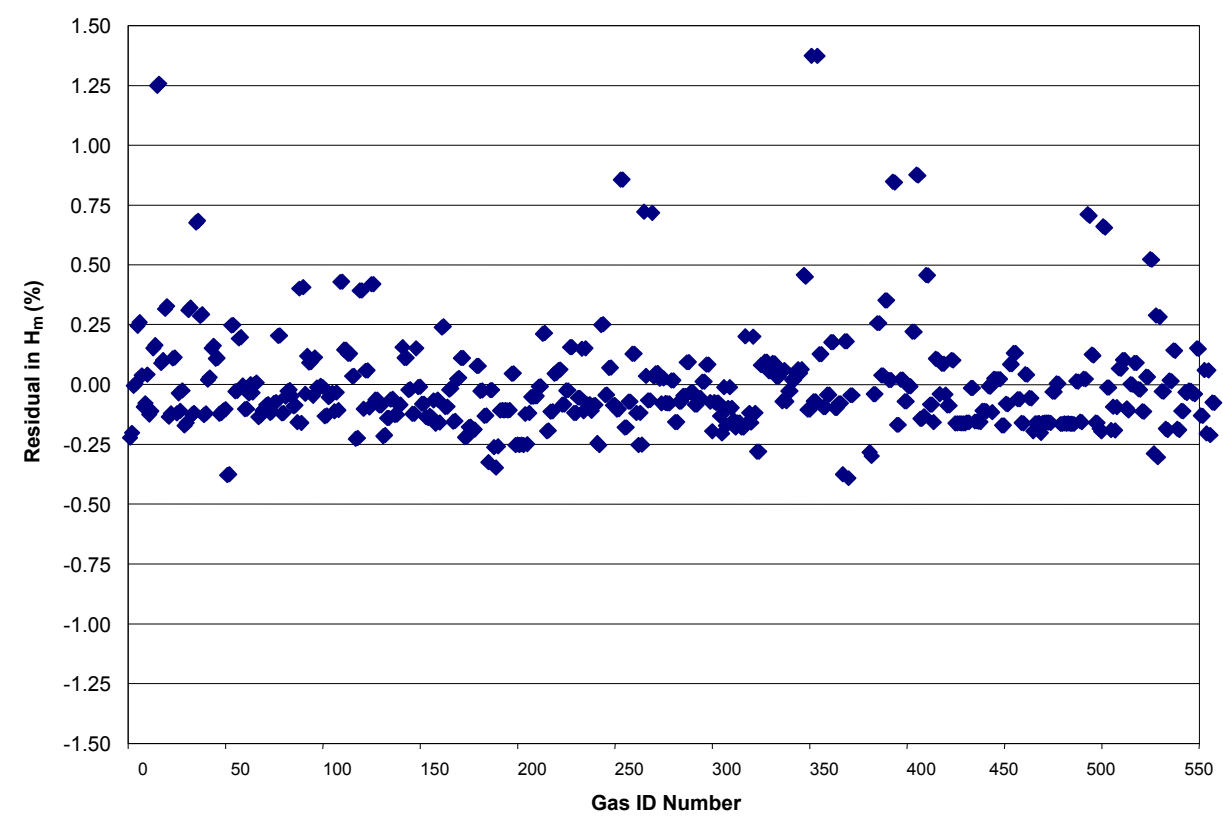

Figure 2.8. Residuals in improved correlation to mass-based heating values of 20 percent database gases within tariff limits. 


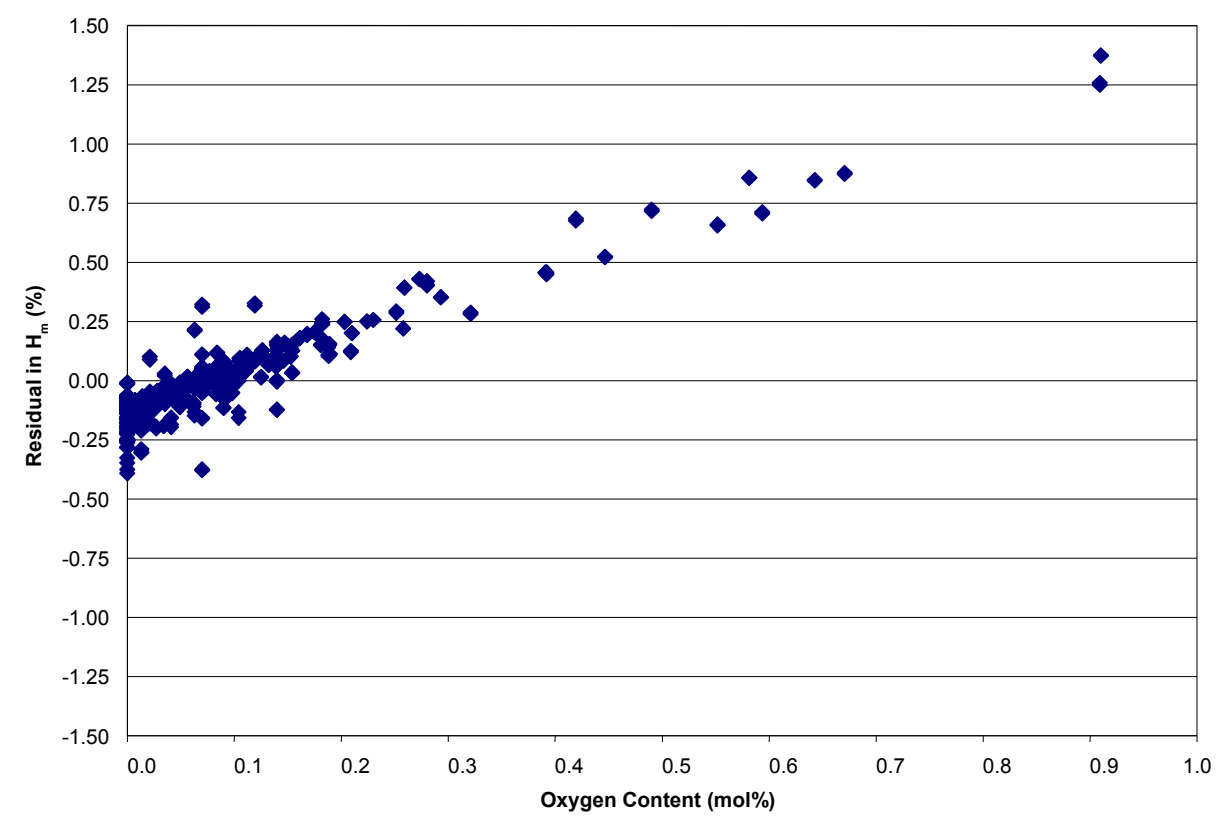

Figure 2.9. Dependence of improved correlation residuals on oxygen content of correlated gases.

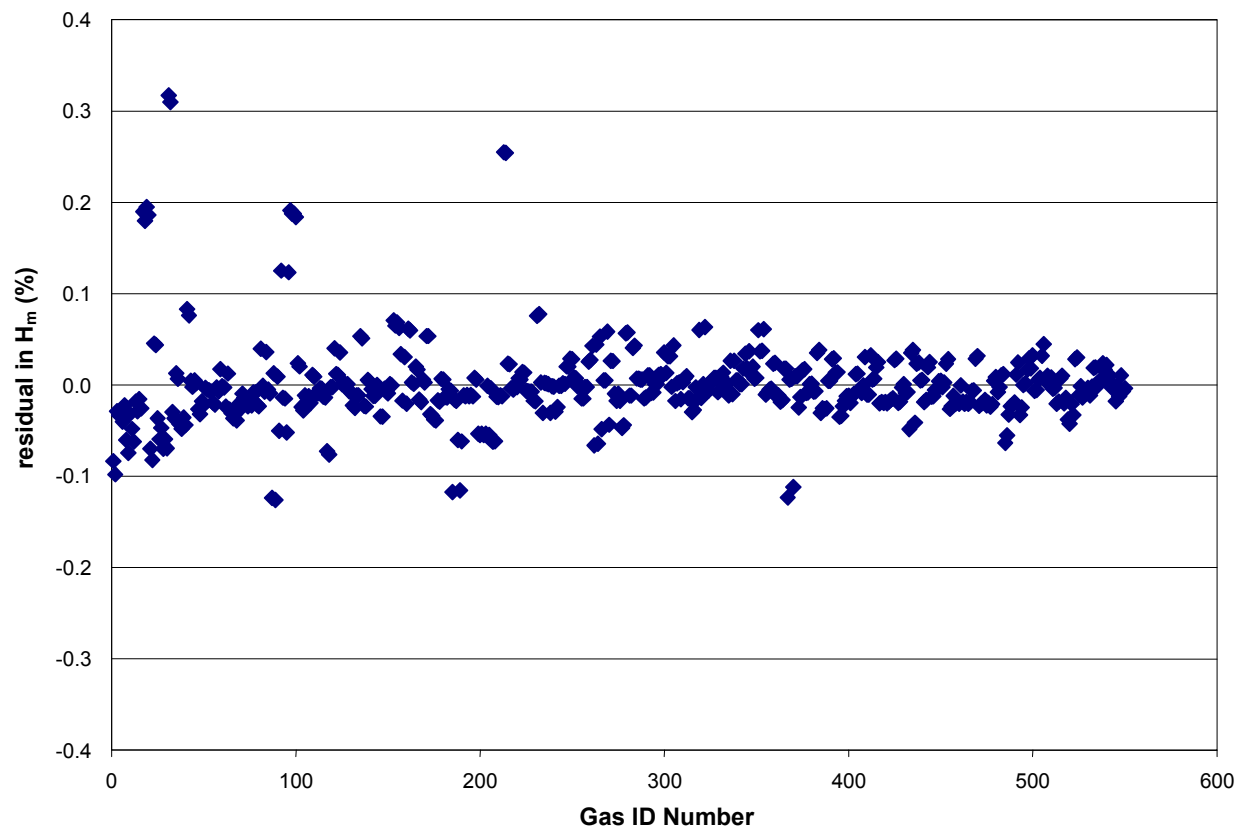

Figure 2.10. Residuals in improved correlation to mass-based heating values when oxygen is included. 


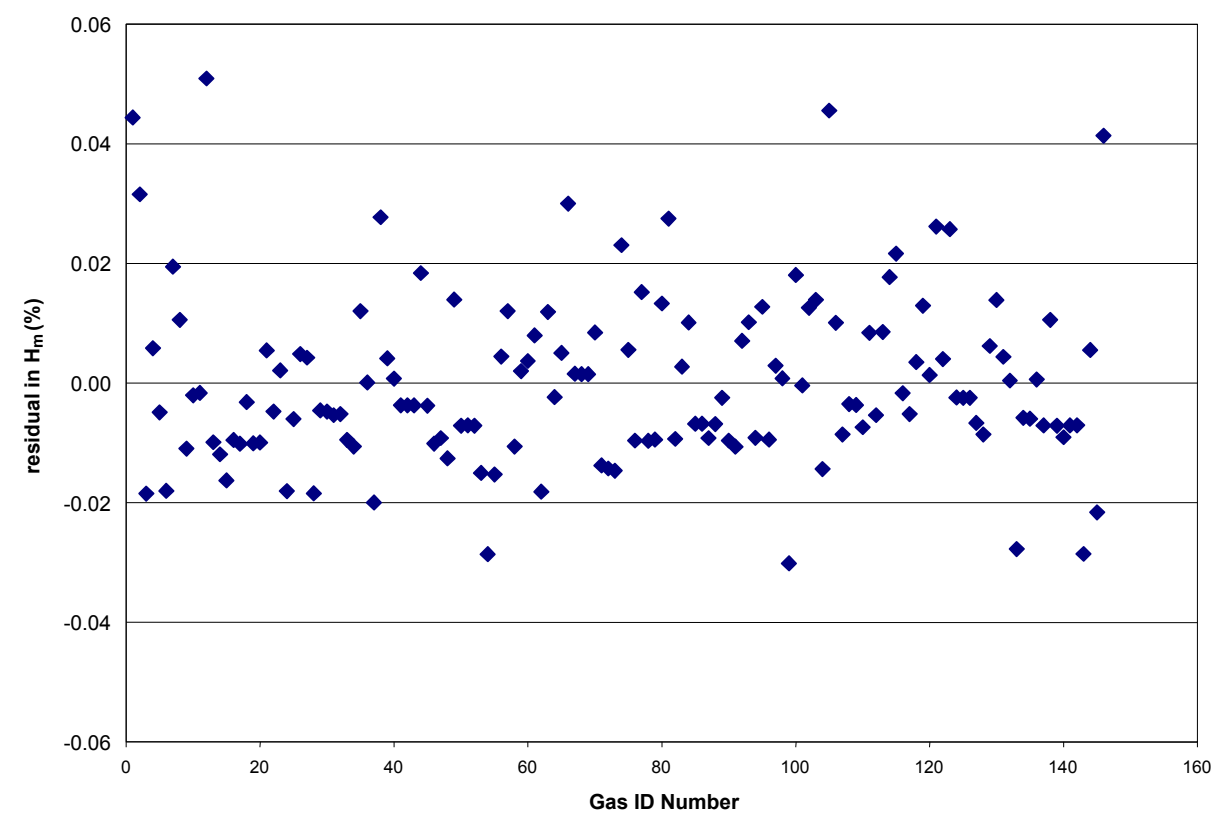

Figure 2.11. Residuals in improved correlation to mass-based heating values of 20 percent database gases within AGA-8 normal range limits.

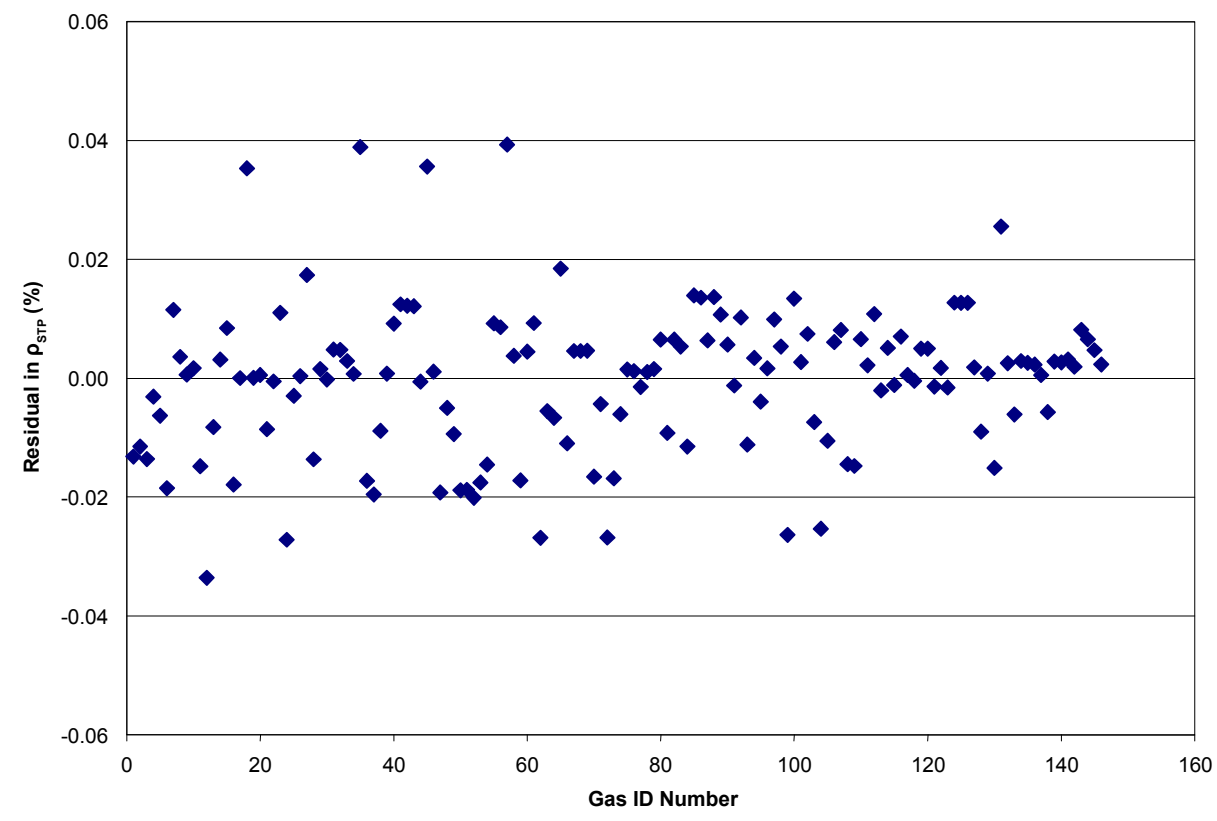

Figure 2.12. Residuals in improved correlation to densities at STP of 20 percent database gases within AGA-8 normal range limits. 


\subsection{Module Improvements}

\subsection{Overview}

Improvements to the Energy Meter Module during 2000-2001 include:

Speed-of-sound sensor: Analysis of ultrasonic pulse time-of-flight measurement sensitivity to near-field phase error, pressure and temperature measurement accuracies, thermal expansion effects in the two-reflector target, and the approximate effects of ultrasonic attenuation and dispersion in the gas. This work provides technical insight into the operation of the ultrasonic measurement method and the magnitude of possible associated errors. A principal result is the definition of the optimum ultrasonic frequency range and bandwidth of operation and the digital waveform sampling and signal processing accuracy requirements.

Gas temperature measurement and control: Experimental tests of the 1999-2000 speedof-sound chamber to determine the cause of discrepancies in accurately determining the gas temperature and to identify test chamber design changes required to improve the gas temperature measurement and control. Results of this effort led to an accurate method of gas thermal equilibration to match the ambient temperature of the test chamber.

Bench tests of ultrasonic transducers: Experimental tests of five commercially available ultrasonic transducers having operating frequencies of $200 \mathrm{kHz}, 220 \mathrm{kHz}, 500 \mathrm{kHz}, 750 \mathrm{kHz}$, and $279 \mathrm{kHz}$ in several natural gas mixtures under controlled static conditions. These tests demonstrate the importance of transducer operating frequency and bandwidth as well as the pressure-dependent effects of acoustic coupling of sound energy into the gas and the frequency effects of sound attenuation by molecular relaxation, viscosity, and heat conduction in the gas. A custom-designed ultrasonic transducer operating at a frequency of $266 \mathrm{kHz}$ and having a bandwidth of $128 \mathrm{kHz}$ gave the best performance. (Refer to the appendix of this report for details of the bench test results.)

New speed-of-sound test chamber: Design and fabrication of a new speed-of-sound test chamber by which the body of the test chamber serves as a heat sink to bring the gas to the same temperature as the chamber prior to entering the chamber for measurement and using a low thermal expansion material as the two-reflector target.

New carbon dioxide test chamber: Design and fabrication of a carbon dioxide sensor chamber to accommodate the use of a commercial nondispersive infrared $\mathrm{CO}_{2}$ sensor incorporating the same gas thermal equilibration technique as that used in the speed-of-sound chamber.

Laboratory tests of new sensor module: Experimental tests of improved speed-of-sound and $\mathrm{CO}_{2}$ sensors under MRF flow loop conditions. These tests revealed the sensors to have speed-of-sound and $\mathrm{CO}_{2}$ measurement accuracy and robust performance sufficient to meet the gas energy measurement target accuracy under outdoor summer environmental conditions when operating in a range of natural gas mixtures.

Nitrogen sensing techniques: Evaluation of four conceptual inferential methods for sensing diluent nitrogen in natural gas. Although some of the methods reviewed are technically sound, insufficient development work is available on those methods to indicate their feasibility for determining nitrogen content to within the accuracy required by the gas heat energy algorithm. 
Technical efforts and detailed results on all of these study tasks are presented below.

\subsection{Speed-of-Sound Sensor}

\subsubsection{Basic Concept and Approach}

Practical sensor techniques for measuring the speed of sound in natural gas in industrial field settings typically utilize piezoelectric transducers to generate and detect sound waves in the frequency range of about $100 \mathrm{kHz}$ to $1 \mathrm{MHz}$. The lower frequency limit is governed by the accuracy and resolution that may be attained in measuring the transit times of the ultrasonic signals and the upper frequency limit is restricted by viscous absorption of the ultrasonic waves propagating in the gas. For example, commercial ultrasonic gas flow meters operate in the lower part of this frequency range using separate source and detector transducers to detect the transit times of sound pulses traveling along several spatial paths directed upstream and downstream through the flowing gas. This measuring technique yields both the volumetric flow velocity of the transported gas and the speed of sound in the flowing gas. Pulsed ultrasonic signals are used as a convenient method for directly measuring the travel times of the sound waves along known paths a few feet in length oriented obliquely across the diameter of the pipe.

Smaller scale devices dedicated exclusively to the precision measurement of speed of sound in natural gas are also practical using ultrasound transducers operating at higher frequencies, typically in the range of $500 \mathrm{kHz}$, and employing short sound propagation paths only a few inches in length. Sensor arrangements using this approach may determine the speed of sound from tests on a sample of the gas in question performed at a pressure and temperature different from the flowing conditions in the source pipeline. However, in order to achieve precision measurement results on such a small scale, added attention must be given to the accuracy by which the ultrasonic pulse transit time is measured, the accuracy and stability of the propagation path, and the homogeneity of the gas sample volume during the transit time measurements, among other possible sources of error. First, since the transit time measurement is a primary source of error, the ultrasonic pulse waveform must be well defined in time by having a fast rise time and a stable wave shape to allow accurate and consistent measurements relative to the pulse initiation time at the source. Second, the sound propagation path length must either be essentially independent of thermal expansion effects or otherwise compensated for such effects by means of appropriate temperature measurements of the gas sample test chamber structure. Additionally, the gas flow through the test chamber should be designed to prevent any stagnation of the gas in order to ensure that the speed-of-sound measurement is representative of the current gas sample under test.

The speed-of-sound sensor designed for the 1999-2000 tests utilized a single transmitreceive ultrasonic transducer operating at a frequency of $200 \mathrm{kHz}$ and a dual reflector having a 1-in. spacing between the reflecting faces. The transducer and target were contained within a small-scale test chamber. This arrangement has several advantages over conventional twotransducer methods, particularly with regard to accurate transit time measurements and minimal thermal effects on the sound propagation path length over which the transit time is measured. The primary advantage is that the two received reflected-pulse waveforms may be compared to derive the sound wave transit time instead of comparing the time of arrival of one received pulse waveform with the transmit trigger pulse that initiated the sound pulse. The second advantage is that the dual reflector target may be made from a single piece of material to minimize mounting 
uncertainties and temperature-dependent dimensional changes in the distance between the two reflectors. The test chamber was designed to be well ventilated by the in-flowing gas via several gas injection inlets located at different positions and angles on the chamber body. An RTD probe sensor extending into the central volume of the chamber measured gas temperature. A crosscorrelation method was used to accurately compare the two reflected signals to yield their time delay in a manner independent of the reflector target distance from the transducer and also independent of the transmitted waveform trigger pulse. Bench tests performed on this sensor design using nitrogen gas at room temperature and pressures up to 45 psia showed that speed-ofsound measurements could be obtained to within less than $\pm 1 \mathrm{ft} / \mathrm{sec}$ of the theoretical values. The ultrasonic transducer was operated at its resonance frequency of $200 \mathrm{kHz}$ and it had a $-6 \mathrm{~dB}$ bandwidth of approximately $20 \mathrm{kHz}(10 \%)$. The dual-reflector target had planar reflecting faces located at 2 in. and 3 in., respectively, away from the face of the ultrasonic transducer.

\subsubsection{Speed-of-Sound Test Results, 1999-2000}

The speed-of-sound sensor was tested in the MRF Low Pressure Loop (LPL) to evaluate its performance in flowing natural gas. Data recorded for this purpose consisted of pressure and temperature in the gas sample test chamber and the two reflected ultrasonic waveforms digitally sampled and recorded as time series signals. Gas that flowed through the speed-of-sound sensor was maintained at a regulated pressure of 30 psia and had a temperature in the range of $70-90^{\circ} \mathrm{F}$. Speed-of-sound results were derived from these test data (two-way travel distance between the reflectors divided by the measured transit time) and compared with known reference values of speed of sound calculated via the AGA-8 equation of state using SonicWare ${ }^{\circledR}$. Data for these calculations included the measured test chamber pressure and temperature in combination with the LPL gas composition data obtained using a Daniel gas chromatograph (GC).

For typical speed-of-sound values in the range of 1400-1450 ft/sec at the gas temperatures and pressures tested, the target measurement accuracy of $\pm 1.5 \mathrm{ft} / \mathrm{sec}$ calls for a sensor accuracy of $\pm 0.11 \%$. The LPL test results showed that the overall error in measured speed of sound was consistently greater than this required error limit and ranged as high as $4 \%$ for many of the measurements.

The individual measurements contributing to the experimental speed-of-sound values were evaluated in order to ascertain the sources of error. For the computed speed of sound, the SonicWare $^{\circledR}$ calculations used the measured chamber pressure and temperature, and the gas composition from the Daniel GC. Gas composition from the GC is generally accurate and its negligible errors were disregarded as a potential source of error in the computed speed of sound. The pressure measurement in the test chamber proved to be highly reliable, and errors in pressure were considered insignificant. However, the gas temperature sensor appeared to measure the temperature of the chamber housing rather than the temperature of the gas in the chamber. Furthermore, the throughput flow and mixing of the gas in the chamber appeared to be assisting in the heat transfer between the test chamber housing and the test gas sample resulting in thermal gradients within the chamber. Therefore, establishing uniform gas temperature in the chamber and obtaining an accurate measurement of the true gas temperature associated with the speed-ofsound measurements was identified as a critical design factor to be corrected. 
The measurement of speed of sound of the gas involved the distance between the two reflecting targets and the time difference between the two received reflection signals. The distance between the two reflectors, made from a single piece of metallic material, was carefully measured and was not considered to be a major source of error.

The measured transit time between the two reflected signals $(\Delta t)$ was a second source of error in the speed of sound. An examination of the recorded ultrasonic waveforms revealed that the cross-correlation function contained many oscillatory cycles and peaks of nearly equal amplitude. The time delay between the two pulse signals, indicated by the cross-correlation time lag at the central positive lobe having the maximum amplitude, was often difficult to distinguish from the adjacent lobes. A small amount of ultrasonic or electronic noise in the recorded waveforms could easily cause the signal processing calculations to select the wrong correlation lobe causing a potential transit time error equal to one period of the ultrasonic wave. This cycle shift error is primarily caused by the narrow bandwidth of the ultrasonic transducer used in the measurements together with weak received ultrasonic pulse waveforms caused by attenuation of both reflections along the 4- and 6-in. two-way reflection paths. Therefore, the signal processing procedure could miss the maximum correlation lobe by one complete cycle when performing the lag time selection process. The measurement errors in speed of sound were significantly reduced by adjusting the experimental two-reflector transit time measurement by one cycle (a time delay of $5 \mu \mathrm{s}$ ) in the calculated cross-correlation functions.

The digital sampling rate used in recording the reflected ultrasonic pulses can produce errors in the time resolution of the correlation function peak value. If the indicated peak value of the cross-correlation peak is in error by one sample time interval, a corresponding shift will appear in the time delay measurement. Therefore, the digitization rate must over-resolve the ultrasonic pulse waveforms in order to achieve the timing accuracy needed for the precision speed-of-sound measurements.

The 2000-2001 task efforts to improve the speed-of-sound measurement focused on reducing the most prominent sources of error: (1) gas temperature control and measurement; and (2) accurate and reliable time measurement between the two reflected signals.

\subsubsection{Speed-of-Sound Measurement Methods}

Two common techniques used to measure speed of sound are the pitch-catch and pulseecho methods. In the pitch-catch method, two ultrasonic transducers are oriented face to face at a known separation distance. One transducer is used to generate an ultrasonic pulse and the other transducer is used to receive the pulse. By knowing the distance between the two transducers and measuring the elapsed time between initiating the pulse and detecting the pulse, the speed of sound can be determined in the medium between the two transducers. Figure 3.1 shows a simplified schematic drawing of this method. As the figure shows, a known distance D separates the transducers. 


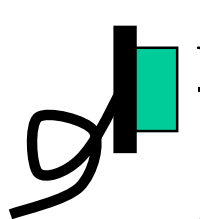

Transmitted Pulse

$$
\left(t=t_{0}\right)
$$

Gas

\section{$D$}

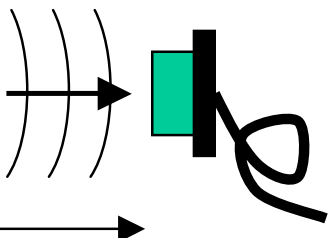

Received Pulse

$\left(t=t_{d}\right)$

Figure 3.1. Schematic drawing of pitch-catch method.

If $t_{d}-t_{0}$ is the time required for an ultrasonic pulse to travel from the transmit transducer to the receiving transducer, then the speed of sound is

$$
v_{g a s}=\frac{D}{t_{d}-t_{0}}
$$

where $v_{\text {gas }}$ is the speed of sound in the gas medium between the two transducers.

Although this method requires two transducers, it has the advantage of being very simple to set up. Furthermore, the pulse received generally has a good signal-to-noise ratio because the ultrasonic signal travels directly from the transmitting transducer to the receiving transducer without any loss associated with reflections.

In the pulse-echo method, a single transducer is used as the transmitter and the receiver. Figure 3.2 shows a simplified schematic drawing of the pulse-echo method. With this method, the generated wave is reflected from a planar target located at a known distance from the transducer and is received by the same transducer.

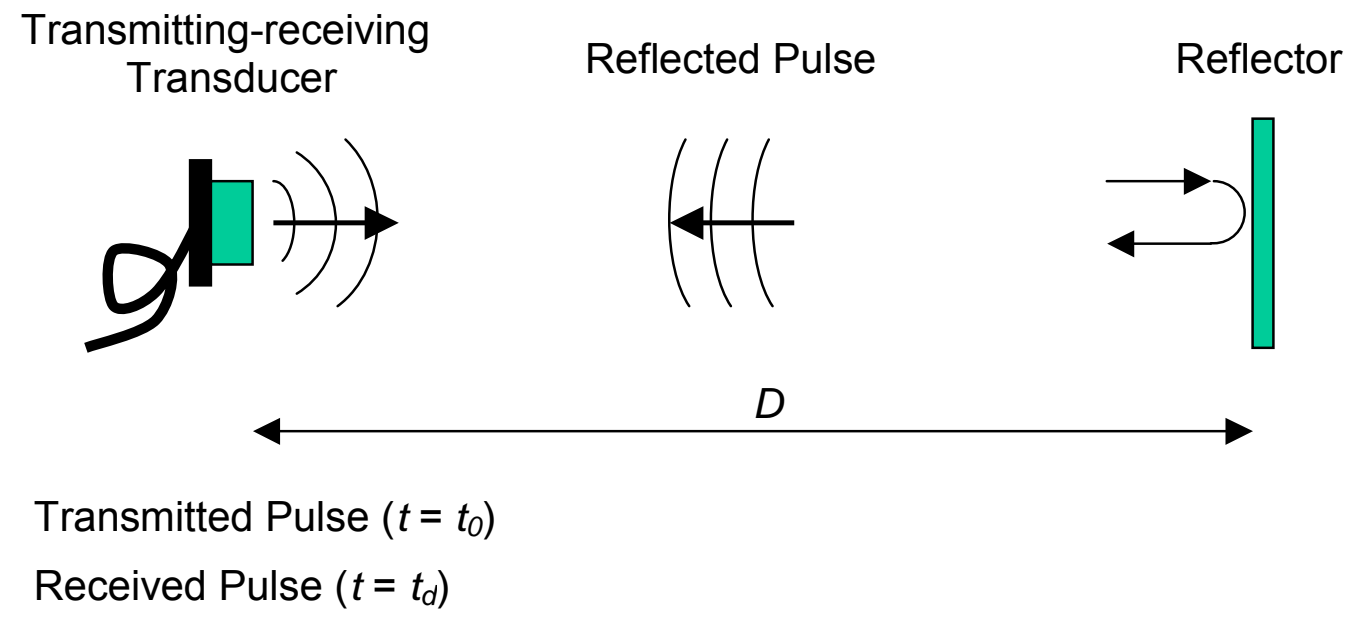

Figure 3.2. Schematic drawing of pulse-echo method. 
If the distance between the transducer and the reflector is $\mathrm{D}$ and the measured time delay between the transmit trigger pulse and the detected pulse is $t_{d}-t_{0}$, then the speed of sound is

$$
v_{\text {gas }}=\frac{2 D}{t_{d}-t_{0}}
$$

The pulse-echo method is also very simple to apply and has the advantage of requiring only one transducer. However, the reflected pulse is weaker than in the pitch-catch method because of the reflection loss at the reflector and the longer two-way distance of travel.

Both of these methods have the disadvantage of inherent errors caused by uncertainties in the separation distance, $\mathrm{D}$, and uncertainties in the pulse initiation time, $\mathrm{t}_{0}$, and pulse detection time, $t_{d}$. In a permanent setup, the distance, $D$, can be accurately measured and may also be compensated for systematic changes caused by thermal expansion. However, there is still an uncertainty in the exact position at the face of the transducer where signals change from mechanical vibrational energy in the transducer to acoustical energy in the gas. Nevertheless, this uncertainty can be removed by calibrating the effective propagation path length using a gas medium with an accurately known speed of sound.

The uncertainty in the elapsed pulse travel time, $t_{d}-t_{0}$, stems from the finite reaction time for the acoustic pulse to be initiated after applying the electrical excitation pulse at $t=t_{0}$ and the fact that the exact time instant at which the received pulse is detected is a relatively arbitrary choice. The combination of these two uncertainties in time is referred to as the "Time Delay Error" (TDE).

\subsubsection{Two-Reflector Measurement Method}

To eliminate or reduce the TDE in the speed-of-sound measurement, an alternative method was devised. The method is an adaptation of the pulse-echo method using pulse signals received from two reflectors. Figure 3.3 shows the essential features of the method.

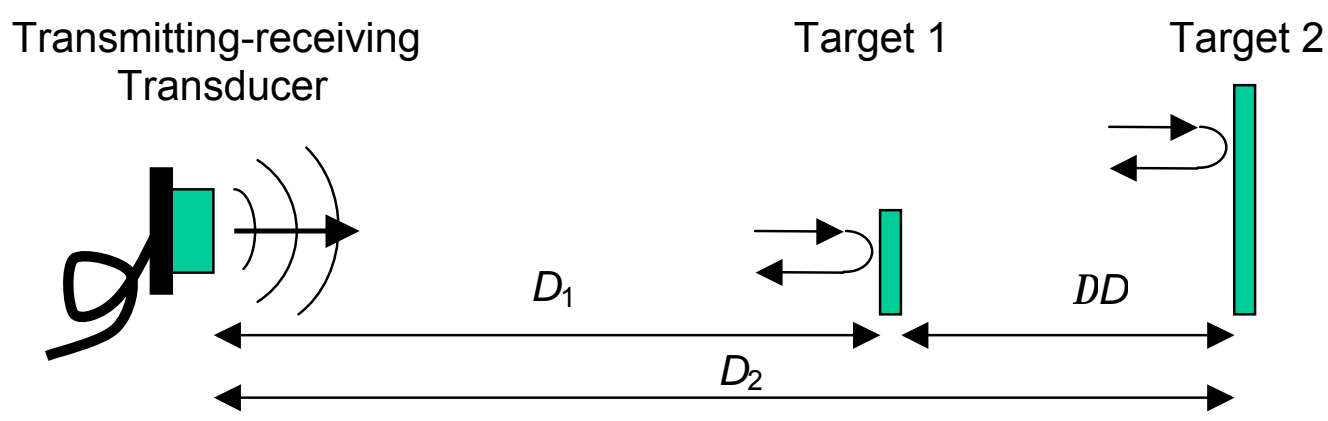

Figure 3.3. Schematic drawing of two-reflector pulse-echo method. 
The true time of arrival of the reflected pulse from Target $1, \mathrm{t}_{1}$, can be written as

$$
t_{1}=\frac{2 D_{1}}{v_{\text {gas }}}
$$

If the reflected pulse transit time were measured using the basic pulse-echo method, the apparent time of arrival from Target $1, \mathrm{t}_{1 \text { measured, }}$ would be

$$
t_{1 \text { measured }}=t_{1}+\mathrm{TDE}
$$

Similarly, for reflections from Target 2 , the true time of arrival is

$$
t_{2}=\frac{2 D_{2}}{v_{\text {gas }}}
$$

and the apparent measured travel time of the reflected signal from Target $2, \mathrm{t}_{2 \text { measured }}$, would be

$$
t_{2 \text { measured }}=t_{2}+\mathrm{TDE}
$$

As mentioned previously, the TDE (for both reflected signals) consists of two uncertainties. The uncertainty in the starting point of the initial pulse signal, $t_{0}$, will be the same for both reflection measurements. The second uncertainty in TDE is the point in time on the pulse waveform where detection is designated to occur. In general, the reflected waveforms from the two targets will be similar. If the time of arrival is measured at the same position on both waveforms, the second

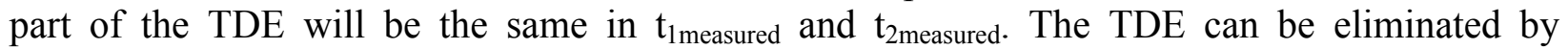
subtracting Equation (3.4) from Equation (3.6), thus canceling the TDE components

$$
\Delta t=t_{2 \text { measured }}-t_{1 \text { measured }}=t_{2}-t_{1}
$$

By combining Equations (3.3), (3.5), and (3.7), the time difference, $\Delta \mathrm{t}$, can be expressed in terms of distance and speed of sound

$$
\Delta t=t_{2}-t_{1}=\frac{2\left(D_{2}-D_{1}\right)}{v_{\text {gas }}}=\frac{2 \Delta D}{v_{\text {gas }}}
$$

and on rearranging, the speed of sound is

$$
v_{g a s}=\frac{2 \Delta D}{\Delta t}
$$


where $\Delta \mathrm{D}$ is the known distance between the two targets, and $\Delta \mathrm{t}$ is the time difference between the two received signals. This method has proven to be the most reliable way to measure the speed of sound accurately. In Equation (3.9), the TDE has been eliminated. Other sources of error, however, exist in the speed-of-sound measurement and are discussed in further detail below.

\subsubsection{Evaluation of Speed of Sound Measurement Characteristics}

\subsubsection{Ultrasonic Beam Diffraction}

In the speed-of-sound chamber, the ultrasonic waves interact with the surrounding walls as well as with the two targets, and multiple reflections may occur. Some of the waves reverberating from the chamber walls will be returned to the transducer, producing unwanted interference in the waveform. The near-field effects of the transducer can also influence the reflections of ultrasonic waves from the two targets.

To study the propagation of ultrasound from a transducer, a computer simulator using the Multi-Gaussian Beam Model was performed [Minachi et al. (1997)]. This simulator is a threedimensional beam model that can predict the near field and far field radiation patterns from an ultrasonic transducer. By simulating the radiation patterns of the various transducers available to the project, the chamber dimensions and reflector spacing were sized to achieve optimum reflection signals for speed-of-sound measurements.

The model was used to predict the beam profile for each of the commercially available ultrasonic transducers. For example, Figure 3.4 shows the beam profile for a 1-in. diameter circular-face transducer at $500 \mathrm{kHz}$. In this figure, amplitude intensity is shown by different gray levels in front of the transducer. The transducer face is in the $x-y$ plane at $z=0$, and the axisymmetrical ultrasonic beam propagates along the $\mathrm{z}$-axis.

In this example, the ultrasonic beam is relatively well collimated along the axis out to a distance of about 4 in. from the transducer. The collimated beam has the same diameter as the active face of the transducer. Noticeable diffraction effects begin to occur at about four in., causing the beam to spread away from the axis. Smaller diameter transducers will have smaller collimated beams and shorter near-field/far-field boundaries and the diffraction will cause the radiation to spread at more divergent angles.

When a highly reflective planar surface is placed perpendicular to the beam axis, the ultrasonic radiation returning to the transducer can be envisioned by folding the beam back onto itself at the reflector plane. Thus, if a planar reflector were placed at a distance of, say, 2.5 in. from the transducer in Figure 3.4, the beam cross-section at 5 in. would illuminate the transducer. Such beam folding is valid since the wavefront in the near field cross-section is essentially planar and will be accurately mirrored back to the transducer. By using this radiation pattern example as a guide, a test chamber should be designed to have an internal diameter of about 1.5 to 2 times the transducer face diameter and the reflecting targets should be located at a distance of about 2 to 3 times the transducer active face and oriented parallel to the transducer face (perpendicular to the radiation axis). 


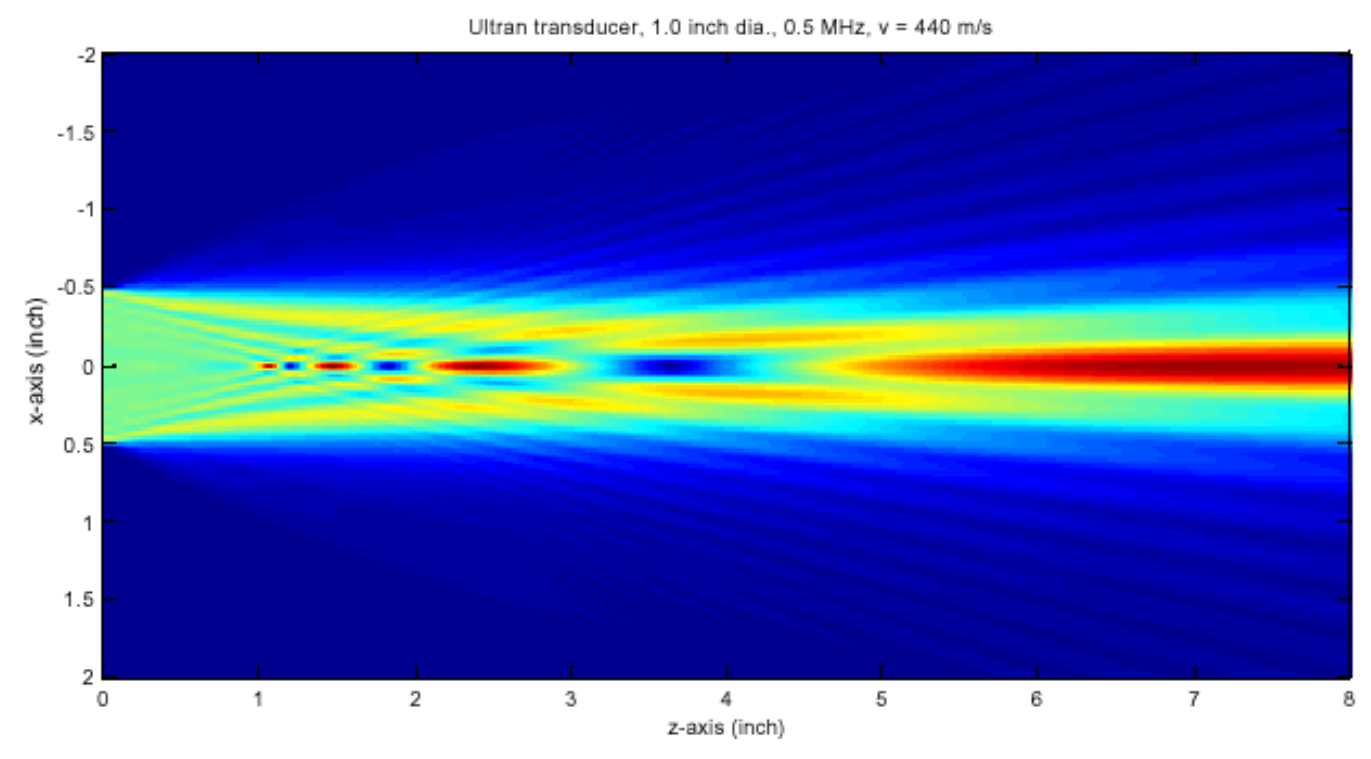

Figure 3.4. Ultrasonic beam profile of 1.0-in. diameter transducer at $500 \mathrm{kHz}$. The near-field/far-field boundary occurs approximately $7 \mathrm{in}$. from the transducer. [Darker gray levels on transducer axis $(x=0)$ are highest amplitudes.]

For the case where two semicircular reflectors are placed in the near field radiation beam, the reflection phenomena described above will occur except that only one half of the total collimated beam energy will be returned from each reflector. Thus, as a consequence, the tworeflector design will split the beam into two equal-area parts and the reflected signal energy will be reduced by $3 \mathrm{~dB}$ relative to that returned from a full cross-section reflector. Minor edge diffraction effects will occur at the dividing line separating the two reflectors but this is not anticipated to interfere with the detected signals.

The effects of viscous attenuation of the ultrasonic pressure waves in the gaseous medium introduce a distance-dependent reduction of the sound amplitude along the beam axis. Since viscous attenuation increases with the square of frequency, the higher frequencies in the ultrasonic pulse spectrum will be more highly absorbed than the lower frequencies, causing noticeable changes in the pulse shape of broadband pulses. For this reason, the two reflecting surfaces should be spaced as close together as practical without causing time-overlapping interference between the reflected waves. The frequency-dependent attenuation will also have second-order effects on the near-field beam collimation, causing an increase in diffraction spreading.

\subsubsection{Near-Field Diffraction and Phase Error}

The ultrasonic transducers used in this application are circular pistons vibrating uniformly in the axial direction normal to their face. At operating frequencies in the range of $250-500 \mathrm{kHz}$ in natural gas, a 1-in. diameter transducer has an equivalent diameter in the range of 16-32 wavelengths. Radiation from different points on this surface results in interference (diffraction effects) and phase errors (time delay errors) at field points in the zone corresponding to the projection of the circular face along the transducer axis out to a distance of about ten 
diameters. When the reflection surfaces in the speed-of-sound test chamber are within this range, possible errors in the effective time of flight of the ultrasonic pulses can occur. In the present speed-of-sound measurements, since the two-way pulse reflection path lengths are in the range of 4-6 in., the possible near-field diffraction errors are of concern.

The ultrasonic particle displacement, $\mathrm{u}(0, \mathrm{z})$, on the axis of a planar piston transducer is derived from the Rayleigh-Sommerfeld integral stating the displacement scalar potential at any point, $z$, on the axis as

$$
\phi(0, z)=-u_{0} \int_{0}^{a} \frac{e^{-j k R}}{R} r d r
$$

When Equation (3.10) is solved for the piston geometry, the sound wave particle displacement is

$$
u(0, z)=\frac{d \phi(0, z)}{d z}=u_{0}\left[e^{-j k z}-\frac{z}{\sqrt{a^{2}+z^{2}}} e^{-j k \sqrt{a^{2}+z^{2}}}\right]
$$

where:

$$
\begin{aligned}
\phi= & \text { displacement scalar potential } \\
z= & \text { axial coordinate } \\
u_{0}= & \text { displacement at the transducer face (uniform excitation) } \\
k= & \omega / v_{\mathrm{g}}=\text { wave number (lossless medium) } \\
R= & {\left[x^{2}+r^{2}\right]^{1 / 2}=\text { ray path from elemental source point on transducer } } \\
& \text { to any point on axis } \\
r= & \text { radial distance from axis to elemental point on transducer face } \\
a= & \text { radius of transducer } \\
\omega= & 2 \pi f=\text { angular frequency } \\
v_{g}= & \text { speed of sound in test gas }
\end{aligned}
$$

Figure 3.5 shows the amplitude interference effects in the axial plane of 1-in. diameter transducers operating at $250 \mathrm{kHz}$ and $500 \mathrm{kHz}$, respectively. These plots are for steady-state single-frequency operation to show the rapid interference variations that can occur in the ultrasonic wave amplitude at distances close to the transducer. In both figures the last broad peak represents the boundary between the near field and the far field along the x-axis, beyond which the amplitude decays inversely with distance. In general, this boundary distance is defined in terms of the transducer parameters as $\mathrm{a}^{2} \mathrm{f} / \mathrm{v}_{\mathrm{g}}$. The near-field boundary distance for the 1 -in. diameter transducer operating at $500 \mathrm{kHz}$ is noted to be four times greater than the boundary for the $250-\mathrm{kHz}$ transducer. Markers on each figure show the positions corresponding to reflections from rigid reflectors located at 2 in. and 3 in. away from the transducer face, i.e., at two-way distances of 4 and 6 inches. 


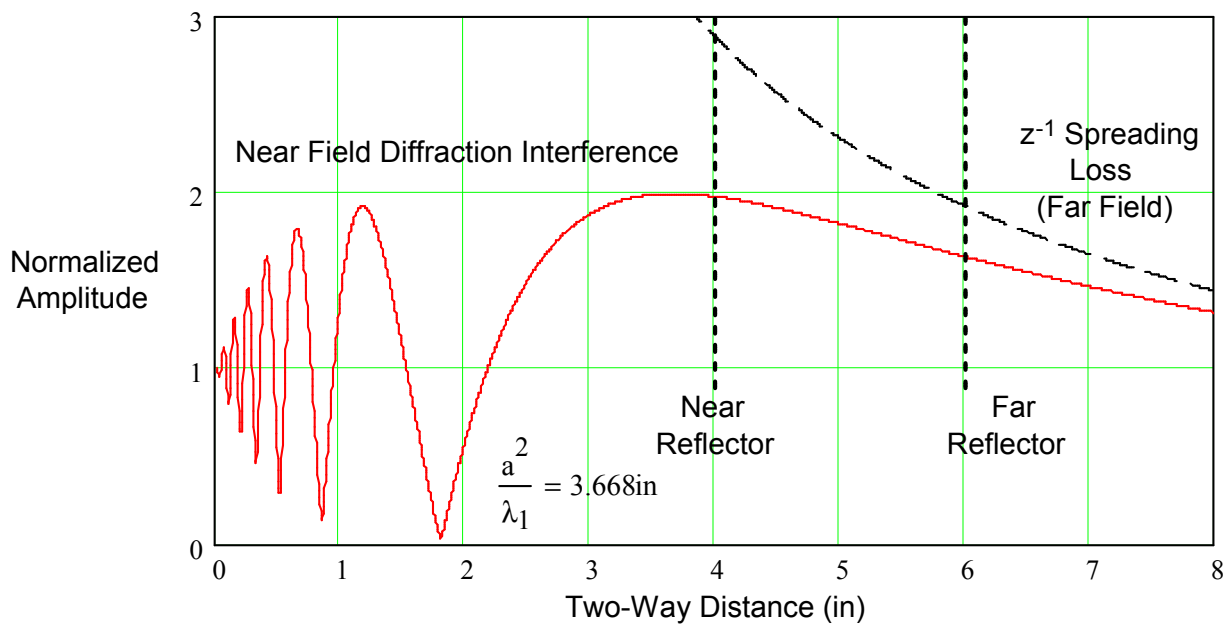

(a) Frequency: $250 \mathrm{kHz} ; v_{\mathrm{g}}=1,420 \mathrm{ft} / \mathrm{sec}$

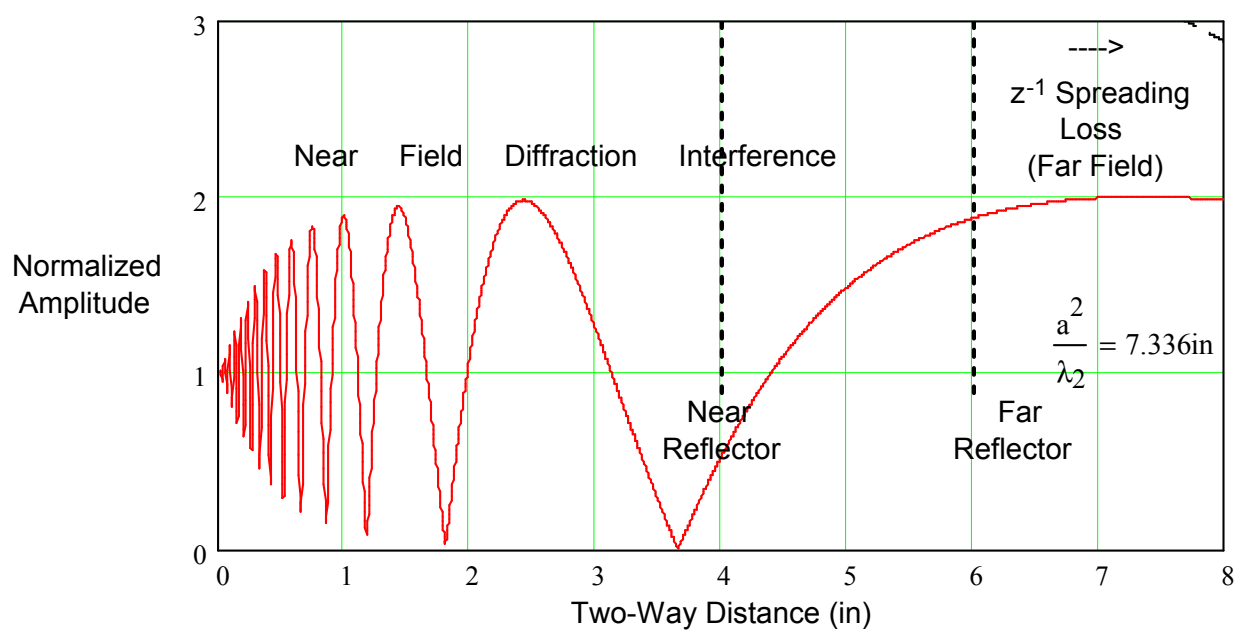

(b) Frequency: $500 \mathrm{kHz} ; \mathrm{v}_{\mathrm{g}}=1,420 \mathrm{ft} / \mathrm{sec}$

Figure 3.5. Near-field on-axis diffraction interference for 1 -in. diameter ultrasonic transducer.

The received reflection signals at the transducer face can be determined approximately by expressing the ultrasonic wave particle displacement at any two-way distance point $(\mathrm{r}, \mathrm{z})$ about the transducer axis and integrating the result over the face of the transducer acting as a receiver. The off-axis wave displacement may be found by applying a two-dimensional spatial transform to the Rayleigh-Sommerfeld equation in Equation (3.10) in the plane normal to the transducer axis at any position along the transducer axis. That is, for the axisymmetric geometry of the transducer, the amplitude at any point on the z-axis and at any radial distance, $r$, away from the axis is 


$$
u(r, z)=u_{0} a \int_{0}^{a} J_{1}(\alpha a) J_{0}(\alpha r) e^{-j \sqrt{k_{r}^{2}-\alpha^{2}}} z
$$

where

$$
\begin{aligned}
\mathrm{a} & =\text { axial spatial frequency } \\
\mathrm{k}_{\mathrm{r}} & =\text { radial wave number } \\
\mathrm{J}_{0}, \mathrm{~J}_{1} & =\text { Bessel functions of zero and first order }
\end{aligned}
$$

From this result, the displacement amplitude integrated over a circle of radius, 2a, corresponding to the receiving face of the transducer, is

$$
\bar{u}(r, z)=2 u_{0} \int_{0}^{\infty} \frac{J_{1}^{2}(\alpha a)}{\alpha} e^{-j k \sqrt{a^{2}+z^{2}}} d \alpha
$$

Figure 3.6 shows the axisymmetrical amplitude distribution along any radial diameter as calculated using Equation (3.12) in planes transverse to the transducer axis at several positions in the near field for the $250 \mathrm{kHz}$ transducer. These plots represent off-axis wave amplitude variations showing that the diffraction effects are distributed in three dimensions but are confined to a cylindrical zone having approximately the same diameter as the transducer. The results are similar for the $500-\mathrm{kHz}$ transducer except that the near-field interference effects extend beyond the indicated reflector positions.

The wave amplitude integrated over the face of the transducer expressed by Equation (3.13) is proportional to the output voltage generated by the transducer acting as a receiver. Figure 3.7 shows the transducer output signal amplitudes received from reflectors located at 2 in. and $3 \mathrm{in}$. away from the transducer face. These signals are complex quantities in which the total cumulative phase of each reflected wave corresponds to the total two-way propagation time delay from the transducer to the reflector and back. The axial wave number, k, divided by the total phase delay at any two-way distance, $2 \mathrm{z}$, is the apparent speed of sound for reflections at that point. By subtracting the speed of sound at the near reflector two-way path length from that of the far reflector, the speed-of-sound error for signals received from the two reflectors may be obtained.

Figure 3.8 shows the speed-of-sound error as a function of the two-way reflection distance for transducers operating at $250 \mathrm{kHz}$ and $500 \mathrm{kHz}$. As an example of the impact of these errors, at $250 \mathrm{kHz}$ in a $1,420-\mathrm{ft} / \mathrm{sec}$ gas typical of a methane-dominant gas at relatively low pressure, the round trip error in apparent speed of sound for either reflector is about $1 \mathrm{ft} / \mathrm{sec}$. However, the difference in the two values for the near and far reflectors is only $0.03 \mathrm{ft} / \mathrm{sec}$. At $500 \mathrm{kHz}$, Figure 3.8 shows that the difference in speed of sound is $0.44 \mathrm{ft} / \mathrm{sec}$ at the same reflector distances. 

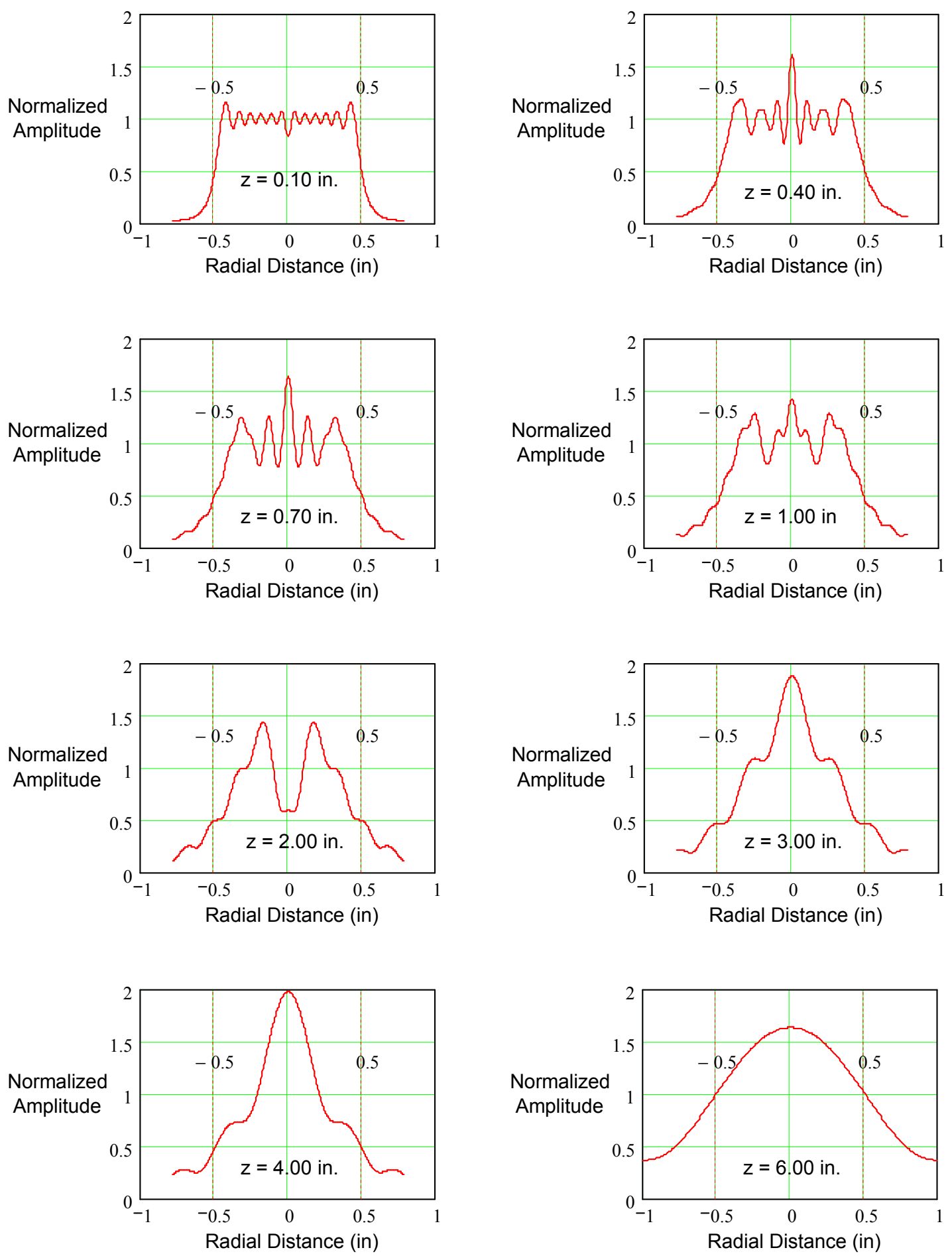

Figure 3.6. Axisymmetrical near-field amplitude distribution about transducer axis. 


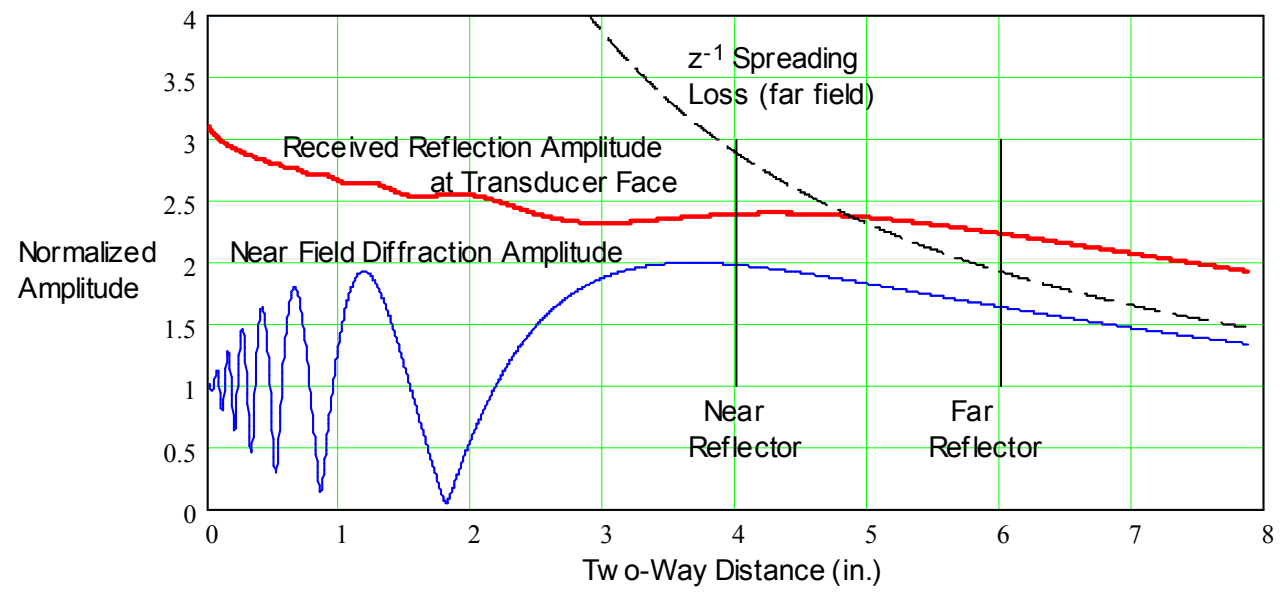

(a) Frequency: $250 \mathrm{kHz}$

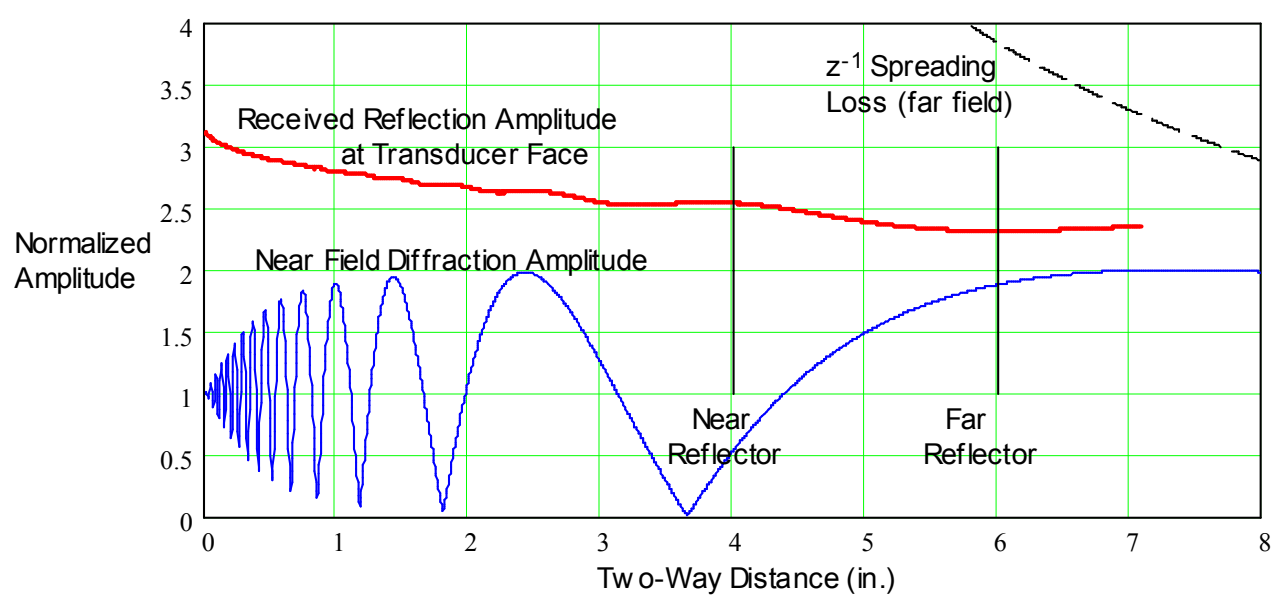

(b) Frequency: $500 \mathrm{kHz}$

Figure 3.7. Combined output signal amplitude from both reflectors. 


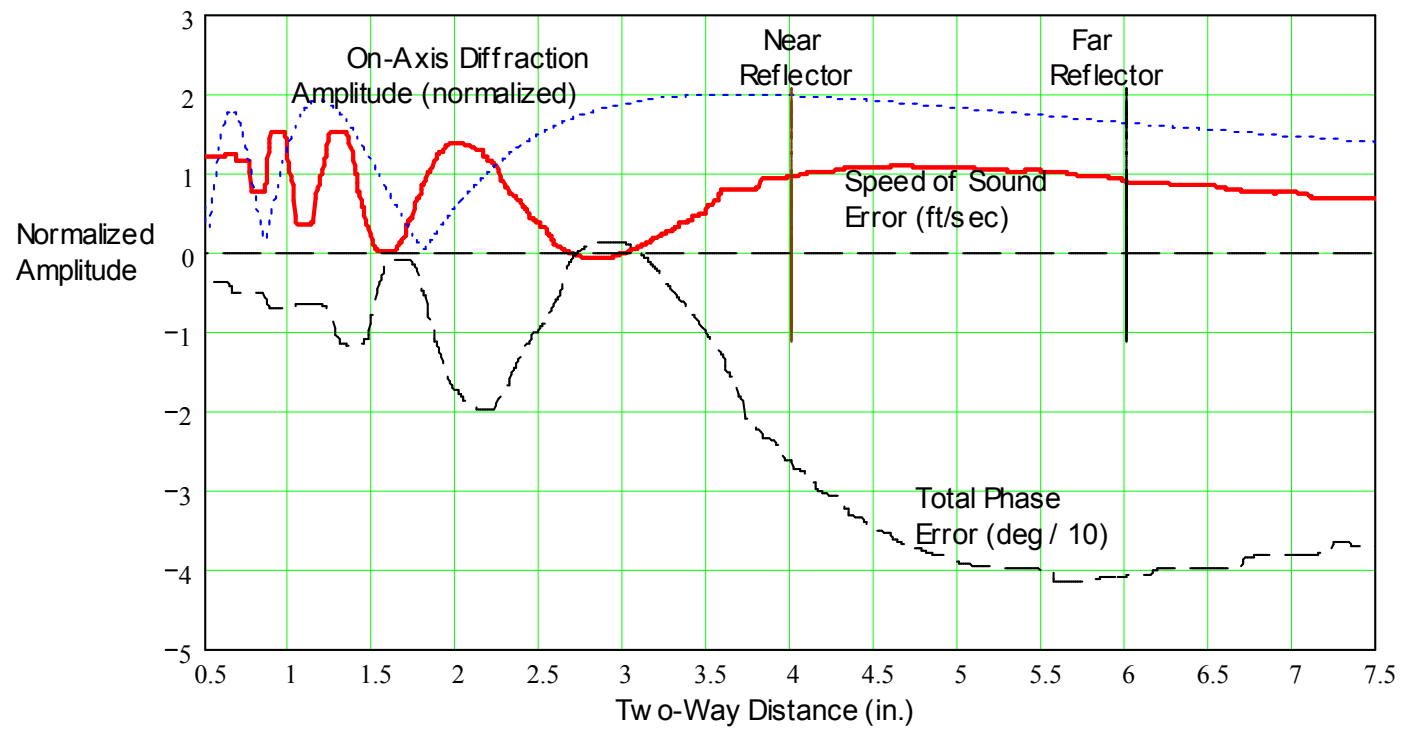

(a) Frequency: $250 \mathrm{kHz}$

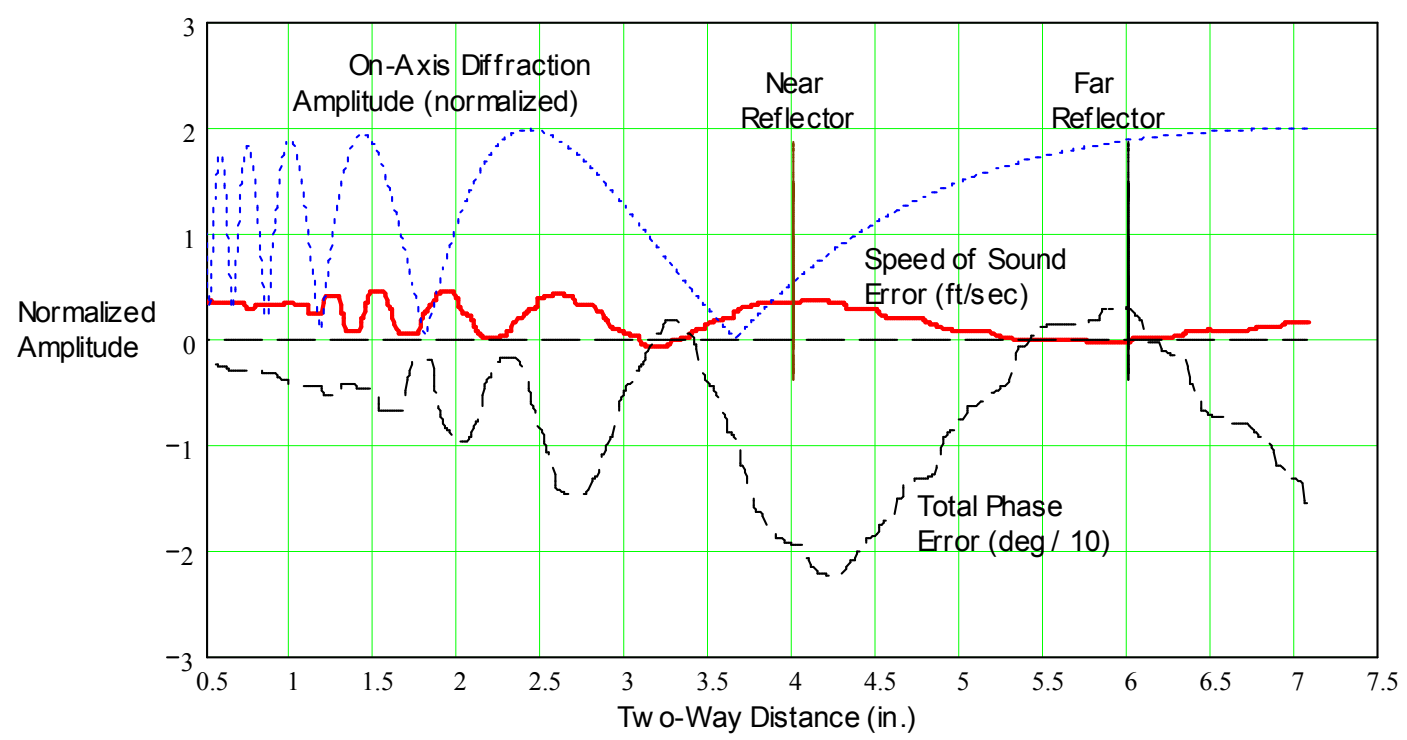

(b) Frequency: $500 \mathrm{kHz}$

Figure 3.8. Near-field diffraction phase delay and speed-of-sound errors vs. two-way reflection distance.

Overall, these results show that the signals received from each reflector are relatively constant in amplitude at $250 \mathrm{kHz}$ and $500 \mathrm{kHz}$ over the near field range of about one to eight diameters away from the transducer face. A small phase-lead effect in the received signals translates into a corresponding increase in speed of sound of about $1 \mathrm{ft} / \mathrm{sec}$ at $250 \mathrm{kHz}$ and about $0.5 \mathrm{ft} / \mathrm{sec}$ at $500 \mathrm{kHz}$, within the limitation of the steady-state single-frequency analysis applied above. However, the positions along the transducer axis at which the variations in apparent speed of sound occur are different for the two frequencies. 
In practical application, the ultrasonic signals used to measure the reflection times of flight are designed to be short-duration sinusoidal pulses having a wide spectral bandwidth. In this case, there will be many interference patterns similar to those shown in Figures 3.5 and 3.6 present in the near field with each one having different positions of peaks and nulls. Thus, the superposition of this spectrum of diffraction patterns will tend to smooth out the amplitude variations in the near field. However, the envelope of the integrated pulses received at the transducer face will be stretched somewhat because of the different geometrical ray path lengths, and the effective total phase delay in the received reflections will remain distorted at values comparable to those found in the steady-state estimates derived above. In general, the steadystate phase error effect on the derived speed of sound may be considered as the worst-case condition. Even so, for 1-in. diameter transducers operating at frequencies in the range of 250$500 \mathrm{kHz}$ with reflectors spaced at less than $3 \mathrm{in}$. away from the transducer face, the worst-case phase error is relatively small. Furthermore, for a particular ultrasonic transducer and operating frequency in a fixed test chamber design, this error in apparent speed of sound will be essentially constant and can be compensated in the chamber calibration process.

\subsubsection{Attenuation and Dispersion}

Molecular relaxation effects in gases cause anomalous absorption of sound energy and attendant changes in the speed of sound that depend on the sound wave frequency. The molecular relaxation frequencies at which a gas absorbs sound energy depend on the particular vibrational and translational resonance modes in the constituent gas molecules and are excited by molecular collisions when sound waves at or near these modal resonance frequencies are present. Energy transfer between molecular collisions and internal molecular vibrations results in irreversible energy losses and, hence, sound attenuation along the path of propagation. Because the gas molecules do not return all of the sound wave energy when resonances occur, the effective specific heat of the gas is modified slightly, resulting in an increase in speed of sound. In polyatomic gases, the molecular resonances are more numerous, and in gas mixtures, the relaxation frequencies of the different species are often close together and may be coupled as a result of collisions between the different species. These molecular-level complexities are dependent on the gas constituents and their concentrations as well as on the gas pressure and temperature. Analytical predictions of the relaxation frequencies and the various inter-molecular coupling effects are practical only for simple gas molecules and only in limited binary and ternary mixtures [Herzfeld and Litovitz (1959); Dain and Lueptow (2001a, 2001b)]. Thus, anomalous attenuation and velocity dispersion in multi-component gas mixtures such as natural gas are properties of the gas that are amenable only to experimental characterization.

The recent work of Dain and Lueptow [2001a], aimed at analytically characterizing certain ternary methane-dominated gas mixtures with $\mathrm{CO}_{2}$ and water vapor diluents, provides some useful insights into the molecular relaxation effects that may be representative of natural gas mixtures. For methane content in the range of 90-95 mol\%, the coupling effects between methane and the diluent gases may be neglected in a first-order model to allow the mixture to be represented as an apparent single-relaxation-mode gas. For this simplified representation, the dimensionless attenuation and dispersion relationships [Herzfeld and Litovitz (1959)] are 


$$
\begin{aligned}
& \frac{v_{g}}{v_{g 0}}=\left[\frac{1+\left(\frac{p_{0}}{p} \omega\right)^{2} \tau_{r}^{2}}{1-(1-A)\left(\frac{p_{0}}{p} \omega\right)^{2} \tau_{r}^{2}}\right]^{\frac{1}{2}} \\
& a_{m r} \lambda=p A\left[\frac{\left(\frac{p_{0}}{p} \omega\right) \tau_{r}}{1-(1-A)\left(\frac{p_{0}}{p} \omega\right) \tau_{r}^{2}}\right]
\end{aligned}
$$

where

$$
\begin{aligned}
\alpha_{\mathrm{mr}} & =\text { molecular relaxation absorption coefficient }\left(\mathrm{m}^{-1}\right) \\
\lambda & =v_{\mathrm{g}} / \mathrm{f}=\text { wavelength }(\mathrm{m}) \\
\omega & =2 \pi \mathrm{f}=\text { angular frequency }(\mathrm{rad} / \mathrm{sec}) \\
\tau_{\mathrm{r}} & =1 / 2 \pi \mathrm{f}_{\mathrm{r}}=\text { relaxation time constant }(\mathrm{sec}) \\
\mathrm{f}_{\mathrm{r}} & =\text { relaxation frequency }(\mathrm{Hz}) \\
\mathrm{p} & =\text { pressure }(\text { psia }) \\
\mathrm{p}_{0} & =\text { reference pressure }(1 \mathrm{~atm} ; 14.73 \mathrm{psia}) \\
\mathrm{A} & =\left(v_{\mathrm{g} \infty}{ }^{2}-v_{\mathrm{g}}^{2}\right) / v_{\mathrm{g} \infty}^{2} \\
v_{\mathrm{g} 0} & =\text { speed of sound at zero frequency } \\
v_{\mathrm{gas}} & =\text { speed of sound for } \mathrm{f}>>\mathrm{f}_{\mathrm{r}}
\end{aligned}
$$

Gases exhibit additional acoustic attenuation effects at the macroscopic level as a result of viscosity and heat conduction, both of which increase monotonically with the square of frequency. This absorption effect is combined with the molecular relaxation effects to give the total sound attenuation in the gas. The corresponding dimensionless form of this 'classical' attenuation effect, including the explicit dependence on pressure, is expressed as

$$
\alpha_{\text {class }} \lambda=A_{c} f \frac{p}{p_{0}}
$$


where

$$
\begin{aligned}
A_{c} & =\mathrm{F}\left(\eta, \mathrm{k}, \gamma_{0}, \mathrm{C}_{\mathrm{po}}, \mathrm{M}\right)=\text { classical absorption coefficient } \\
\eta & =\text { shear viscosity } \\
\mathrm{k} & =\text { thermal conductivity } \\
\gamma_{0} & =\text { ratio of specific heats } \\
\mathrm{C}_{\mathrm{po}} & =\text { specific heat at constant pressure } \\
\mathrm{M} & =\text { molar mass }
\end{aligned}
$$

The speed of sound in natural gas at very low frequencies (typically two decades below the lowest molecular relaxation frequency) is the same as the value computed using the AGA-8 equation of state. Representative values of the other parameters, $\tau_{\mathrm{r}}$ and $\mathrm{A}$, in Equations (3.14) and (3.15) were extracted from graphic illustrations in Dain and Lueptow [2001a] at a pressure of $1 \mathrm{~atm}$ to yield

$$
\begin{gathered}
\tau_{\mathrm{r}}=9.96 \times 10^{-9} \mathrm{sec} \\
\mathrm{A}=0.0180
\end{gathered}
$$

Similarly, to be consistent with these values, the calculated graphic illustrations of Dain and Lueptow were used to extract the matching classical attenuation factor to yield

$$
\mathrm{A}_{\mathrm{c}}=4.84 \times 10^{-9} \mathrm{sec}^{-1}
$$

Figures 3.9 through 3.12 illustrate these representative anomalous effects of molecular relaxation when applied in a very simplified and approximate way to a natural gas calibration mixture (designated as Natural Gas \#3 in the appendix). The curves in these figures are calculated for a temperature of $80.33^{\circ} \mathrm{F}(300 \mathrm{~K})$ and pressures ranging from $1 \mathrm{~atm}(14.73 \mathrm{psia})$ to $7 \mathrm{~atm}(103.1 \mathrm{psia})$ to specifically illustrate the effects of both sound wave frequency and pressure. For the pressure range shown, the speed of sound decreases with pressure by about $-8 \mathrm{ft} / \mathrm{sec}$ for the low-frequency values, $\mathrm{v}_{\mathrm{go}}$, in the calibration gas mixture. The molecular relaxation effects introduce a step-like increase in the speed of sound beginning at a frequency of about $30-40 \mathrm{kHz}$ at $1 \mathrm{~atm}$ and up to about $200 \mathrm{kHz}$ at $7 \mathrm{~atm}$, rising to final values of speed of sound that are about $12 \mathrm{ft} / \mathrm{sec}$ higher than the low-frequency values (i.e., up by approx. $0.85 \%$ ) over a two-decade frequency range. This simplified methane-dominant model indicates that, for ultrasonic signals at discrete frequencies of 250,500 , and $750 \mathrm{kHz}$, the relaxation effects result in speed-of-sound values that are frequency dependent and higher than the low-frequency AGA-8 values as shown in Table 3.1 . 


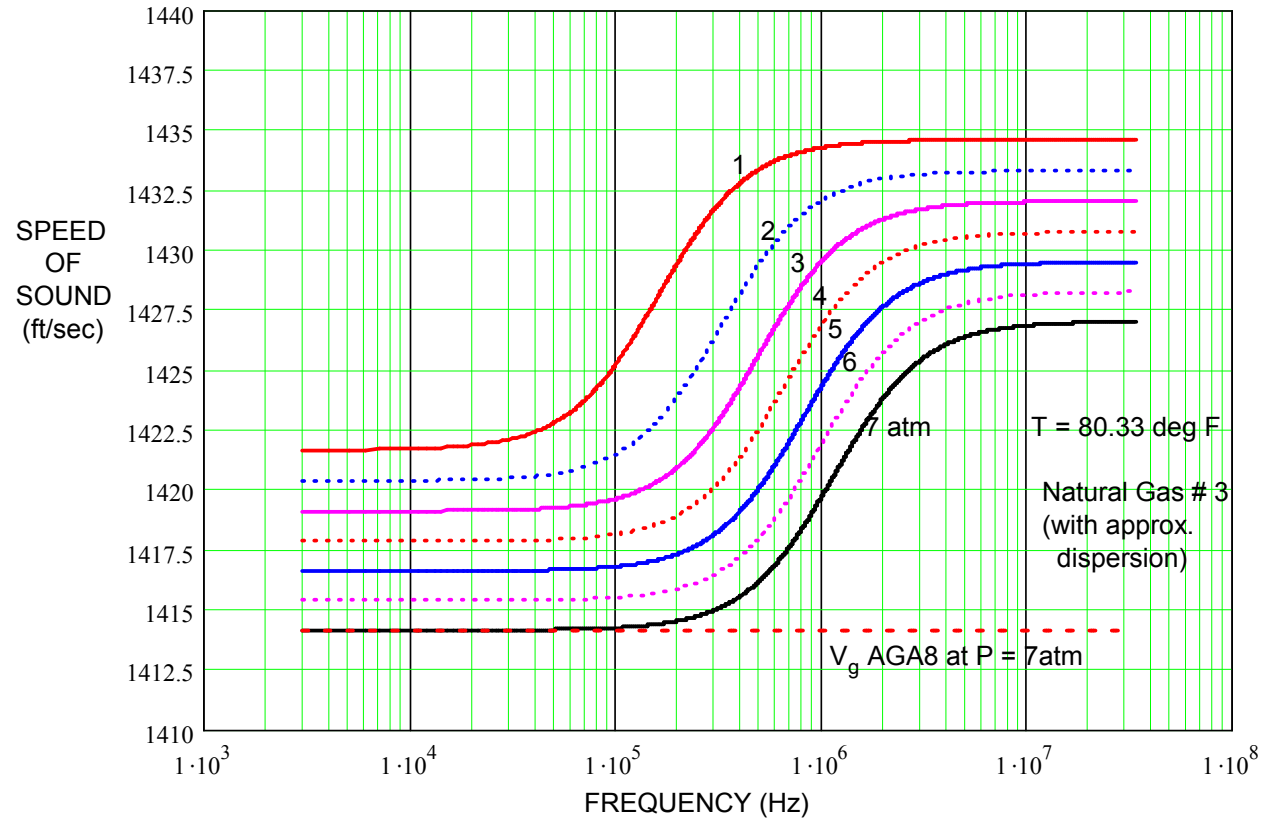

Figure 3.9. Ultrasonic velocity dispersion in Natural Gas \#3 using approximate dispersion parameters in Equation (3.16).

Dimensionless Attenuation $(\alpha \lambda)$

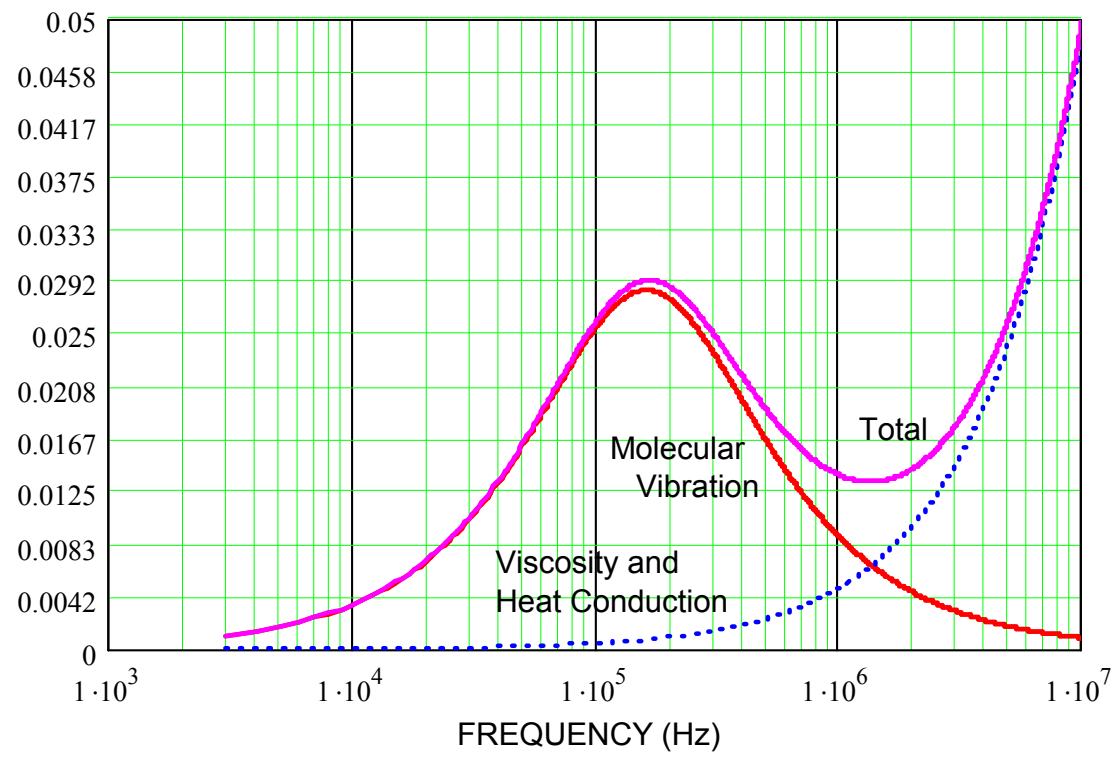

Figure 3.10. Ultrasonic attenuation at $1 \mathrm{~atm}$ in Natural Gas \#3 using approximate dispersion and classical attenuation parameters in Equations (3.16) and (3.17). 


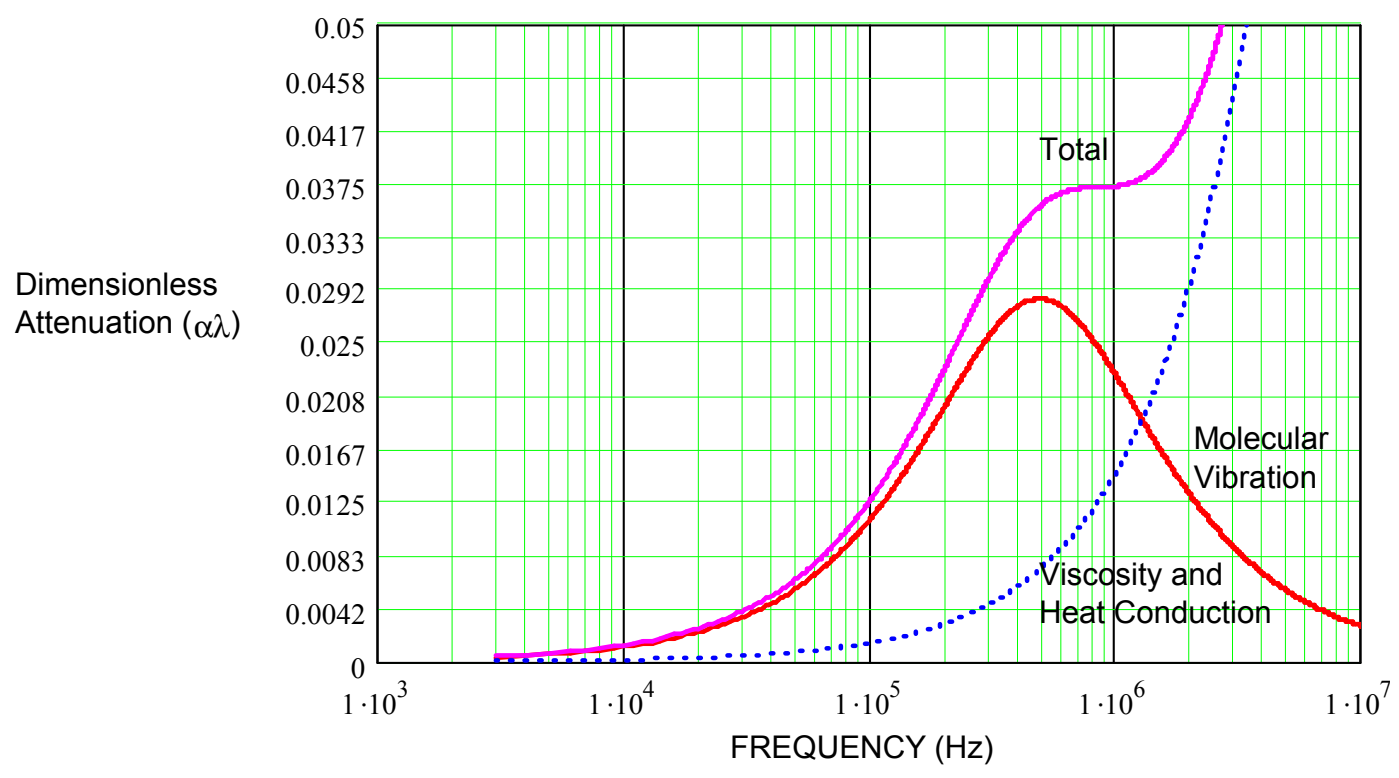

Figure 3.11. Ultrasonic attenuation at 3 atm in Natural Gas \#3 using approximate dispersion and classical attenuation parameters in Equations (3.16) and (3.17).

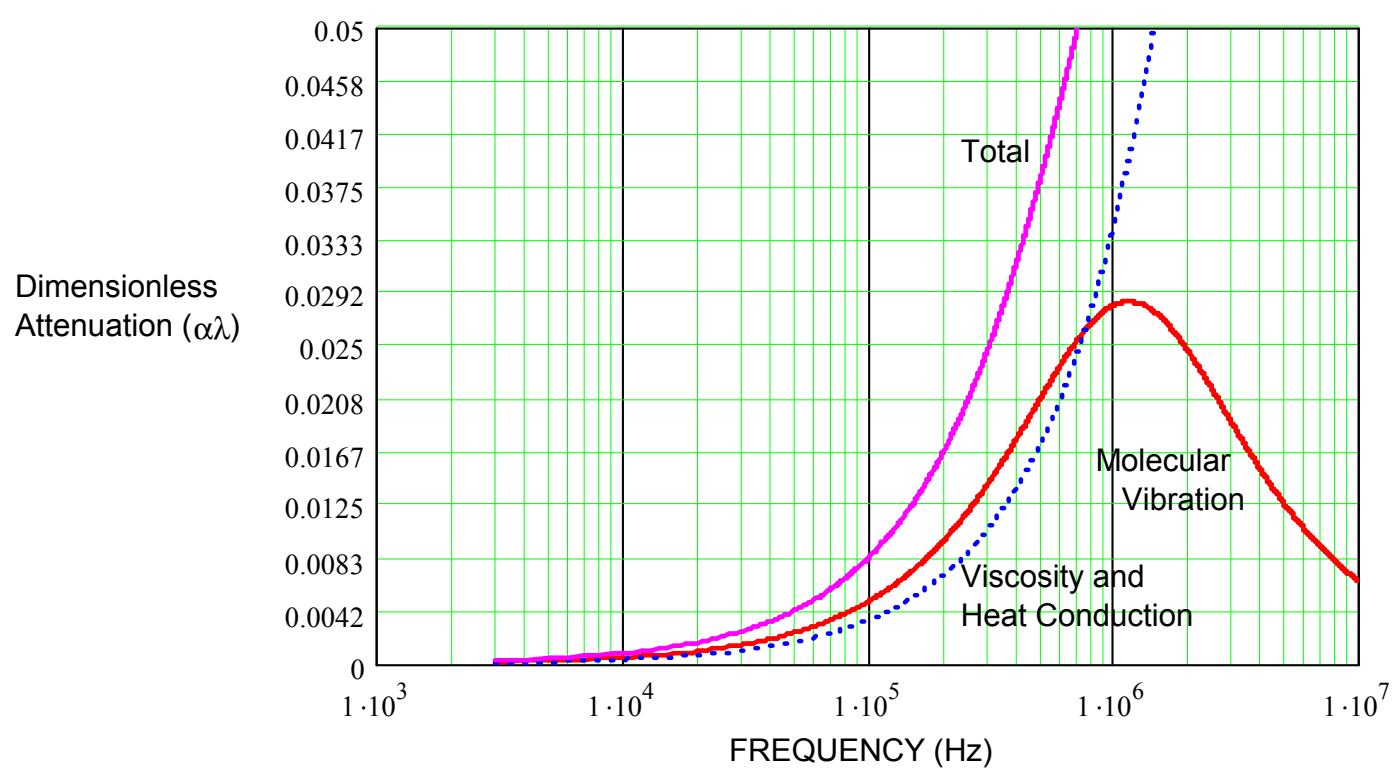

Figure 3.12. Ultrasonic attenuation at 7 atm in Natural Gas \#3 using approximate dispersion and classical attenuation parameters in Equations (3.16) and (3.17). 
Table 3.1. Speed of sound at three ultrasonic frequencies.

\begin{tabular}{|c|c|c|c|c|c|c|c|c|c|}
\hline \multirow{3}{*}{$\begin{array}{c}\text { FREQ } \\
\text { (kHz) }\end{array}$} & \multicolumn{9}{|c|}{ Speed of Sound } \\
\hline & \multicolumn{3}{|c|}{1 atm (14.73 psia) } & \multicolumn{3}{|c|}{$3 \mathrm{~atm}$ (44.19 psia) } & \multicolumn{3}{|c|}{7 atm (103.1 psia) } \\
\hline & $\begin{array}{l}\text { Vgo } \\
\text { (ft/sec) }\end{array}$ & $\begin{array}{c}V g \\
\text { (ft/sec) }\end{array}$ & $\begin{array}{l}\text { Change } \\
\text { (ft/sec) }\end{array}$ & $\begin{array}{c}\text { Vgo } \\
(\mathrm{ft} / \mathrm{sec})\end{array}$ & $\begin{array}{c}\mathrm{Vg} \\
\text { (ft/sec) }\end{array}$ & $\begin{array}{l}\text { Change } \\
\text { (ft/sec) }\end{array}$ & $\begin{array}{c}\text { Vgo } \\
(\mathrm{ft} / \mathrm{sec})\end{array}$ & $\begin{array}{c}\mathrm{Vg} \\
\text { (ft/se }\end{array}$ & $\begin{array}{l}\text { Change } \\
\text { (ft/sec) }\end{array}$ \\
\hline 250 & 1421.7 & 1430.8 & 16.1 & 1419.1 & 1421.8 & 2.7 & 1414.2 & 1414.7 & 0.5 \\
\hline 500 & 1421.7 & 1433.4 & 18.7 & 1419.1 & 1425.7 & 6.6 & 1414.2 & 1416.2 & 2.0 \\
\hline 750 & 1421.7 & 1434.0 & 19.3 & 1419.1 & 1428.2 & 9.1 & 1414.2 & 1418.0 & 3.8 \\
\hline
\end{tabular}

From these results, practical ultrasonic measurements of the desired speed-of-sound (i.e., the low-frequency (AGA-8) value will tend to be high by amounts ranging from about $0.05 \%$ at the lower frequency and higher pressure to about $0.5 \%$ at the higher frequency and lower pressure. An additional examination of these trends has indicated that natural gas having a higher methane content will exhibit a somewhat smaller shift in speed of sound, whereas a lower methane gas will have a somewhat larger shift.

Figures 3.10 through 3.12 show, for the simplified methane-dominant model described above, the dimensionless total attenuation, $\alpha \lambda$ tot, versus frequency, indicating the anomalous attenuation effects of molecular relaxation combined with classical viscous and heat conduction losses. For each of the gas pressures shown, the total attenuation, $\alpha \lambda \operatorname{tot}(\mathrm{f} / \mathrm{vg})$, has been determined for a 5-in. two-way sound propagation path at frequencies of 250,500 , and $750 \mathrm{kHz}$ based on the speed-of-sound values in Table 3.1. The attenuation values are listed in Table 3.2, giving the two-way transmission loss in decibels relative to the initially transmitted signal strength at the transducer face.

Table 3.2. Total attenuation of ultrasonic waves in methane-dominant gas for three frequencies and pressures.

\begin{tabular}{|c|c|c|c|}
\hline \multirow{2}{*}{$\begin{array}{l}\text { FREQ } \\
\text { (kHz) }\end{array}$} & \multicolumn{3}{|c|}{$\begin{array}{l}\text { Relative Sound Wave Amplitude } \\
\text { After Traveling 5-In. Propagation Path Length }\end{array}$} \\
\hline & 1 atm (14.73 psia) & $3 \mathrm{~atm}$ (44.19 psia) & 7 atm (103.1 psia) \\
\hline 250 & $-17.2 \mathrm{~dB}$ & $-17.0 \mathrm{~dB}$ & $-13.1 \mathrm{~dB}$ \\
\hline 500 & $-24.1 \mathrm{~dB}$ & $-45.5 \mathrm{~dB}$ & $-48.8 \mathrm{~dB}$ \\
\hline 750 & $-29.1 \mathrm{~dB}$ & $-70.6 \mathrm{~dB}$ & $-99.7 \mathrm{~dB}$ \\
\hline
\end{tabular}

The attenuation plots at pressures of 3 atm and 7 atm show the upward shift in the molecular vibration attenuation peak and the dominant effect of classical absorption at frequencies above about $750 \mathrm{kHz}$. Although not shown in these figures, the relatively strong attenuation effects at the higher pressures will be mitigated, in part, by the improved acoustic coupling of the transducer as the gas density increases with pressure. This improvement is estimated to add approximately $+10 \mathrm{~dB}$ to the signal strength at $3 \mathrm{~atm}$ and $+17.1 \mathrm{~dB}$ at $7 \mathrm{~atm}$. A general conclusion from this simplified model analysis is that the optimum operating transducer center frequency for measuring the speed of sound in natural gas is in the range of 250 to 500 
$\mathrm{kHz}$, depending on the final sound propagation path length and the operating pressure at which the measurements are made. Frequencies below this range are insufficient for resolving the time of flight of the sound pulses and frequencies above this range will tend to experience excessive attenuation losses resulting in poor signal-to-noise ratio in the received pulse signals.

\subsubsection{Cross-Correlation Signal Analysis}

A cross-correlation procedure was used to find the time difference between the reflected signals from the two targets. This procedure compares the two ultrasonic pulses with respect to their time-domain wave shapes to determine the statistically optimum time delay required to fit the early reflection onto the later reflection. Formally, the cross-correlation of two time domain signals is a measure of the coherence or quantitative similarity of the two waveforms and, since the two reflected pulses are expected to be very similar, the time lag required to indicate the best fit of the two waveforms can be expected to indicate the best statistical estimate of the difference in the transit times, $\Delta \mathrm{t}$, for the two reflections. By applying a time window to bracket and separate the two reflected pulses, the waveforms may then be processed to yield the best-fit time shift. To compute the cross-correlation, a progressively increasing time lag is applied to the first signal, shifting only by one digital sample time interval for each time lag step, and the average product of the two time series at each time lag step is then computed. The average product is a function of the time lag parameter and is a measure of the statistical coherence between the waveforms at each time lag condition. This fact points out the need to accurately resolve the individual reflection waveforms so that the resolution in time lag will be capable of yielding the reflection pulse transit time delay, $\Delta \mathrm{t}$, within the resolution limit required for energy meter speed-of-sound calculations. When the product is at its maximum positive value, the corresponding time lag is used as the value of $\Delta t$ for the pair of reflections being analyzed. To ensure that the resolution of this time lag value is accurate and representative of the true ultrasonic pulse time delay, a predetermined number of reflection waveform pairs are averaged prior to the crosscorrelation process to reduce their incoherent noise content. Additional smoothing of the derived cross-correlation functions may also be applied by averaging a predetermined number of successively calculated cross-correlation functions before selecting the time lag value.

Figure 3.13 shows a simplified diagram of the speed-of-sound system and its signal processing sequence.

The clarity by which the coherence peak of the cross-correlation function may be selected depends upon how sharply the maximum coherence lobe stands out with respect to its neighbors. Short time duration ultrasonic pulses will yield fast-changing correlation functions and, hence, will have better defined coherence peaks than long-duration pulses. Since the frequency spectrum of a short-duration pulse is inherently wider than that of a long-duration pulse, the ultrasonic transducer used in the system must be capable of generating and detecting ultrasonic pulses having the required bandwidth.

Figures 3.14 through 3.16 show three modeled waveforms with different time durations and corresponding bandwidths centered at $300 \mathrm{kHz}$. For simplicity of illustration, the pulse waveforms were computed by multiplying a sine wave by a Gaussian envelope. Each figure shows the waveform, its frequency spectrum, and the autocorrelation function of the waveform. Figure 3.14 shows a pulse waveform having about 20 cycles of oscillation and a corresponding $-6 \mathrm{~dB}$ bandwidth of approximately $40 \mathrm{kHz}(13.3 \%$ of $300 \mathrm{kHz})$. The autocorrelation function 


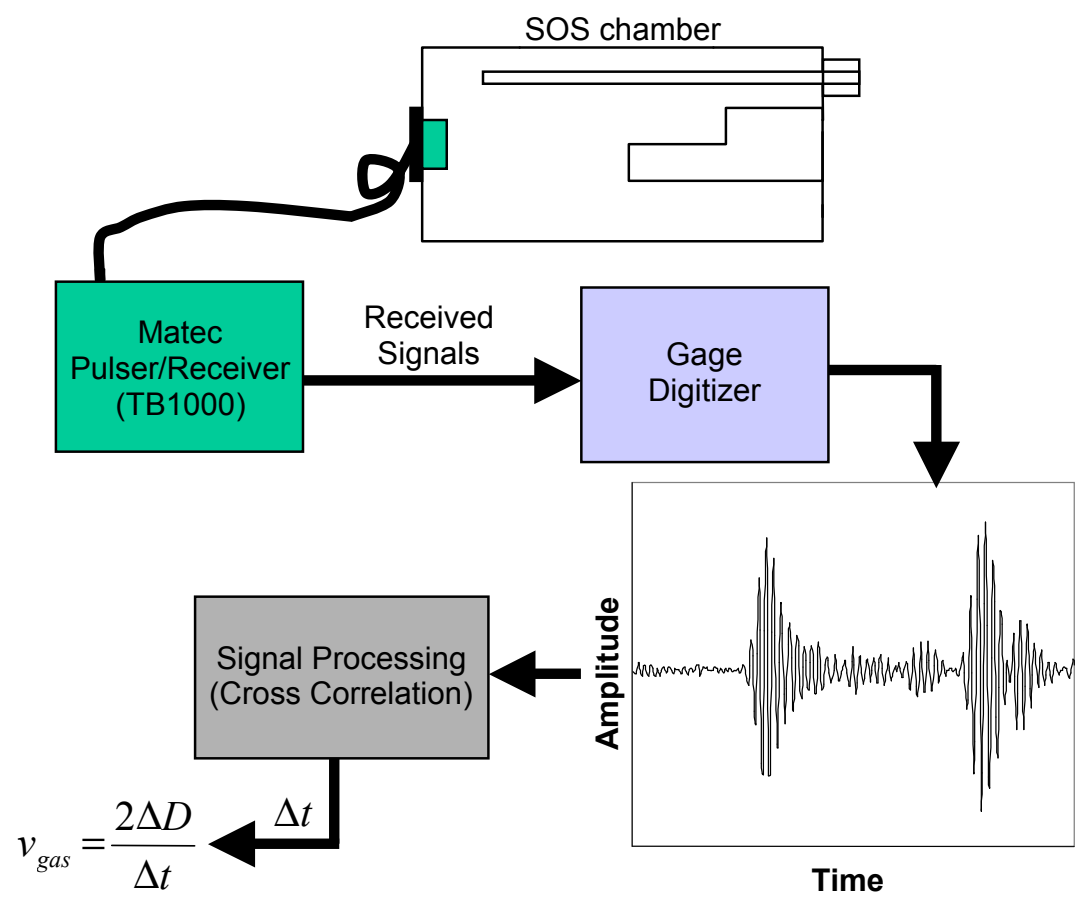

Figure 3.13. Speed-of-sound measurement and signal processing data flow.

for this waveform shows several oscillatory coherence lobes with the central positive lobe being almost indistinguishable in height from its neighbors. Also noted in this figure is the fact that the various positive coherence lobes have a time lag spacing that is identical to the period of the sine wave in the pulse waveform. The waveform shown in Figure 3.15 has a smaller pulse width (7 cycles) and a larger bandwidth $(40 \%$ of $300 \mathrm{kHz})$. In this case, the autocorrelation function has a more distinctive coherence peak that could more easily be selected to yield the optimum time lag value. Figure 3.16 shows a pulse waveform having a very short time duration (2 cycles) and a very wide bandwidth $(100 \%$ of $300 \mathrm{kHz})$. In this ideal case, the autocorrelation function has a sharply defined coherence peak.

The ultrasonic transducer used in the 1999-2000 speed-of-sound measurements had an operating bandwidth of only about $10-15 \%$ of its center frequency. Figures 3.17 and 3.18 show that the reflected waveforms had many cycles and a cross-correlation function without a sharply distinctive peak. In addition, the frequency-dependent attenuation of ultrasound in the natural gas altered the shapes of the reflected waveforms because of the different distances that each reflection must travel. The resulting changes in pulse similarity modify the envelope shape of the cross-correlation function and the values of the coherence peaks. For the example shown, the narrow bandwidth pulses and their dissimilar waveforms erroneously caused a later-occurring coherence lobe to be the maximum value and, hence, to indicate a greater time lag than the proper transit time between the reflected pulses. This time delay error was sufficient to cause an error of $30 \mathrm{ft} / \mathrm{sec}$ in the derived speed of sound. When the indicated speed of sound was reduced by $5 \mu \mathrm{sec}$, corresponding to one period of the $200 \mathrm{kHz}$ pulse center frequency, the error in speed of sound was essentially eliminated. 

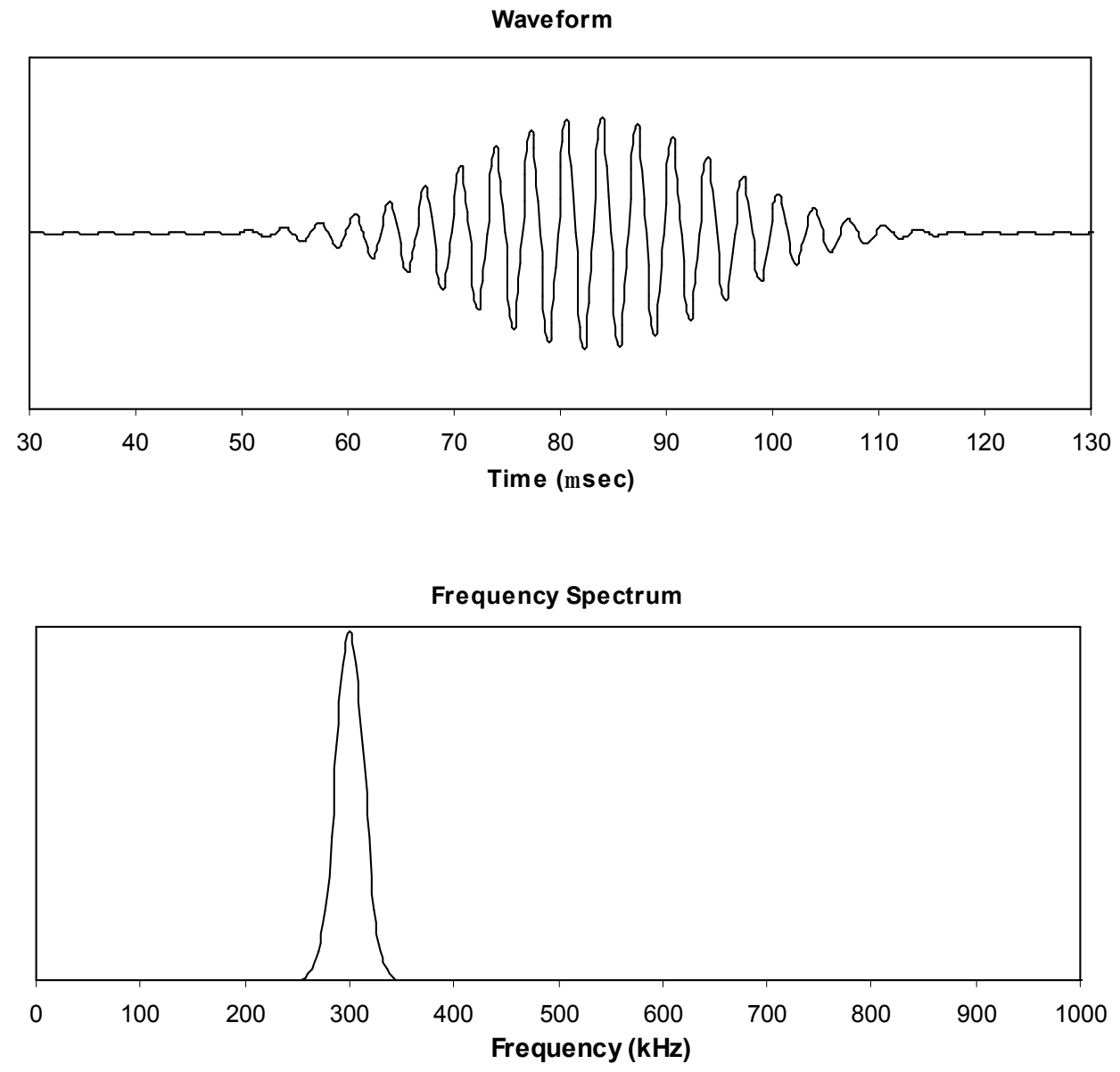

Correlation

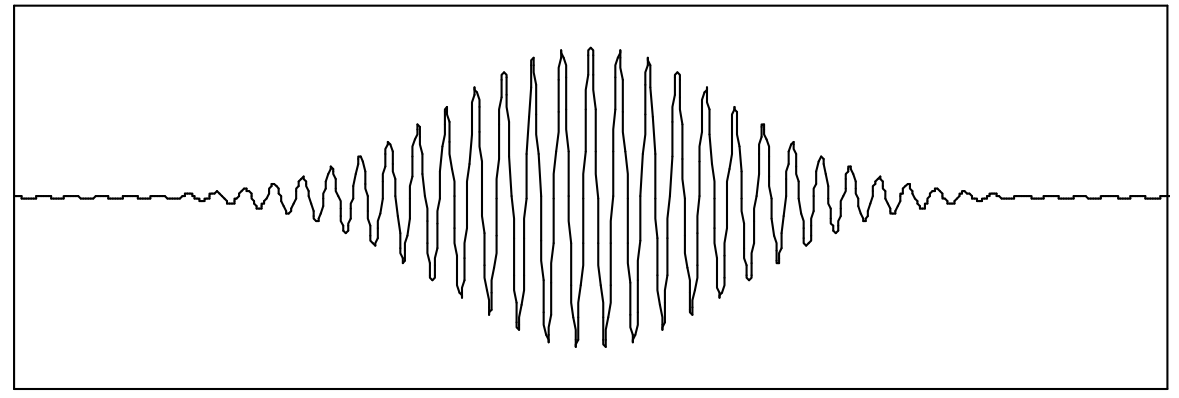

Figure 3.14. Simulated pulse at $300 \mathrm{kHz}$ having $-6 \mathrm{~dB}$ bandwidth of $13.3 \%$ of center frequency. 


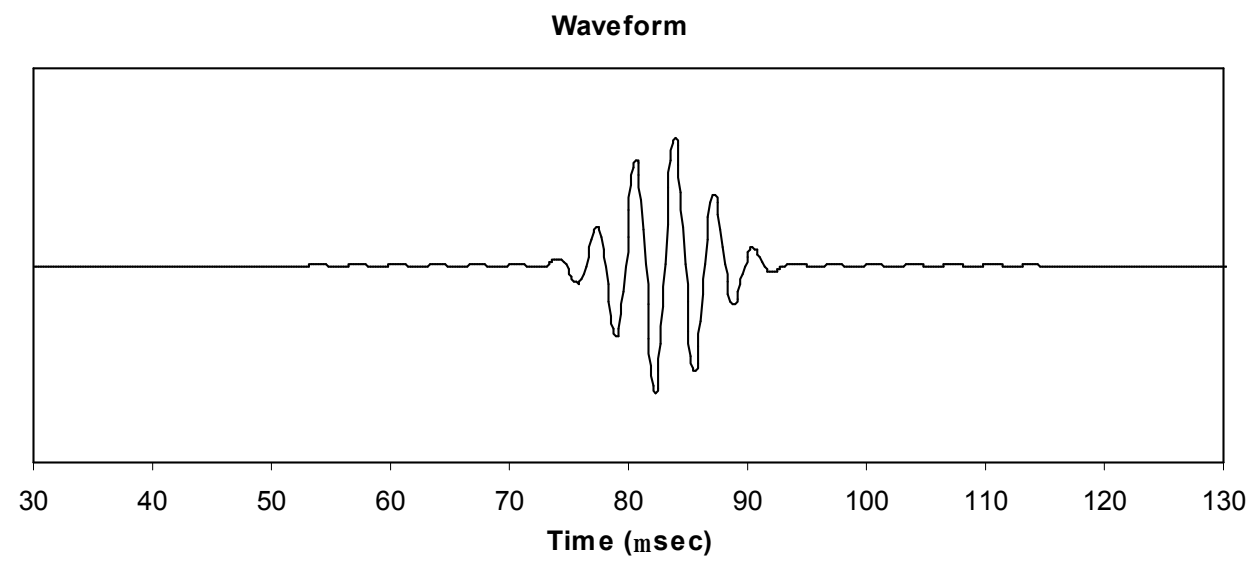

Frequency Spectrum

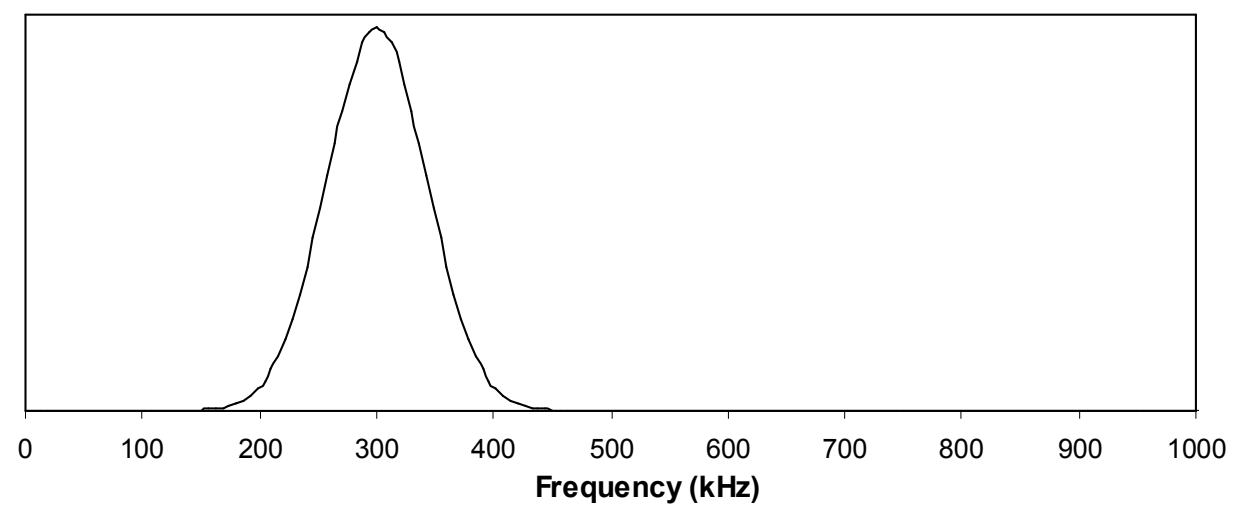

Correlation

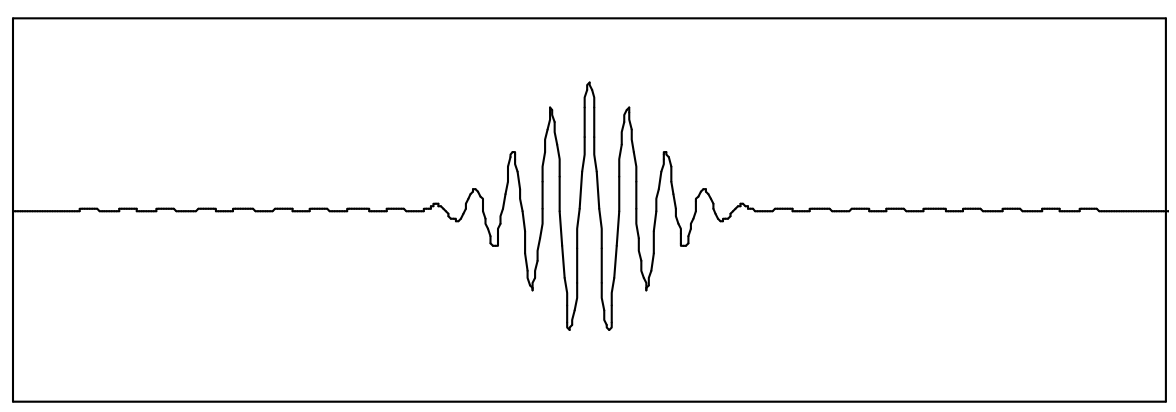

Figure 3.15. Simulated pulse at $300 \mathrm{kHz}$ having $-6 \mathrm{~dB}$ bandwidth of $40 \%$ of center frequency. 


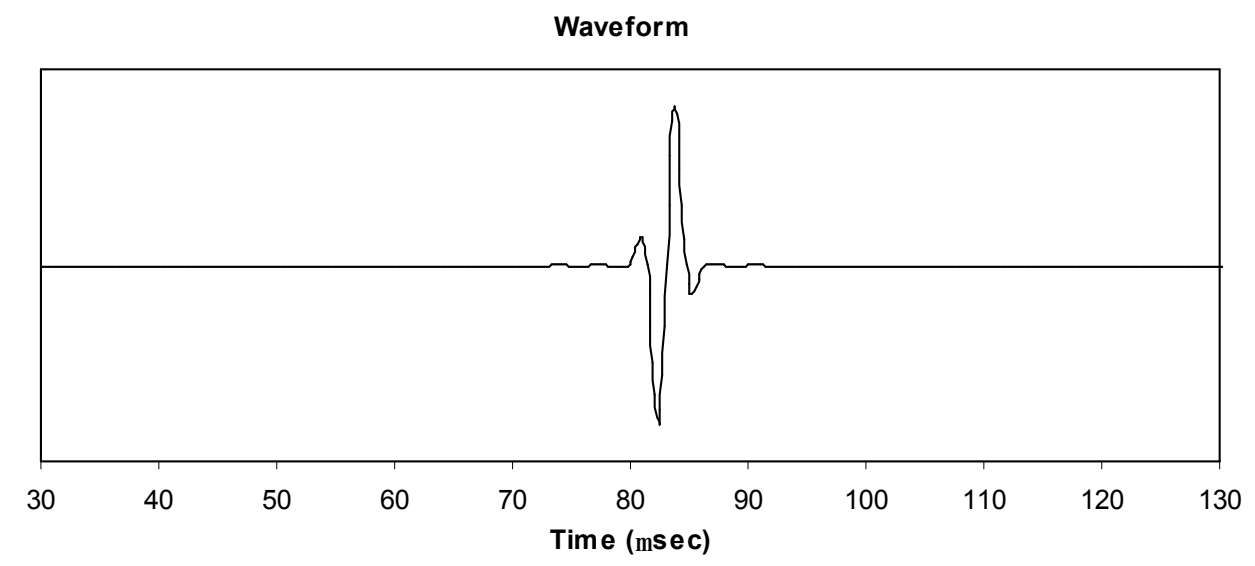

Frequency Spectrum

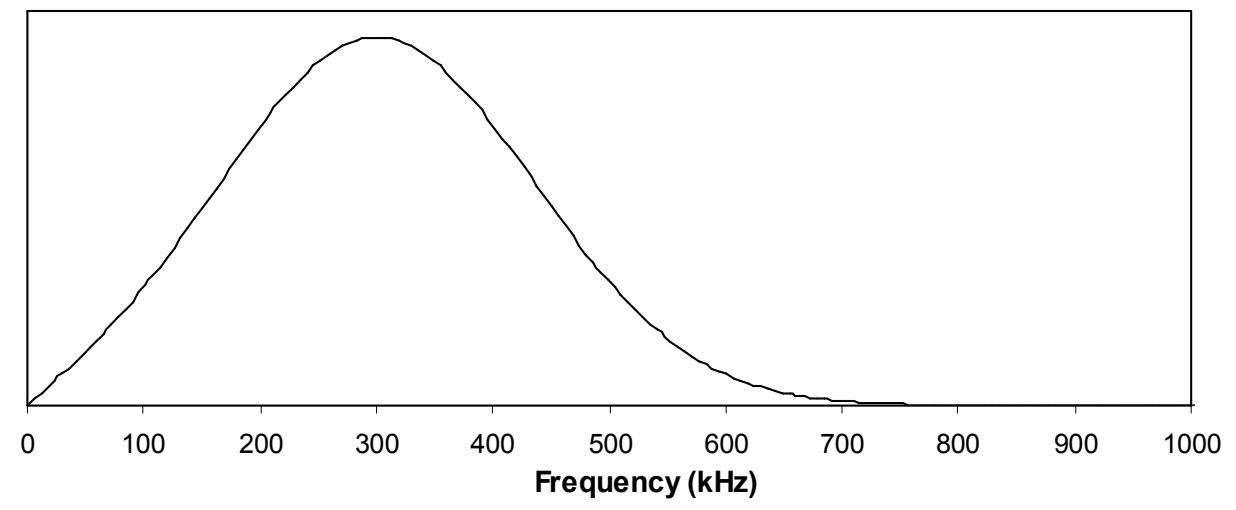

Correlation

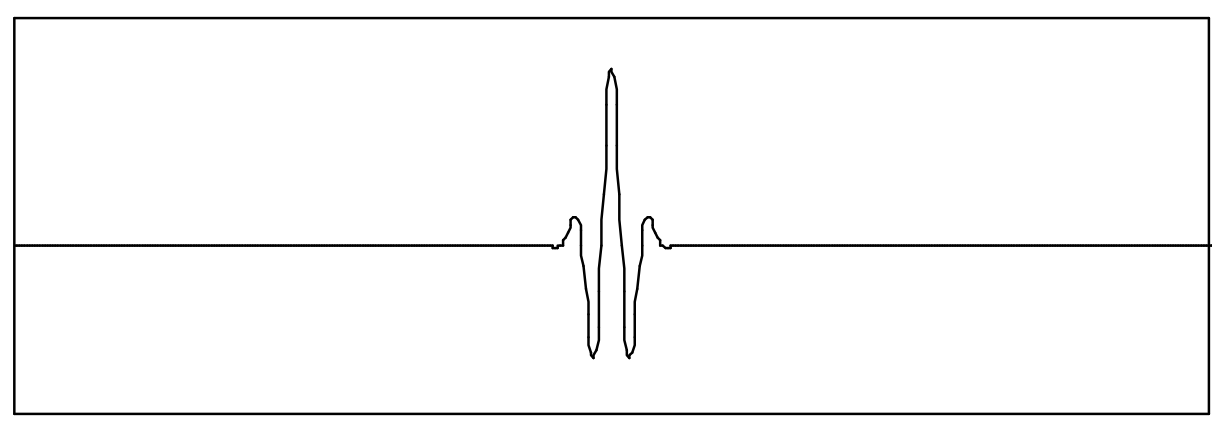

Figure 3.16. Simulated pulse at $300 \mathrm{kHz}$ having $-6 \mathrm{~dB}$ bandwidth of $100 \%$ of center frequency. 


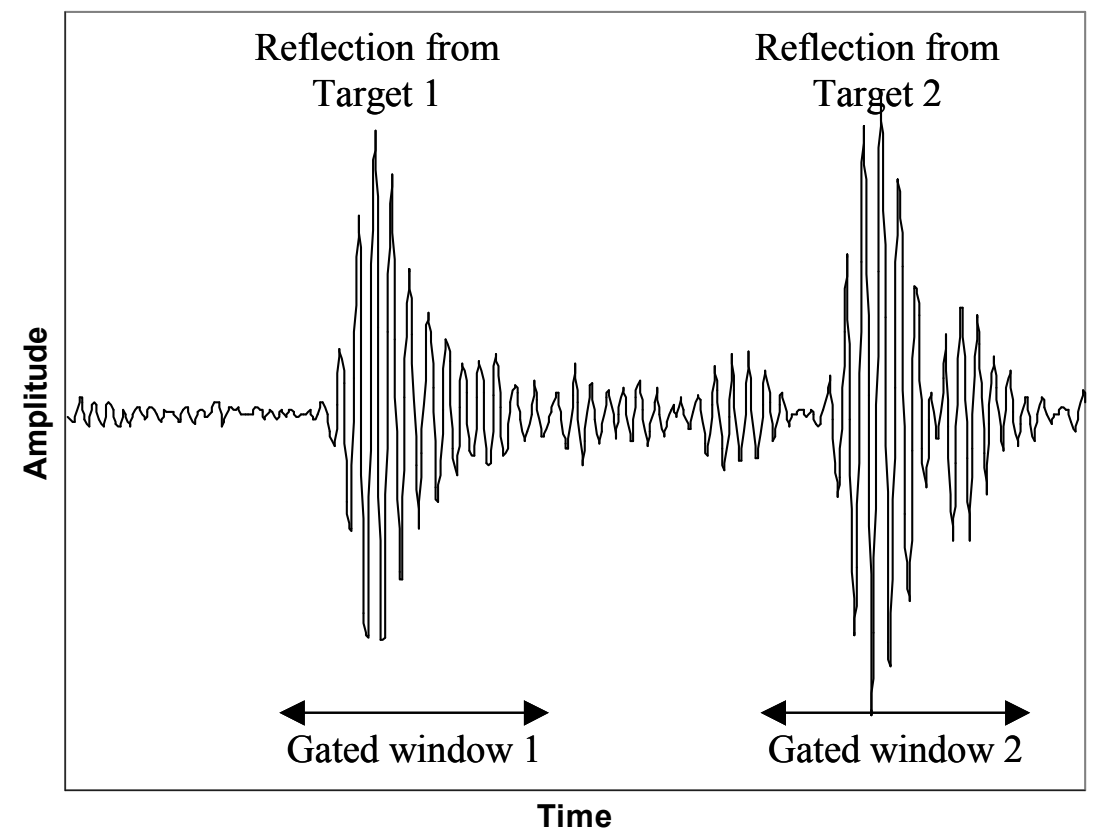

Figure 3.17. Reflected signals from Target 1 and Target 2 in 1999-2000 speedof-sound test chamber. [Transducer resonance frequency: $200 \mathrm{kHz}$;

Bandwidth: $10-15 \%$ of center frequency]

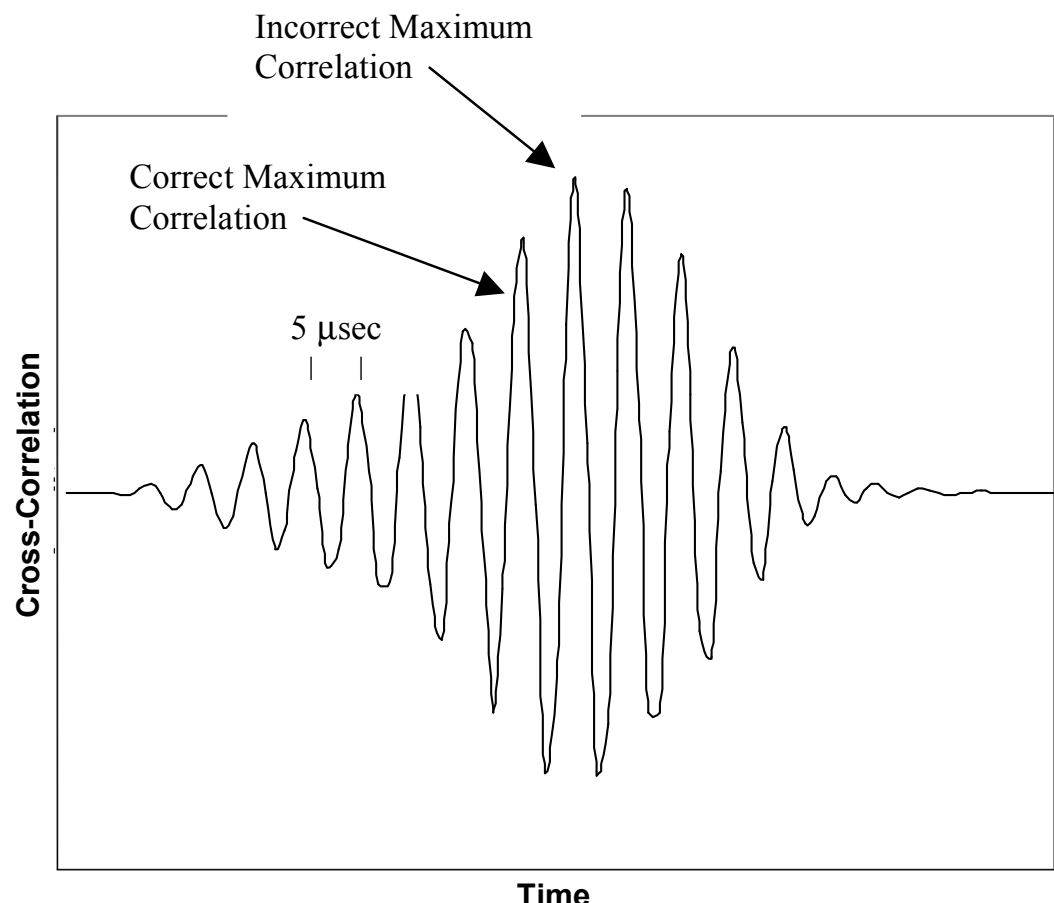

Figure 3.18. Cross-correlation function for reflections from Target 1 and Target 2 in 1999-2000 speed-of-sound test chamber. 


\subsubsection{Speed-of-Sound Sensor Bench Tests}

The time-of-flight speed-of-sound measurements needed in the energy meter design require an ultrasonic transducer capable of gas-coupled operation in the frequency range of $250-500 \mathrm{kHz}$ having high sensitivity and the widest practical bandwidth (typically $50 \%$ of center frequency or wider). Transducers adequately satisfying these characteristics are not commercially available, although experimental prototype devices with specialized performance features were obtained on special order from two manufacturers. These transducers were provided on a 'best effort' basis to meet the desired performance specifications and goals. Three such prototype transducers were evaluated for use in the speed-of-sound sensor. In addition, two commercial ultrasonic transducers designed for air-coupled operation, one of which was the transducer used in the 1999-2000 energy meter design, were also tested for comparison with the special-order devices. The ultrasonic transducers evaluated in the bench tests included:

Commercial Transducers:

1. Siemens DGT speed-of-sound sensor ( $200 \mathrm{kHz}$; 0.5-in. diameter)

2. Massa Products Model E-188-220 (220 kHz; 0.5-in. diameter)

Special-Order Transducers:

3. Ultran Model NCT-505 (500 kHz; 1.0-in. diameter)

4. Ultran Model NCT-510 (1,000 kHz; 0.57-in. diameter)

5. Etalon Model CIA-3525-SB (279 kHz; 1.0 -in. diameter).

Test results, presented in detail in the appendix, showed that the commercial-design transducers were not as effective as the special-order transducers. This was primarily because of their restricted bandwidth (approximately 10\% or less of center frequency). Two of the three special-order transducers provided effective speed-of-sound test results, with the $279 \mathrm{kHz}$ Etalon Model CIA-3525-SB having a bandwidth of $70 \mathrm{kHz}$, giving the best results. In particular, this transducer exhibited very good signal-to-noise ratio over the complete pressure range of 20-100 psia tested. An improved version of this Etalon transducer was received too late for systematic bench testing, but initial tests showed it to be superior to the first Etalon unit because of its wider bandwidth $(128 \mathrm{kHz})$ and equally good signal-to-noise ratio. This transducer, designated Model CIA-3525-SB2, was used in all subsequent tests of the energy meter system. Its performance characteristics are described later in Section 3.2.7. The Ultran Model NTC-510 transducer operating below resonance at a center frequency of $750 \mathrm{kHz}$ gave usable time-of-flight measurements in natural gas at pressures greater than about 50-60 psia, but had sensitivity limitations resulting in a low signal-to-noise ratio in high-methane natural gas at low pressures. The third special-order transducer, Ultran Model NTC-505 operating at $500 \mathrm{kHz}$, had excessive errors in speed of sound because of limited bandwidth, but was capable of providing acceptable reflection signal-to-noise ratio in all of the gas mixtures tested. 
Figures 3.19 and 3.20 show the bench test speed-of-sound performance characteristics of the $279 \mathrm{kHz}$ Etalon Model CIA-3525-SB ultrasonic transducer when operated in a representative natural gas mixture.

The test results shown in Figure 3.19 illustrate the accuracy of speed-of-sound measurements at $279 \mathrm{kHz}$ when operated at room temperature $\left(74^{\circ} \mathrm{F}\right)$ over the pressure range of 20-100 psia. The measured speed of sound was $0.31 \mathrm{ft} / \mathrm{sec}$ higher than the speed of sound calculated using the AGA-8 equation of state [Lomic, Inc. (1997)] for the gas mixture under test. The pressure dependence of the measured values was in accurate agreement with the calculated values. The magnitude of the offset in measured speed of sound and the variations with respect to the experimental mean speed of sound versus pressure approached the practical resolving power of the sensor system based on the digital sampling rate and related time resolution obtained in the correlation analysis. The possible sources of error in these bench tests are: (1) the difference between the actual temperature of the gas in the test chamber and the average gas temperature during each test run on which the calculated speed of sound was based; (2) uncertainty in the exact distance between the two sound reflection faces in the test chamber; and (3) a possible differential phase error between the two reflected signals caused by near-field diffraction effects of the ultrasonic transducer. The ultrasonic pulse waveforms shown in Figure 3.20 illustrate the good signal-to-noise ratio over the pressure range tested, and the frequency spectra indicate that the bandwidth of the received signals is approximately $70 \mathrm{kHz}$ centered at $279 \mathrm{kHz}$. The correlation peak shown in Figure 3.20 had a reasonably well-defined coherence lobe (center positive peak), which is a critical requirement for automatically deriving the difference in time of flight between the two reflected ultrasonic pulses.

The other ultrasonic transducers evaluated in the bench tests exhibited unacceptable performance limitations primarily because of narrow bandwidth (poor time-of-flight resolution) or low sensitivity (poor signal-to-noise ratio). Table 3.3 summarizes the speed-of-sound bench test results for the five ultrasonic transducers.

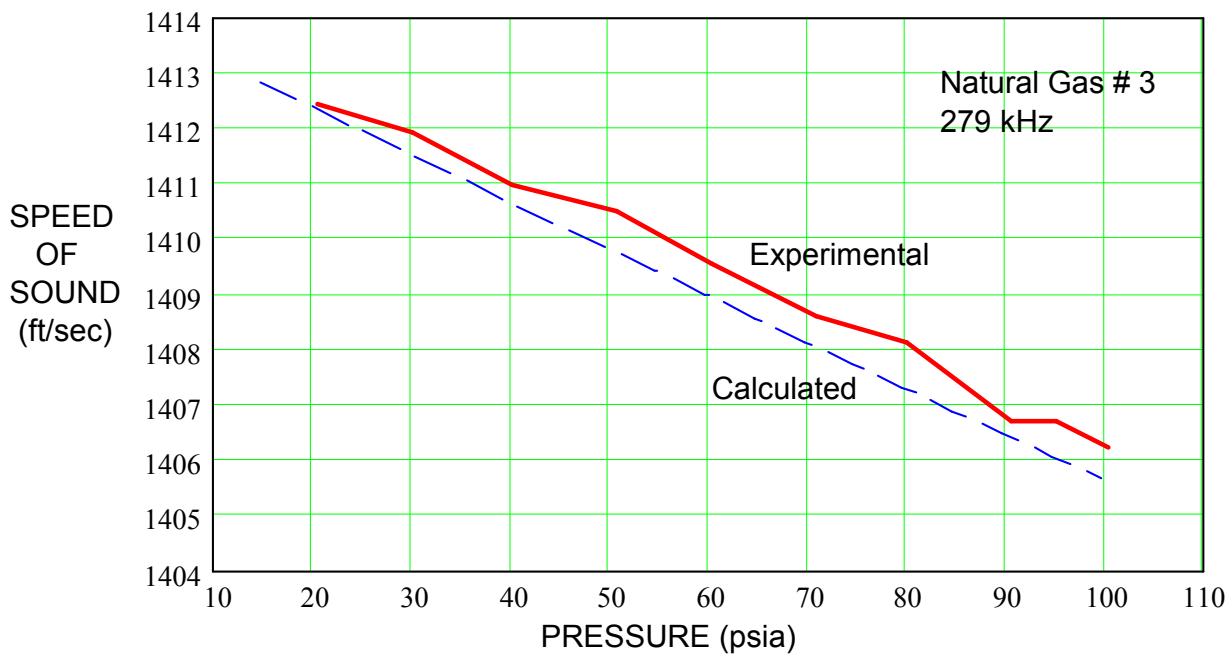

Figure 3.19. Speed of sound vs. pressure: Etalon CIA-3525-SB 279-kHz ultrasonic transducer in Natural Gas \#3. 

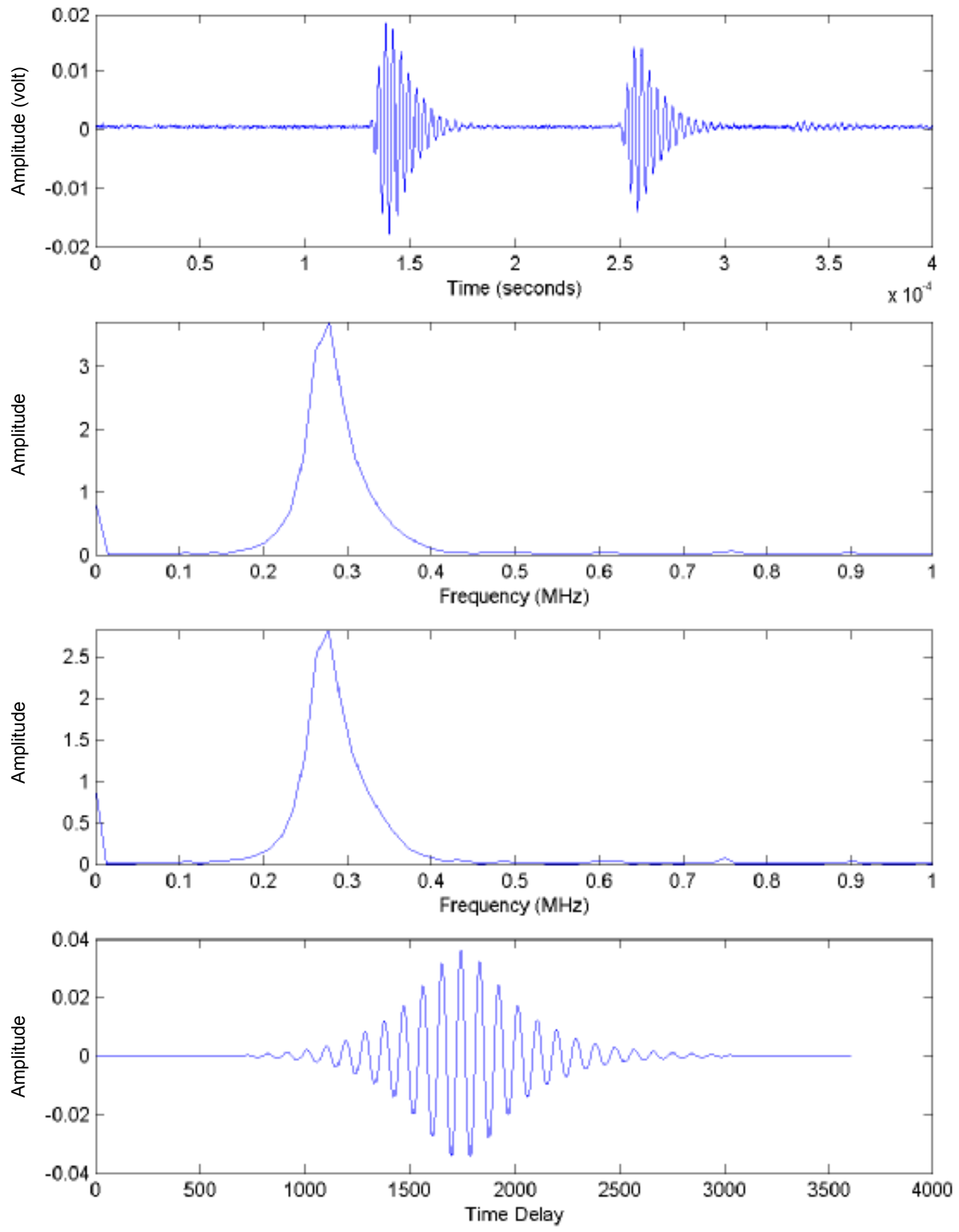

Figure 3.20. Pulse signals, frequency spectra, and cross-correlation function: Etalon CIA-3525-SB 279-kHz ultrasonic transducer in Natural Gas \#3.

Pressure: 60.26 psia; temperature: $73.5^{\circ} \mathrm{F}$. 
Table 3.3. Summary of bench tests on ultrasonic transducers for the speed-of-sound sensor.

\begin{tabular}{|c|c|c|c|c|c|c|c|}
\hline $\begin{array}{l}\text { Transducer } \\
\text { Manufacturer and } \\
\text { Model Number }\end{array}$ & $\begin{array}{l}\text { Peak } \\
\text { Freq. } \\
\text { (kHz) }\end{array}$ & $\begin{array}{l}\text { Bandwidth at } \\
-6 \mathrm{~dB} \text { relative } \\
\text { to Peak Freq. } \\
(\mathrm{kHz})\end{array}$ & $\begin{array}{l}\text { Active } \\
\text { Diam. } \\
\text { (in.) }\end{array}$ & Test Gas & $\begin{array}{l}\text { Speed } \\
\text { Measi } \\
\text { Accur } \\
\%\end{array}$ & $\begin{array}{l}\text { f-sound* } \\
\text { ft/sec }\end{array}$ & Remarks \\
\hline $\begin{array}{l}\text { SIEMENS CORP. } \\
\text { Model DGT }\end{array}$ & 200 & $\begin{array}{l}20 \\
\text { (10\% of peak } \\
\text { frequency) }\end{array}$ & 0.50 & $\begin{array}{l}\text { Nitrogen }(98 \%) \\
\text { Methane }(100 \%) \\
\text { NG }(75 \% \text { Methane })\end{array}$ & $\begin{array}{r}-0.78 \\
-0.96 \\
0.104\end{array}$ & $\begin{array}{l}-9.02 \\
-58.4 \\
1.28\end{array}$ & $\begin{array}{l}\text { Narrow bandwidth caused extended ring-down oscillations and a } \\
\text { broad correlation response that resulted in cycle-skipping when } \\
\text { automatically selecting the time-of-flight correlation peak. This trans- } \\
\text { ducer is not usable in the present speed-of-sound sensor design. }\end{array}$ \\
\hline $\begin{array}{l}\text { MASSA PRODUCTS } \\
\text { Model E-188/220 }\end{array}$ & 220 & $\begin{array}{l}15 \\
(6.8 \% \text { of peak } \\
\text { frequency) }\end{array}$ & 0.50 & $\begin{array}{l}\text { Nitrogen }(98 \%) \\
\text { NG (75\%Methane) }\end{array}$ & $\begin{array}{l}N / A \\
N / A\end{array}$ & $\begin{array}{l}N / A \\
N / A\end{array}$ & $\begin{array}{l}\text { Preliminary tests showed excessive ring-down oscillations caused by } \\
\text { very narrow bandwidth and nonuniform spectral response. Accep- } \\
\text { table test data were obtained only for the NG test gas. This trans- } \\
\text { ducer is not usable in the present speed-of-sound sensor design. }\end{array}$ \\
\hline $\begin{array}{l}\text { ULTRAN LABS, INC. } \\
\text { Model NCT } 505\end{array}$ & 500 & $\begin{array}{l}40 \\
\text { (8\% of peak } \\
\text { frequency) }\end{array}$ & 1.0 & $\begin{array}{l}\text { Nitrogen }(98 \%) \\
\text { NG (75\%Methane) } \\
\text { NG (94\%Methane) }\end{array}$ & $\begin{array}{l}-0.78 \\
-0.20 \\
-0.19\end{array}$ & $\begin{array}{l}-9.02 \\
-2.47 \\
-2.76\end{array}$ & $\begin{array}{l}\text { Higher operating frequency and wider absolute bandwidth than the } \\
\text { lower frequency transducers listed above provided improved time-of- } \\
\text { flight resolution and a somewhat more distinctive correlation peak } \\
\text { without evidence of correlation cycle skipping when automatically } \\
\text { selecting the time of flight. Near-field diffraction effects appear to be } \\
\text { an additional source of speed-of-sound errors. This transducer is not } \\
\text { usable in the present speed-of-sound design. }\end{array}$ \\
\hline $\begin{array}{l}\text { ULTRAN LABS, INC. } \\
\text { Model NCT } 510\end{array}$ & 750 & $\begin{array}{l}350 \\
\text { (50\% of peak } \\
\text { frequency) }\end{array}$ & 0.57 & $\begin{array}{l}\text { Nitrogen }(98 \%) \\
\text { Methane }(100 \%) \\
\text { NG }(75 \% \text { Methane }) \\
\text { NG }(95 \% \text { Methane })\end{array}$ & $\begin{array}{l}\mathrm{N} / \mathrm{A} \\
\mathrm{N} / \mathrm{A} \\
0.005 \\
0.092\end{array}$ & $\begin{array}{l}\mathrm{N} / \mathrm{A} \\
\mathrm{N} / \mathrm{A} \\
0.065 \\
1.30\end{array}$ & $\begin{array}{l}\text { The wide bandwidth of this transducer resulted in an ultrasonic } \\
\text { wavelet having } 3-4 \text { oscillation cycles and a well-defined correlation } \\
\text { peak. The higher operating frequency and distinct correlation peak } \\
\text { provided accurate speed-of-sound measurements. Preliminary tests } \\
\text { in pure nitrogen and pure methane indicated excessive anomalous } \\
\text { absorption losses. High absorption losses were also observed in the } \\
\text { natural gas mixtures. Excessive transducer coupling loss resulted in } \\
\text { poor signal-to-noise ratio in the low range of operating pressures. A } \\
\text { two-way reflection path length of } 1 \text { in. provided the most useful test } \\
\text { results in the natural gas mixtures tested. This transducer is } \\
\text { potentially applicable in the speed-of-sound sensor only at gas } \\
\text { pressures greater than } 100 \text { psia. }\end{array}$ \\
\hline $\begin{array}{l}\text { ETALON, INC. } \\
\text { Model CIA-3525-SB }\end{array}$ & 279 & $\begin{array}{l}70 \\
\text { (25\% of peak } \\
\text { frequency) }\end{array}$ & 1.0 & $\begin{array}{l}\text { Nitrogen }(98 \%) \\
\text { NG (75\%Methane) } \\
\text { NG (95\%Methane) }\end{array}$ & $\begin{array}{l}0.07 \\
-0.06 \\
0.093\end{array}$ & $\begin{array}{l}0.75 \\
-0.74 \\
0.57\end{array}$ & $\begin{array}{l}\text { Medium operating frequency and wider bandwidth than other low } \\
\text { frequency transducers listed above provided good signal-to-noise } \\
\text { ratio, good correlation peak, and accurate speed-of-sound measure- } \\
\text { ments in all gases tested. Test results were obtained with a two-way } \\
\text { reflection path length of } 6.50 \text { inches. This transducer is applicable in } \\
\text { the present speed-of-sound sensor design. }\end{array}$ \\
\hline
\end{tabular}

*The AGA-8 equation of state implemented in the Lomic, Inc. SonicWare ${ }^{\circledR}$ software package was used to calculate the reference speed of sound at the average temperatures and pressures measured in the test chamber. The tabulated values are the experimental offset errors relative to the calculated values. 


\subsubsection{Ultrasonic Transducer and Speed-of-Sound Chamber Design}

Figure 3.21 shows a simplified schematic drawing of the speed-of-sound test chamber developed as part of the 1999-2000 project. This chamber contained a $\frac{1}{2}$-in. diameter ultrasonic transducer (Siemens Corp. Model DGT) and two reflecting targets spaced at 2 in. and 3 in. from the transducer, together with an RTD temperature sensor to measure the gas temperature. Two gas inlets into the chamber were designed to cause the gas to circulate upon entry into the chamber. The chamber was made of aluminum and was capable of withstanding an internal operating pressure greater than $30 \mathrm{psia}$. All speed-of-sound measurements were conducted at 30 psia. The inherent limitations of this chamber and its ultrasonic transducer described earlier imposed performance limitations on the accuracy and reliability of the speed-of-sound measurement. The test chamber was redesigned to eliminate temperature differences that could occur between the test gas and the chamber walls. The chamber was also redesigned to accept the new ultrasonic transducer (Etalon Model CIA-3525-SB2).

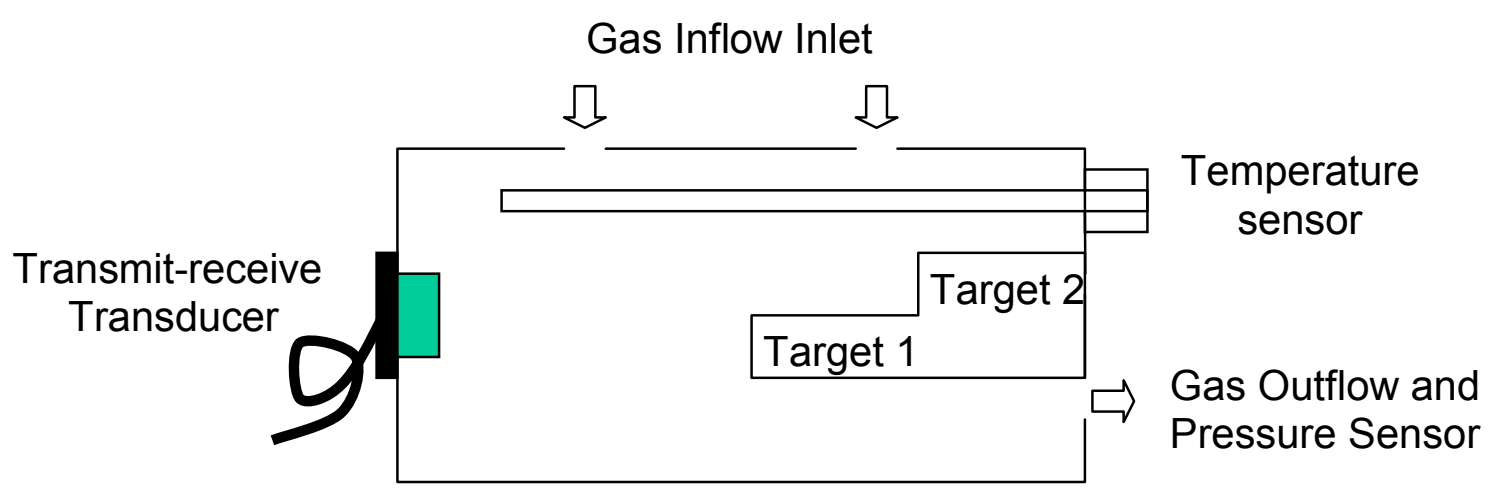

Figure 3.21. Schematic drawing of 1999-2000 speed-of-sound test chamber.

\subsubsection{Ultrasonic Transducer}

The ultrasonic transducer used in the prototype speed-of-sound sensor is a special-order Etalon Model CIA-3525-SB2 air or gas medium transducer having a peak frequency response of $266 \mathrm{kHz}$ and a $-6 \mathrm{~dB}$ bandwidth of $128 \mathrm{kHz}$ (45\% of peak frequency). Figure 3.22 shows a photograph of this transducer with its pressure-sealed coaxial cable mounting insert for use in the speed-of-sound test chamber. The transducer is a piezoelectric composite device capable of reciprocal transmit-receive operation. Efficient air coupling is achieved using a thin elastomeric matching layer on the face of the transducer. The transducer has a 1-in. diameter active face and is mounted in a 1.125-in. diameter by 1.50-in long stainless steel housing with a BNC coaxial connector on the rear. The transducer housing is vented to permit pressure equalization when installed internally within the speed-of-sound test chamber. 


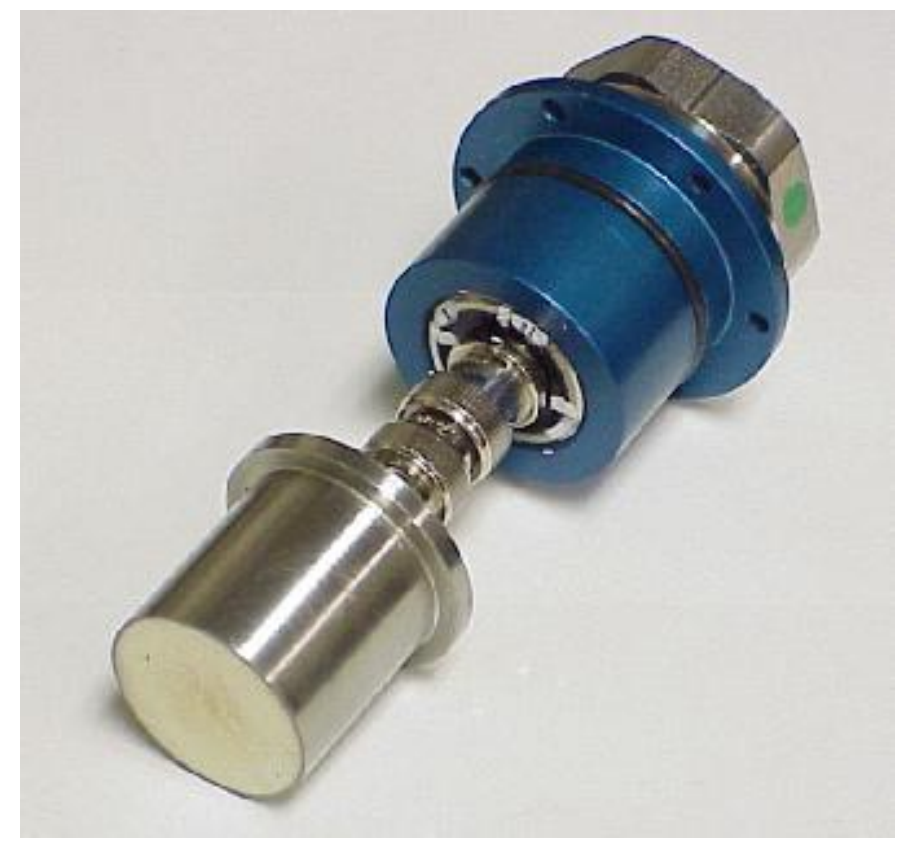

Figure 3.22. Photograph of Etalon Model CIA-3525-SB2 ultrasonic transducer.

\subsubsection{Thermal Effects on 1999-2000 Test Chamber}

The LPL laboratory tests performed on the 1999-2000 speed-of-sound sensor revealed that outdoor ambient temperature changes had a strong effect on the experimentally measured speed of sound in natural gas. That is, even though test gas was continuously flowing through the test chamber, the measured parameters of gas temperature, gas pressure, and ultrasonic pulse travel time did not yield the same speed-of-sound results under quiescent conditions of indoor bench testing and under less controlled outdoor testing conditions on the MRF low pressure flow loop. A main difference in these tests was the nearly isothermal indoor conditions compared with distinctly different temperatures between the gas tapped from the LPL pipeline and the speed-ofsound test chamber within the energy module. Moreover, since the test chamber was designed to minimize any stagnation of gas in the test chamber, a gentle but persistent amount of forced convective heat transfer could occur between the gas and the test chamber body. This heat transfer effect was not observed in the near-isothermal bench test conditions but could readily cause nonuniform temperature conditions in the gas volume within the test chamber whenever the temperature of the gas entering the chamber differed from the temperature of the chamber wall.

Systematic tests were designed and performed to identify the significant factors that could affect the temperature of the gas and, in particular, to determine the cause of any temperature gradient between gas in the center of the chamber and gas near the chamber wall. For this purpose, a factorial test was designed to examine the influence of a number of possible factors that could potentially cause gas temperature measurement discrepancies including: 
- RTD sensor accuracy and response time;

- Heat conduction from the chamber body along the RTD metallic jacket to the resistance sensing element;

- Radiant heat transfer from the chamber wall to the gas;

- Volumetric flow rate of gas through the test chamber;

- Temperature of the test chamber body;

- Temperature of the gas entering the test chamber.

Except for the radiant heat transfer factor, the five remaining factors were considered to have a potential influence on the measured temperature gradients in the gas under outdoor ambient conditions.

Four controlled bench tests were designed to determine the significance of the five factors under consideration. The test chamber was modified to accept two four-wire RTD sensor probes mounted in either aluminum (thermally conductive) bushings or nylon (thermally insulating) bushings with one probe located on the chamber axis and one probe located near the cylindrical chamber wall. Two different pairs of RTD probes were used in each of the probe mounting configurations. The tests were performed using nitrogen as the test gas with a heat exchanger tubing coil and electric heating element located between the gas supply and the test chamber to adjust the inlet gas temperature. A pressure regulator and a rotameter flow sensor were used to adjust and measure the volumetric flow through the chamber. The test chamber body was wrapped with an electric heating tape to adjust the temperature of the chamber. Thermocouple sensors were used to measure and monitor the inlet gas temperature, the outlet gas temperature, and the chamber body temperature. Figure 3.23 shows a photograph of the experimental test setup.

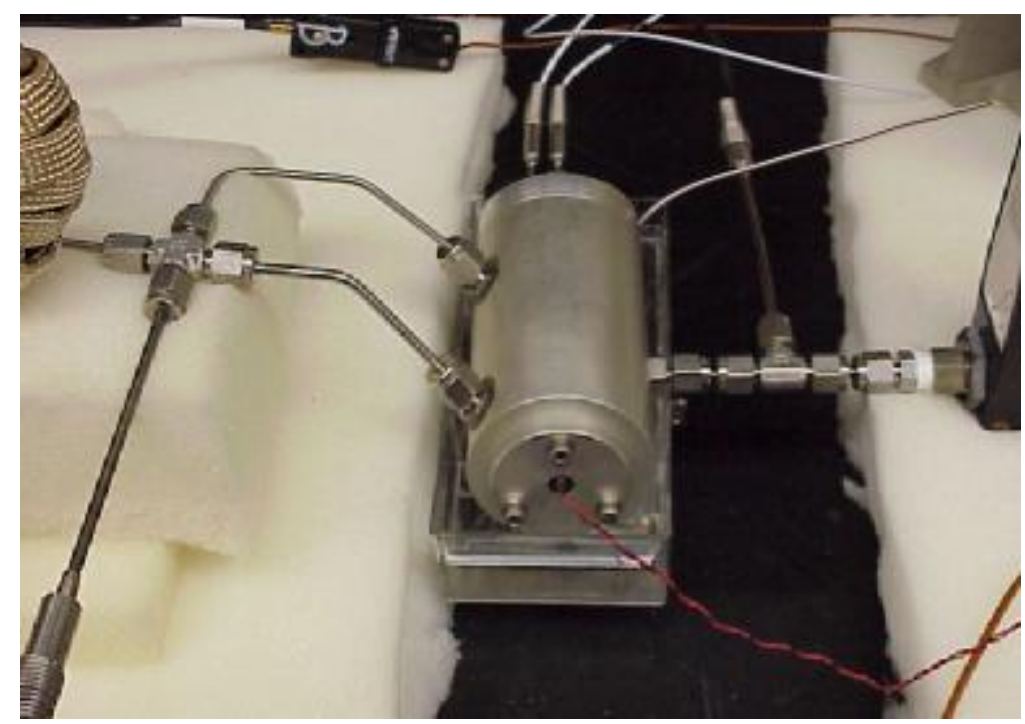

Figure 3.23. Photograph of thermal effects test setup. 
A sufficient number of high and low combinations of inlet gas temperature and test chamber body temperature were specified for use in each test run to allow the influence of changes in the variable factors on the temperature difference between the two RTD sensors to be determined. These data were analyzed using conventional statistical analysis tools applied to the RTD temperature difference data, including analysis of variance, to isolate the effects of each factor.

Evaluation of the test results revealed that only the test chamber temperature and the gas flow rate had statistically significant effects on the temperature gradient in the gas. These factors were dominant parameters in the tests since the chamber body serves as either a heat source or heat sink to the gas and the flow rate governs the motions of the gas in the chamber and, hence, promotes heat transfer between the gas and the chamber. The remaining three factors (RTD sensor accuracy, RTD mounting, and inlet gas temperature) did not have a statistical influence on the temperature gradient in the gas.

Based on the results of these tests, the 1999-2000 test chamber was concluded to be fundamentally susceptible to errors associated with changes in ambient temperature of the energy meter module and temperature differences between the energy meter module and the gas entering the module. To overcome this problem, a new design of the speed-of-sound test chamber was devised whereby the gas is equilibrated to match the ambient temperature of the test chamber body before it enters the test chamber for ultrasonic measurements.

\subsubsection{Speed-of-Sound Test Chamber}

A specialized speed-of-sound test chamber was designed for the 2000-2001 energy meter module. This chamber emphasizes the capability to precondition the gas temperature and gas throughput flow to provide stable and uniform conditions for accurate speed-of-sound measurements. Figure 3.24 shows the design layout of the test chamber. The ultrasonic transducer is mounted entirely within the chamber with small openings provided around the transducer housing to allow gas pressure to reach the back of the transducer for the purpose of equalizing the pressure on both sides of the active piezoelectric element. The chamber is designed to operate at pressures in the range of 50-100 psia provided by a pressure regulator attached to a tap on the gas pipeline source. A machined dual-step reflector pedestal is mounted in the opposite end from the transducer to provide two half-circle reflector faces having a 0.500in. fixed separation distance. This pedestal is made of Super Invar ${ }^{\circledR}$ material to minimize the effects of temperature on the reflector spacing. The distance between the transducer face and the nearest reflector face is designed to be 2.0 in. but may be made smaller by adding different cylindrical spacers attached to the reflector pedestal base. A gas outlet port and a sealed port for inserting an RTD temperature probe into the central zone of the test chamber are located at the reflector end of the chamber.

Before entering the test volume of the chamber, the gas travels through a covered helical groove machined into the outer surface of the aluminum chamber body to allow the gas to equilibrate to the temperature of the metallic chamber. The chamber acts as a semi-stable heat sink (subject to diurnal changes in ambient temperature). On exiting the helical groove, the gas enters an annular space formed by the inner chamber wall and a 0.1 -in. thick porous sleeve made of $40 \%$ density Duocel ${ }^{\circledR}$ aluminum foam. This porous sleeve serves as a diffusion interface to prevent the formation of any persistent gas circulation patterns in the chamber. The overall length of the speed-of-sound test chamber is $7.75 \mathrm{in}$. and the outside diameter is 2.75 inches. The 
helical groove is covered and pressure sealed by a close-tolerance 0.125 -in. thick cylindrical sleeve made of Black Almagon ${ }^{\mathbb{B}}$ graphite-epoxy material. The inner diameter of the chamber is $2.25 \mathrm{in}$. and the combined wall thickness is $0.25 \mathrm{inch}$. The helical groove is $0.094 \mathrm{in}$. wide by $0.062 \mathrm{in}$. deep and provides an effective gas flow path length of approximately $345 \mathrm{in}$. which, for gas throughput volume flow at $0.20 \mathrm{l} / \mathrm{min}$. (laminar flow in the helical groove), gives a thermal equilibration dwell time of approximately 10 seconds. As a result of this gas temperature preconditioning design, the temperature of the gas entering the test chamber is, for practical purposes, the same temperature as the body of the chamber. The RTD temperature probe measures the gas temperature in the sound propagation zone of the chamber volume. Figure 3.25 is a cutaway drawing of the speed-of-sound chamber showing the details of the design described above.

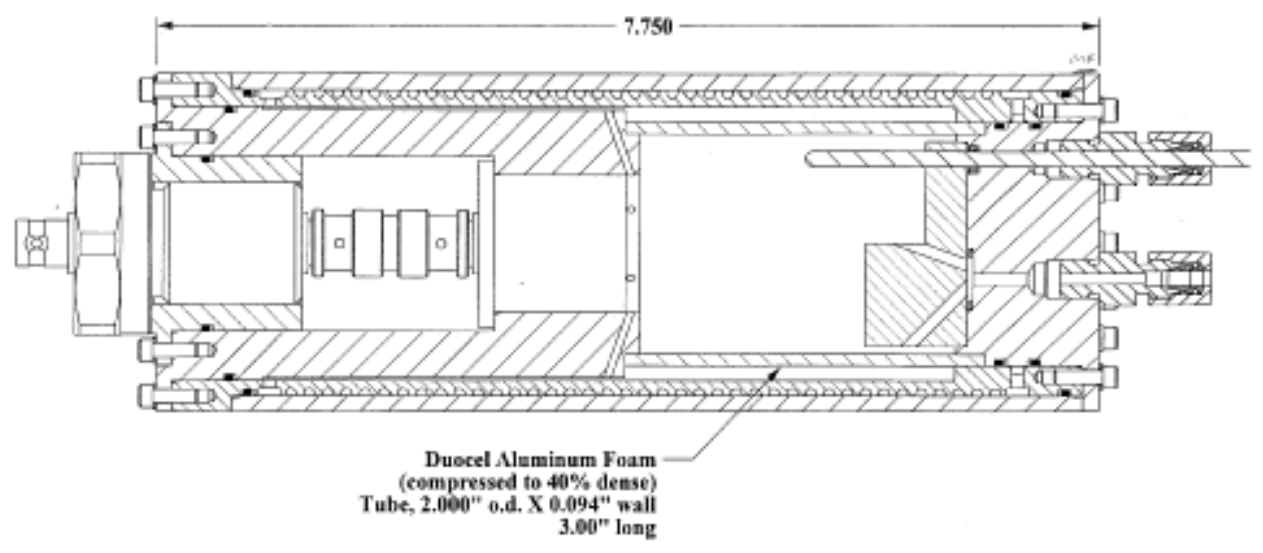

Figure 3.24. Layout drawing of speed-of-sound test chamber.

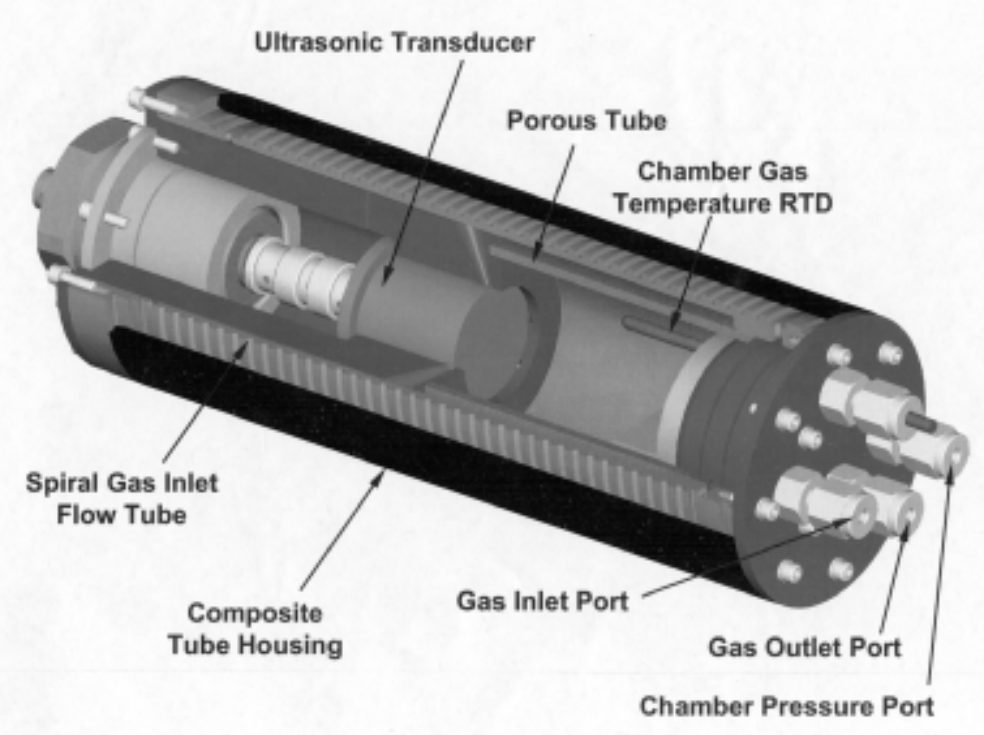

Figure 3.25. Cutaway drawing of speed-of-sound test chamber. 


\subsubsection{Speed-of-Sound Error Analysis}

Assuming uniform temperature conditions and negligible motions in the gas contained in the test chamber, the measured speed of sound is subject to two sources of error: (1) uncertainties in the distance measurement, $(\Delta \mathrm{D})$, and (2) uncertainties in the time measurement, $(\Delta \mathrm{t})$.

\subsubsection{Uncertainties in Distance $(\Delta D)$}

The distance between the two reflector targets was measured using a micrometer having an accuracy of \pm 0.0001 inch. This inaccuracy in the distance measurement can produce a maximum error of $\pm 1.6 \mathrm{in} / \mathrm{sec}$ in the speed of sound, an error well within the accuracy required for the energy meter speed-of-sound measurements.

Thermal expansion of the target material can also affect the distance between the targets. To reduce this effect, the targets were made from a single piece of Super Invar ${ }^{\mathbb{B}}$, which has a very low coefficient of thermal expansion $\left(3.389 \times 10^{-6} /{ }^{\circ} \mathrm{F}\right)$. For the 0.500 -in. target separation distance, the distance will vary by $1.69 \times 10^{-6}$ in. per ${ }^{\circ} \mathrm{F}$. For operation over a temperature extreme range of 0 to $120^{\circ} \mathrm{F}$, the distance between the targets will change by $2.1 \times 10^{-4} \mathrm{inch}$. Such a change in the distance between the targets can produce an error of $7.2 \mathrm{in} . / \mathrm{sec}$. in the measured speed of sound. Although this error is not negligible, it is predictable by using the measured temperature of the gas and may be compensated for as part of the ultrasonic transit time analysis.

\subsubsection{Uncertainties in Speed-of-Sound Measurement}

The accuracy of the speed-of-sound sensor is dependent on measurements of gas temperature and pressure, and the timing of the received ultrasonic reflection waveform. By design, the gas entering the speed-of-sound test chamber is equilibrated to the temperature of the test chamber prior to the ultrasonic measurement. The sensor is calibrated by adjusting the effective spacing between the two ultrasonic reflection targets to match the theoretical speed of sound in a gas of known composition (calculated using the AGA-8 equation of state via SonicWare $^{\circledR}$. This calibrated value of $\Delta \mathrm{D}$ is then used in all subsequent data analysis. However, the actual spacing is sensitive to temperature through the thermal expansion coefficient of the material comprising the target block. Thus, the speed-of-sound measurement is affected by the combined temperature effects on the gas and on the target material. By selecting Super Invar ${ }^{\circledR}$ as the target material, the latter effect is minimized. The temperature and pressure effects on the gas may be estimated to within sufficient accuracy by assuming the error effects to be dominated by the methane content of the gas and representing the gas by the virial equation of state through the temperature dependence of the second virial coefficient.

Expressed in terms of the virial equation, the speed of sound in methane is

$$
\frac{v_{g}{ }^{2}}{v_{o}{ }^{2}}=1+2 p\left[B+\frac{R}{C_{v}^{o}} \frac{\partial(B T)}{\partial T}+\frac{R^{2} T}{C_{v}^{o} C_{p}^{o}} \frac{\partial^{2}(B T)}{\partial T^{2}}\right]
$$


where

$$
\begin{aligned}
B= & B=a_{1}+a_{2}\left(\frac{T_{o}}{T}-1\right)+a_{3}\left(\frac{T_{o}}{T}-1\right)^{2}+a_{4}\left(\frac{T_{o}}{T}-1\right)^{3} \\
R= & \text { universal gas constant } \\
C_{\mathrm{v}}{ }^{\mathrm{o}}= & \begin{array}{l}
\text { specific heat capacity of methane at constant volume at the reference } \\
\text { condition }
\end{array} \\
C_{\mathrm{p}}{ }^{\mathrm{o}}= & \begin{array}{l}
\text { specific heat capacity of methane at constant pressure at the reference } \\
\text { condition }
\end{array} \\
\mathrm{v}_{0}= & v_{o}=\sqrt{\frac{\gamma R T}{M}}=\text { speed of sound in the gas assuming ideal gas conditions } \\
\mathrm{M}= & \text { molar mass of the gas } \\
\mathrm{a}_{1}, \mathrm{a}_{2}, \mathrm{a}_{3}, \mathrm{a}_{4}= & -43,-114,-19,-7 \mathrm{~cm}^{3} / \text { mole, respectively, for pure methane at a } \\
& \text { reference temperature of } T_{o}=298.15 \mathrm{~K}
\end{aligned}
$$

The terms in brackets in Equation (3.18) are only a function of temperature, which, upon expansion, become:

$$
F(T)=\left\{\begin{array}{l}
\left(a_{1}-a_{2}-a_{3}+a_{4}\right)+\left(a_{2}-2 a_{3}+3 a_{4}\right)\left(\frac{T_{o}}{T}\right)+\left(a_{3}-3 a_{4}\right)\left(\frac{T_{o}}{T}\right)^{2}+a_{4}\left(\frac{T_{o}}{T}\right)^{3} \\
+\frac{R}{C_{v}^{o}}\left[\left(a_{1}-a_{2}-a_{3}+a_{4}\right)-\left(a_{3}-3 a_{4}\right)\left(\frac{T_{o}}{T}\right)^{2}-a_{4}\left(\frac{T_{o}}{T}\right)^{3}\right] \\
+\frac{R^{2}}{C_{v}^{o} C_{p}^{o}}\left[\left(a_{3}-3 a_{4}\right)\left(\frac{T_{o}}{T}\right)^{2}-\frac{3}{2} a_{4}\left(\frac{T_{o}}{T}\right)^{3}\right]
\end{array}\right\}
$$

Therefore, the fractional error sensitivity of speed of sound in terms of errors in pressure and temperature in Equation (3.18) is

$$
\frac{d v_{g}}{v_{g}}=\frac{F(T)}{1+2 p F(T)} d p+\frac{\left.p \frac{\partial F(T)}{\partial T}\right|_{p}}{1+2 p F(T)} d T
$$


and the combined worst-case error associated with measurement errors $\Delta \mathrm{p}$ and $\Delta \mathrm{T}$ is

$$
\Delta v_{g T P}=v_{g} \sqrt{\frac{F(T)}{1+2 p F(T)} \Delta p+\frac{\left.p \frac{\partial F(T)}{\partial T}\right|_{p}}{1+2 p F(T)} \Delta T}
$$

The error in speed of sound caused by thermal expansion of the Super Invar ${ }^{\circledR}$ reflector target block is

$$
\Delta v_{E X}=\frac{\alpha_{i n v} \Delta T}{1+\alpha_{i n v}\left(T-T_{o}\right)} v_{g}
$$

where $\alpha_{i n v}=3.389 \times 10^{-6} /{ }^{\circ} \mathrm{F}=$ coefficient of thermal expansion of Super Invar ${ }^{\circledR}$ material.

The digital sampling rate should be sufficiently high that the combined errors caused by gas temperature and pressure, thermal expansion, and digital sampling on the speed of sound do not exceed the overall error tolerance in speed of sound predicted by the energy meter analysis algorithm. Therefore, for the algorithm error tolerance of $\Delta \mathrm{v}_{\mathrm{ALG}}=0.8 \mathrm{ft} / \mathrm{sec}$, the digital sampling error expressed in terms of the speed-of-sound error must be less than

$$
\begin{aligned}
\Delta v_{S A M} & =\sqrt{\Delta v_{A L G}{ }^{2}-\left(\Delta v_{g T P}{ }^{2}+\Delta v_{E X}{ }^{2}\right)} \\
& =0.511 \mathrm{ft} / \mathrm{sec}
\end{aligned}
$$

and the associated time sampling interval is

$$
\begin{aligned}
\Delta t_{S A M} & =\frac{\Delta v_{S A M}}{v_{g}}\left(\frac{2 \Delta D}{v_{g}}\right) \\
& =2 \times 10^{-8} \mathrm{sec} .
\end{aligned}
$$

Therefore, the minimum time sampling frequency is

$$
\begin{aligned}
f_{S A M} & =\frac{1}{\Delta t_{S A M}} \\
& =50 \mathrm{MHz}
\end{aligned}
$$




\subsubsection{Sensor Calibration}

The speed of sound in the test gas is determined by the ratio of the distance spacing between the two reflector faces, $\Delta \mathrm{D}$, and the measured time difference, $\Delta \tau$, between the two pulse reflections. That is, the speed of sound is derived from

$$
v_{g}=\frac{2 \Delta D}{\Delta \tau}
$$

This simple relationship involving only one fixed dimensional parameter of the system $(\Delta \mathrm{D})$ provides a convenient numerical factor by which the speed-of-sound measurements may be calibrated to match the known speed of sound of a reference gas tested in the system. The Etalon Model CIA-3525-SB $(279 \mathrm{kHz})$ transducer provided very accurate results when using the actual reflector pedestal spacing, $\Delta \mathrm{D}$, in combination with the measured pulse reflection time difference, $\Delta t$. Only a very small numerical adjustment in $\Delta \mathrm{D}$ was required to match the measured speed of sound with that calculated for the test gas.

This method of calibration was applied to the speed-of-sound sensor in the prototype energy meter using a certified natural gas mixture (Natural Gas \#3 listed in the appendix) as the calibration reference gas. For this purpose, the reference gas was fed to the energy meter module via a manually adjusted regulator to purge the chamber of any previous gas content, and then set to provide a flow rate of $0.20 \mathrm{l} / \mathrm{min}$ at a chamber pressure of approximately $100 \mathrm{psia}$. Speed-ofsound measurements together with simultaneous gas pressure and temperature readings were recorded at one-minute intervals for 15 minutes. The experimental speed of sound for each measurement was derived using the relationship given by Equation (3.25) with the physically measured value of $\Delta D$ and the time difference, $\Delta \tau$, derived from the ultrasonic pulse time-offlight measurements. The speed of sound in the reference gas was calculated for the recorded pressure and temperature conditions in the sensor. The calculated speed of sound was subtracted from the experimental value and the difference subjected to a trial-and-error optimization process expressed by

$$
\text { “Adjust } \Delta D \text { until }\left[\left(v_{\mathrm{g}}\right)_{\text {meas }}-\left(v_{\mathrm{g}}\right)_{\text {calc }}\right]^{2} \Rightarrow \text { a minimum value" }
$$

which returns the calibration quantity, $\Delta \mathrm{D}_{\mathrm{CAL}}$, in a manner corresponding to a generalized least squares fitting procedure without approximating the data using a regression trend line. The resulting value of $\Delta \mathrm{D}_{\mathrm{CAL}}$ was then used to determine the speed of sound, $\left(\mathrm{v}_{\mathrm{g}}\right)_{\mathrm{CAL}}$, in all subsequent flow loop tests. Similar but secondary standard calibration checks were also performed using recorded gas chromatography analysis data on the test gases to derive $\left(\mathrm{vg}_{\mathrm{g}}\right)_{\text {calc }}$ to compare the speed-of-sound measurements using the reference-gas-calibrated energy meter module with measurements using conventional (GC-composition) calibrations.

Figures 3.26 and 3.27 show the results obtained using the calibration procedure described above during the MRF HPL tests performed on August 24, 2001. The pre-test and post-test calibrations were performed at temperatures of $77.2^{\circ} \mathrm{F}$ and $113.2^{\circ} \mathrm{F}$ and exhibit standard 
deviations of $0.121 \mathrm{ft} / \mathrm{s}$ and $0.0503 \mathrm{ft} / \mathrm{s}$, respectively. The corresponding best-fit values of $\Delta \mathrm{D}_{\mathrm{CAL}}$ were 0.99977 in. and 0.99965 in., corresponding to a difference in the reflection path of $122 \mu \mathrm{in}$., and the effective thermal sensitivity of $\Delta \mathrm{D}_{\mathrm{CAL}}$ is $3.389 \times 10^{-6} /{ }^{\circ} \mathrm{F}$. The error in the derived speed of sound is proportional to the dimensional sensitivity; for example, the $+36^{\circ} \mathrm{F}$ change in temperature from the pre-test to the post-test calibration conditions imposes a speed-of-sound error of $3.389 \times 10^{-6} /{ }^{\circ} \mathrm{F}\left(+36^{\circ} \mathrm{F}\right)(1411 \mathrm{ft} / \mathrm{s})=+0.172 \mathrm{ft} / \mathrm{s}$. Figure 3.27 also illustrates, in a qualitative way, the ability of the speed-of-sound sensor to track temporal changes in the energy module caused when the module was shaded from the sun for a period of about 3 minutes.

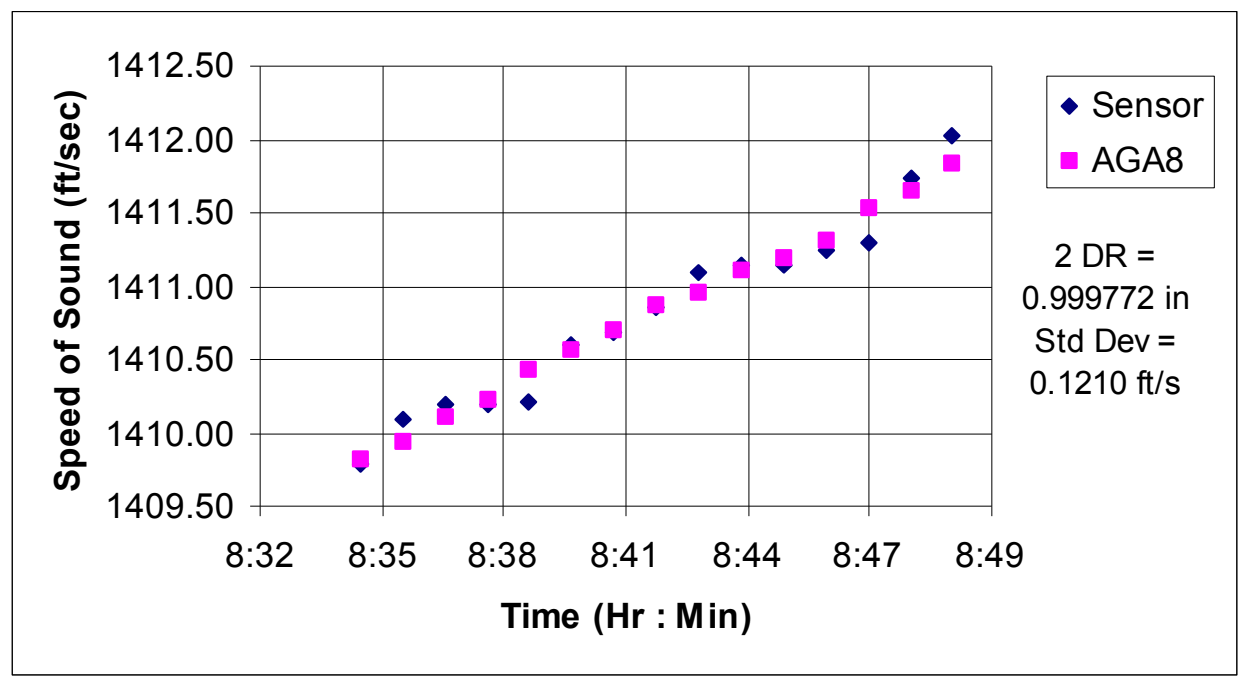

Figure 3.26. Pre-test calibration of speed-of-sound sensor on MRF HPL.

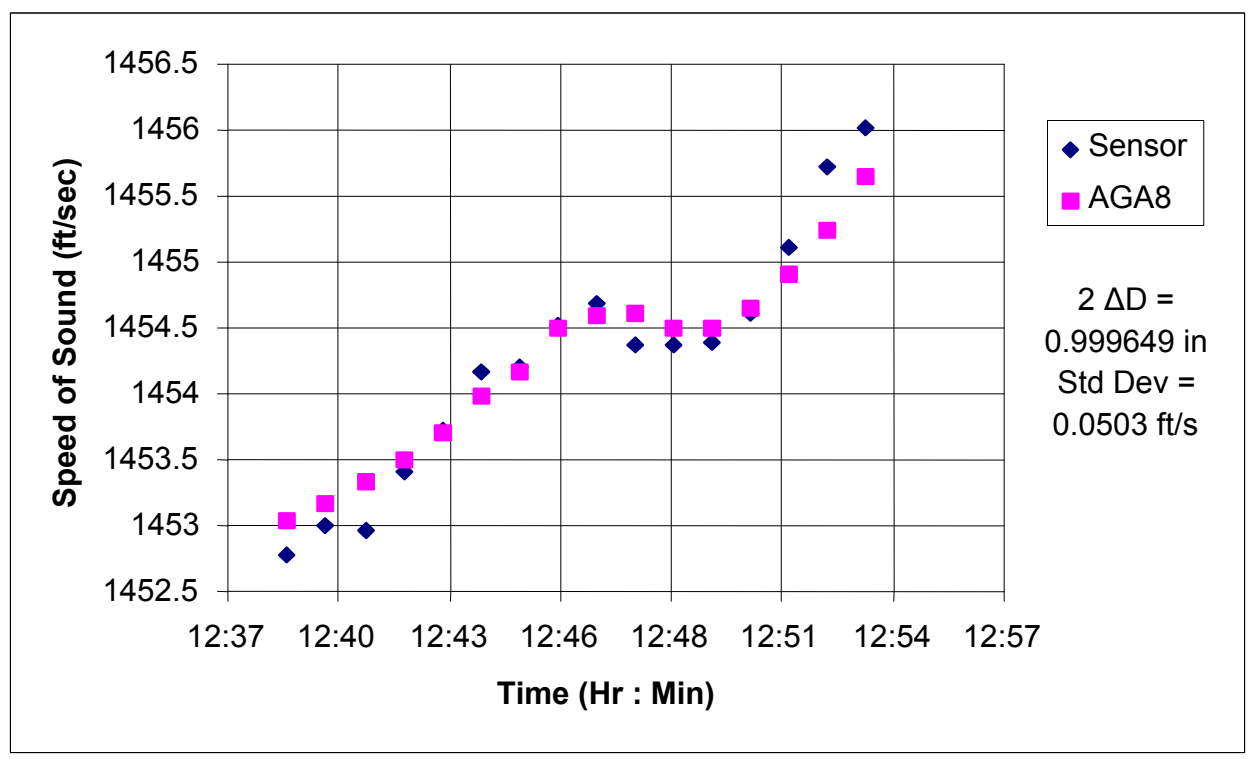

Figure 3.27. Post-test calibration of speed-of-sound sensor on MRF HPL. 


\subsubsection{Speed-of-Sound Measurements in Natural Gas}

Controlled flow-loop tests were conducted to evaluate the prototype energy meter module containing the speed-of-sound sensor operating with samples of commercial natural gas and similar gas samples containing added amounts of $\mathrm{CO}_{2}$. In these tests, gas from the flow loop was tapped via a manually adjusted pressure regulator valve for use in the speed-of-sound sensor of the energy meter. Gas chromatograph data were recorded periodically during the various flow loop tests to provide reference information on the gas composition.

Data related to the speed-of-sound sensor measurements during each flow loop test run included:

1. Test run time (date, hour, minute, second)

2. Gas temperature in speed-of-sound test chamber $\left({ }^{\circ} \mathrm{F}\right)$

3. Gas pressure in speed-of-sound test chamber (psia)

4. Ultrasonic pulse reflection waveforms (via a Tektronix TDS-3012 digital oscilloscope)

5. Experimental speed of sound computed by the energy meter module data analysis system

6. MRF gas chromatograph readings of flow loop gas concentrations

\subsubsection{High-Pressure Loop Tests}

Tests performed in the MRF HPL used normal natural gas, with a fixed composition as shown in Table 3.4 at different temperature, pressure, and flow conditions. Ambient temperatures in the energy meter module during the various test runs ranged from about $78^{\circ} \mathrm{F}$ during early morning tests to about $125^{\circ} \mathrm{F}$ during mid-afternoon tests over the four-day testing period. Natural gas was supplied to the energy meter module via a regulator valve adjusted to provide a throughput flow rate of $0.20 \mathrm{l} / \mathrm{min}$ at a chamber pressure of approximately 100 psia. Data were recorded during 15-minute test runs after each HPL test condition was stabilized. In each test, readings of gas temperature, pressure, computed speed of sound, and digitized ultrasonic pulse reflection waveforms were recorded at one-minute time intervals. These test runs provided a minimum of 15 independent measurements from which experimental values of speed of sound could be determined.

Figure 3.28 shows an example of the ultrasonic pulse waveforms, frequency spectra, and correlation response indicating the time-of-flight difference between the two reflected pulses. These data were recorded during the second day of the four-day testing sequence and are representative of the ultrasonic signals observed during all of the HPL test runs.

The ultrasonic transducer used in the energy meter throughout the flow loop tests was the Etalon Model CIA-3525-SB2 having a peak frequency response of $266 \mathrm{kHz}$ and a $-6 \mathrm{~dB}$ (fullwidth-half-height) bandwidth of $128 \mathrm{kHz}$. The waveforms in Figure 3.28 indicate that the reflected pulses consisted of approximately three oscillations, followed by a weak $(20 \%$ amplitude) reverberation sequence that occurred within the transducer structure as part of the transmitted source signal. The second reflection pulse amplitude was approximately $83 \%$ of the first reflection corresponding to the combined difference in geometrical spreading and absorption loss that occurred over the 0.500 -in. differential propagation path length between the two reflector faces. Both reflections had a peak-signal-to-rms-noise ratio greater than $40 \mathrm{~dB}$. The frequency spectra of the two reflections were essentially identical, with indicated $-6 \mathrm{~dB}$ 
bandwidths of $118.5 \mathrm{kHz}$. The coherence lobe of the correlation response was approximately $42 \%$ greater in amplitude than the adjacent side lobes to provide a reliable indication of the differential time of flight between the two pulses. The weak reverberant features in the source pulse waveform produced only secondary correlation effects with an amplitude of about $20 \%$ of the primary coherence lobe. Essentially all of the ultrasonic pulses recorded in the HPL tests exhibited these signal characteristics.

Table 3.4. Gas composition used in HPL laboratory tests of energy module.

\begin{tabular}{|l|c|}
\hline Gas Constituent & $\begin{array}{c}\text { Composition } \\
\text { (mol\%) }\end{array}$ \\
\hline Methane & 95.5849 \\
\hline Ethane & 1.57689 \\
\hline Carbon Dioxide & 1.35659 \\
\hline Nitrogen & 1.17472 \\
\hline Propane & 0.20207 \\
\hline Isobutane & 0.025325 \\
\hline n-Butane & 0.043202 \\
\hline Isopentane & 0.013219 \\
\hline n-Pentane & 0.012358 \\
\hline n-Hexane & 0.010425 \\
\hline n-Heptane & 0.009935 \\
\hline n-Octane & 0.005818 \\
\hline n-Nonane & 0.001635 \\
\hline
\end{tabular}



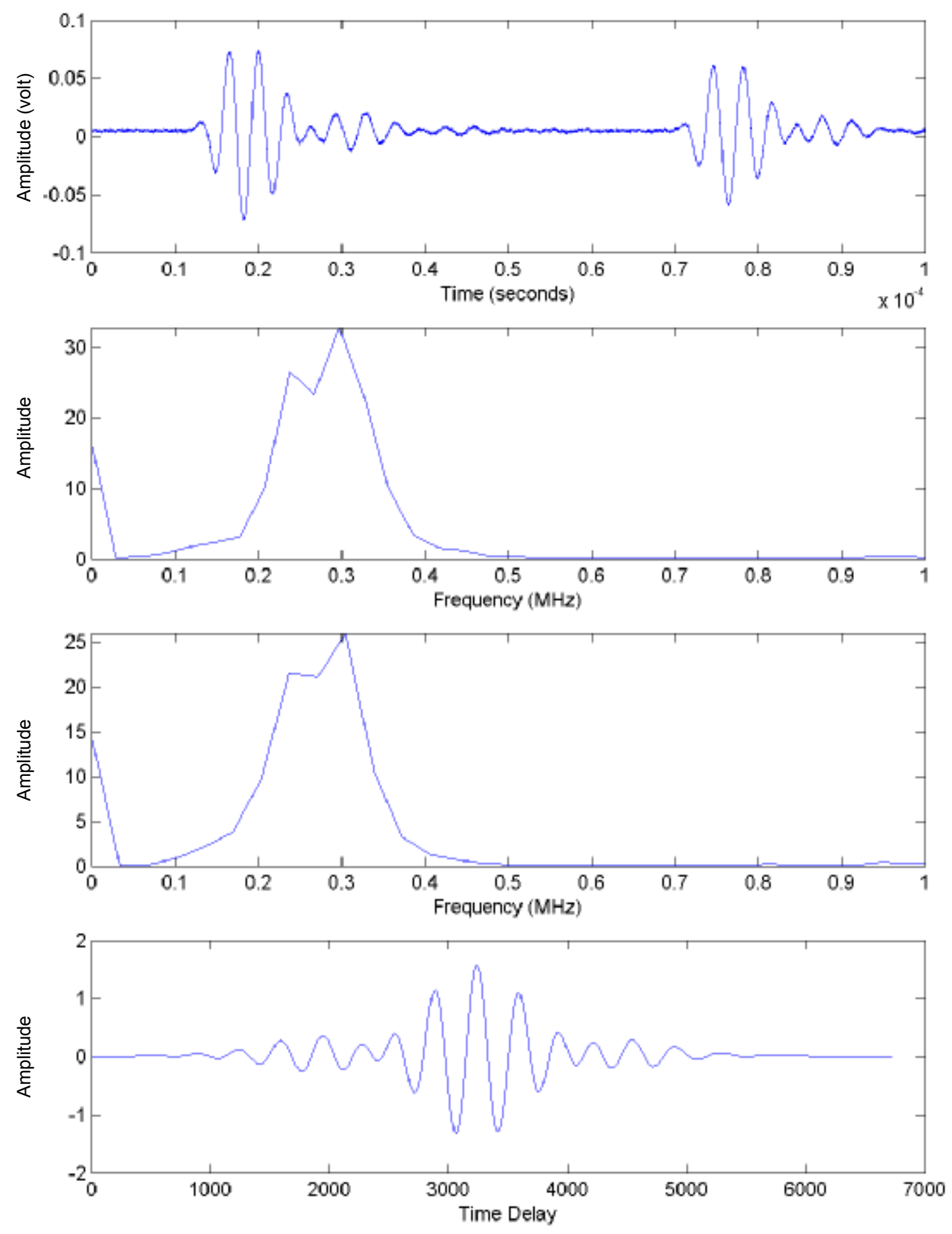

Figure 3.28. Pulse signals, frequency spectra, and cross-correlation function: Etalon CIA-3525-SB2 266-kHz ultrasonic transducer in MRF HPL gas.

Pressure: 99.02 psia; temperature: $82.26^{\circ} \mathrm{F}$. 
Figures 3.29 through 3.36 present test results from eight HPL runs, selected from a total of 24 runs, to illustrate the accuracy and response time of the speed-of-sound sensor. All of these data showed a positive offset between the experimental measurements and the calculated values of speed of sound ranging from about $0.8 \mathrm{ft} / \mathrm{s}$ to about $1.5 \mathrm{ft} / \mathrm{s}$. Part of this error was accounted for by the thermal sensitivity of the sensor $\left(3.389 \times 10^{-6} /{ }^{\circ} \mathrm{F}\right)$, and the remainder was attributed to velocity dispersion in the gas mixture and other possible sources of error in the energy meter module such as ultrasonic near-field differential phase shift and calibration errors in the temperature and pressure sensors. Digital resolution in the data logging and ultrasonic waveform recording processes was the primary cause of the scatter in the measured speed of sound, whereas the other error effects mentioned above were cumulative in a positive sense to potentially account for the observed offset. Except for the test runs shown in Figures 3.33 and Figure 3.35, the speed of sound increased monotonically with time. This trend accurately tracked the rise in ambient atmospheric temperature and, hence, the temperature in the energy meter module. The variation with time shown in Figure 3.36 was caused when the energy meter enclosure was temporarily opened, resulting in a small decrease in ambient temperature of the speed-of-sound test chamber over a period of 8-9 minutes. In all cases, the derived speed-ofsound sensor was able to accurately track the time rate of change without lag.

The simplified model of velocity dispersion in methane-dominant gas mixtures discussed in Section 3.2.2.3 implied that at an ultrasonic signal frequency of $250 \mathrm{kHz}$ and a 5-in. reflection path length, the dispersion error was in the range of about $0.5-0.6 \mathrm{ft} / \mathrm{s}$ at pressures of 90-100 psia. The differential phase shift between the two reflected pulses caused by near-field diffraction as discussed in Section 3.2.2.2 was approximately $0.45 \mathrm{deg}$ at $266 \mathrm{kHz}$ and was equivalent to an error of $0.112 \mathrm{ft} / \mathrm{s}$ in speed of sound. The temperature sensitivity error in speed of sound for the calibration reference temperature of $77.2^{\circ} \mathrm{F}$ and a $36^{\circ} \mathrm{F}$ rise was approximately $0.172 \mathrm{ft} / \mathrm{s}$. Thus, the estimated total cumulative error relevant to the data shown in Figures 3.33 through 3.40 was $0.172+0.112+0.600=0.88 \mathrm{ft} / \mathrm{s}$, which was sufficient to account for most of the offset errors observed in the HPL tests. The dispersion error was the largest contribution to the total error and, because of the simplicity of the model on which it was based, that contribution had the greatest uncertainty and should be investigated further in order to accurately account for the offset bias in the speed-of-sound measurements.

\subsubsection{Low-Pressure Loop Tests}

Tests performed in the MRF LPL involved several natural gas compositions, including normal commercial gas and commercial gas with added $\mathrm{CO}_{2}$ content at various temperatures, pressure, and flow rates. The temperature of the energy meter module during the various test runs ranged from about $81^{\circ} \mathrm{F}$ to about $97^{\circ} \mathrm{F}$ during the four-day testing period. Natural gas was supplied to the energy meter module through a regulator adjusted to provide a flow rate of $0.20 \mathrm{l} / \mathrm{min}$ at chamber pressures of approximately 50 psia and 100 psia. Data were recorded during 15-minute test runs after each test condition was stabilized. In each test, readings of gas temperature, pressure, and digitized ultrasonic pulse reflection waveforms were recorded at oneminute time intervals. These tests provided a minimum of 15 independent measurements from which experimental values of speed of sound could be determined. The addition of $\mathrm{CO}_{2}$ resulted in gas compositions having a speed of sound that varied from $1360 \mathrm{ft} / \mathrm{sec}$ to $1445 \mathrm{ft} / \mathrm{sec}$. The gas composition measured by the MRF GC provided information on the loop gas composition throughout all of the tests. Table 3.5 lists one typical gas composition used in the LPL tests prior to adding $\mathrm{CO}_{2}$. 


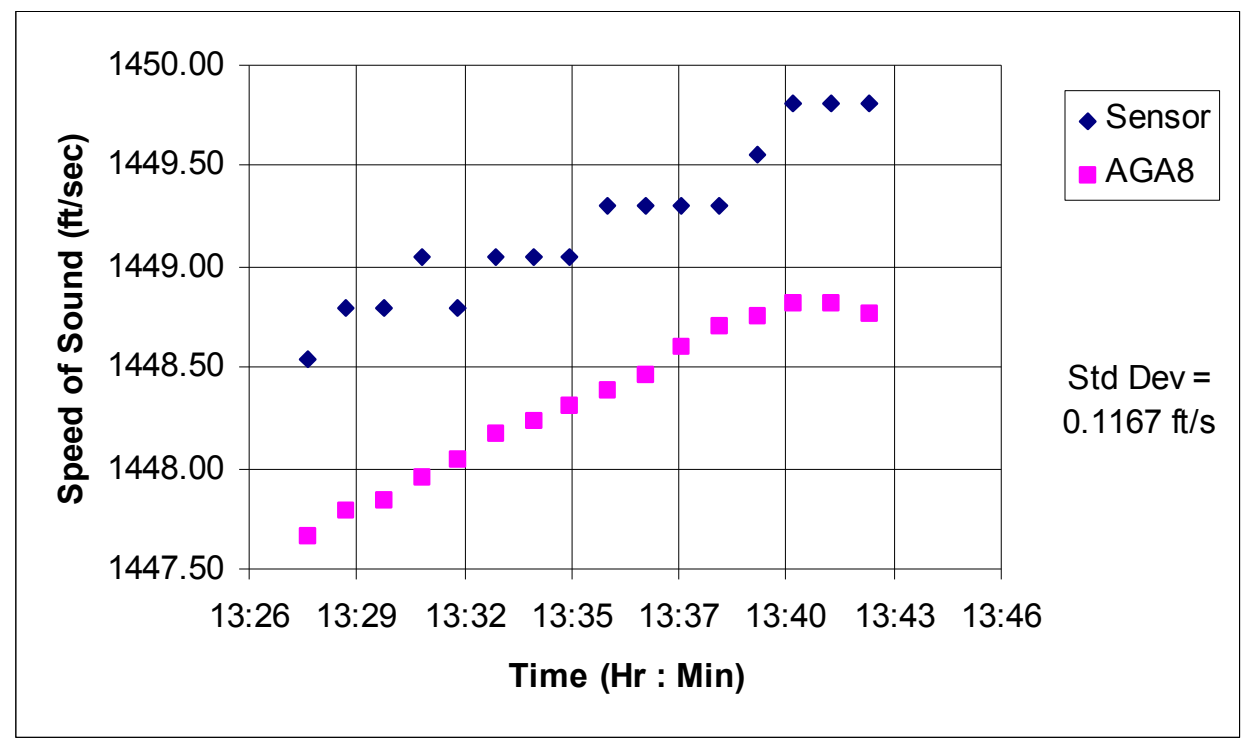

Figure 3.29. HPL 8/21/01 run \#1 $\left(P_{a v}=98.3\right.$ psia, $\left.T_{a v}=96.1^{\circ} F\right)$.

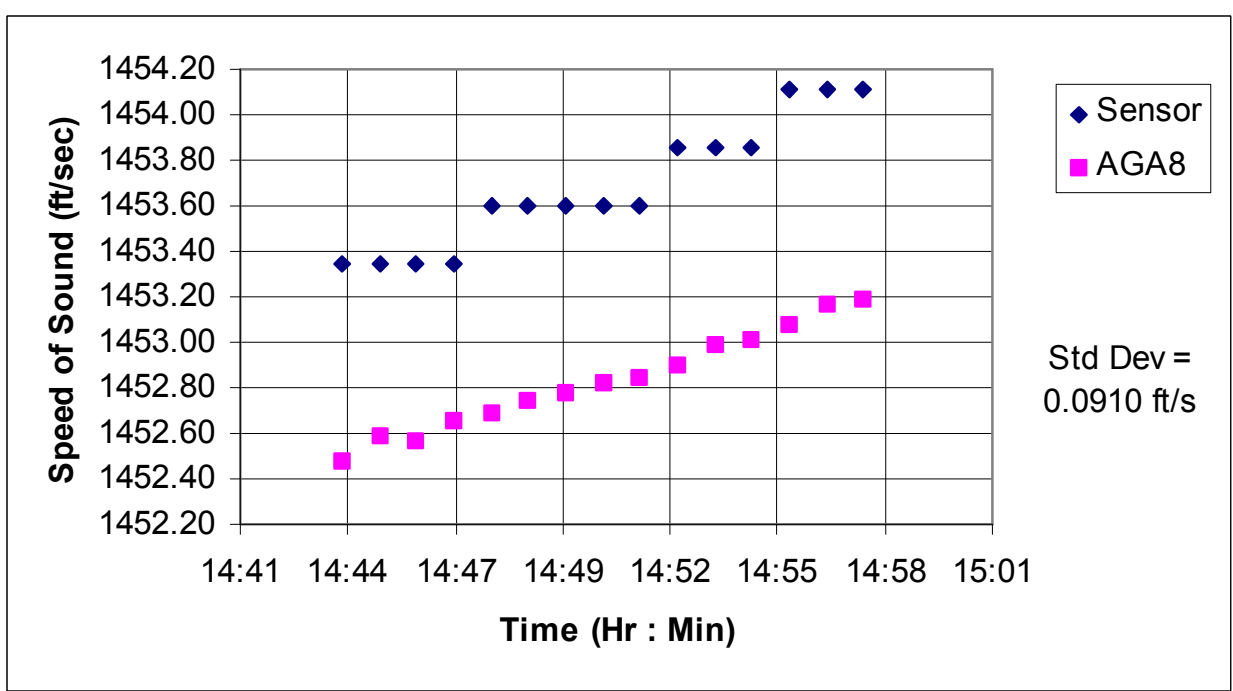

Figure 3.30. $\mathrm{HPL} 8 / 21 / 01$ run \#2 $\left(\mathrm{P}_{\mathrm{av}}=98.7 \mathrm{psia}, \mathrm{T}_{\mathrm{av}}=99.8^{\circ} \mathrm{F}\right)$. 


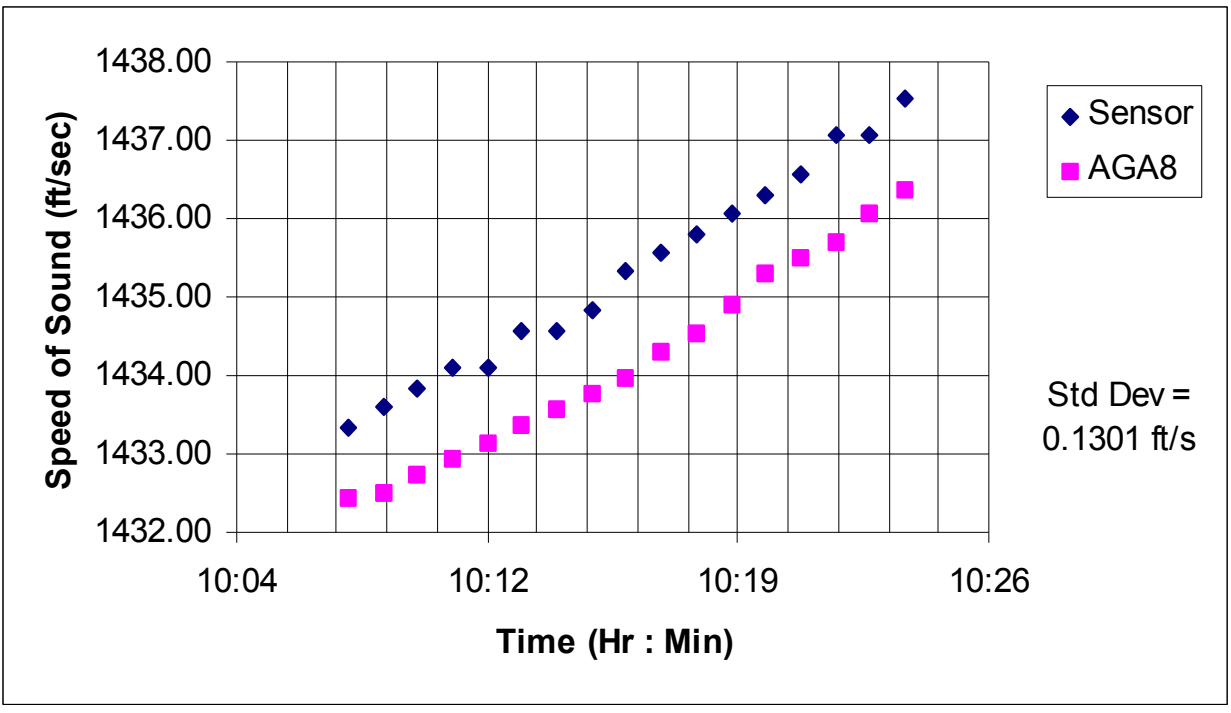

Figure 3.31. $\mathrm{HPL} \mathrm{8/22/01}$ run \#2 $\left(\mathrm{P}_{\mathrm{av}}=98.3 \mathrm{psia}, \mathrm{T}_{\mathrm{av}}=84.4^{\circ} \mathrm{F}\right)$.

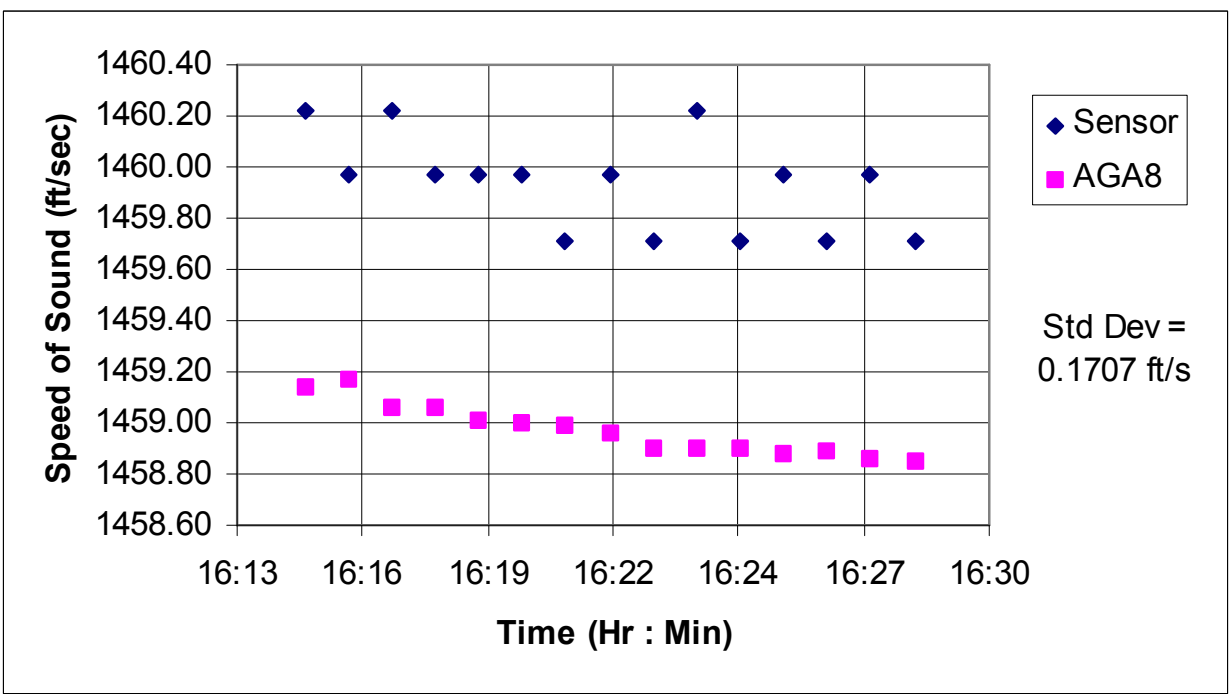

Figure 3.32. $\mathrm{HPL} 8 / 22 / 01$ run \#7 $\left(\mathrm{P}_{\mathrm{av}}=95.0 \mathrm{psia}, \mathrm{T}_{\mathrm{av}}=104.5^{\circ} \mathrm{F}\right)$. 


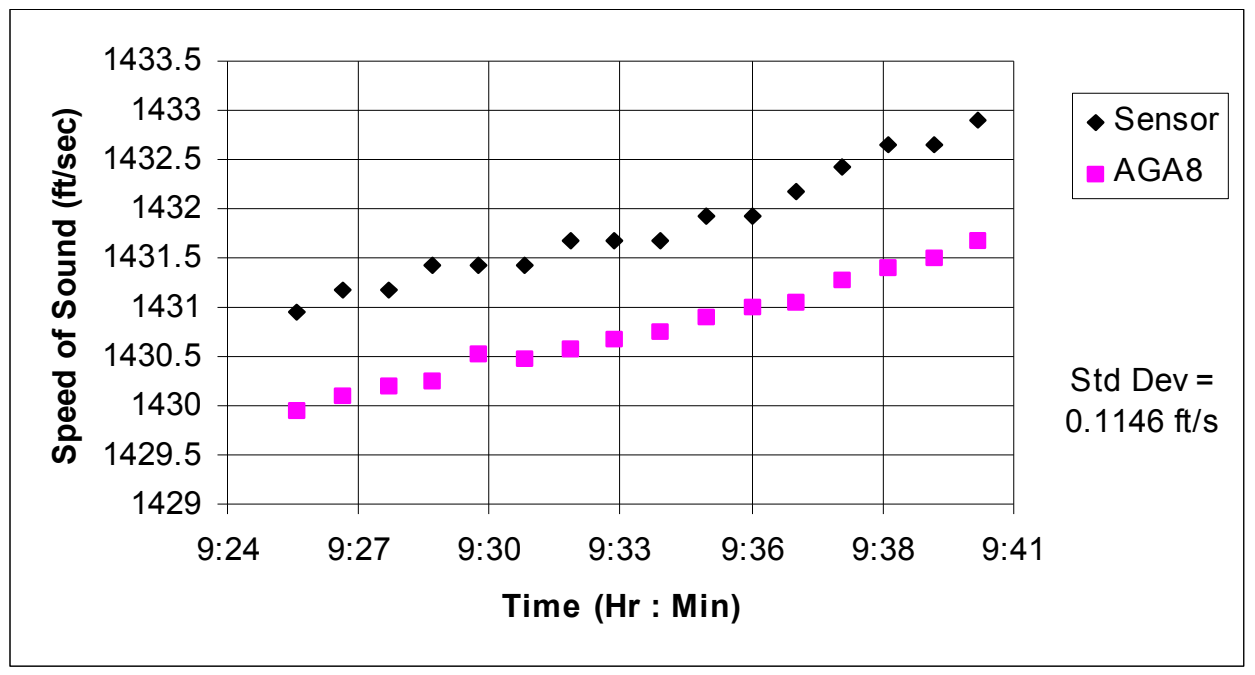

Figure 3.33. HPL 8/23/01 run \#2 $\left(P_{a v}=99.7 p s i a, T_{a v}=81.8^{\circ} \mathrm{F}\right)$.

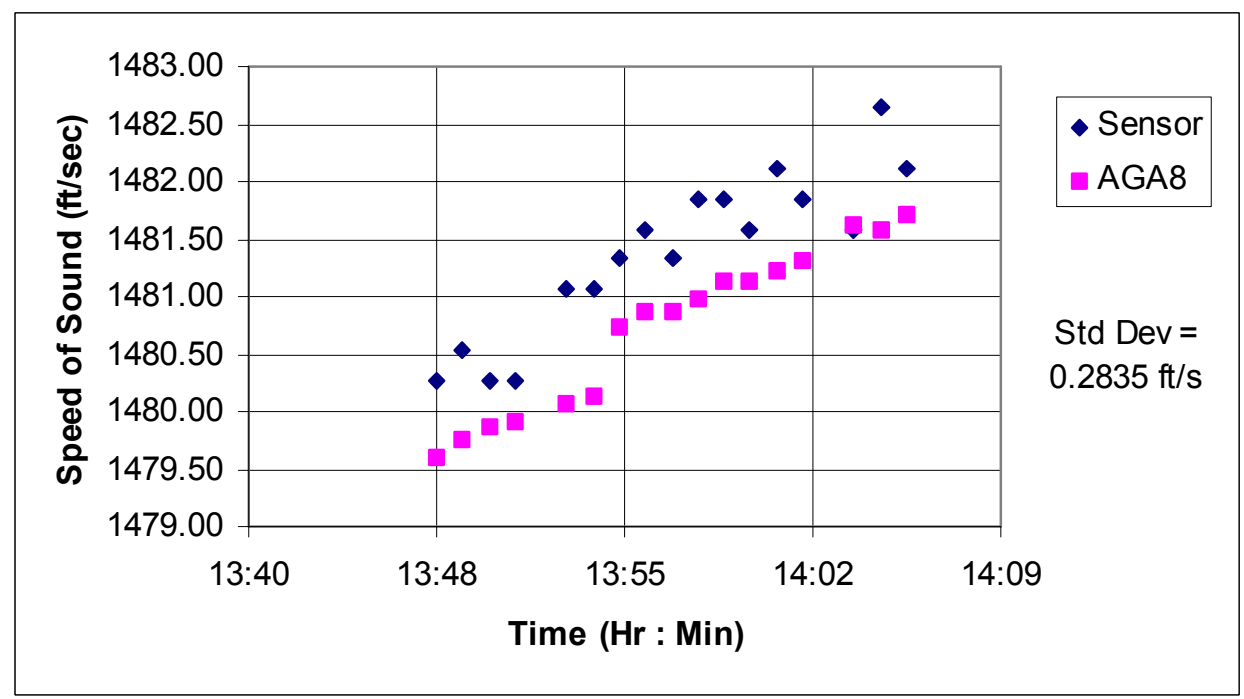

Figure 3.34. $\mathrm{HPL} 8 / 23 / 01$ run \#7 $\left(\mathrm{P}_{\mathrm{av}}=92.7 \mathrm{psia}, \mathrm{T}_{\mathrm{av}}=122.6^{\circ} \mathrm{F}\right)$. 


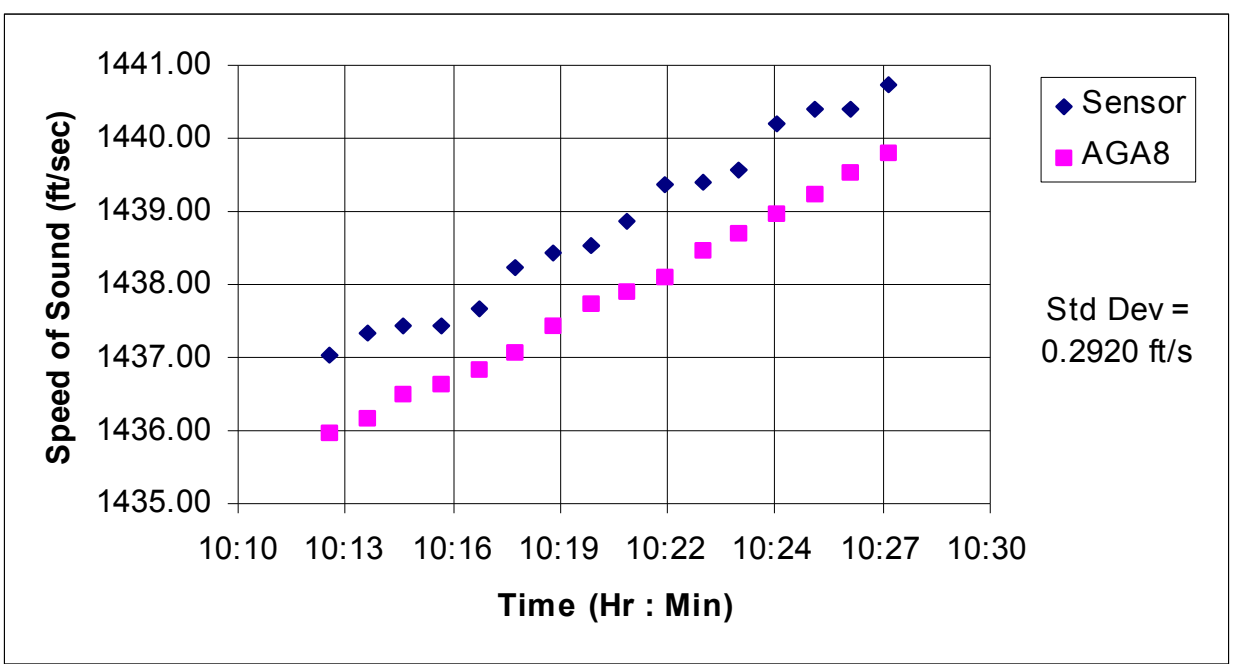

Figure 3.35. HPL 8/24/01 run \#2 $\left(P_{a v}=96.3\right.$ psia, $\left.T_{a v}=87.5^{\circ} \mathrm{F}\right)$.

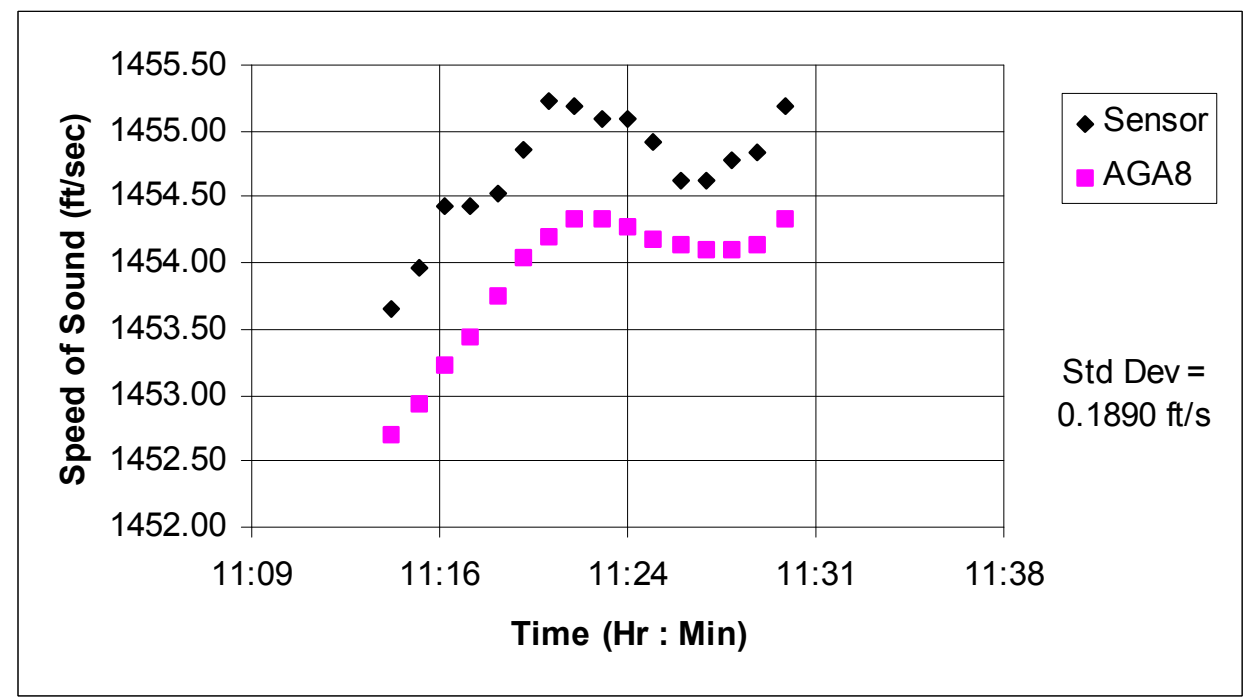

Figure 3.36. $\mathrm{HPL} \mathrm{8/24/01}$ run \#4 $\left(\mathrm{P}_{\mathrm{av}}=95.2 \mathrm{psia}, \mathrm{T}_{\mathrm{av}}=100.9^{\circ} \mathrm{F}\right)$. 
Table 3.5. Gas composition used in LPL laboratory tests of energy module.

\begin{tabular}{|l|c|}
\hline Gas Constituent & $\begin{array}{c}\text { Composition } \\
\text { (mol\%) }\end{array}$ \\
\hline Methane & 96.4120 \\
\hline Ethane & 1.4365 \\
\hline Carbon Dioxide & 1.17279 \\
\hline Nitrogen & 0.83904 \\
\hline Propane & 0.09503 \\
\hline Isobutane & 0.01050 \\
\hline n-Butane & 0.01491 \\
\hline Isopentane & 0.00480 \\
\hline n-Pentane & 0.00380 \\
\hline n-Hexane & 0.00349 \\
\hline n-Heptane & 0.00358 \\
\hline n-Octane & 0.00235 \\
\hline n-Nonane & 0.00024 \\
\hline
\end{tabular}

Figure 3.37 is an example of the ultrasonic pulse waveforms, frequency spectra, and correlation response indicating the time-of-flight difference between the two reflected pulses. These data were recorded during the third day of the four-day testing sequence and are representative of the ultrasonic signals observed during all of the higher pressure $(\sim 100 \mathrm{psia})$ tests in the LPL test runs. The waveforms recorded at the lower pressure ( $\sim 50 \mathrm{psia})$ were lower in signal-to-noise ratio, causing a wider range of scatter in the measured values of speed of sound. The ultrasonic transducer used in the energy meter was the Etalon Model CIA-3525-SB2 described earlier.

Figures 3.38 through 3.43 present test results from six LPL runs, selected from a total of 17 runs, to illustrate the accuracy and response time of the speed-of-sound sensor. Most of these data showed a positive offset between the experimental measurements and the calculated values of speed of sound ranging from about $0.25 \mathrm{ft} / \mathrm{s}$ to about $2.9 \mathrm{ft} / \mathrm{s}$. Part of this error was accounted for by the thermal sensitivity of the sensor $\left(3.389 \times 10^{-3} /{ }^{\circ} \mathrm{F}\right)$, and the remainder was attributed to velocity dispersion in the gas mixture and other possible sources of error in the energy meter module. The temperature range of the measurements was less than in the HPL tests because the module was shaded from direct sunlight and several test days were overcast. The primary variable in all of the tests was the composition of the gas and, in particular, the addition of $\mathrm{CO}_{2}$. To change the $\mathrm{CO}_{2}$ content, normal natural gas was doped with $\mathrm{CO}_{2}$ during flow circulation until the $\mathrm{CO}_{2}$ concentration was near the target value. Thus, each test run had a somewhat different gas mixture. The same speed-of-sound sensor calibration results described in Section 3.2.7 for the HPL tests (one week earlier) were used in the LPL tests. 
In general, the experimental measurements in the LPL tests were more scattered than those observed in the HPL tests. This is believed to be due to fluctuations in the composition of the natural gas; as it circulated in the loop and was doped with $\mathrm{CO}_{2}$, brief local variations in composition may have occurred until the carbon dioxide was evenly distributed in the loop. The scatter may also be due to differences in the locations of the GC sample probes and the speed-ofsound measurements between the LPL and HPL. Figure 3.38 shows the speed of sound measured in normal natural gas first at a pressure of 107 psia and followed by measurements at a pressure of $51 \mathrm{psia}$. The high-pressure values had a $0.25 \mathrm{ft} / \mathrm{s}$ positive offset relative to the AGA-8 calculated values. The experimental values measured at 50 psia had a relatively large amount of scatter, which masked the presence of any offset. The scatter was caused by a low signal-to-noise ratio in the ultrasonic pulse waveforms at reduced pressure. The offset errors in the data were somewhat less than those observed in the HPL tests, and since the normal gas compositions were very similar, there was no obvious explanation for the difference.

Figure 3.39 shows test runs at $49.1 \mathrm{psia}$ and $107.5 \mathrm{psia}$ with the $\mathrm{CO}_{2}$ content increased to $9.3 \mathrm{~mol} \%$. The offset errors in these runs were higher by about $2.5 \mathrm{ft} / \mathrm{s}$ and $2.0 \mathrm{ft} / \mathrm{s}$, respectively, as might be expected from velocity dispersion effects in a higher $\mathrm{CO}_{2}$ and lower methane gas. These measurements also contain a larger amount of scatter.

Figures 3.40 and 3.41 show test runs at 109.9 psia and 50.8 psia with the $\mathrm{CO}_{2}$ content adjusted to approximately $3.2 \mathrm{~mol} \%$. The higher pressure data were offset by $2.9 \mathrm{ft} / \mathrm{s}$ with low scatter, and the lower pressure data were offset by $0.6 \mathrm{ft} / \mathrm{sec}$ with substantial scatter. These offsets, although larger than those observed in the normal gas in Figure 3.38, followed the same trend, namely, a smaller offset under the lower pressure condition.

Figures 3.42 and 3.43 show test runs at 49.9 psia and 107.5 psia with the $\mathrm{CO}_{2}$ content adjusted to approximately $5.8 \mathrm{~mol} \%$. The higher pressure data were offset by $2.3 \mathrm{ft} / \mathrm{sec}$ with low scatter, and the lower pressure data were offset by $1.0 \mathrm{ft} / \mathrm{s}$ with low scatter. These offsets also followed the trends observed in the normal gas in Figure 3.38, namely, a smaller offset under the lower pressure condition. However, the amount of offset in these measurements at $5.8 \mathrm{~mol} \%$ $\mathrm{CO}_{2}$ appeared to be inconsistent with those observed for the 9.3 and the $3.2 \mathrm{~mol} \% \mathrm{CO}_{2}$ concentrations. The explanation for these differences is likely to be associated with the more complex velocity dispersion effects in gas mixtures containing larger amounts of $\mathrm{CO}_{2}$ and would require test results that focus on dispersion effects to be resolved. 

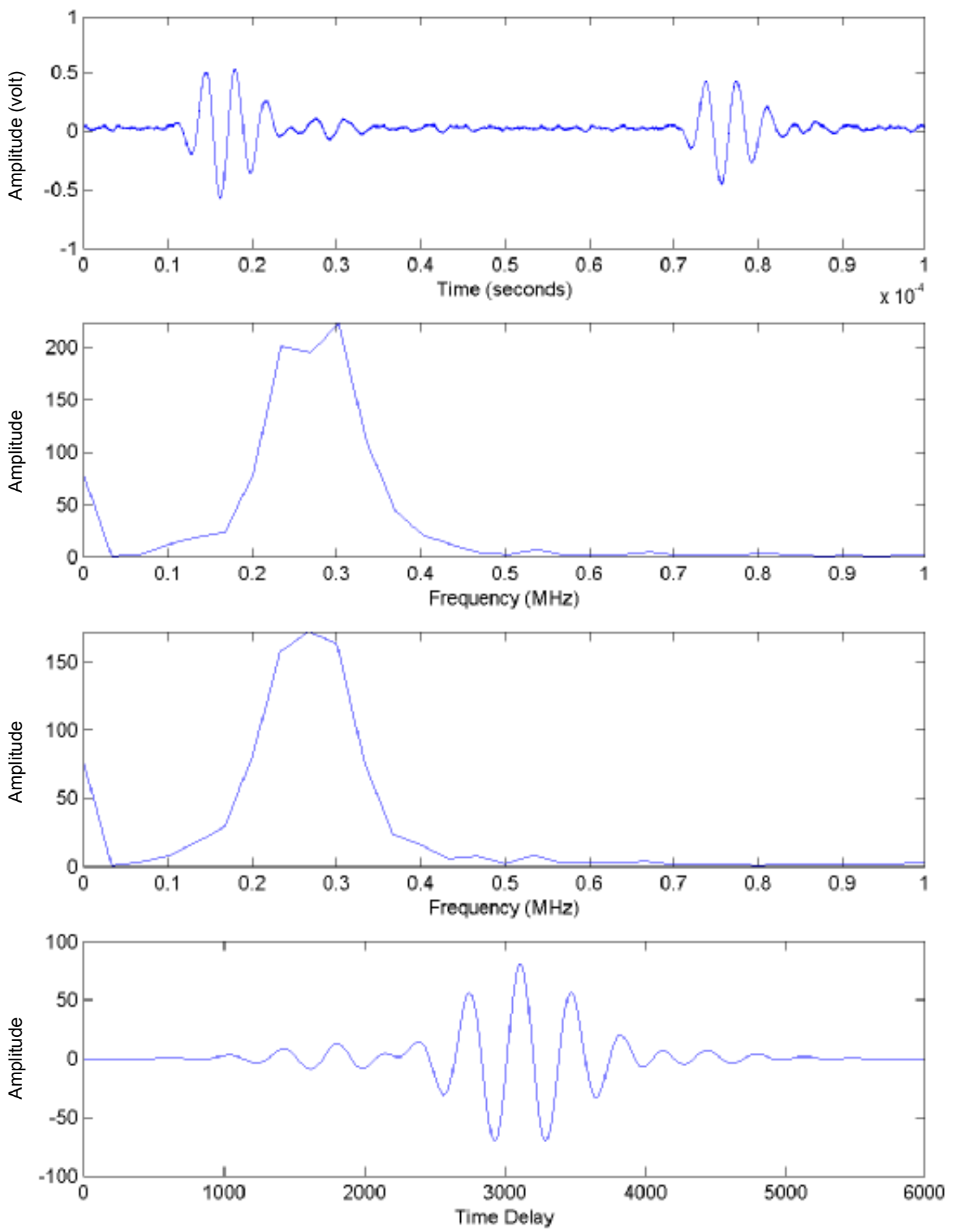

Figure 3.37. Pulse signals, frequency spectra, and cross-correlation function: Etalon CIA-3525-SB2 266-kHz ultrasonic transducer in MRF LPL gas.

Pressure: 107.35 psia; temperature: $96.13^{\circ} \mathrm{F}$. 


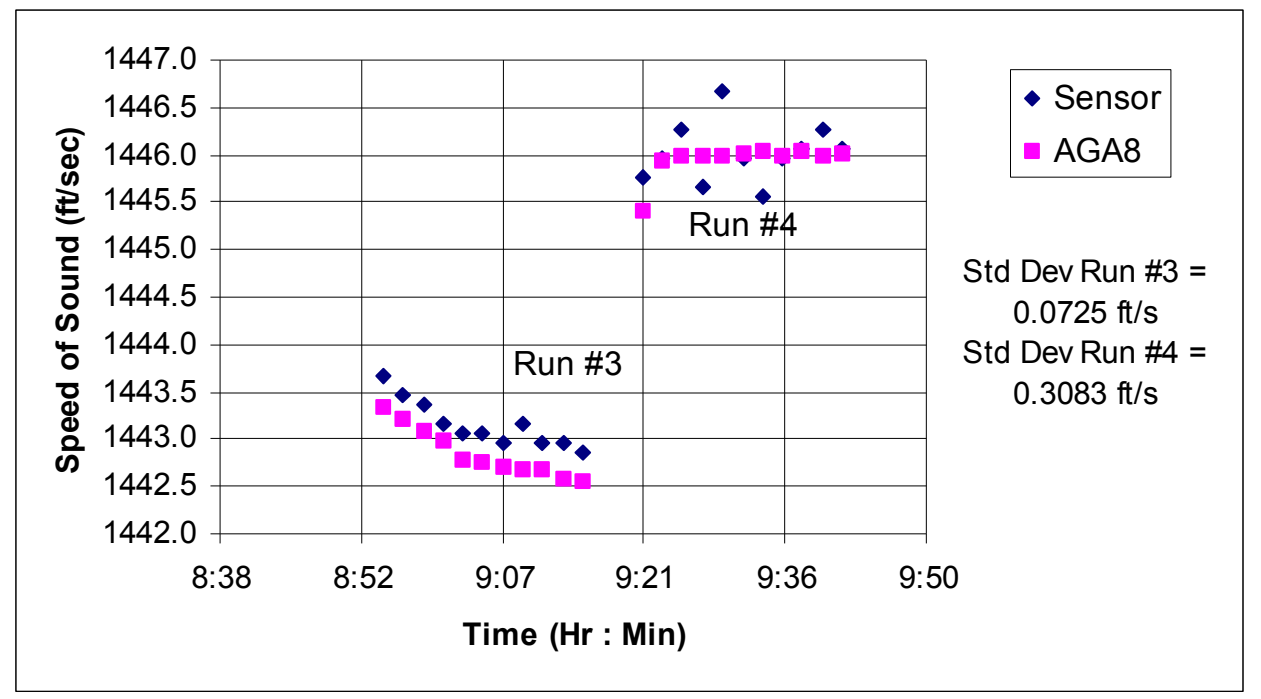

Figure 3.38. LPL 9/7/01 run \#3 $\left(P_{a v}=107 \mathrm{psia}, T_{a v}=84^{\circ} \mathrm{F}, \mathrm{CO}_{2 \mathrm{av}}=1.158 \mathrm{~mol} \%\right)$ and run \#4 $\left(\mathrm{P}_{\mathrm{av}}=51 \mathrm{psia}, \mathrm{T}_{\mathrm{av}}=84.1^{\circ} \mathrm{F}, \mathrm{CO}_{2 \mathrm{av}}=1.157 \mathrm{~mol} \%\right)$.

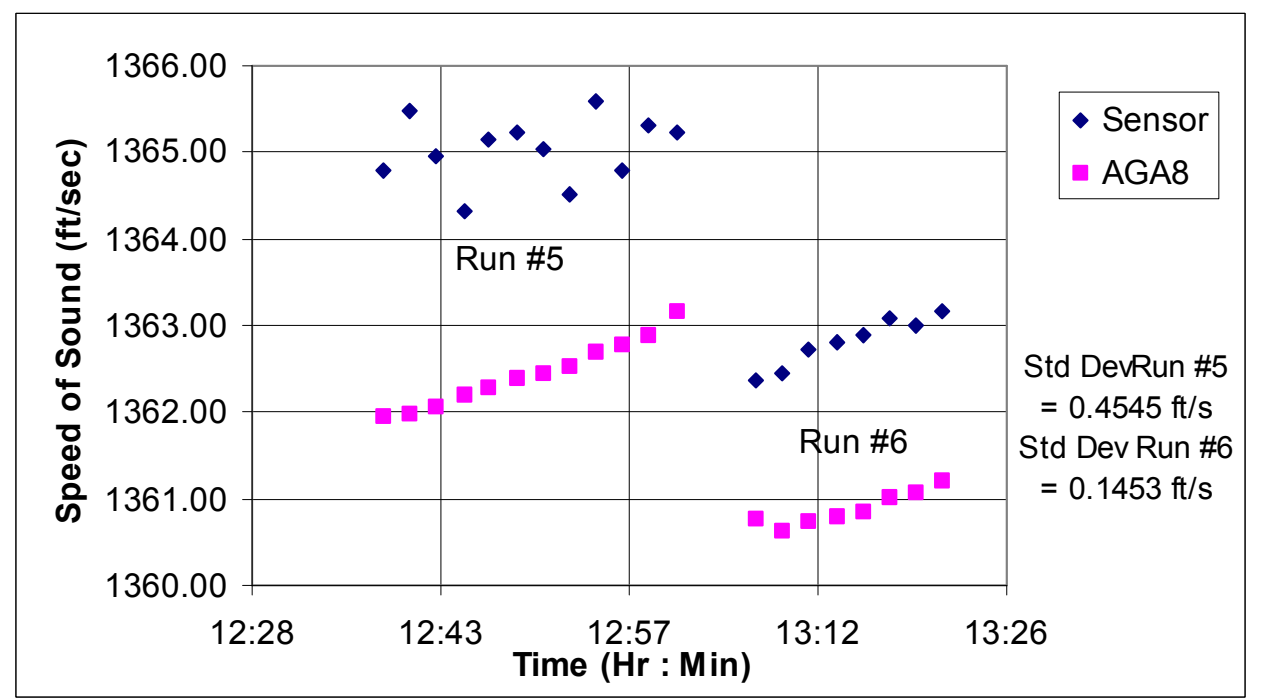

Figure 3.39. LPL $9 / 7 / 01$ run \#5 $\left(P_{a v}=49.1 \mathrm{psia}, \mathrm{T}_{\mathrm{av}}=89.3^{\circ} \mathrm{F}, \mathrm{CO}_{2 \mathrm{av}}=9.379 \mathrm{~mol} \%\right)$ and run \#6 $\left(P_{a v}=107.5\right.$ psia, $\left.\mathrm{T}_{\mathrm{av}}=90.6^{\circ} \mathrm{F}, \mathrm{CO}_{2 \mathrm{av}}=9.281 \mathrm{~mol} \%\right)$. 


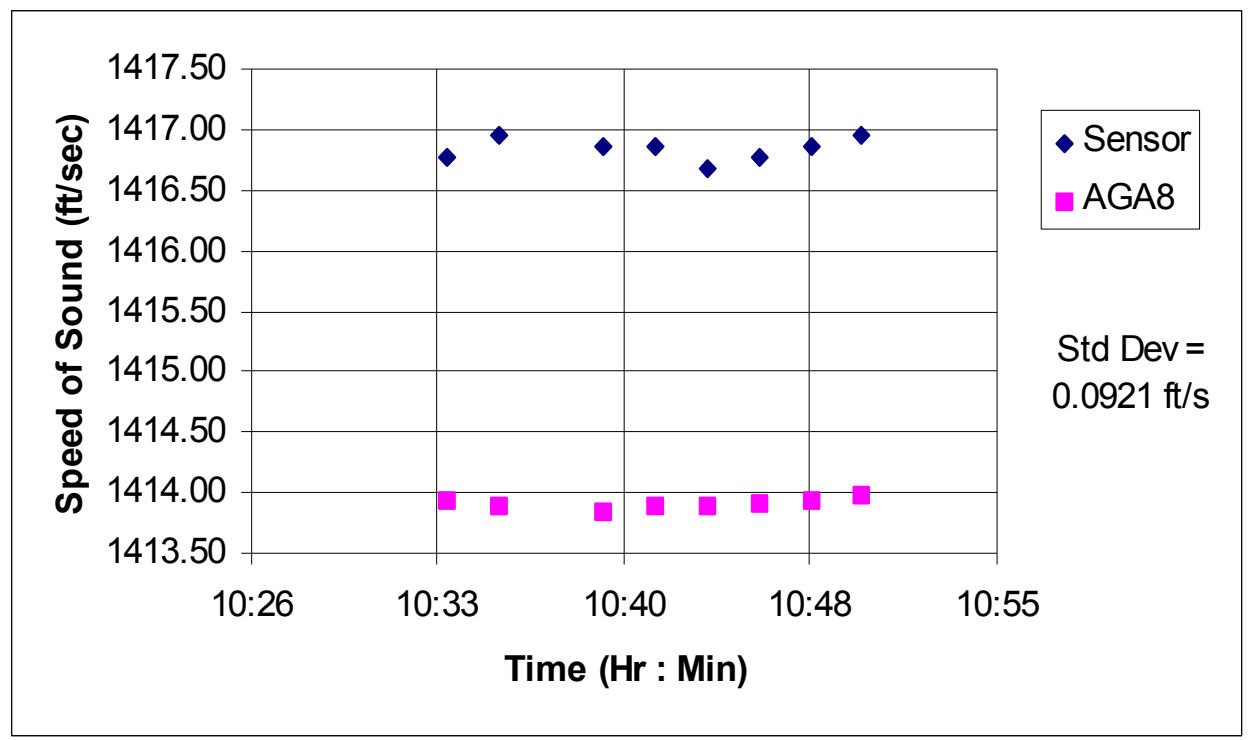

Figure 3.40. LPL 9/10/01 run \#2 $\left(P_{a v}=109.9\right.$ psia, $\left.T_{a v}=81.1^{\circ} \mathrm{F}, \mathrm{CO}_{2 \mathrm{av}}=3.35 \mathrm{~mol} \%\right)$.

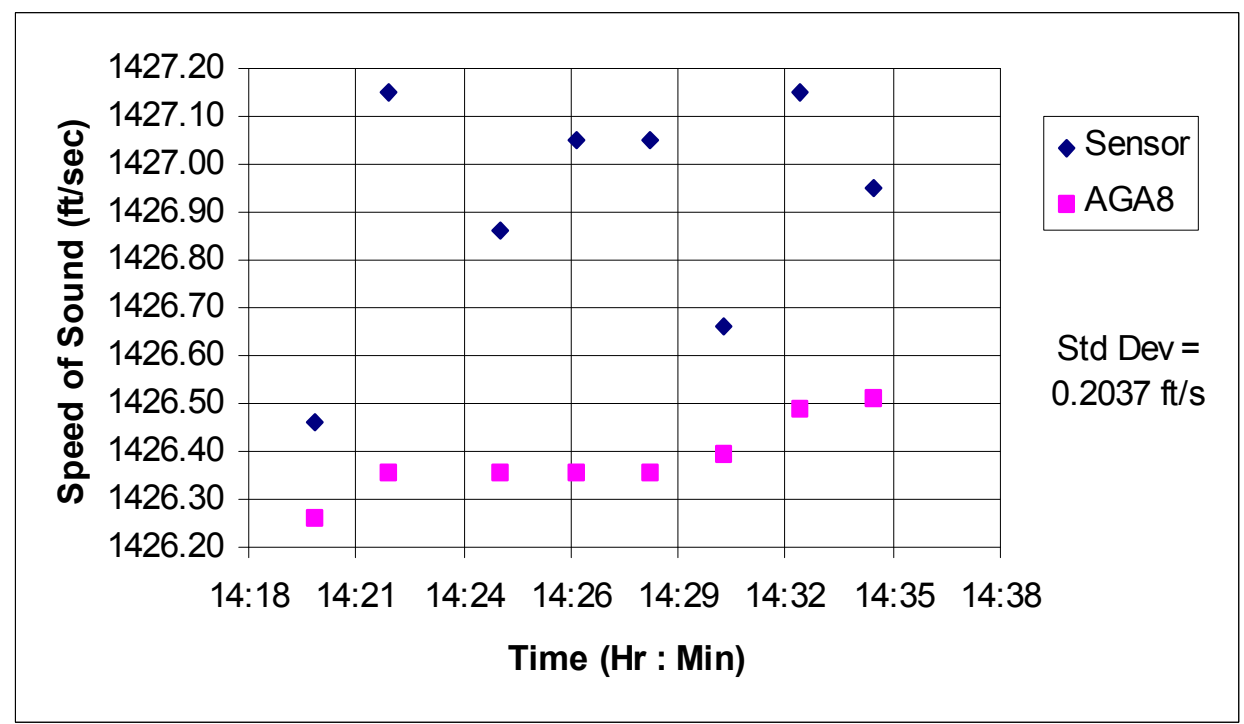

Figure 3.41. $\mathrm{LPL} 9 / 10 / 01 \mathrm{run} \# 5\left(\mathrm{P}_{\mathrm{av}}=50.8 \mathrm{psia}, \mathrm{T}_{\mathrm{av}}=84.9^{\circ} \mathrm{F}, \mathrm{CO}_{2 \mathrm{av}}=3.050 \mathrm{~mol} \%\right)$. 


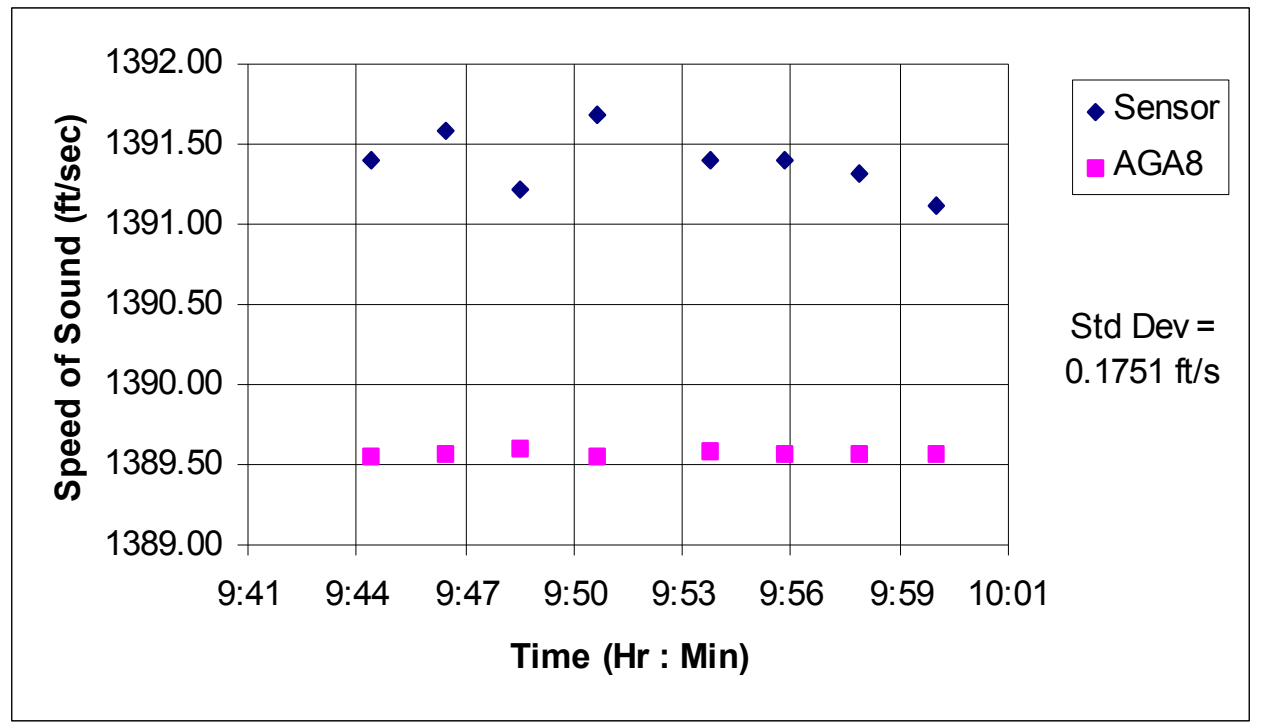

Figure 3.42. LPL 9/11/01 run \#1 $\left(P_{a v}=49.9\right.$ psia, $\left.T_{a v}=81.8^{\circ} \mathrm{F}, C_{2} O_{a v}=6.063 \mathrm{~mol} \%\right)$.

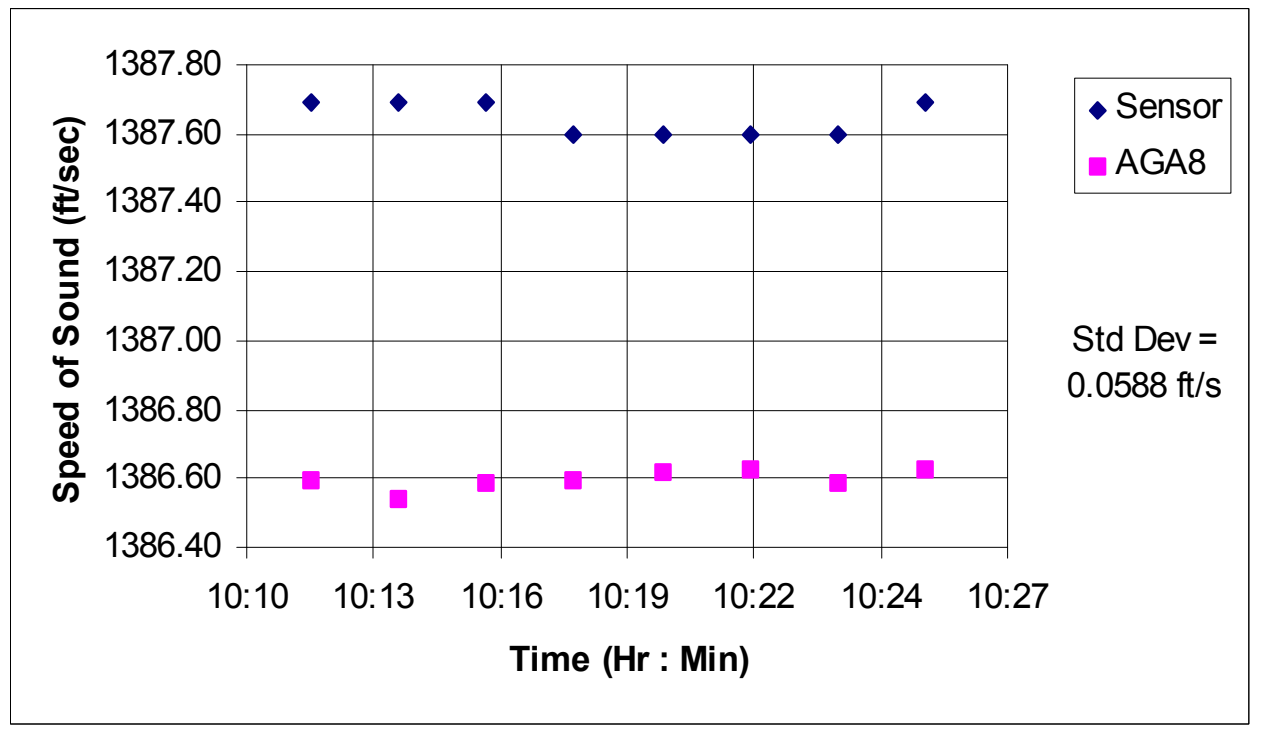

Figure 3.43. $L P L 9 / 11 / 01$ run \#2 $\left(P_{a v}=107.5\right.$ psia, $\left.T_{a v}=81.9^{\circ} \mathrm{F}, C_{2} a v=5.97 \mathrm{~mol} \%\right)$. 


\section{3 $\quad \mathrm{CO}_{2}$ Sensors and Performance Testing}

\subsubsection{Background}

Carbon dioxide is an important inert diluent affecting the energy density of natural gas and, hence, its custody transfer pricing. The $\mathrm{CO}_{2}$ content in transmission-quality natural gas typically ranges from less than $1 \mathrm{~mol} \%$ up to about $3 \mathrm{~mol} \%$. The upper limit is an industry regulatory constraint aimed at maintaining a minimum gas quality standard and minimizing pipeline corrosion. To be widely applicable in natural gas transmission and distribution systems, $\mathrm{CO}_{2}$ sensors are needed that will operate in outdoor pipeline field environments at typical line pressures ranging from about $50 \mathrm{psi}$ to $1400 \mathrm{psi}$, without the intermediate step of reducing the pipeline gas to atmospheric pressure for measurement. Additionally, the sensor must either be intrinsically safe or made explosion-proof for approved use in hazardous industrial environments.

To date, commercial $\mathrm{CO}_{2}$ sensor technology has not been developed for such stringent applications in natural gas transmission/distribution systems. However, as an important step toward this goal, volume fraction measurements of $\mathrm{CO}_{2}$ in natural gas were recently demonstrated with promising success as part of the 1999-2000 efforts of this project [Morrow et al. (2000)]. Those preliminary tests were conducted using commercially available sensors designed for $\mathrm{CO}_{2}$ measurements in air or in certain industrial processes at or near one atmosphere pressure. The sensing method is based on optical absorption effects that are unique to $\mathrm{CO}_{2}$ when infrared radiation is transmitted through a short column of the gas mixture under test. Since the hydrocarbon constituents of natural gas do not exhibit the same optical absorption bands as $\mathrm{CO}_{2}$, the sensing method was equally applicable to measurements in natural gas. Present-day $\mathrm{CO}_{2}$ sensors are designed to operate at or near atmospheric pressure. They are typically calibrated at reference conditions of 1 atm and $77^{\circ} \mathrm{F}$, and their readings may be compensated for typical atmospheric variations from these conditions using independent linear scaling factors.

Additional tests of this $\mathrm{CO}_{2}$ sensing technology were performed during the period covered in this report with the primary goal of determining the inherent accuracy and resolution of the method, as presently implemented in commercially available sensing devices, when measuring $\mathrm{CO}_{2}$ in natural gas. These tests confirmed the practicability of the infrared absorption sensing technique to meet the demanding measurement requirements of the natural gas energy meter. These more extensive sensor studies have demonstrated that, for natural gas samples having $\mathrm{CO}_{2}$ levels of 1 to $2 \mathrm{~mol} \%$, the $\mathrm{CO}_{2}$ content was measurable to within about $\pm 2 \%$ of its actual value, which is well within the accuracy required for deriving the gas heating value within $\pm 1 \mathrm{Btu} / \mathrm{scf}$.

However, tests on natural gas doped with $\mathrm{CO}_{2}$ to levels of 3,6 , and $9 \mathrm{~mol} \%$ indicated that the sensor response was nonlinear with errors approaching +7 to $+10 \%$ of the actual $\mathrm{CO}_{2}$ content. Further evaluation of this sensor nonlinearity will be required to develop a quantitative compensation method for linearizing the sensor based on interdependent parameters of pressure, temperature, and $\mathrm{CO}_{2}$ content. 


\subsection{2 $\mathrm{CO}_{2}$ Measurement Method}

The measurement method used in most commercial $\mathrm{CO}_{2}$ sensors is based on infrared optical absorption by $\mathrm{CO}_{2}$ molecules along a short path between an infrared source and an infrared detector. The source is a miniature incandescent lamp capable of producing stable optical radiation in the $1-5 \mu \mathrm{m}$ near-infrared wavelength range. The detector is a semiconductor photodiode responsive to near-infrared radiation. To achieve useful detection sensitivity and to ensure that only $\mathrm{CO}_{2}$ absorption is measured, a narrowband optical interference filter designed to match the near-infrared $\mathrm{CO}_{2}$ absorption wavelength of $4.26 \mu \mathrm{m}$ is used as the detector optical window. The intensity of the infrared radiation at this wavelength is reduced in proportion to the path length and, more specifically, to the number of $\mathrm{CO}_{2}$ molecules in the optical path through the gas. Therefore, the sensing method is a phenomenon that occurs at the molecular level rather than as a bulk effect in the gas. The photodiode detector output is fundamentally a measure of the mole fraction $\mathrm{CO}_{2}$ content in the gas under test.

The single-optical-path measurement technique described above is adequate for many industrial $\mathrm{CO}_{2}$ measurement applications. However, it has limitations that can affect measurement accuracy, resolution, and long-term calibration stability. The limitations are related to changes in source radiation intensity, contaminants accumulated on the optical windows, and aging of the photodiode detector. Improved designs have been developed to overcome the problems associated with variations in source intensity by using a single source and two optical transmission and detection paths, one having an interference filter tuned to the $4.26 \mu \mathrm{m} \mathrm{CO}_{2}$ absorption band and one tuned to an adjacent wavelength range where no infrared absorption occurs. The non-absorbing channel provides a reference response unrelated to the $\mathrm{CO}_{2}$ content but directly related to the source intensity and, to a useful degree, to any common build-up of contamination on the optical windows. Nevertheless, this two-channel system remains subject to changes and mismatched conditions in the two photodiode detectors as well as to nonuniform contamination conditions on the optical windows.

A new and improved sensing arrangement patented and manufactured by the Vaisala Corporation of Finland is capable of minimizing these problems. This sensor, patented and registered under the trade name Carbocap ${ }^{\circledR}$ [Koshinen et al. (1977)], is a single-path design having an electrically tunable optical interference filter that is alternately adjusted to the $4.26 \mu \mathrm{m}$ $\mathrm{CO}_{2}$ absorption wavelength and an adjacent wavelength where no absorption occurs. In this case, the non-absorbing response serves as a reference measurement by which the three sources of error mentioned above may be accurately compensated. During the past year, sensors of the single-path, dual-path, and the Carbocap ${ }^{\circledR}$ type were acquired for testing, with emphasis on the Carbocap $^{\circledR}$ device because of its expected better accuracy.

\subsection{3 $\mathrm{CO}_{2}$ Sensor Technical Specifications}

Three infrared absorption $\mathrm{CO}_{2}$ sensors were purchased for testing and evaluation on this project: (1) a Vaisala Model GMM12B (0-3 mol\% full-scale single-path sensor); (2) a Texas Instruments Model 9GS-4 (0-5 mol\% full-scale dual-path sensor); and (3) a Vaisala Model GMP221B (0-10 mol\% full-scale Carbocap ${ }^{\circledR}$ sensor). Table 3.6 summarizes the primary technical specifications of these sensors. 
Table 3.6. Carbon dioxide sensor technical specifications.

\begin{tabular}{|c|c|c|c|}
\hline $\begin{array}{l}\text { TECHNICAL } \\
\text { SPECIFICATION }\end{array}$ & $\begin{array}{c}\text { Vaisala } \\
\text { Model GMM12B }\end{array}$ & $\begin{array}{l}\text { Texas Instruments } \\
\text { Model 9GS-4 }\end{array}$ & $\begin{array}{c}\text { Vaisala } \\
\text { Model GMP221B }\end{array}$ \\
\hline Sensor Design & $\begin{array}{l}\text { Basic single-path } \\
\text { infrared absorption } \\
\text { sensor ( } 4.26 \mu \mathrm{m} \\
\text { wavelength) }\end{array}$ & $\begin{array}{l}\text { Dual-path infrared } \\
\text { absorption sensor } \\
\text { with second lamp } \\
\text { reference channel } \\
(4.26 \mu \mathrm{m})\end{array}$ & $\begin{array}{l}\text { Carbocap }^{\circledR} \text { infrared } \\
\text { absorption sensor } \\
\text { (electrically tunable IR } \\
\text { interference filter at } \\
3.8 \mu \mathrm{m} \text { and } 4.26 \mu \mathrm{m} \text { ) }\end{array}$ \\
\hline Measurement Range & $0-3 \mathrm{~mol} \% \mathrm{CO}_{2}$ & $0-5 \mathrm{~mol} \% \mathrm{CO}_{2}$ & $0-10 \mathrm{~mol} \% \mathrm{CO}_{2}$ \\
\hline Measurement Accuracy & $\pm 0.09 \mathrm{~mol} \% \mathrm{CO}_{2}$ & $\pm 0.25 \mathrm{~mol} \% \mathrm{CO}_{2}$ & $\pm 0.05 \mathrm{~mol} \% \mathrm{CO}_{2}$ \\
\hline Pressure Effect & $\mathrm{N} / \mathrm{A}$ & $\begin{array}{l}9.80 \times 10^{-3} \text { times } \\
\text { sensor reading } / p s i\end{array}$ & $\begin{array}{l}10.32 \times 10^{-3} \text { times } \\
\text { sensor reading } / p s i\end{array}$ \\
\hline Temperature Effect & $-0.009 \mathrm{~mol} \% /{ }^{\circ} \mathrm{F}$ & N/A & $-0.009 \mathrm{~mol} \%{ }^{\circ} \mathrm{F}$ \\
\hline Response Time & $\begin{array}{l}30 \text { sec to } 63 \% \text { of } \\
\text { step change in } \mathrm{CO}_{2}\end{array}$ & $\begin{array}{l}<20 \text { sec to } 63 \% \text { of } \\
\text { step change in } \mathrm{CO}_{2}\end{array}$ & $\begin{array}{l}30 \text { sec to } 63 \% \text { of step } \\
\text { change in } \mathrm{CO}_{2}\end{array}$ \\
\hline Output Signal & $\begin{array}{l}\text { 4-20 ma : zero to } \\
\text { FS }\end{array}$ & 4-20 ma : zero to FS & 4-20 ma : zero to FS \\
\hline Supply Voltage & $18-30 \mathrm{VDC}$ & $12 \mathrm{VDC} \pm 5 \%$ & 18-30 VDC \\
\hline Temperature Rating & $\begin{array}{l}0 \text { to } 45^{\circ} \mathrm{C} \text { (opr) } \\
-30 \text { to } 70^{\circ} \mathrm{C} \text { (sto) }\end{array}$ & $\begin{array}{l}0 \text { to } 50^{\circ} \mathrm{C} \text { (opr) } \\
-40 \text { to } 70^{\circ} \mathrm{C} \text { (sto) }\end{array}$ & $\begin{array}{l}-20 \text { to } 60^{\circ} \mathrm{C} \text { (opr) } \\
-30 \text { to } 70^{\circ} \mathrm{C} \text { (sto) }\end{array}$ \\
\hline Humidity Rating & N/A & $5-95 \%$ (non-cond) & 0-85\% (non-cond) \\
\hline Intrinsically Safe & No & No & No \\
\hline
\end{tabular}

Figures 3.44 through 3.46 show photographs of the three $\mathrm{CO}_{2}$ sensing modules. The Vaisala Model GMM12B sensor in Figure 3.44 is an uncased module in which the gas test cell has been separated from the electronics circuit board for remote operation in a controlled gas mixture environment within a small pressure vessel. Electrical connections between the gas test cell and the circuit board are made through a multi-circuit electrical feed-through header in the cover plate of the pressure vessel. With this arrangement, any necessary control adjustments of test measurements of the sensor can be made during controlled pressure and temperature test runs. The Texas Instruments Model 9GS-4 sensor in Figure 3.45 is also an uncased module with its gas test cell removed for remote operation in the pressure vessel. To maintain the integrity of this sensor configuration, the test cell is mounted on a small auxiliary printed circuit board to permit proper placement and orientation of the infrared source lamp. The Vaisala Model GMP221B sensor in Figure 3.46 employs a hand-held gas test probe connected to the electronic unit by a multiconductor cable. To test this sensor in the pressure vessel, the cable was parted and the leads connected through the electrical feed-through header. 


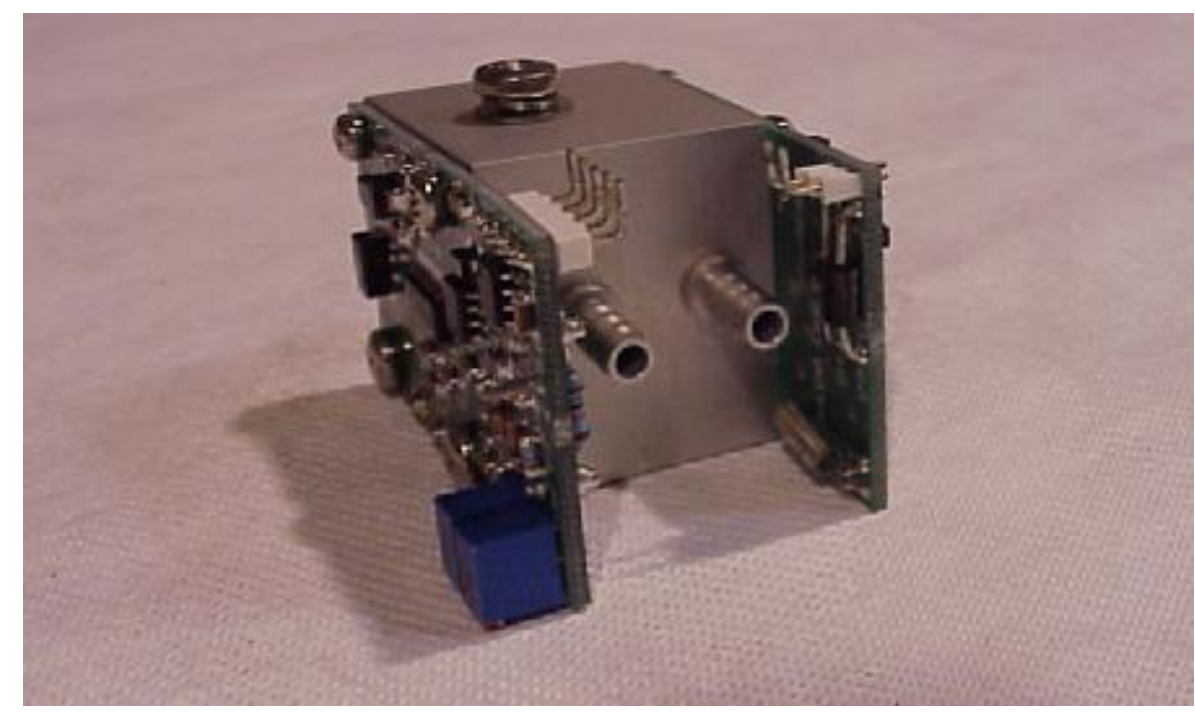

Figure 3.44. Photograph of Vaisala $\mathrm{CO}_{2}$ sensor Model GMM12B.

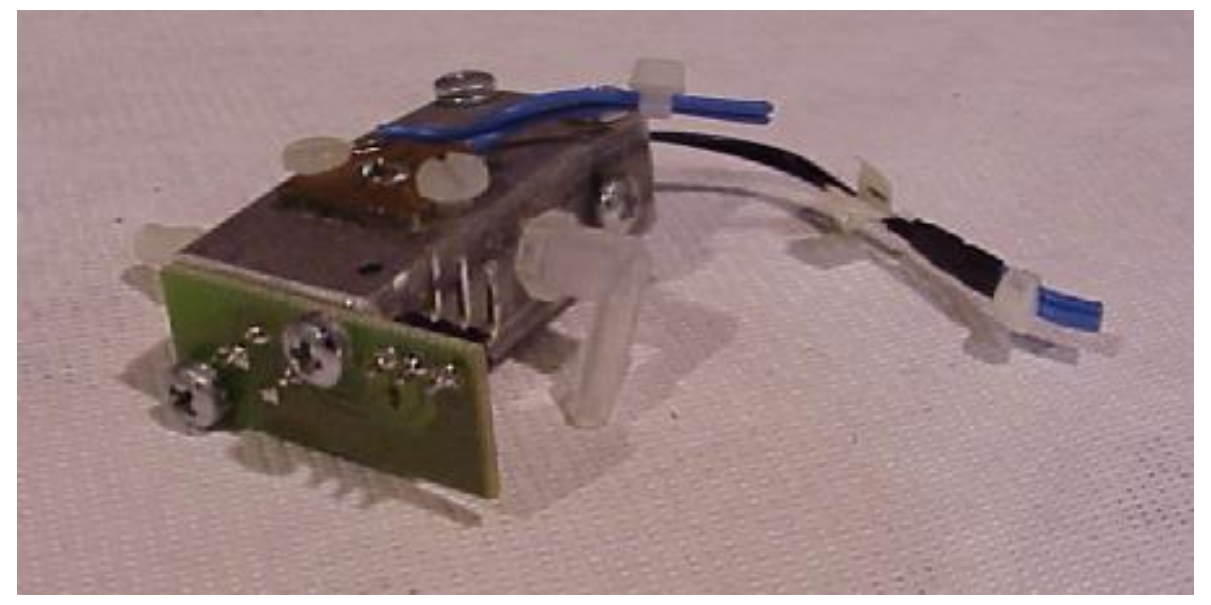

Figure 3.45. Photograph of uncased Texas Instruments $\mathrm{CO}_{2}$ sensor.

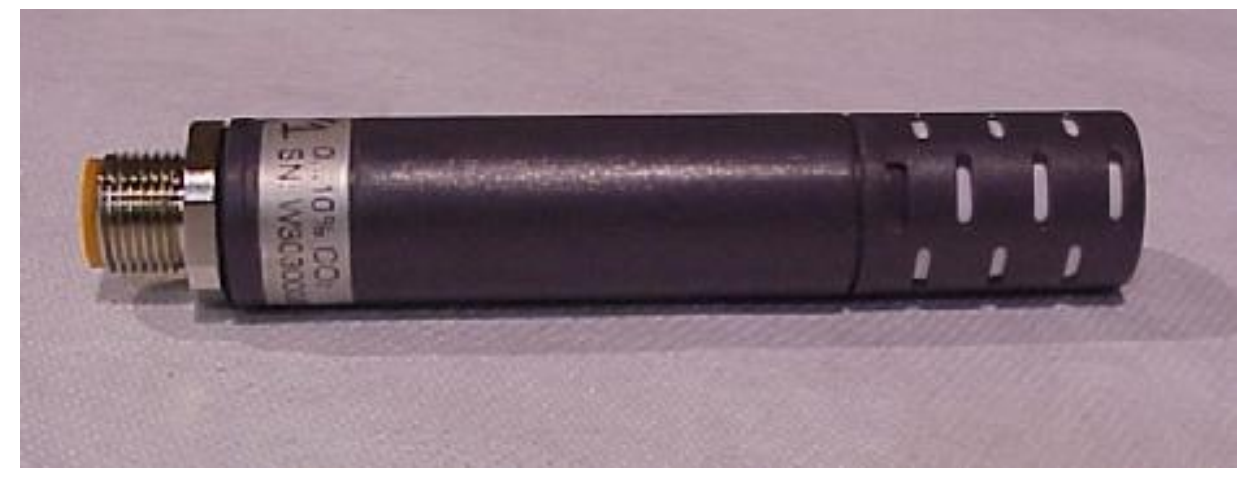

Figures 3.46. Photograph of Vaisala $\mathrm{CO}_{2}$ sensor Model GMP221B. 


\subsection{4 $\mathrm{CO}_{2}$ Sensor Test Considerations and Baseline Test}

Although comprehensive testing of the three $\mathrm{CO}_{2}$ sensors was not within the scope of the present project, a limited effort was applied to prepare for such tests in the future. This work consisted of bench setup tests of the three sensors in a pressure vessel to determine their basic operating performance and to establish their baseline temperature sensitivity in a dry "zero air" environment ("zero air" composition: 80.5-76.5 mol\% $\mathrm{N}_{2} ; 19.5-23.5 \mathrm{~mol} \% \mathrm{O}_{2} ;<3 \mathrm{ppm} \mathrm{CO}$; $<3$ ppm $\mathrm{H}_{2} \mathrm{O} ;<1 \mathrm{ppm}$ THC) at temperatures in the $75-145^{\circ} \mathrm{F}$ range. In order to determine the inherent temperature effects on the gas test cells alone in the absence of any $\mathrm{CO}_{2}$ absorption effects, the sensor electronic circuit boards were not exposed to elevated temperatures. Figure 3.47 shows the laboratory setup for these tests using a $1.5 \mathrm{ft}^{3}$ pressure vessel on loan to SwRI from the U.S. Department of Commerce (NIST). The test results showed that the baseline output signals from the three sensors readily responded to small but arbitrary amounts of $\mathrm{CO}_{2}$ introduced into the vessel but were essentially unaffected by elevated temperature exposure in "zero air."

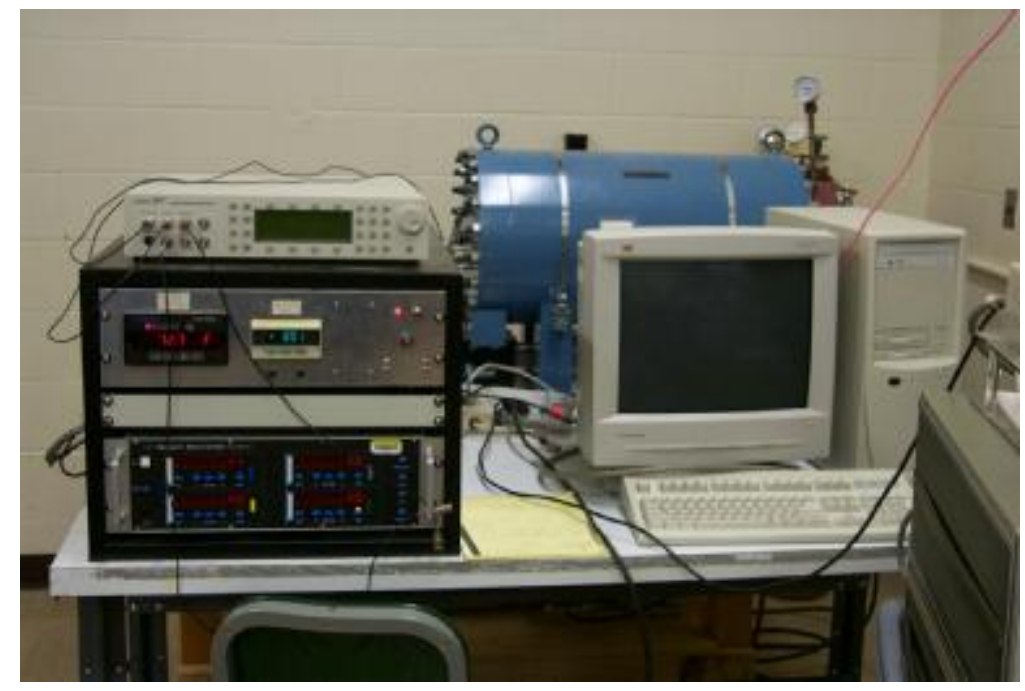

Figure 3.47. Photograph of pressure vessel setup.

Technical considerations for evaluating $\mathrm{CO}_{2}$ sensors for use in natural gas pipelines are concerned with the combined effects of the gas pressure and temperature on the accuracy and resolution of the sensor. The infrared absorption process occurs at the molecular level and, therefore, is not expected to be influenced in a fundamental way by the gas pressure or temperature. That is, while the width of the infrared absorption band will become somewhat broader with increasing pressure, the optical wavelength at which maximum absorption occurs is not expected to change. Thus, it will not be necessary to change the narrowband interference filters used in the sensor when operating at different gas pressures. However, since the absorption increases with the increase in gas density, for a given $\mathrm{CO}_{2}$ concentration the infrared energy reaching the detector will be reduced as the pressure increases. In this case, whereas long optical path lengths are necessary for accurate measurements of relatively low $\mathrm{CO}_{2}$ concentrations at one atmosphere, the path length must be reduced to sense similar concentrations at elevated 
pressures. Even shorter path lengths may be required for measuring higher $\mathrm{CO}_{2}$ concentrations at higher pressures, depending upon the realizable dynamic range of the infrared sensor. Therefore, a $\mathrm{CO}_{2}$ sensor designed for the $0-6 \mathrm{~mol} \%$ range may be required to have more than one optical path length or sensor channel in order to accommodate different operating pressure ranges.

In addition to optical path considerations, the $\mathrm{CO}_{2}$ sensor operating pressure and temperature must be known so that proper calibration corrections can be applied to obtain accurate $\mathrm{CO}_{2}$ measurements. Present-day $\mathrm{CO}_{2}$ sensors operating at one atmosphere pressure and typical seasonal variations in temperature require only independent linear temperature and pressure corrections to achieve their intended measurement accuracy (typically about $\pm 2 \%$ of reading). However, accuracy requirements for measuring $\mathrm{CO}_{2}$ concentrations in the inferential gas energy meter application are much more stringent (typically about $\pm 0.05 \mathrm{~mol} \%$ absolute; e.g., less than $\pm 0.83 \%$ at $6 \mathrm{~mol} \%$ reading) over a wide range of pressures and temperatures. Therefore, in addition to long-term sensor stability, the sensor calibration process required for precision $\mathrm{CO}_{2}$ measurements in natural gas will very likely entail interdependent and nonlinear pressure and temperature corrections.

\subsection{5 $\mathrm{CO}_{2}$ Measurement Chamber for Energy Meter Module}

The Vaisala Model GMP221B $\mathrm{CO}_{2}$ sensor was selected for use in the prototype energy meter module because of its superior technical specifications and advanced compensation design. This sensor and a specialized $\mathrm{CO}_{2}$ test chamber were assembled as part of the energy meter module. The 0.73 -in. diameter infrared absorption probe is mounted in one end of a cylindrical test chamber similar to that used for the speed-of-sound measurements. This chamber is instrumented to measure the internal gas pressure, temperature, and throughput volume flow rate in the immediate vicinity of the sensor probe. Figure 3.48 shows the design details of the $\mathrm{CO}_{2}$ measurement chamber. The natural gas entering the $\mathrm{CO}_{2}$ measurement chamber is the output gas from the speed-of-sound sensor chamber reduced in pressure from a value in the 50-100 psia range to approximately 15 psia by a fine-control needle valve. Before entering the measurement chamber the gas travels through a covered helical groove machined in the outer surface of the aluminum $\mathrm{CO}_{2}$ test chamber body to allow the gas to equilibrate to the temperature of the metallic chamber. At the outlet end of the helical path, the gas enters an annular anterior space formed by the inner wall of the aluminum cylinder and a 0.10 -in. thick porous coaxial cylindrical sleeve formed of expanded sintered aluminum. This porous sleeve serves as a diffusion interface to prevent the formation of any persistent gas flow patterns in the chamber. The $\mathrm{CO}_{2}$ sensor probe passes through one end of the chamber and is sealed in place by O-rings. The outlet port at the other end of the chamber is connected to a rotameter gas flow sensor and control valve through which the gas exits to the atmosphere at a typical release rate of approximately 0.20 $1 / \mathrm{min}$.

As a result of the regulated gas pressure in the speed-of-sound sensor chamber and the controlled pressure reduction and low flow rate through the $\mathrm{CO}_{2}$ test chamber, the test gas conditions at the $\mathrm{CO}_{2}$ sensor module are maintained stable and uniform for the measurements. Pressure and temperature ports are also located on the outlet end of the test chamber for connecting a Rosemount Model 3051 0-30 psia pressure sensor and inserting a 0.1-in. diameter stainless-steel-jacketed RTD probe into the central zone of the chamber. The aluminum body has an outside diameter of 2.75-in. and a metal wall thickness of 0.125-in. A helical groove 0.094-in. wide and 0.062-in. deep is machined to provide a gas flow length of $200 \mathrm{in}$. and a typical flow 
dwell time of $6 \mathrm{sec}$ at the flow rate of $0.20 \mathrm{l} / \mathrm{min}$ before entering the chamber. A close-tolerance cylindrical cover sleeve made of Black Almagon ${ }^{\circledR}$ graphite-epoxy slides over the groove and is sealed at the end plates by O-rings. One end of the chamber contains the access ports for the gas pressure and temperature sensors. The overall length of the chamber is $5.125 \mathrm{in}$. The thermal mass of the aluminum chamber acts as a heat sink to the gas under test but undergoes relatively slow changes in temperature in response to changes in ambient conditions within the energy meter module. The temperature and pressure of the gas in the $\mathrm{CO}_{2}$ test chamber are accurately measured and are used to correct the $\mathrm{CO}_{2}$ sensor readings for variations relative to the standard sensor calibration reference values of $77^{\circ} \mathrm{F}$ and 14.73 psia. Figure 3.49 is a cutaway drawing of the $\mathrm{CO}_{2}$ test chamber showing the helical groove and the Vaisala sensor probe in place.

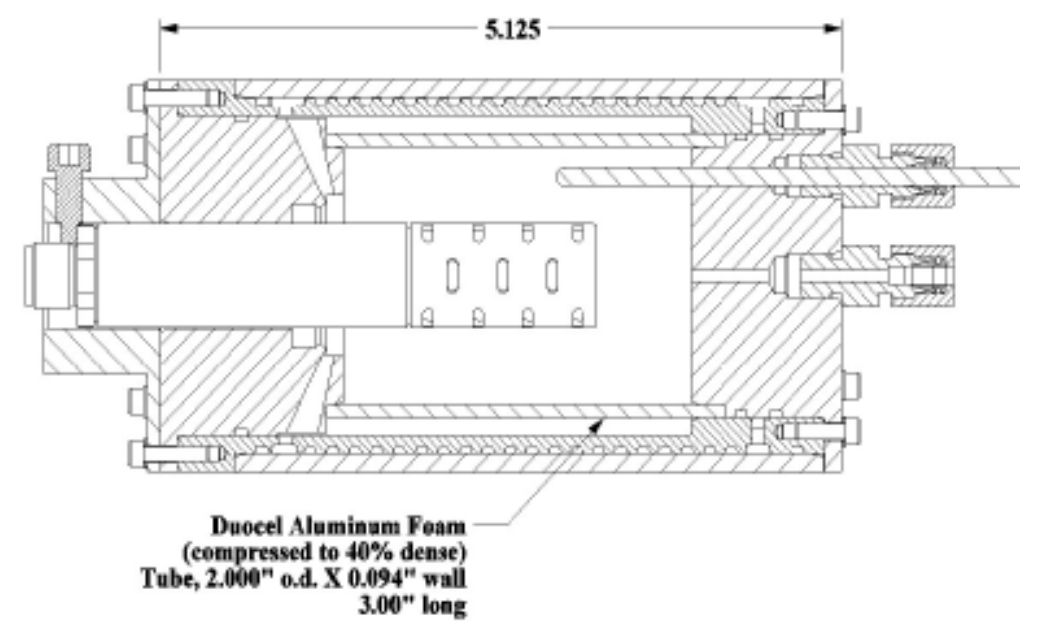

Figure 3.48. Layout drawing of $\mathrm{CO}_{2}$ test chamber.

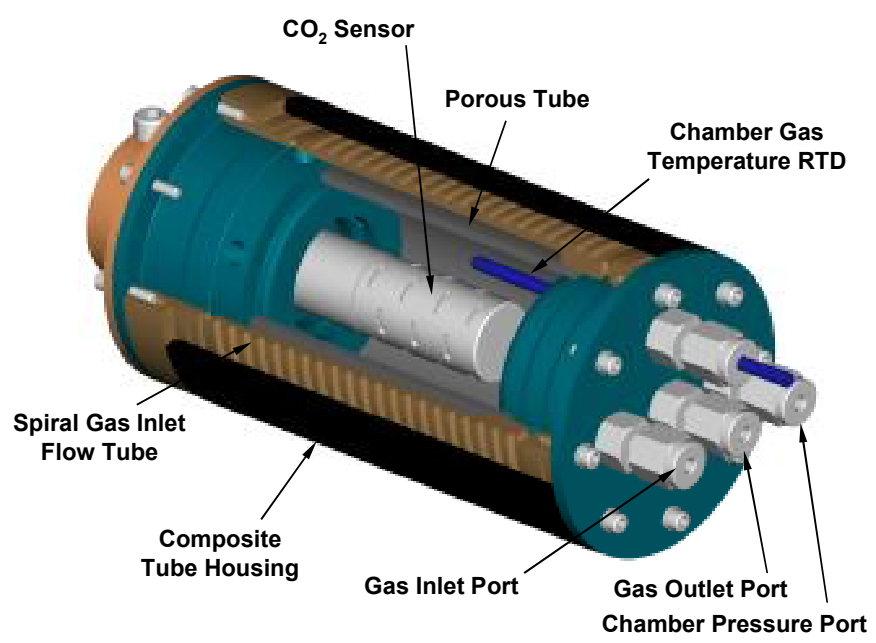

Figure 3.49. Cutaway drawing of $\mathrm{CO}_{2}$ test chamber. 


\subsection{6 $\mathrm{CO}_{2}$ Sensor Measurements in Natural Gas}

Controlled flow-loop tests were conducted to evaluate the prototype energy meter module containing the Vaisala Model GMP221B $\mathrm{CO}_{2}$ sensor operating with samples of commercial natural gas and similar gas samples containing added amounts of $\mathrm{CO}_{2}$. In these tests, gas from the flow loop was tapped via a manually adjusted pressure regulator valve for use in the energy meter module and the $\mathrm{CO}_{2}$ measurement chamber. Gas chromatograph data were recorded periodically during the various flow loop tests to provide reference information on the gas composition. The $\mathrm{CO}_{2}$ content in the normal natural gas samples was typically in the range of 1-2 mol\% and, in some of the test runs, the normal gas was doped with dry $\mathrm{CO}_{2}$ to raise the concentration to approximately 3,6 , and $9 \mathrm{~mol} \%$.

Data related to the $\mathrm{CO}_{2}$ sensor measurements during each flow loop test run included:

1. Test run time (date, hour, minute, second)

2. Gas temperature in $\mathrm{CO}_{2}$ test chamber $\left({ }^{\circ} \mathrm{F}\right)$

3. Gas pressure in $\mathrm{CO}_{2}$ test chamber (psia)

4. $\mathrm{CO}_{2}$ sensor output current (4-20 ma for $0-10 \mathrm{~mol} \%$ full scale)

5. Gas exit flow rate out of the $\mathrm{CO}_{2}$ test chamber was maintained constant at $0.20 \mathrm{l} / \mathrm{min}$ by needle valve adjustment

6. MRF gas chromatograph readings of flow loop gas concentrations

\subsubsection{High-Pressure Loop Tests}

Tests performed in the MRF HPL involved natural gas having $\mathrm{CO}_{2}$ content in the range of $1.35-1.37 \mathrm{~mol} \%$. The ambient temperatures in the energy meter module during the various test runs ranged from about $78^{\circ} \mathrm{F}$ during early morning tests to about $125^{\circ} \mathrm{F}$ during midafternoon tests. Data related to $\mathrm{CO}_{2}$ measurements were recorded during approximately 26 hours over a four-day period. Although the gas temperature, pressure, and flow rate were changed for testing purposes, the gas composition remained essentially unchanged throughout all of the HPL tests.

Figures 3.50 through 3.53 show the reduced $\mathrm{CO}_{2}$ measurement results for HPL test runs conducted on August 21-24, 2001. In all of these figures the MRF GC $\mathrm{CO}_{2}$ values are shown by the heavy lines and are assumed to be accurate reference values representing the $\mathrm{CO}_{2}$ content in the flow loop gas. The thin lines define the upper and lower tolerance bounds $( \pm 0.05 \mathrm{~mol} \% \mathrm{ref}$ $\mathrm{GC}$ value) in which the $\mathrm{CO}_{2}$ sensor measurements must fall in order to yield gas heating values within an accuracy of $\pm 1 \mathrm{Btu} / \mathrm{scf}$. The data points show the temperature- and pressure-corrected $\mathrm{CO}_{2}$ sensor measurements recorded at two-minute intervals during the test runs. The measured $\mathrm{CO}_{2}$ mean values (medium weight lines) derived from the data points were within $0.01 \mathrm{~mol} \%$ of the $\mathrm{GC}$ values. The variations in measured $\mathrm{CO}_{2}$ content were within about $\pm 0.015 \mathrm{~mol} \%$ throughout several hours of data logging during the four days of testing. For the ambient operating conditions that existed during these tests, the technical performance of the Vaisala GMP221B $\mathrm{CO}_{2}$ sensor was sufficiently accurate and stable for use in the energy meter application. 


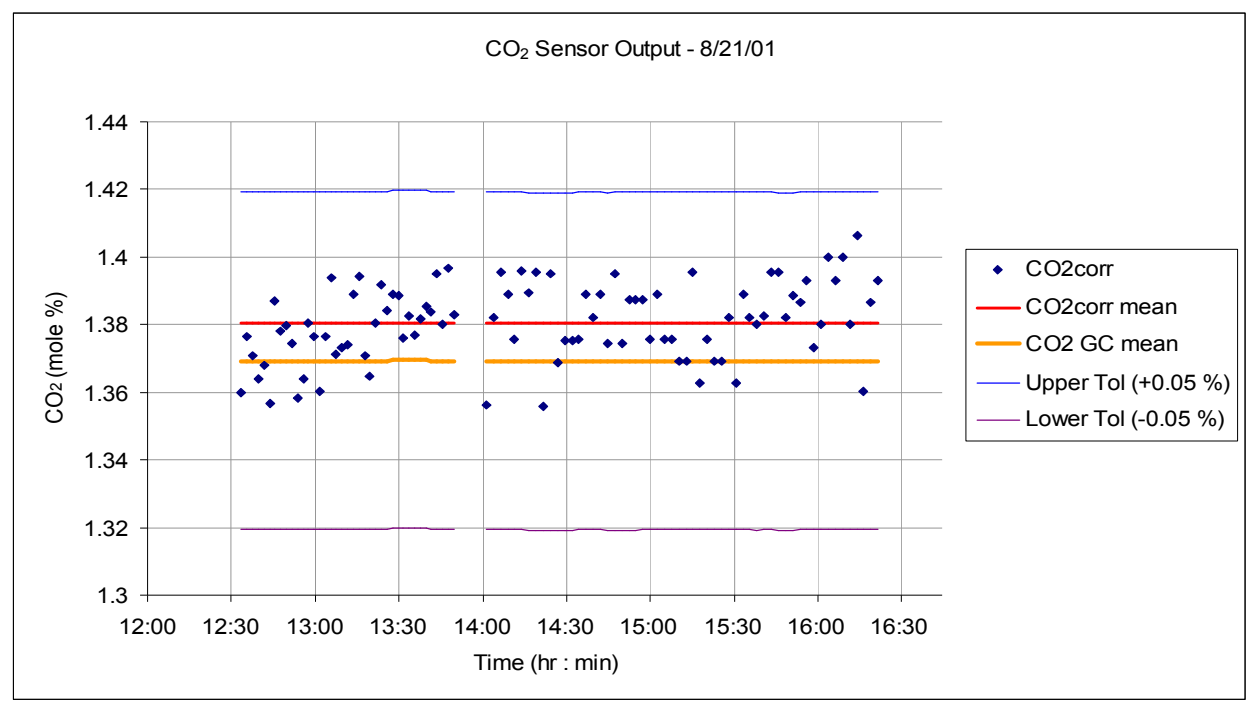

Figure 3.50. MRF testing of $\mathrm{CO}_{2}$ sensor on $8 / 21 / 01$ in HPL.

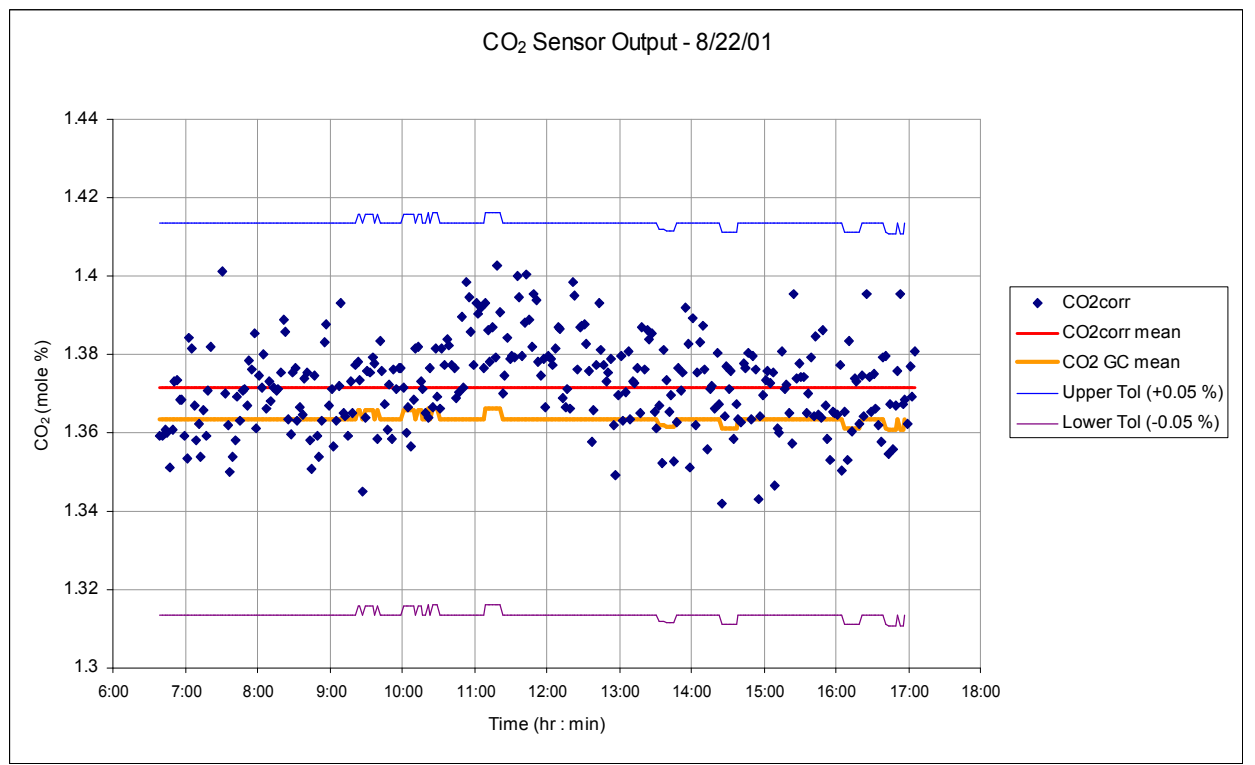

Figure 3.51. MRF testing of $\mathrm{CO}_{2}$ sensor on $8 / 22 / 01$ in HPL. 


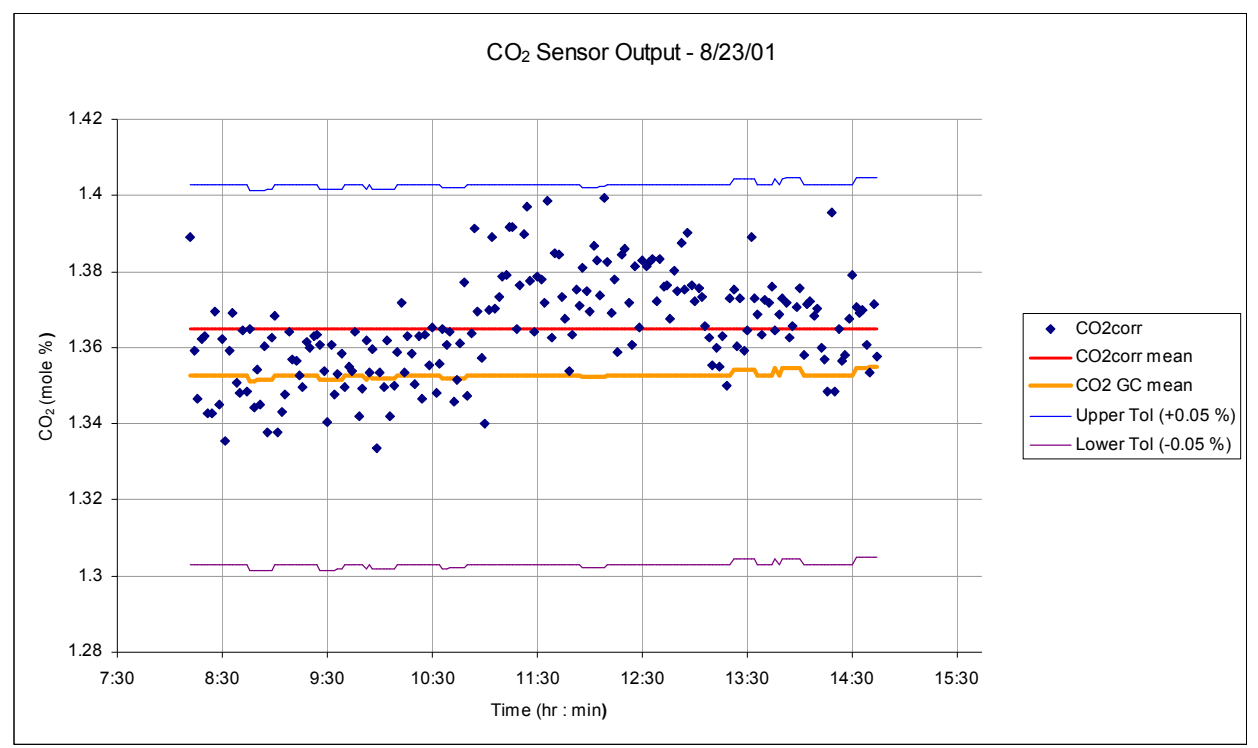

Figure 3.52. MRF testing of $\mathrm{CO}_{2}$ sensor on $8 / 23 / 01$ in HPL.

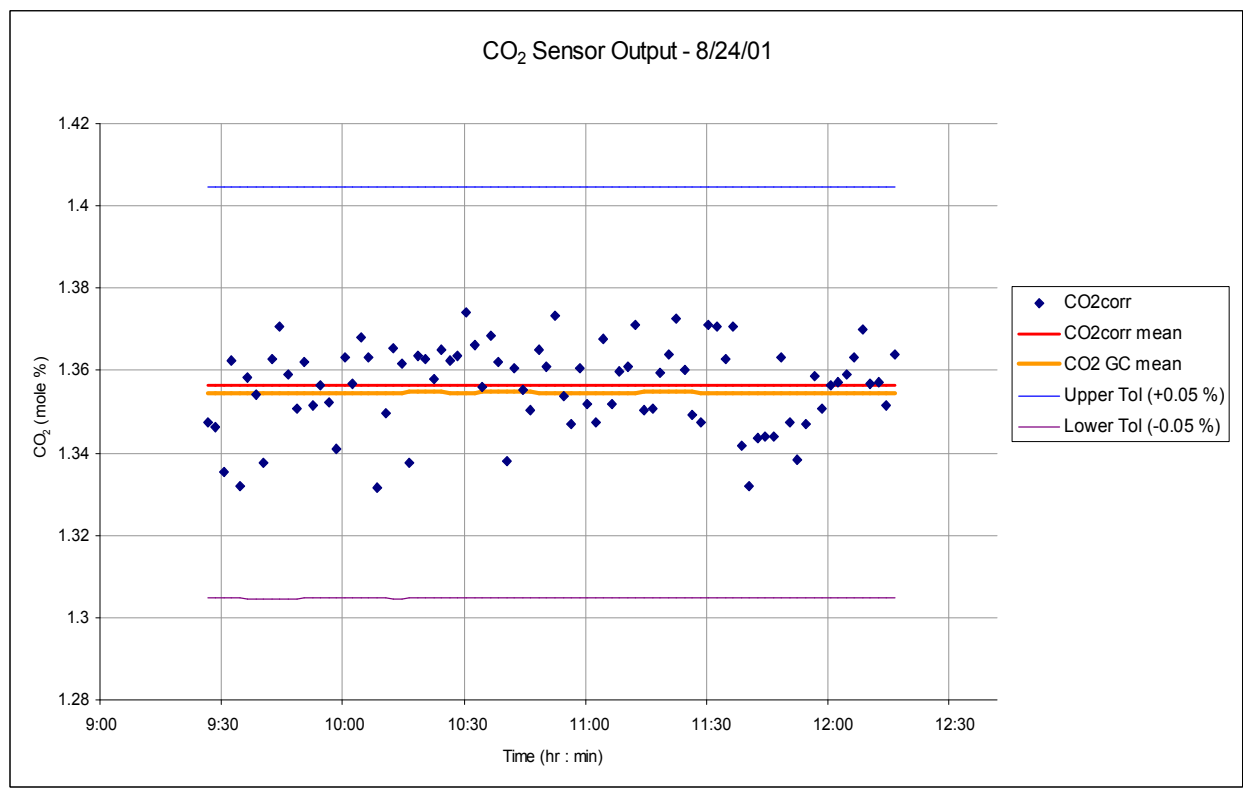

Figure 3.53. MRF testing of $\mathrm{CO}_{2}$ sensor on $8 / 24 / 01$ in $\mathrm{HPL}$. 


\subsubsection{Low-Pressure Loop Tests}

Tests performed in the MRF LPL involved natural gas with $\mathrm{CO}_{2}$ content in the range of $1.135-1.150 \mathrm{~mol} \%$ and natural gas doped with dry $\mathrm{CO}_{2}$ to achieve concentrations of approximately 3, 6, and $9 \mathrm{~mol} \%$. Ambient temperatures in the energy meter module during the various test runs ranged from about $81^{\circ} \mathrm{F}$ to about $97^{\circ} \mathrm{F}$. Data related to $\mathrm{CO}_{2}$ measurements were recorded during approximately 12 hours over a four-day period. The gas temperature, pressure, and flow rate conditions in the loop were varied, and an excess of $\mathrm{CO}_{2}$ was added, resulting in a different overall gas composition on each test day.

Figures 3.54 through 3.60 show the reduced $\mathrm{CO}_{2}$ measurement results for LPL test runs conducted on September 6-11, 2001. The MRF GC $\mathrm{CO}_{2}$ values are shown by the heavy lines and are assumed to be accurate reference values representing the $\mathrm{CO}_{2}$ content in the flow loop gas. The thin lines define the upper and lower tolerance bounds ( $\pm 0.05 \mathrm{~mol} \%$ ref GC value) in which the $\mathrm{CO}_{2}$ sensor measurements must fall in order to yield gas heating values within an accuracy of $\pm 1 \mathrm{Btu} / \mathrm{scf}$. The data points show the temperature- and pressure-corrected $\mathrm{CO}_{2}$ sensor measurements recorded at two-minute intervals during the test runs.

Measurements in the normal loop gas are shown in Figures 3.54 and 3.55. Except for a few anomalous points recorded during the two runs, these measurements were within the tolerance bounds for accurate determination of gas heating value, with the longer test run (approx. $4 \mathrm{hrs)}$ in Figure 3.54 having a scatter of about $\pm 0.015 \mathrm{~mol} \%$. The excursions outside of the upper and lower bounds (extremes of $+0.1 \mathrm{~mol} \%$ and $-0.07 \mathrm{~mol} \%$ ) occurred during the times when adjustments were being made to the $\mathrm{CO}_{2}$ test chamber gas pressure and outlet flow rate and were concluded to be anomalous. Adjustments of this type were not required during the HPL tests previously described. When these wider variations are disregarded, the performance of the $\mathrm{CO}_{2}$ sensor when operating in natural gas with $\mathrm{CO}_{2}$ content in the range of $1.135-1.150 \mathrm{~mol} \%$ in the LPL was comparable with the performance observed in the HPL.

The LPL was doped with dry $\mathrm{CO}_{2}$ on September 7, 2001 to adjust the gas $\mathrm{CO}_{2}$ content to a value near the full-scale operating limit of the $\mathrm{CO}_{2}$ sensor $(10 \mathrm{~mol} \%)$. Figure 3.56 shows the test results obtained during this test run. The adjusted $\mathrm{CO}_{2}$ content stabilized at $9.98 \mathrm{~mol} \%$ and measurements were recorded for approximately 50 minutes. A small amount of natural gas was then introduced to change the loop $\mathrm{CO}_{2}$ concentration to approximately $9.81 \mathrm{~mol} \%$ and, after stabilization, the $\mathrm{CO}_{2}$ sensor measurements were recorded for a period of about 30 minutes. The MRF GC values are also shown in this figure, including the delayed-update GC response to the step change in new gas added. These high-range $\mathrm{CO}_{2}$ measurements exhibited a positive offset of $+7.3 \%$ of reading during the first part of the test and $+6.4 \%$ of reading during the second part of the test. The scatter in the measurements was approximately $\pm 0.06 \mathrm{~mol} \%$ during the first part and, disregarding two anomalous points showing extreme variations of about $\pm 0.1 \mathrm{~mol} \%$, the scatter was within $\pm 0.045 \mathrm{~mol} \%$ during the second part. The offset response and the somewhat wider scatter range in these data were characteristic of a nonlinear response in the $\mathrm{CO}_{2}$ sensor, a condition that could be compensated by a more elaborate sensor linearization and calibration process than presently used.

Figure 3.57 shows the test results obtained with the LPL GC gas $\mathrm{CO}_{2}$ concentration stabilized at $6.12 \mathrm{~mol} \%$. The sensor measurements had a $+6.9 \%$ offset and a scatter of \pm 0.04 $\mathrm{mol} \%$ about the measured mean value. These results at $6 \mathrm{~mol} \%$ followed the same nonlinear trend found in the $9 \mathrm{~mol} \%$ test results. 


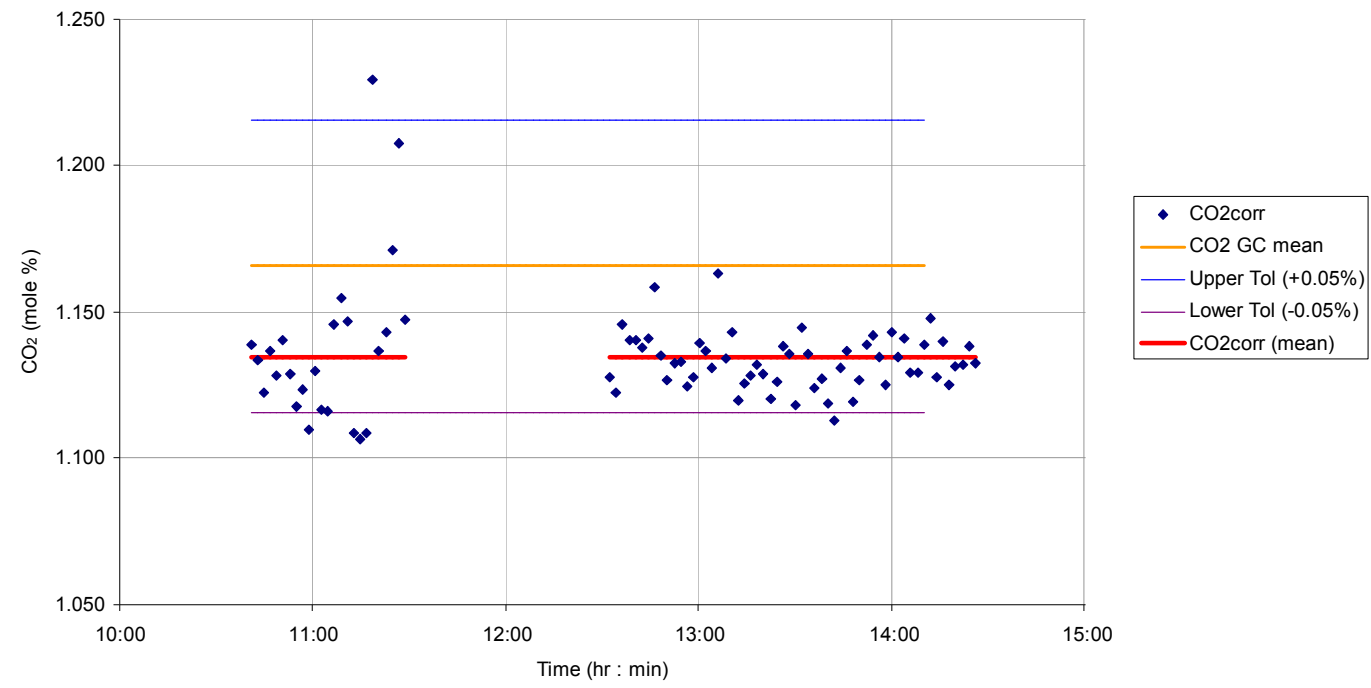

Figure 3.54. MRF LPL testing of $\mathrm{CO}_{2}$ sensor on $9 / 6 / 01\left(\mathrm{CO}_{2}\right.$ av $\left.=1.16 \mathrm{~mol} \%\right)$.

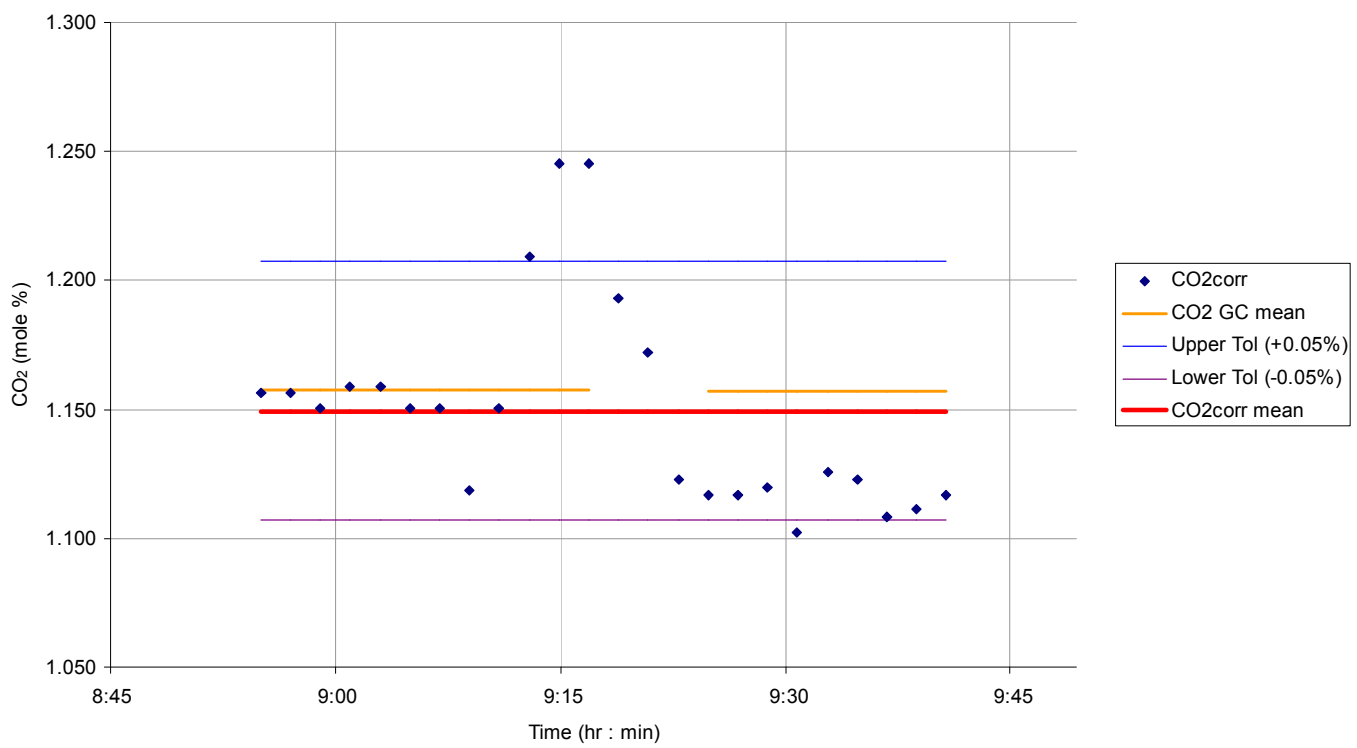

Figure 3.55. MRF LPL testing of $\mathrm{CO}_{2}$ sensor on $9 / 7 / 01\left(\mathrm{CO}_{2} \mathrm{av}=1.15 \mathrm{~mol} \%\right)$. 


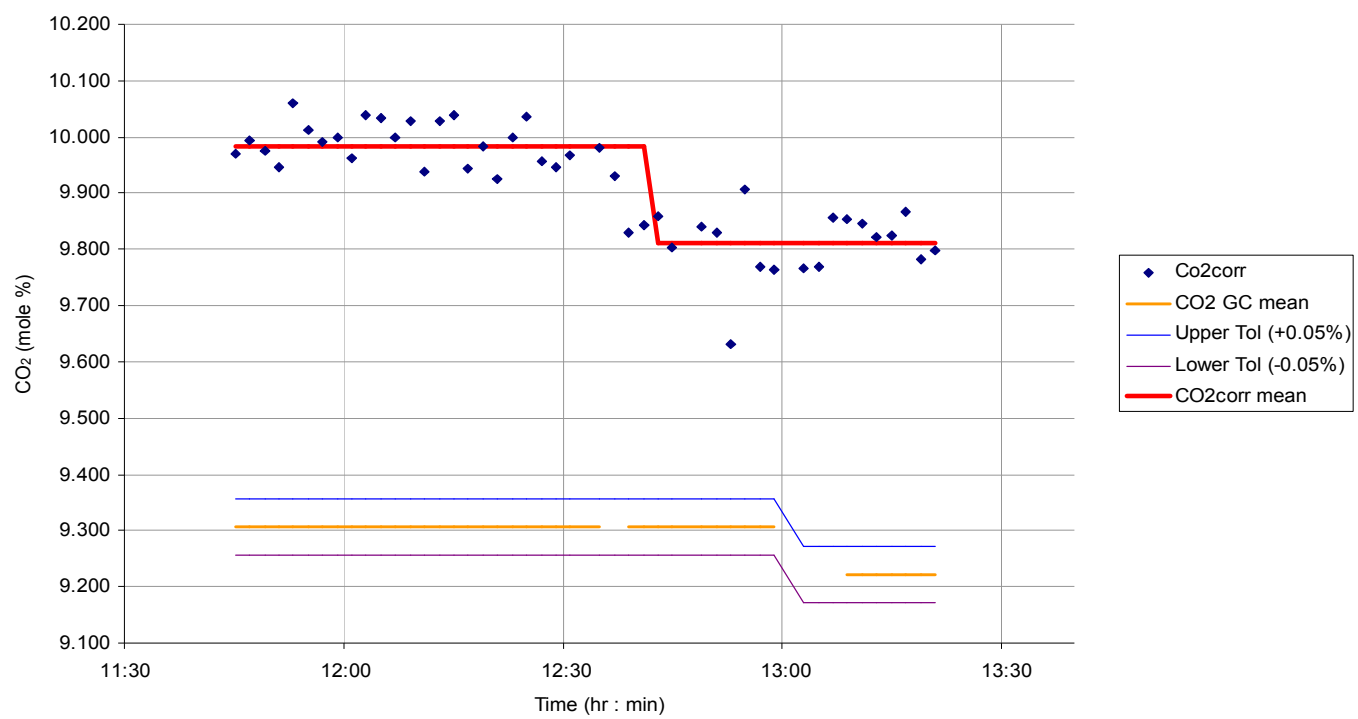

Figure 3.56. MRF LPL testing of $\mathrm{CO}_{2}$ sensor on $9 / 7 / 01\left(\mathrm{CO}_{2}\right.$ av $\left.=9.3 \mathrm{~mol} \%\right)$.

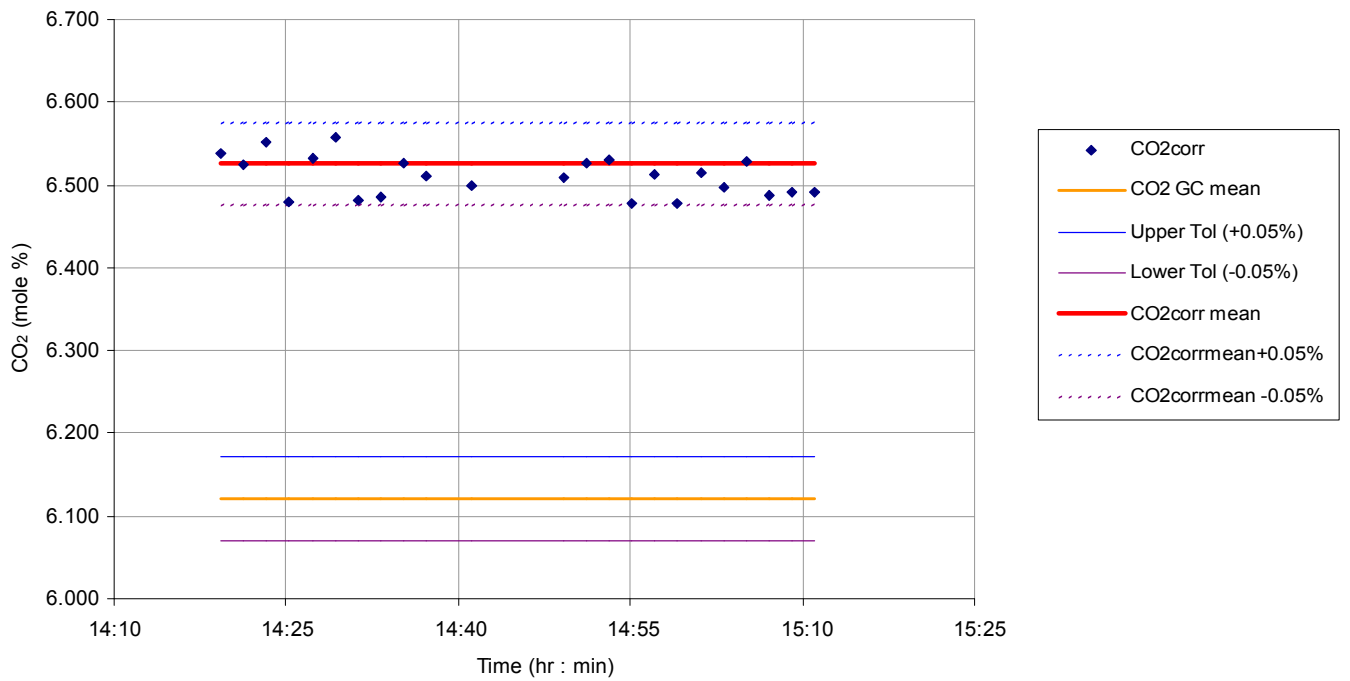

Figure 3.57. MRF LPL testing of $\mathrm{CO}_{2}$ sensor on $9 / 7 / 01\left(\mathrm{CO}_{2} \mathrm{av}=6.12 \mathrm{~mol} \%\right)$. 
Figure 3.58 shows the test results obtained with the LPL GC gas $\mathrm{CO}_{2}$ concentration stabilized first at $3.172 \mathrm{~mol} \%$ for a time period of about 45 minutes, followed by introducing a small amount of new gas to change the concentration to $3.052 \mathrm{~mol} \%$ for a period of two hours and 40 minutes. The sensor measurements showed a $+6.9 \%$ offset and a scatter of $\pm 0.04 \mathrm{~mol} \%$ about the measured mean value during the first interval and an offset of $+6.5 \%$ and a scatter of $\pm 0.035 \mathrm{~mol} \%$ during the first half of the second interval. Anomalous $\mathrm{CO}_{2}$ sensor readings at extremes of $+0.09 \mathrm{~mol} \%$ and $-0.08 \mathrm{~mol} \%$ were present during the latter part of the second interval when flow and pressure adjustments were being made at the energy meter module. These results at $3 \mathrm{~mol} \%$ followed the same nonlinear trend found in the $6 \mathrm{~mol} \%$ and $9 \mathrm{~mol} \%$ test results.

LPL tests were repeated for $\mathrm{CO}_{2}$ concentrations of $6 \mathrm{~mol} \%$ and $9 \mathrm{~mol} \%$ in an effort to obtain measurements having less scatter associated with adjustments to the energy meter gas exit flow rate. Brief test runs at these two concentrations after flow loop conditions stabilized are shown in Figures 3.59 and 3.60. The results indicated a significant reduction in the variations about the mean values for the $6 \mathrm{~mol} \%$ run, but not for the $9 \mathrm{~mol} \% \mathrm{run}$, and confirmed the presence of the nonlinear offsets in the sensor response in both runs. The measurement offsets were $+7.7 \%$ above the $\mathrm{GC}$ value with a maximum scatter range of $\pm 0.06 \mathrm{~mol} \%$ for the $6 \mathrm{~mol} \%$ test run and $+10.6 \%$ for the $9 \mathrm{~mol} \%$ measurements with two isolated but extreme variations of $+0.45 \mathrm{~mol} \%$ in the $9 \mathrm{~mol} \%$ test run.

In general, for the ambient operating conditions that existed during the LPL tests in which natural gas with $\mathrm{CO}_{2}$ content in the range of $1.135-1.150 \mathrm{~mol} \%$ was used, the technical performance of the Vaisala GMP221B $\mathrm{CO}_{2}$ sensor performed with sufficient accuracy and stability for use in the energy meter measurement application. Although some extreme variations were observed in these tests, the scatter in the measurements is concluded to be an artifact associated with the sparse data logging process (one sample point every two minutes) when adjustments of pressure and flow rate were being made in the energy meter module. The LPL tests conducted using natural gas doped to higher $\mathrm{CO}_{2}$ concentrations provided insights into the degree of non-linearity in the sensor response and indicated the presence of larger scatter in the measurements at higher concentrations. These anomalous effects could be eliminated in an improved prototype energy meter module by using a preset flow control valve designed to operate at the 100 psia-to-15 psia pressure reduction between the speed-of-sound test chamber and the $\mathrm{CO}_{2}$ test chamber. The nonlinear sensor response is a more fundamental concern that will require further evaluation. Although the degree of non-linearity exhibited by the Vaisala GMP221B sensor is of minimum consequence at low $\mathrm{CO}_{2}$ concentrations (1-2 mol\%), this source of error must be corrected by a linearization process that encompasses the full environmental range of operation expected for the energy meter application. A first approach to this correction should be a linear temperature scaling combined with a polynomial in pressure in place of the linear pressure scaling factor. 


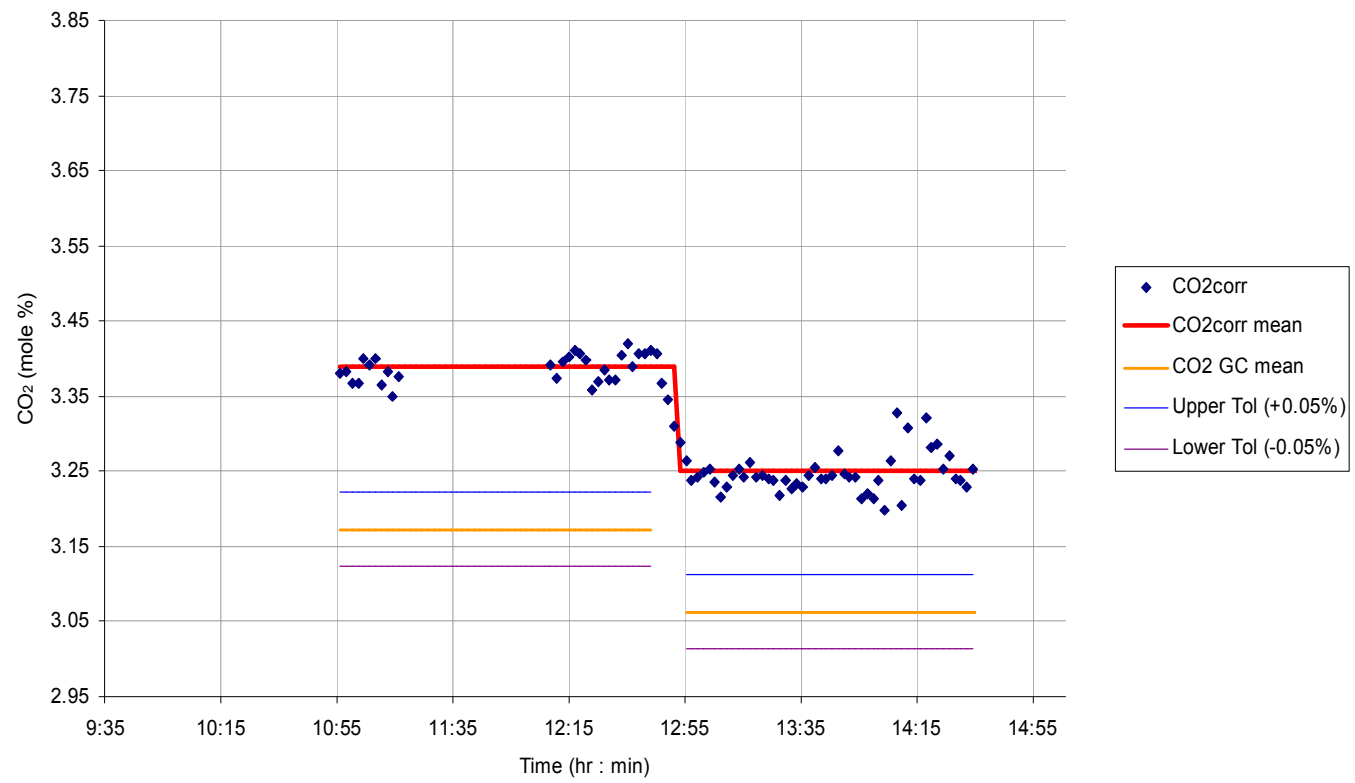

Figure 3.58. MRF LPL testing of $\mathrm{CO}_{2}$ sensor on $9 / 10 / 01\left(\mathrm{CO}_{2} \mathrm{av}=3.15 \mathrm{~mol} \%\right)$.

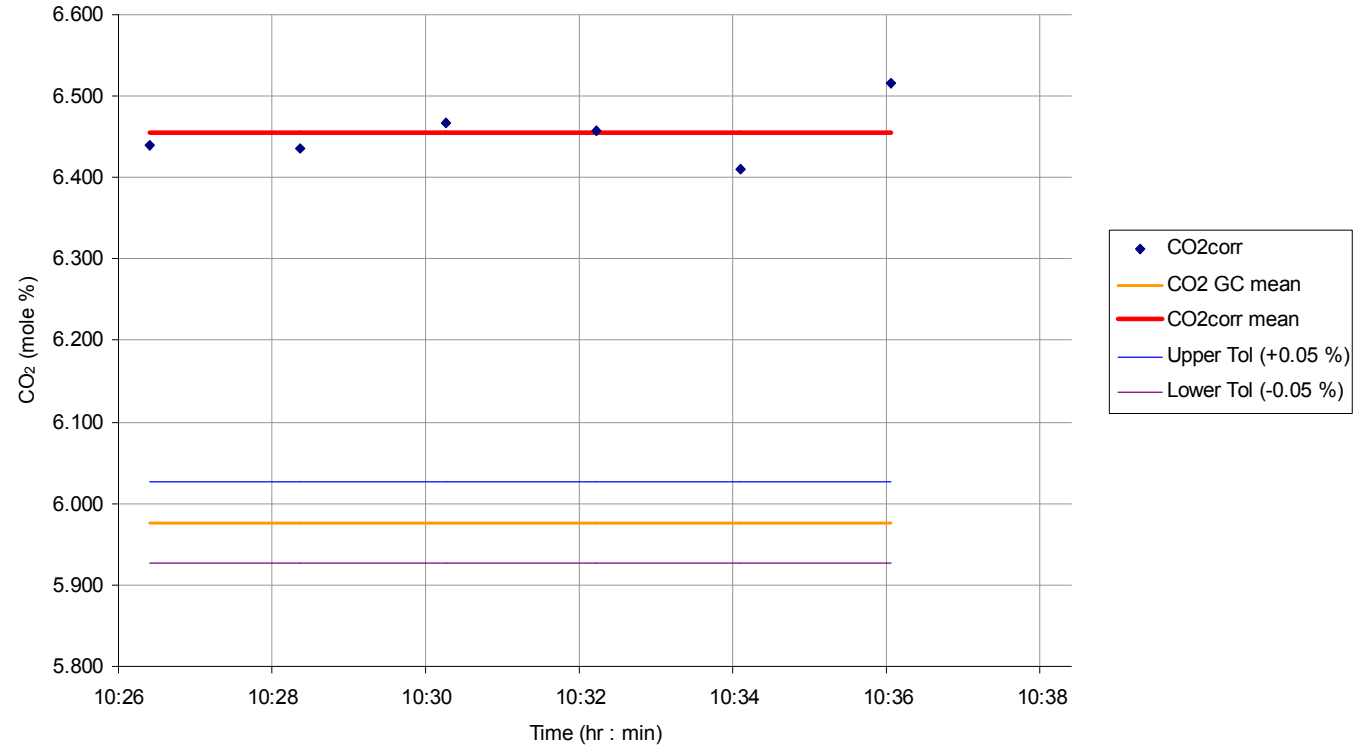

Figure 3.59. MRF LPL testing of $\mathrm{CO}_{2}$ sensor on $9 / 10 / 01\left(\mathrm{CO}_{2} \mathrm{av}=5.98 \mathrm{~mol} \%\right)$. 


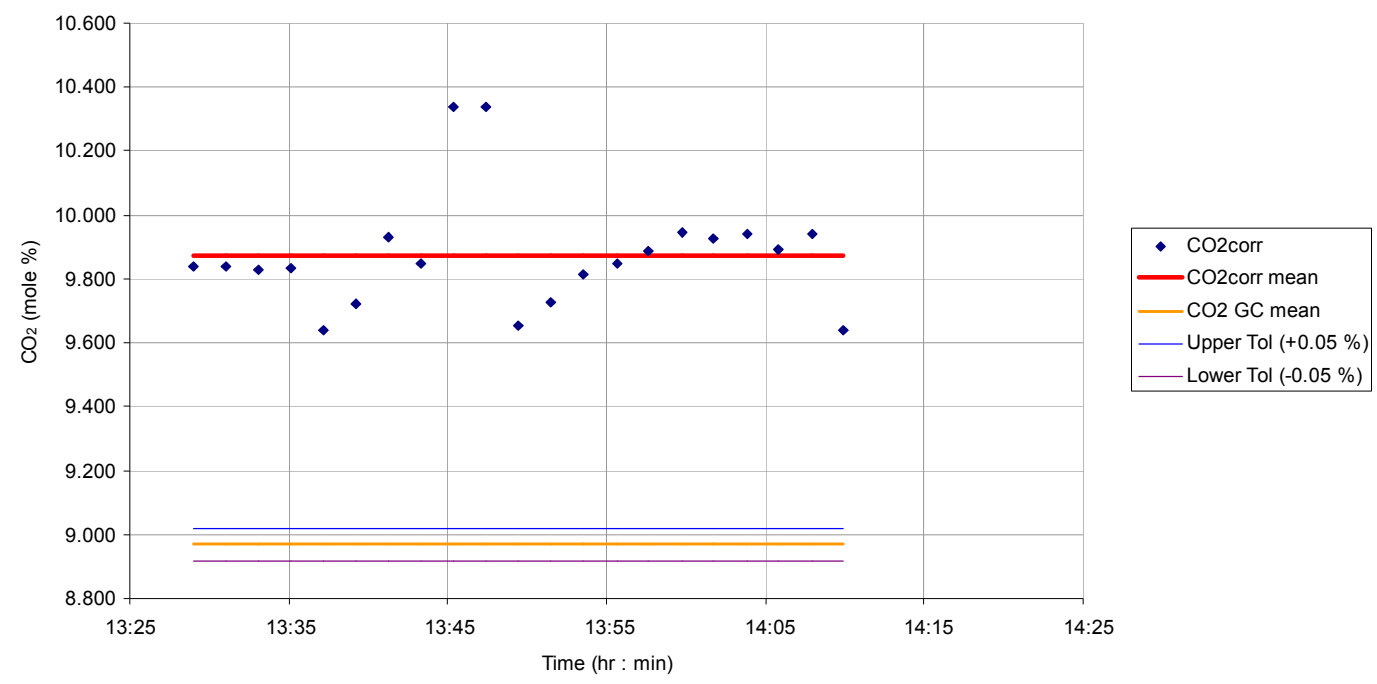

Figure 3.60. MRF LPL testing of $\mathrm{CO}_{2}$ sensor on $9 / 11 / 01\left(\mathrm{CO}_{2 \mathrm{av}}=8.98 \mathrm{~mol} \%\right)$.

\subsection{Evaluation of Inferential Techniques for Sensing $\mathbf{N}_{\mathbf{2}}$ Concentration}

During the 1999-2000 project year, a method to infer nitrogen concentration from the measurement of the speed of sound in natural gas at two different pressures was proposed. This method was found to be impractical because it would require a measurement precision believed to be unattainable in the field. For the 2000-2001 project year, several other methods for inferring nitrogen concentration were evaluated. One approach was based on a GRI patent and required measurement of the specific heat of the gas at constant volume. Three other methods were investigated that have been tested at Southwest Research Institute and elsewhere for their ability to characterize natural gas and hydrocarbon fuels. The characteristics of these four methods are summarized in Table 3.7. A fifth approach was conceived during these studies after a review of the properties of natural gas components and the speed-of-sound measurements in the current module design. The five methods are described and evaluated in this section.

\subsubsection{GRI Isochoric Approach}

The first method considered for inferring nitrogen content is based upon GRI patent 5,932,793 [Dayton et al. (1999)]. This method involves measuring the speed of sound of the gas in a constant-volume vessel at different temperatures and pressures, then using the measurements to determine the specific heats of the gas at constant pressure and constant volume.

To apply this approach to the energy meter, the iterative method for computing $c_{p}$ and $c_{v}$ in the patent would be included in the algorithm. The specific heats would replace nitrogen content as independent variables, and a new correlation for $\mathrm{N}_{2}$ content as a function of sound speed, $\mathrm{CO}_{2}$ content, temperature, pressure, $\mathrm{c}_{\mathrm{p}}$, and $\mathrm{c}_{\mathrm{v}}$ would be applied. Several design changes 
Table 3.7. Comparison of five potential methods for sensing nitrogen content in natural gas.

\begin{tabular}{|c|c|c|c|c|c|}
\hline Method & $\begin{array}{l}\text { Isochoric/isobaric/ } \\
\text { isothermal } \\
\text { measurements } \\
\text { (Savidge/GRI) }\end{array}$ & $\begin{array}{l}\text { "Microbrick" } \\
\text { sensor (Bonné, } \\
\text { Honeywell) }\end{array}$ & $\begin{array}{l}\text { Laminar flow } \\
\text { sensor (Bonné, } \\
\text { Honeywell) }\end{array}$ & $\begin{array}{l}\text { Hydrogen transient } \\
\text { nuclear magnetic } \\
\text { resonance } \\
\text { (Callahan, SwRI) }\end{array}$ & $\begin{array}{l}\text { Near-infrared } \\
\text { spectrometer } \\
\text { (Westbrook, } \\
\text { SwRI) }\end{array}$ \\
\hline Principle & $\begin{array}{l}\text { Changes in gas } \\
\text { properties along } \\
\text { curves of constant } \\
\rho / \mathrm{P} / \mathrm{T} \text { are found } \\
\text { by changing sample } \\
T \text { and } \mathrm{P} \text {. }\end{array}$ & $\begin{array}{l}\text { Pairs of acoustic } \\
\text { actuators create } \\
\text { pulsations in gas } \\
\text { chamber; voltage } \\
\text { response of "micro- } \\
\text { brick" anemometers } \\
\text { used to infer flow } \\
\text { rate and gas } \\
\text { properties. }\end{array}$ & $\begin{array}{l}\text { Pairs of acoustic } \\
\text { actuators create } \\
\text { pulsations in a } \\
\text { laminar flow ele- } \\
\text { ment; pressure } \\
\text { drop across LFE } \\
\text { and Poiseuille's law } \\
\text { used to infer } \\
\text { viscosity. }\end{array}$ & $\begin{array}{l}\text { Amplitude and time } \\
\text { constants of } \\
\text { response to magnetic } \\
\text { pulses yield total } \\
\text { hydrogen content and } \\
\text { HC composition. }\end{array}$ & $\begin{array}{l}\text { Absorption bands } \\
\text { of HCs in near-IR } \\
\text { region, overtones } \\
\text { of known bands in } \\
\text { mid-IR region, are } \\
\text { used to measure } \\
\text { HC ratios. }\end{array}$ \\
\hline $\begin{array}{l}\text { Quantities } \\
\text { measured } \\
\text { directly }\end{array}$ & Sound speed, P, T & $\begin{array}{l}\text { Voltage output from } \\
\text { anemometers }\end{array}$ & $\begin{array}{l}\text { Pressure difference } \\
\text { across laminar flow } \\
\text { element }\end{array}$ & $\begin{array}{l}\text { NMR signal over } \\
\text { time; amplitude, time } \\
\text { constants } T_{1} \text { and } T_{2}\end{array}$ & $\begin{array}{l}\text { IR absorption } \\
\text { spectra }\end{array}$ \\
\hline $\begin{array}{l}\text { Quantities } \\
\text { derived }\end{array}$ & $\begin{array}{l}\mathrm{Z}, \rho, \mathrm{c}_{\mathrm{v}}, \mathrm{c}_{\mathrm{p}} \text { and } \\
\text { derivatives }\end{array}$ & $\begin{array}{l}\mathrm{Q}, \mathrm{c}_{\mathrm{p}}, \mathrm{k}, \\
\text { composition } \\
\text { correction factor } \mathrm{C}_{\mathrm{v}} \text {; } \\
\text { potentially } \mathrm{\gamma}, \mathrm{H}_{\mathrm{v}}\end{array}$ & $\begin{array}{l}\text { Gas dynamic } \\
\text { viscosity, } \mu\end{array}$ & $\begin{array}{l}\text { Partial pressures } \\
\text { (mole fractions) of } \\
\text { hydrogen-bearing } \\
\text { gases; total gas } \\
\text { pressure; } \mathrm{H}_{v} \\
\end{array}$ & $\begin{array}{l}\mathrm{H}_{\mathrm{m}} \text {, Wobbe index, } \\
\text { hydrogen-to- } \\
\text { carbon ratio }\end{array}$ \\
\hline $\begin{array}{l}\text { How method } \\
\text { could be } \\
\text { included in } \\
\text { energy meter } \\
\text { approach }\end{array}$ & $\begin{array}{l}\text { Correlate specific } \\
\text { heats to } \mathrm{N}_{2} \text { content } \\
\text { and other } \\
\text { quantities. } \\
\rho \text { would come from } \\
\text { algorithm in GRI } \\
\text { patent instead of } \\
\text { inferential } \\
\text { correlation. }\end{array}$ & $\begin{array}{l}\text { Correlate derived } \\
\text { properties to } \mathrm{N}_{2} \\
\text { content and other } \\
\text { final quantities }\end{array}$ & $\begin{array}{l}\text { Correlate viscosity } \\
\text { to } \mathrm{N}_{2} \text { content and } \\
\text { other quantities. }\end{array}$ & $\begin{array}{l}\text { Find mole fractions of } \\
\text { hydrogen-bearing } \\
\text { gases; remaining } \\
\text { fractions can be } \\
\text { attributed to inert } \\
\text { gases. Subtract } \mathrm{CO}_{2} \\
\text { from the inert mole } \\
\text { fraction to get } \mathrm{N}_{2} \text {. }\end{array}$ & $\begin{array}{l}\text { 1) Find relative } \\
\text { mole fractions of } \\
\mathrm{HCs} \text {, but not clear } \\
\text { how to find total } \mathrm{H}- \\
\text { bearing mole frac- } \\
\text { tion from NIR data. } \\
\text { 2) Correlate } \\
\text { Wobbe index to } \mathrm{N}_{2} \\
\text { content and other } \\
\text { quantities? }\end{array}$ \\
\hline $\begin{array}{l}\text { Some } \\
\text { required } \\
\text { changes to } \\
\text { module if } \\
\text { method } \\
\text { included }\end{array}$ & $\begin{array}{l}\text { Temperature } \\
\text { control, control } \\
\text { valve, hydraulic } \\
\text { manifold. }\end{array}$ & $\begin{array}{l}\text { Microbrick sensors } \\
\text { added to module } \\
\text { and spool piece in } \\
\text { main flow; heater } \\
\text { may be needed. }\end{array}$ & $\begin{array}{l}\text { Laminar flow } \\
\text { sensors added to } \\
\text { module, in line with } \\
\text { existing sensors. }\end{array}$ & $\begin{array}{l}\text { RF coil and per- } \\
\text { manent magnets, } \\
\text { nonmetallic pipe for } \\
\text { sample. }\end{array}$ & $\begin{array}{l}\text { NIR probe and } \\
\text { fiber optics, spec- } \\
\text { trometer, helium } \\
\text { supply and means } \\
\text { to purge test cell. }\end{array}$ \\
\hline $\begin{array}{l}\text { Estimated } \\
\text { cost of new } \\
\text { hardware }\end{array}$ & No estimate. & $\begin{array}{l}\text { Inexpensive } \\
\text { microsensor when } \\
\text { produced in } \\
\text { quantity }\end{array}$ & $\begin{array}{l}\text { Inexpensive } \\
\text { microsensor when } \\
\text { produced in } \\
\text { quantity }\end{array}$ & Excessive & $\begin{array}{l}\text { Spectrometer } \\
\text { costly }\end{array}$ \\
\hline $\begin{array}{l}\text { Test } \\
\text { conditions }\end{array}$ & No applied tests. & $\begin{array}{l}\text { Rated at }-40^{\circ} \mathrm{C} \\
\text { to } 150^{\circ} \mathrm{C}\end{array}$ & $\begin{array}{l}\text { Evaluated from } 1 \text { to } \\
7 \text { bar, } 0 \text { to } 40^{\circ} \mathrm{C}\end{array}$ & $\begin{array}{l}\text { } 50 \text { to } 1000 \text { psig, } \\
0.5 " \text { ID sample pipe }\end{array}$ & 30 psig, $23^{\circ} \mathrm{C}$ \\
\hline Accuracy & Unknown. & $\mathrm{c}_{\mathrm{p}}, \mathrm{k}$ to $\pm 1 \%$ & $\mu$ to $\pm 0.3 \%$ & $\begin{array}{l}\mathrm{P} \text { to } 10 \% ; \mathrm{H}_{\mathrm{v}} \text { to } 1.5 \% \\
\text { if } \mathrm{P} \text { measured } \\
\text { independently }\end{array}$ & $\begin{array}{l}\mathrm{H}_{\mathrm{m}} \text { to } 2 \%, \mathrm{H} / \mathrm{C} \\
\text { ratio to } 3.5 \% \\
\text { (sensitive to pres- } \\
\text { sure and flow rate) }\end{array}$ \\
\hline $\begin{array}{l}\text { Time for one } \\
\text { set of } \\
\text { measure- } \\
\text { ments }\end{array}$ & $\begin{array}{l}\text { Unknown, but could } \\
\text { be long; } \\
\text { measurements } \\
\text { along several } \\
\text { isochores required. }\end{array}$ & Unknown & $\begin{array}{l}\text { Less than one } \\
\text { minute }\end{array}$ & $\begin{array}{l}10-25 \text { acquisitions } \\
\text { in } 1 \text { minute }\end{array}$ & $100 \mathrm{sec}$. \\
\hline Comments & $\begin{array}{l}\text { Several errors in } \\
\text { formulas found in } \\
\text { the patent }\end{array}$ & $\begin{array}{l}\text { System must be } \\
\text { calibrated to } C_{v} \\
\text { values of known } \\
\text { gases. Previous } \\
\text { work at SwRI } \\
\text { patented by } \\
\text { customer; sensors } \\
\text { in production. }\end{array}$ & $\begin{array}{l}\text { Sensor must be } \\
\text { calibrated to } \\
\text { viscosity values of } \\
\text { known gases. }\end{array}$ & $\begin{array}{l}\text { Need NMR data at } \\
\text { higher pressures, for } \\
\text { heavier } \mathrm{HCs} \text { and } \\
\text { mixtures with inert } \\
\text { gases. } \mathrm{H}_{2} \mathrm{O} \text { and } \mathrm{H}_{2} \mathrm{~S} \\
\text { might require special } \\
\text { attention. }\end{array}$ & $\begin{array}{l}\text { Multivariate } \\
\text { calibration required } \\
\text { for known gases, } \\
\text { since peaks in NIR } \\
\text { tend to overlap. } \\
\text { Need more study } \\
\text { of T, P effects, and } \\
\text { inert gas effects. }\end{array}$ \\
\hline
\end{tabular}


to the existing module would also be required, including a method for controlled heating of the gas, a control valve to regulate pressure, and a hydraulic manifold. Measurements along several constant-volume curves would be required to obtain specific heats, potentially extending the time needed to obtain the gas heating value. While the approach in the patent is believed to be sound, no known tests of the method have been conducted, and the feasibility of its use in the module is not as promising as some of the other methods suggested below.

\subsubsection{Honeywell Natural Gas Sensor}

The second approach evaluated for nitrogen measurement involves sensors developed at Honeywell Laboratories for measuring natural gas properties [Bonné and Kubisiak (2001)]. This approach uses actuators, or "speakers," to induce pressure pulsations in the gas, but can incorporate two different techniques to measure gas properties once the pulsations have been created.

The first technique devised for use with the actuators employs a commercial thermal anemometer, modified for use in flammable gases, particulate flows, and high flow rates up to $200 \mathrm{~m} / \mathrm{s}$. A pair of these ruggedized anemometers, or "microbricks," is used to simultaneously measure gas properties in a main gas line and in a separate normalizing chamber that draws gas from the main line. An actuator sends acoustic pulses at the speed of sound through the sample in the normalizing chamber, and the difference in response of the gas to the flows at the two different locations is measured by the microsensors. This approach can be used to determine pipeline flow rates, but also has the potential to measure gas properties, including specific heat at constant pressure $\left(\mathrm{c}_{\mathrm{p}}\right)$, thermal conductivity $(\mathrm{k})$, and a volumetric composition correction factor $\mathrm{C}_{\mathrm{v}}$ devised by Bonné and Kubisiak [2001] to determine compositions of binary mixtures. The microbrick approach was recently used at Southwest Research Institute to test the performance of natural-gas-fueled vehicles. When the microsensors were used primarily to determine oxygen demand of the vehicle engines, values of $c_{p}$ and $k$ were also measurable with $1 \%$ accuracy.

A second technique was developed and tested at Honeywell that uses time-varying actuators to create a flow-induced amplitude modulated pressure drop across a laminar flow restrictor, and a direct measurement of pressure drop across the restrictor to infer the gas absolute viscosity $(\mu)$. Unlike the microbrick technique, all measurements occur in a single chamber that can be used to draw samples from a gas line. Because of the laminar flow device, however, the gas samples must be drawn at low flow rates and filtered for particulates. In published tests [Bonné and Kubisiak (2001)], a calibrated sensor was able to measure gas viscosity with a repeatability of $\pm 0.3 \%$.

To apply this method in the energy meter, microsensors would be placed in the main flow and in a separate normalizing chamber included in the stand-alone module. Flow straighteners would be required on either side of the sensor in the main flow, and depending on the temperature of the gas, a heater element may be required to condition the normalizing sample. Such an approach would be more appropriate for an energy meter produced as an in-line spool piece. The laminar flow approach for viscosity would be simpler to implement with a standalone module, since the entire self-contained sensor could use the same sample stream as the speed-of-sound and $\mathrm{CO}_{2}$ sensors. In either case, low-cost actuators would be sufficient to produce pulses for the sensors, rather than the transducer now used for speed-of-sound measurements. 
With either technique, the gas property or properties measured by the sensors would become independent variables in the algorithm, and would have to be accurately correlated to nitrogen content. Test correlations were performed to determine the gas property measured by the microsensor that would best predict nitrogen content and density at STP (hence heating value). Correlations of $\rho_{\text {STP }}$ and nitrogen concentration to speed of sound, $\mathrm{CO}_{2}$ content, and each of the candidate properties were computed using data from the GERG database [Starling et al. (1991)] and the GRI Gas Resource Database [Gas Research Institute (1998)] for gases with 6 mol\% $\mathrm{N}_{2}$ or less. The best correlations were related to the specific heat quantities, $\mathrm{c}_{\mathrm{p}}$ and $\gamma$, followed by viscosity and thermal conductivity. Correlations with $\mathrm{c}_{\mathrm{p}}$ and $\gamma$ predicted density at STP to within a very acceptable accuracy of $\pm 0.05 \%$, but could only predict $\mathrm{N}_{2}$ concentration to within $\pm 0.25 \mathrm{~mol} \%$ ( $\pm 0.05 \mathrm{~mol} \%$ required $)$. Correlations with viscosity predicted $\rho_{\text {STP }}$ to within $\pm 0.08 \%$, also an acceptable accuracy, but could only predict $\mathrm{N}_{2}$ concentration to within \pm 0.31 $\mathrm{mol} \%$.

Correlations were also performed on data from the same two databases with $\mathrm{CO}_{2}$ and $\mathrm{N}_{2}$ up to $20 \mathrm{~mol} \%$, using $\mathrm{c}_{\mathrm{p}}$ as the measured independent variable. Molecular weight and $\rho_{\mathrm{STP}}$ correlated to specific heat and the other inputs to within $\pm 0.02 \%$, but again, nitrogen content did not correlate to $\mathrm{c}_{\mathrm{p}}$ as accurately. To solve this problem, it may be possible to rearrange the correlation of molecular weight to temperature, pressure, speed of sound, $\mathrm{CO}_{2}$, and $\mathrm{N}_{2}$ in place of the current algorithm, to make nitrogen content a function of molecular weight. The specific heat measured by the microbrick could be used to find molecular weight, as discussed above, and then a reverse correlation could be applied using molecular weight to find nitrogen concentration.

The Honeywell sensors could be used to accurately measure the properties needed for heating values and energy flow rates, but their ability to accurately infer nitrogen content is not yet certain. Of the measurement methods described in this section that have already been tested, however, these techniques appear to require the least development to be successfully incorporated into the energy meter.

\subsubsection{Nuclear Magnetic Resonance}

A method using nuclear magnetic resonance (NMR) for on-line natural gas measurements, investigated at SwRI [Nicholls and King (1989); King and Nicholls (1990)] and elsewhere, was also reviewed for its potential use in the energy meter package. In NMR, a varying high-strength magnetic field is applied to a gas sample and the amplitude and timing of resonant $\mathrm{RF}$ responses from hydrogen atoms in the gas are measured. The response amplitudes and relaxation times can be used to determine mole fractions of all hydrogen-bearing gases in the sample $\left(\mathrm{H}_{2}, \mathrm{CH}_{4}, \mathrm{C}_{2} \mathrm{H}_{6} \ldots\right)$ as well as the total gas pressure. If the hydrogen and hydrocarbon mole fractions in a natural gas sample could be found by NMR with sufficient accuracy, the $\mathrm{CO}_{2}$ content could also be measured directly with the existing sensor, and the balance of the gas could be assigned to nitrogen, assuming that no other non-hydrogen-bearing diluents are present in the gas.

Laboratory tests at SwRI [King et al. (1988)] demonstrated that NMR has the potential to measure the density and hydrocarbon content of a natural gas stream. However, further tests with the SwRI approach would be needed to extend the method to gases of interest for the energy meter. Testing would be required at high pressures, with samples containing $\mathrm{CO}_{2}$ and $\mathrm{N}_{2}$, and with gases containing heavy hydrocarbons. Supplemental information [De Los Santos (2001)] 
was also reported indicating that high pressures would pose a problem to data acquisition. The NMR system used in the 1988-1990 research was able to measure properties of natural gas flowing through a 0.5 -in. diameter pipe at pressures from 50 to $1000 \mathrm{psig}$, similar to pressures in the transmission industry. Because the time required for the hydrogen nuclei to polarize and "relax" from the magnetic field excitation is proportional to pressure, however, higher pressures require longer data acquisition times. At 1000 psia, a pressure that would likely be encountered by the energy meter, relaxation cycle times of 50 seconds can be expected. At 1500 psia, a single measurement cycle would require about one minute. Signal processing to obtain high signal-tonoise ratios would also increase the time required for accurate measurements. Shorter acquisition times than these would be desirable.

Information on the basic sensing methodology and the cost of including an NMR sensor in the module was obtained as a further aspect of its practicability. Since the sensitivity of NMR to nitrogen itself is three orders of magnitude lower than the sensitivity to hydrogen, the hydrocarbon- $\mathrm{CO}_{2}-\mathrm{N}_{2}$ "balance" method described at the beginning of this subsection would be used. Broadline NMR instruments are capable of distinguishing hydrocarbons up through $\mathrm{C}_{5}$, which would require a "lumped" analysis of the heavier hydrocarbons. A preliminary uncertainty analysis shows that to obtain the nitrogen content and heating value of a gas sample to within $\pm 0.5 \%$, the total uncertainty in hydrocarbons $\mathrm{CH}_{4}$ through $\mathrm{C}_{5} \mathrm{H}_{12}$ found by $\mathrm{NMR}$ must be $\pm 0.3 \%$. By comparison, the 1988 SwRI system was able to determine heating value to $\pm 1.5 \%$ (15 Btu/scf for a $1000 \mathrm{Btu} / \mathrm{scf}$ natural gas).

The cost of including NMR in the energy meter system is prohibitive. If the NMR approach were used, an RF coil, permanent magnets, and non-metallic pipe would be required, requiring major modifications to the module. A basic broadline NMR instrument with a line diameter of 0.37 in., a 0.4 Tesla permanent magnet, and the ability to carry flows at 1500 psia would cost at least $\$ 10,000$ in production quantities [De Los Santos (2001)]; an amount greater than the target price of the energy meter module. A high-resolution NMR system capable of fuel gas analysis and spectroscopy through $\mathrm{C}_{6}$ would be expected to cost $\$ 75,000$. Based on this information, it was decided not to pursue NMR as a method for nitrogen determination for the energy meter application.

\subsubsection{Infrared Spectrometry}

A method for characterizing hydrocarbon fuels involving infrared spectrometry, also developed at SwRI [Westbrook (1993, 1994)], was reviewed for possible use in the energy meter. In this approach, the absorption spectrum of a gas sample in the near-infrared and midinfrared regions is measured and correlated to the hydrogen-to-carbon ratio of the gas. As originally proposed, the method would correlate the absorption spectrum to the Wobbe index and the mass-based heating value of diluent-free natural gas.

This method can also determine the mole fractions of different hydrocarbon gases relative to the most common hydrocarbons (e.g., methane). This information would be useful for the energy meter if the relative mole fractions of non-hydrocarbon diluents could also be obtained in some way. A practical alternative might be to try correlating the Wobbe index to nitrogen content and to gas density, making the index itself the fifth input to the algorithm. However, extensive changes to the module would be required for infrared spectrometry, including an infrared probe, fiber optics, a test cell, a purge mechanism for the test cell, and a spectrometer (or a less expensive method of determining the infrared absorption spectrum). 
The system described by Westbrook was able to obtain a single measurement of an infrared spectrum in 100 seconds, which is much longer than the current measurement cycle in the module. Tests of the method on a natural gas sample at approximately two atmospheres pressure demonstrated that the hydrogen-to-carbon ratio could be determined only to within $3.5 \%$, and this accuracy varied with the pressure and flow rate of the gas sample. Peaks in the near-infrared spectrum tend to overlap for certain gas components, causing uncertainty in the $\mathrm{H} / \mathrm{C}$ ratio, but this problem could be reduced with a multivariate calibration on known gas samples. In summary, more study of the effects of temperature, pressure, and diluent gases is needed before this method could be accurately applied to the energy meter.

\subsubsection{Acoustic Viscosity and Nitrogen Measurements}

In the search for other useful technologies, data on the constituents of natural gas were reviewed to find a measurable property of nitrogen that is distinct from other components. Properties at $298 \mathrm{~K}$ and $1 \mathrm{~atm}$ were compared for pure hydrocarbon gases, nitrogen, carbon dioxide, and other diluents less common in natural gas. Several of the properties reviewed were studied in connection with the microsensor method, but other measurable properties such as thermal diffusivity, the Joule-Thomson coefficient, the Prandtl number, and the dielectric constant were also compared. Three properties were identified that differ significantly between hydrocarbons and nitrogen: the specific heats $c_{p}$ and $c_{v}$, and the absolute viscosity $\mu$. For typical natural gas mixtures containing carbon dioxide, nitrogen, and descending concentrations of hydrocarbons, nitrogen has the highest absolute viscosity, and a constant pressure specific heat that is a factor of two or more lower than the hydrocarbon components. The "microbrick" sensor described earlier has the capability to measure $c_{p}$ and $\mu$, as discussed earlier, but its installation would require some changes in the existing module design.

A measurement method related to viscosity recently identified is one that would use the existing speed-of-sound chamber and require minimal changes to the module design. Viscous friction and molecular vibration modes govern the behavior of gases as they disperse and attenuate sound waves. This dispersion effect can be detected by measuring the speed of sound in the gas and the corresponding absorption of sound energy. Whereas hydrocarbons have dispersion behaviors with resonances in the megahertz range, the resonance frequency of nitrogen is in the kilohertz range. By measuring the speed of sound at frequencies below, at, and above the molecular resonance frequency of nitrogen, it may be possible to directly quantify the amount of nitrogen in the natural gas sample. Measurements of frequency-dependent viscous attenuation over a spectrum of frequencies into the megahertz range may also be able to identify attenuation caused by other components of a gas sample. Alternatively, it may be possible to quantify the effective viscosity of the gas sample from such attenuation data, and correlate the viscosity to density and heating value of the gas as the fifth input quantity in the inferential algorithm. Since this approach would require fewer changes to the existing system than the microsensor approach, it is recommended for further evaluation. 


\subsection{Determination of Gas Heating Value and Energy Flow Rate}

The energy meter output is the gas heating value $\left(\mathrm{H}_{\mathrm{v}}\right)$ and energy flow rate $\left(\mathrm{Q}_{\text {energy }}\right)$ defining the amount of energy flowing in a pipeline over a particular time interval. The heating value of the gas must be measured precisely because it determines the energy content of the gas, the actual value traded in the natural gas market. The energy meter value for $\mathrm{H}_{\mathrm{v}}$ is based on measurable thermodynamic parameters in contrast with a gas chromatograph $\mathrm{H}_{\mathrm{v}}$ calculation based on the measured composition of the gas and known heating values per GPA Standard 2145-00, Revision 2 [Gas Processors Association, 2000)]. After computing the heating value, the energy flow rate is calculated by multiplying the mass-based heating value by the actual density of the gas and the actual volumetric gas flow. Thus, the accuracy of this final energy flow rate relies on the accuracy of the energy module sensors and the measurement of the pipeline flow rate.

The 2001 energy meter project endeavored to measure the heating value of gas within the allowable uncertainty limits of the algorithm, determined by uncertainties in the input parameters, namely the speed-of-sound and $\mathrm{CO}_{2}$ measurements. That is, if the speed of sound is measured to within $\pm 1.5 \mathrm{ft} / \mathrm{s}$ and the carbon dioxide concentration is measured to within \pm 0.05 mol\%, then, under the "worst-case" conditions of Table 2.9, the algorithm can compute the gas heating value to within $\pm 2.4 \mathrm{Btu} / \mathrm{scf}$ for transmission-quality gas heating values in the range of $1009 \mathrm{Btu} / \mathrm{scf}$. This uncertainty represents an error of slightly less than $\pm 0.25 \%$ in heating value.

The energy flow rate error comes from the summed root-mean-square of the heating value uncertainty and the uncertainty in flow rate, typically about $1.0 \%$ for an ultrasonic or turbine flow meter. In the case of orifice flow meters, the flow rate uncertainty is often higher, typically about $2.0 \%$. In any case, the error in $Q_{\text {energy }}$ is largely controlled by the flow rate uncertainty of $1.0-2.0 \%$. The heating value uncertainty of $\pm 0.25 \%$ has a smaller influence on the energy flow rate error. Since the accuracy in $Q_{\text {energy }}$ relies on the type of flow meter in the line, the overall uncertainty will lie between 2.0 and $2.5 \%$ for most cases.

\subsection{Laboratory Results}

The laboratory tests were divided into two parts. The first part was performed in the MRF HPL. These tests were designed to assess the ability to tap and measure high flows and high line pressures (up to 800 psia), typical of transmission sites where the module will eventually be installed. The second part was performed in the MRF LPL. At a lower line pressure of approximately 165 psia, the LPL tests spanned a range of $\mathrm{CO}_{2}$ concentrations from about 1.3 $\mathrm{mol} \%$ to $9.0 \mathrm{~mol} \%$. Since $\mathrm{CO}_{2}$ content above $3.0 \mathrm{~mol} \%$ is beyond transmission-quality standards, the LPL tests evaluated the energy meter performance with $\mathrm{CO}_{2}$ levels that might occur upstream in natural gas production environments.

In terms of heating value, the laboratory test objectives were aimed primarily at measuring $\mathrm{H}_{\mathrm{v}}$ to evaluate the uncertainty limits of the algorithm when used in conjunction with the speed-of-sound and the $\mathrm{CO}_{2}$ sensor measurements. However, to meet this target accuracy, the speed-of-sound and $\mathrm{CO}_{2}$ measurements must be within their allowable error limits as well. In the case of the HPL tests, as discussed earlier in Sections 3.2 and 3.4, the speed of sound was measured within $0.88 \mathrm{ft} / \mathrm{s}$ and the $\mathrm{CO}_{2}$ was measured within $0.05 \mathrm{~mol} \%$ after adjusting for small offset biases in the measurements. The ultrasonic speed of sound and carbon dioxide sensors performed well in the HPL tests and the results, in terms of heating value and energy flow rate 
errors, reflect those respective sensor accuracies. The LPL tests showed the $\mathrm{CO}_{2}$ sensor to have a larger measurement bias at the higher $\mathrm{CO}_{2}$ concentrations. This led to an offset in the heating value determined by the algorithm. After adjusting for the bias in the measured $\mathrm{CO}_{2}$ levels greater than $3.0 \mathrm{~mol} \%$, the heating value fell within the worst-case accuracy of $\pm 2.4 \mathrm{Btu} / \mathrm{scf}$. The scatter in the speed-of-sound and $\mathrm{CO}_{2}$ measurements was also within the uncertainty of the algorithm for a majority of the test runs.

\subsubsection{MRF HPL Tests}

The HPL line conditions were varied in each of the HPL tests to better understand the energy meter reaction to different pipeline flow rates, gas temperatures, and gas pressures. Six combinations of three line pressures (200 psig, 500 psig, and 800 psig) and two line temperatures $\left(50^{\circ} \mathrm{F}\right.$ and $\left.90^{\circ} \mathrm{F}\right)$ were tested over the course of four days. Additionally, four loop flow rates were tested, ranging from $22.2 \mathrm{ft}^{3} / \mathrm{s}$ to $3.8 \mathrm{ft}^{3} / \mathrm{s}$. The gas content within the HPL varied only slightly over the four test days and stayed within 1005 to $1007 \mathrm{Btu} / \mathrm{scf}$. The heating value results are presented in terms of heating value error, basically the difference (in Btu/scf) between the computed $\mathrm{H}_{\mathrm{v}}$ using the algorithm and the reference value. The reference $\mathrm{H}_{\mathrm{v}}$ value was obtained from the MRF gas chromatograph.

The conditions within the energy meter module during the tests were less dynamic than in the flow loop. The pressure in the speed-of-sound chamber ranged from 92 to 100 psia, while the $\mathrm{CO}_{2}$ chamber was operated at slightly above atmospheric pressure. The flow rate through the module was maintained at approximately $0.20 \mathrm{l} / \mathrm{min}$. The module temperature varied significantly with ambient conditions, ranging from $82^{\circ} \mathrm{F}$ to $125^{\circ} \mathrm{F}$. The temperature of the speedof-sound chamber and the $\mathrm{CO}_{2}$ chamber were within $1-3^{\circ} \mathrm{F}$ of each other throughout the tests.

The results of each particular set of line conditions are plotted in Figures 4.1 through 4.6. Each plot shows the heating value error and the corresponding flow rate tested at each line condition. The figures also indicate the line pressure, line temperature, and module temperature during the test. The indicated module temperature is the average temperature during each test run.

These results show that, for the majority of the test time, the heating value error fell within the worst-case $\pm 2.4 \mathrm{Btu} / \mathrm{scf}$ uncertainty for heating value. Some scatter is prevalent in the data, where the $\mathrm{H}_{\mathrm{v}}$ error exceeds the allowable uncertainty, particularly as shown in Figures 4.3 through 4.5. These irregular points may be due to the variation in the module operating conditions (caused by changing ambient temperatures throughout the test period) or the lack of stabilization time between adjustments of flow rate in the loop. The outlying points in the tests could also be due to the time differences between the flow computer and the digital data logging system being used in test. In these tests, the speed-of-sound and $\mathrm{CO}_{2}$ sensor readings were recorded every 2 minutes, whereas the reference heating values and flow rates were recorded every 3 minutes.

Figure 4.7 indicates that the average heating value error (diamonds) was less than the worst-case $\pm 2.4 \mathrm{Btu} / \mathrm{scf}$ uncertainty of the algorithm for five of the six tests. However, the error bars (indicating the standard deviation about the average) caused the heating value error to range outside of the allowable uncertainty in three of the six tests. In this regard, the energy meter module met its goal in terms of the $\mathrm{H}_{\mathrm{v}}$ accuracy in the HPL tests, particularly considering the uncorrected bias in the $\mathrm{CO}_{2}$ sensor output. 


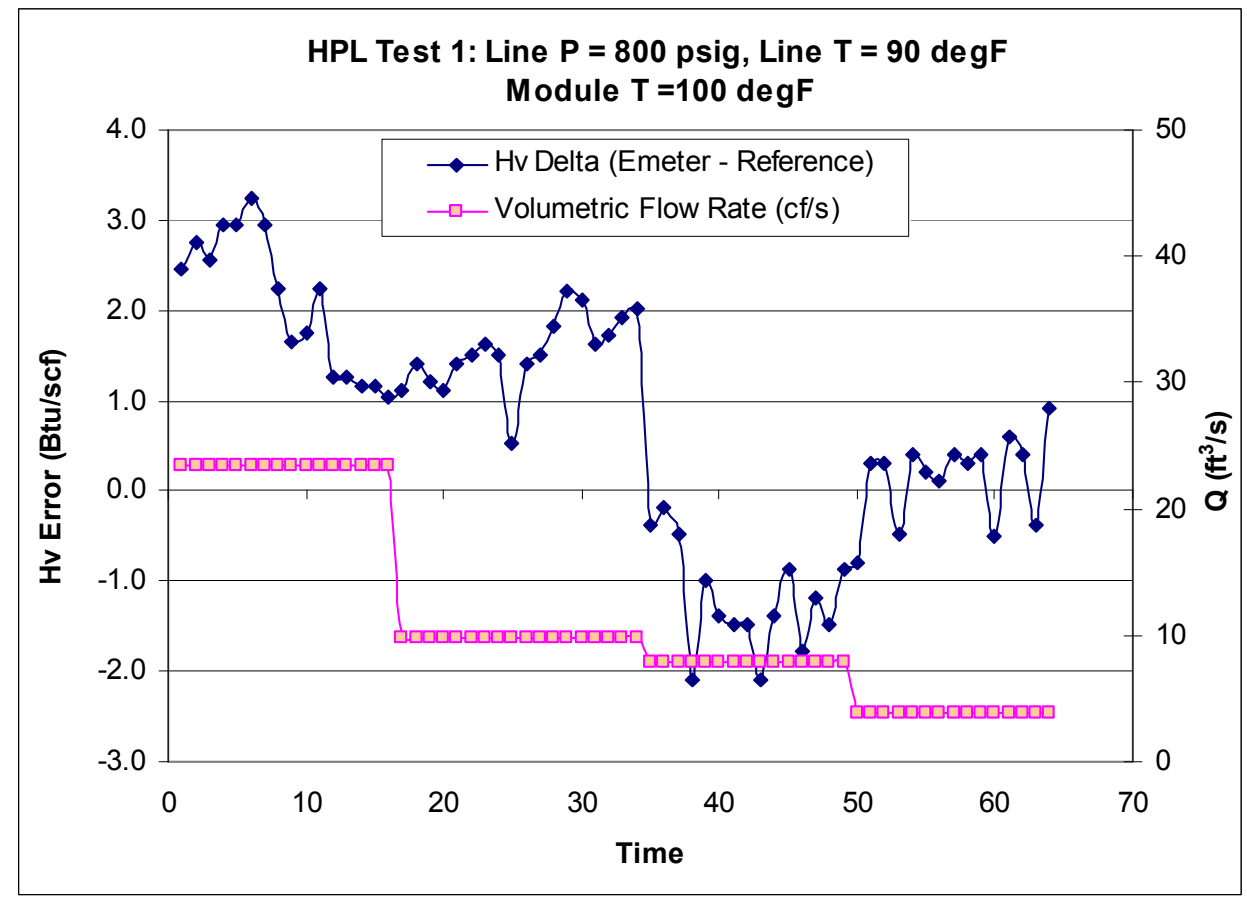

Figure 4.1. HPL Test 1 energy meter heating value error. Line pressure $=800 \mathrm{psig}$, line temperature $=90^{\circ} \mathrm{F}$.

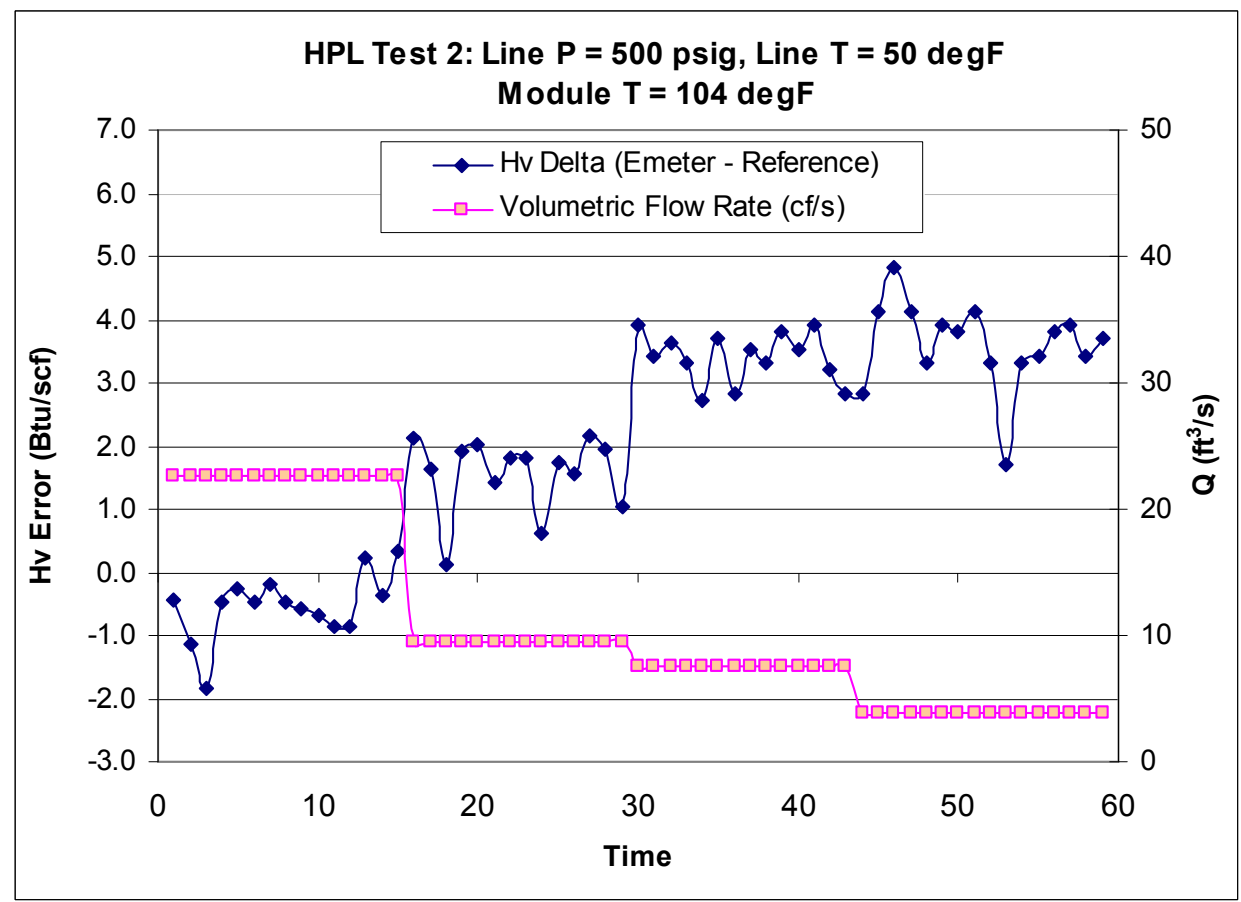

Figure 4.2. HPL Test 2 energy meter heating value error. Line pressure $=500 \mathrm{psig}$, line temperature $=50^{\circ} \mathrm{F}$. 


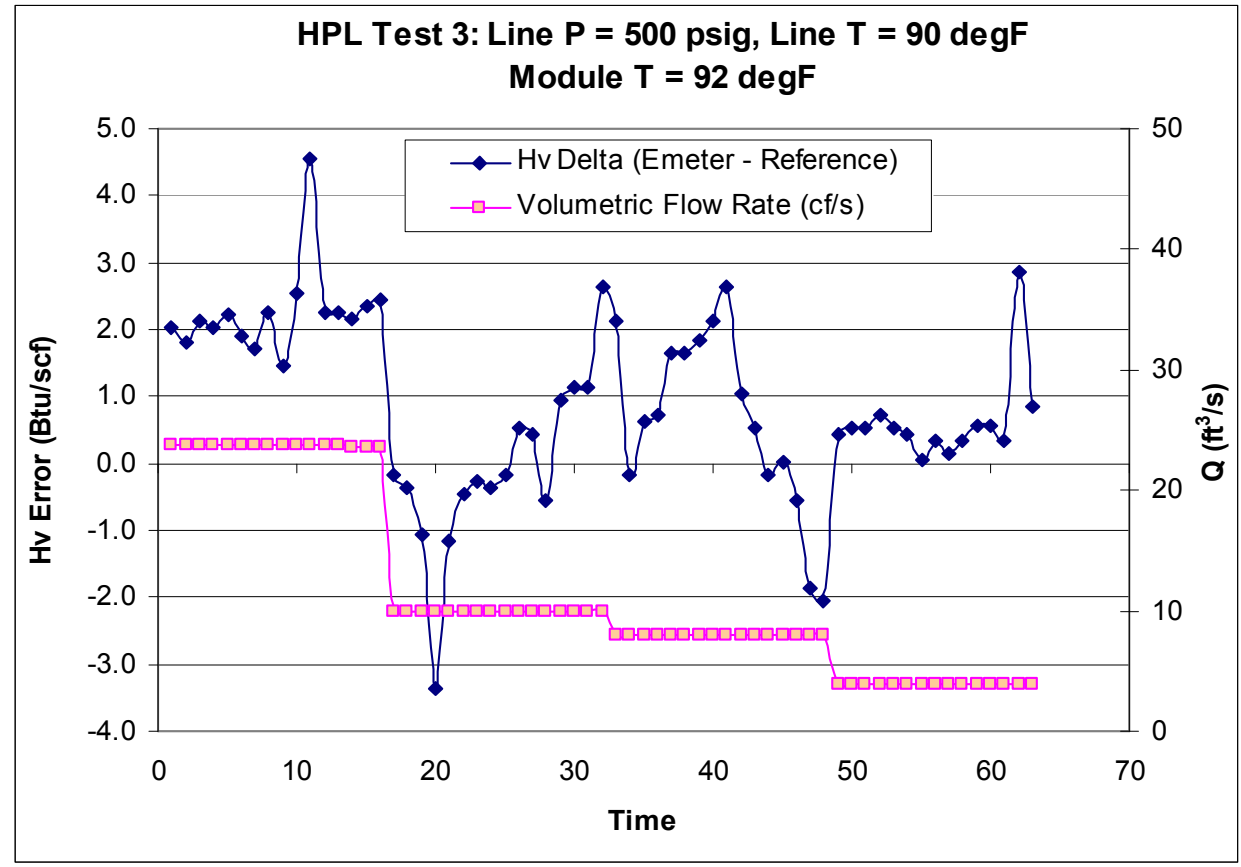

Figure 4.3. HPL Test 3 energy meter heating value error. Line pressure $=500 \mathrm{psig}$, line temperature $=90^{\circ} \mathrm{F}$.

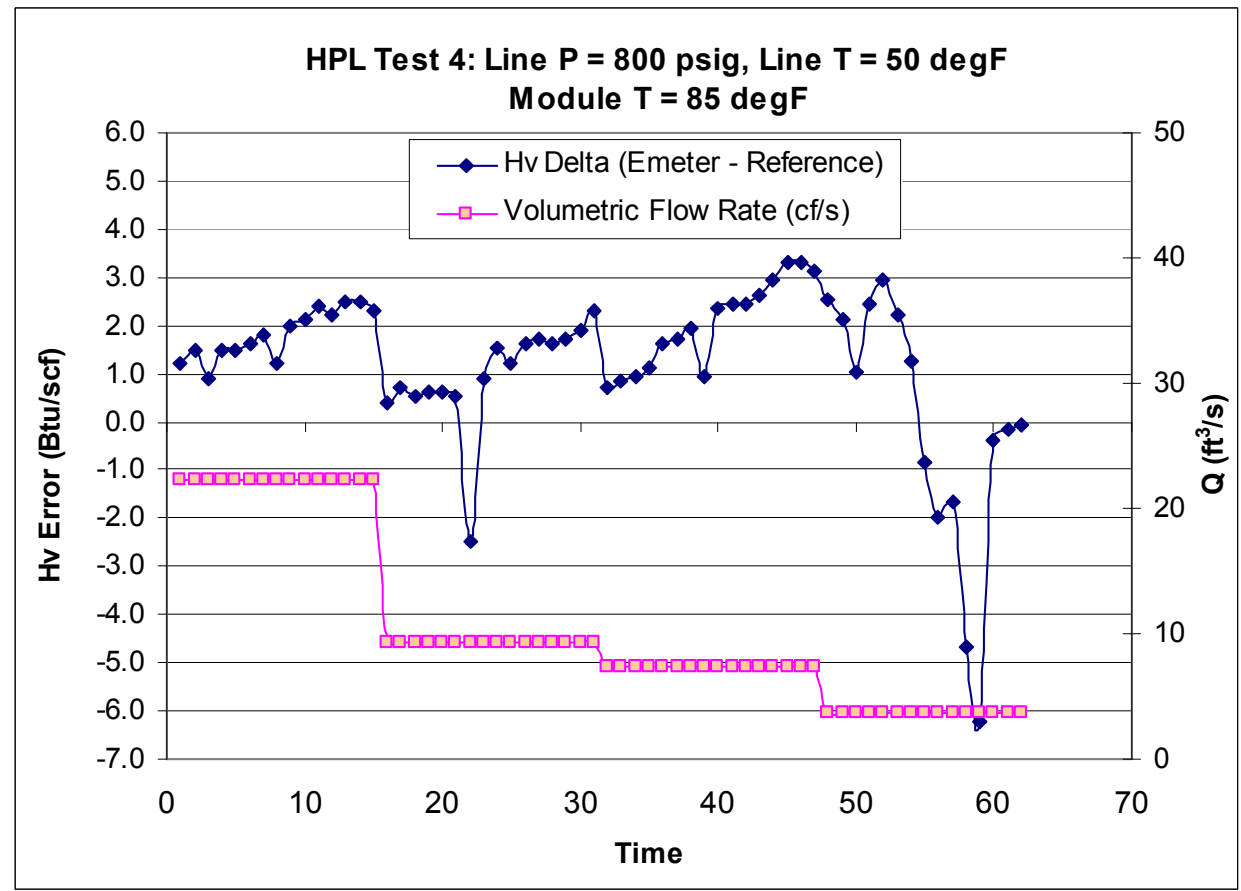

Figure 4.4. HPL Test 4 energy meter heating value error. Line pressure $=800 \mathrm{psig}$, line temperature $=50^{\circ} \mathrm{F}$. 


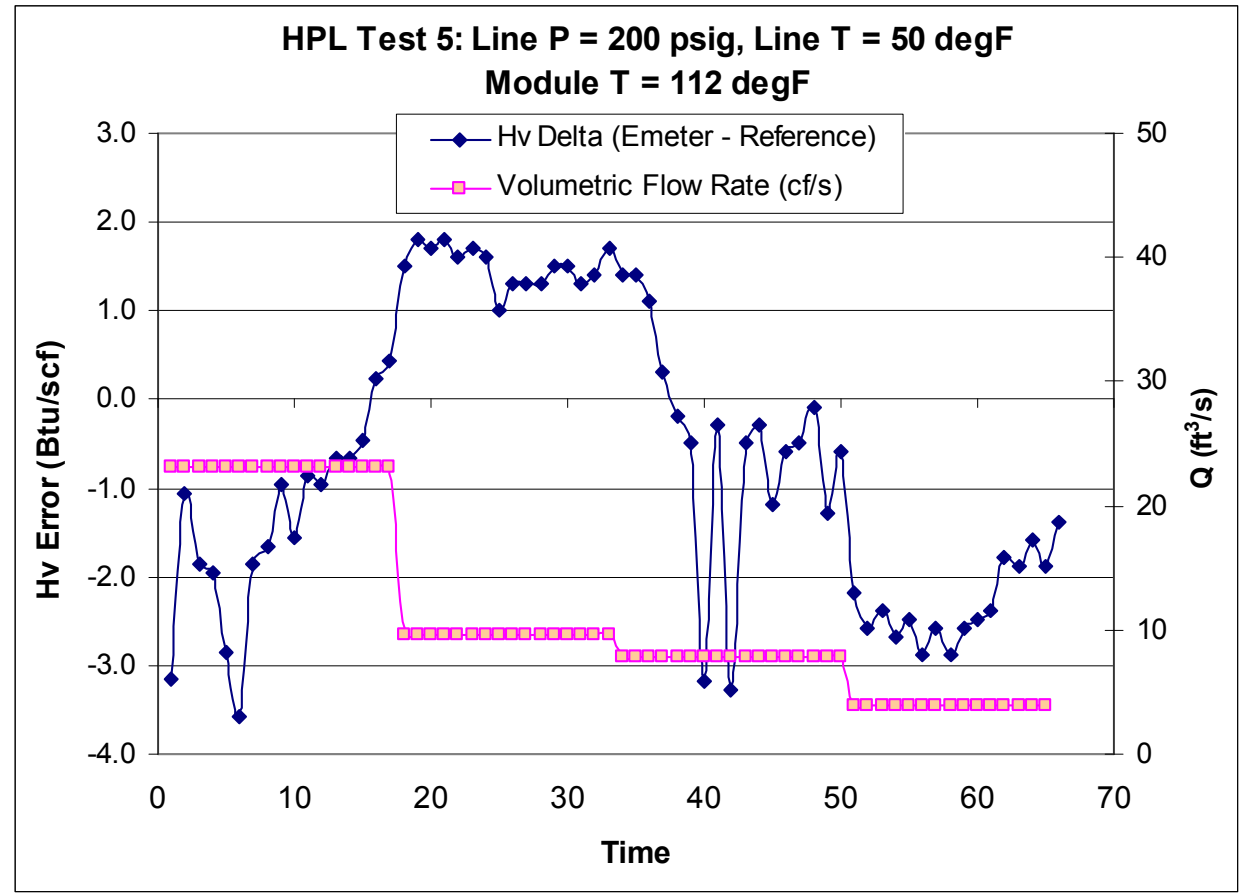

Figure 4.5. HPL Test 5 energy meter heating value error. Line pressure $=200 \mathrm{psig}$, line temperature $=50^{\circ} \mathrm{F}$.

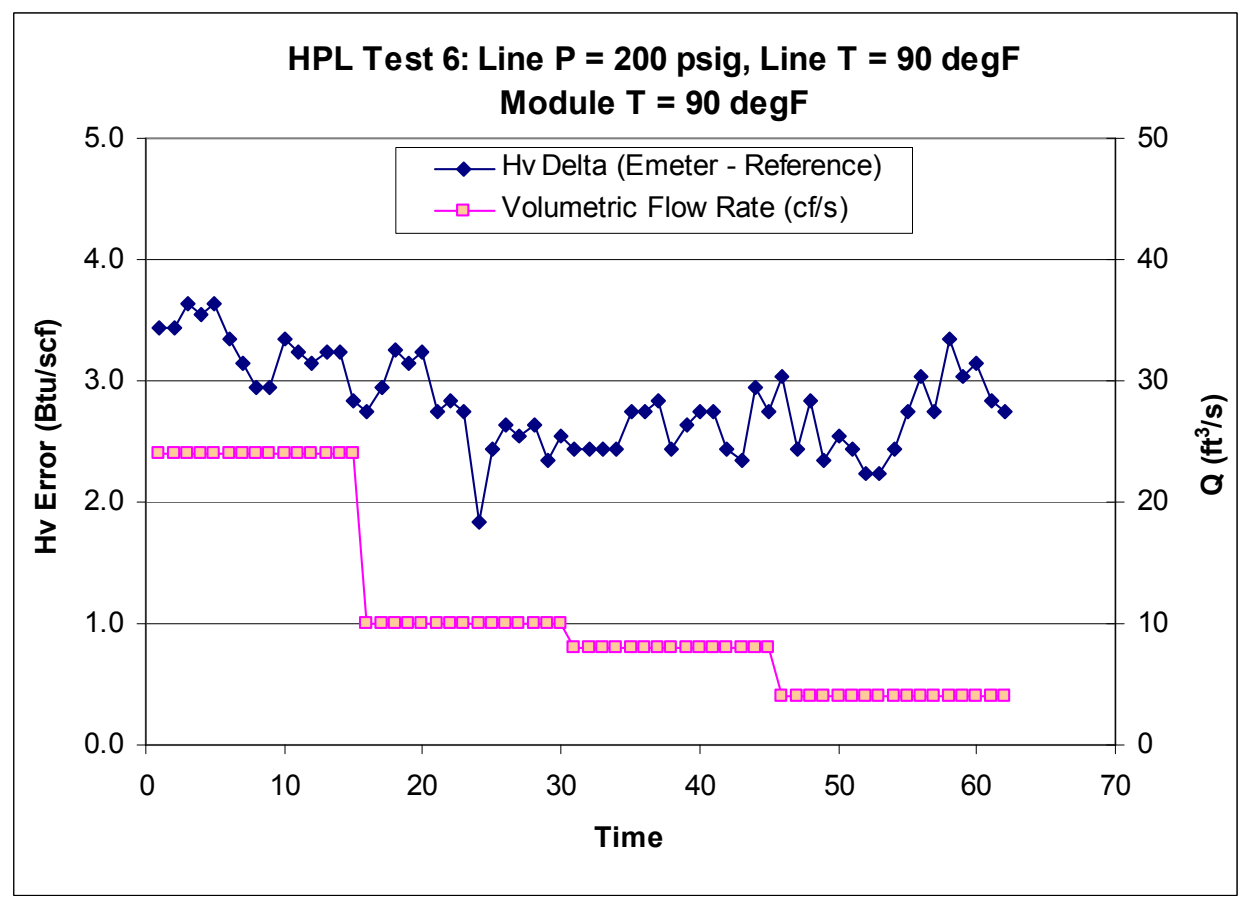

Figure 4.6. HPL Test 6 energy meter heating value error. Line pressure $=200 \mathrm{psig}$, line temperature $=90^{\circ} \mathrm{F}$. 


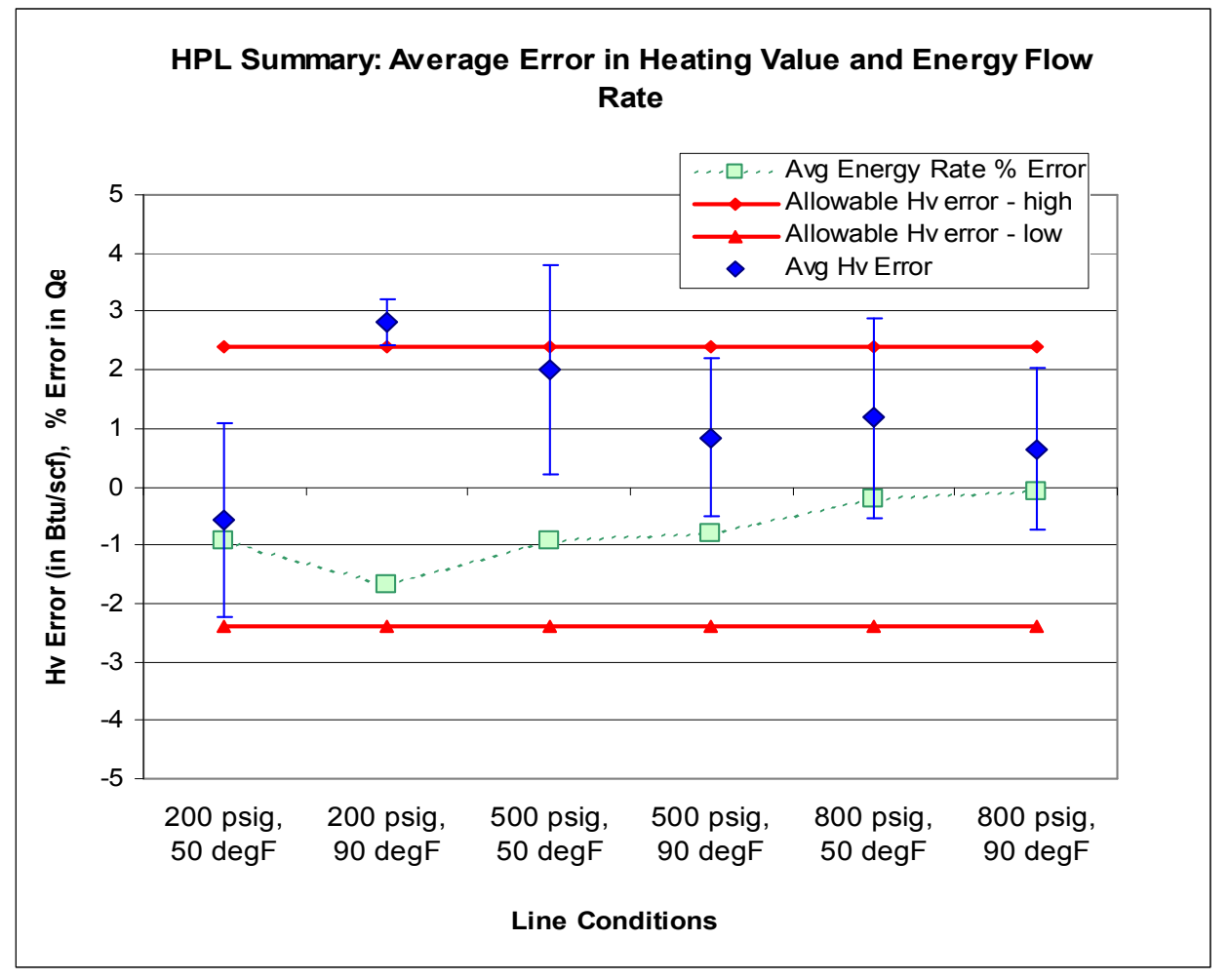

Figure 4.7. Summary of HPL tests: energy meter average heating value and average energy flow rate error.

Figure 4.7 also shows the average percentage error in Qenergy (squares). These points represent the average error in $\mathrm{Q}_{\text {energy }}$ over the four flow rates for each line condition. The $\mathrm{Q}_{\text {energy }}$ error in the laboratory tests must be applied to the heating value error since the error in actual flow rate was not previously factored into the error analysis. The figure shows the $\mathrm{Q}_{\text {energy }}$ error to be well within $2.0 \%$ in five of the six tests, indicating that the flow rate error is below $1 \%$. The decreased error at higher line pressures is due to the higher density of the gas causing the energy flow rate to increase, lowering the error percentage. Higher line pressures do not necessarily increase the magnitude of the error in energy flow rate. Assuming that the average $Q_{\text {energy }}$ error due to heating value to be approximately $1.0 \%$, the combined error using a typical line meter flow error of about $1.0 \%$ would yield a $Q_{\text {energy }}$ error of $2.0 \%-2.5 \%$.

\subsubsection{MRF LPL Tests}

The tests of the energy meter module in the LPL were aimed at evaluating the effect of a low line pressure (165 psig) and higher than normal $\mathrm{CO}_{2}$ concentrations. To test higher $\mathrm{CO}_{2}$ concentrations, typical MRF gas $\left(1009 \mathrm{Btu} / \mathrm{scf}, \mathrm{CO}_{2}=1.0 \mathrm{~mol} \%\right)$ was doped with pure $\mathrm{CO}_{2}$ to achieve increased $\mathrm{CO}_{2}$ levels of approximately $3.0 \mathrm{~mol} \%, 6.0 \mathrm{~mol} \%$ and $9.0 \mathrm{~mol} \%$. The doping procedure had the effect of lowering the hydrocarbon and nitrogen concentrations, and thus, the heating value of the gas. Throughout the tests, the line pressure was held constant and the line temperature was maintained at either $50^{\circ} \mathrm{F}$ or $80^{\circ} \mathrm{F}$. The energy meter module operating temperature was near ambient temperature, which ranged from 80 to $97^{\circ} \mathrm{F}$. Additionally, two 
operating pressures, 100 psia and 50 psia, were used in the speed-of-sound chamber, primarily to determine the speed-of-sound sensor performance at the lower pressure.

Figures 4.8 through 4.13 show the heating value error over time (diamonds) during each of the six tests performed in the LPL. The pressure in the speed-of-sound chamber is shown by the squares.

Results of the LPL tests reveal that the algorithm and module performed well in Tests 1-3, when the gas $\mathrm{CO}_{2}$ concentration was less than $3.0 \mathrm{~mol} \%$ (Figures 4.8 through 4.10). The heating value error is less than the allowable algorithm uncertainty during all of these tests. For $\mathrm{CO}_{2}$ concentrations greater than $3.0 \mathrm{~mol} \%$ in Tests $4-6$, the heating value is lower by about 10-14 Btu/scf when compared with the low $\mathrm{CO}_{2}$ concentration tests, but the error band on the derived values remains approximately within 2-4 Btu/scf. In Figures 4.11 and 4.12, one data point falls outside of this average scatter band, but these errors may have been due to transient effects within the speed-of-sound (SOS) chamber, since the data points occurred near the changeover from the higher to the lower test chamber pressure. The LPL tests also show that the speed-of-sound chamber operating pressure, although it must be accurately known, does not have a major influence on the derived heating value. The ultrasonic signal waveforms have a noticeably lower signal-to-noise ratio at 50 psia; however, the sensor appears to perform equally well at both 50 psia and 100 psia chamber pressures.

The greater error in heating value at the higher $\mathrm{CO}_{2}$ concentrations is a result of a nonlinear response in the Vaisala $\mathrm{CO}_{2}$ sensor measurements. However, as discussed in Section 3.4, this sensor can be calibrated to remove the concentration-dependent error. Figures 4.14 and 4.15 show the average heating value error plotted against line conditions (Figure 4.14) and against the line gas $\mathrm{CO}_{2} \mathrm{~mol} \%$ (Figure 4.15). From these plots, the heating value error dependence on $\mathrm{CO}_{2}$ concentration is clear. Figure 4.14 also shows the energy flow rate percentage error, which is about $4.0 \%$ over the six LPL tests. This increase in energy flow rate error, when compared with the HPL test results, is likely due to the effect of lower flow rates.

Figure 4.15 shows a linear curve fit to the average heating value error based on $\mathrm{CO}_{2}$ concentration. The equation can be applied to the energy meter module original heating values to determine the residual error (the error not attributable to the Vaisala $\mathrm{CO}_{2}$ sensor) that would result after the $\mathrm{CO}_{2}$ sensor is calibrated to correct for nonlinear response. To determine the residual error in heating value, the equation in Figure 4.15 was applied to the LPL test $\mathrm{CO}_{2}$ readings to adjust the calculated heating value. The residual error in heating value can be attributed to the other inputs to the algorithm and is shown in Figure 4.16.

After applying the correction for the $\mathrm{CO}_{2}$ sensor, the LPL heating value averages fall within the target error for heating value, though the standard deviation (shown by the error bars) lies outside of the error band in the $6.5-10 \mathrm{~mol} \% \mathrm{CO}_{2}$ range. Based on these results, the LPL tests revealed that the algorithm and energy meter module can perform fairly accurately in a nontransmission-quality (high diluents) type of natural gas. With additional refinement of the module sensors and the addition of an improved $\mathrm{CO}_{2}$ sensor calibration process, the energy meter module will be capable of measuring heating values and energy flow rates to an acceptable degree of accuracy under a wide range of diluent gas concentrations. 


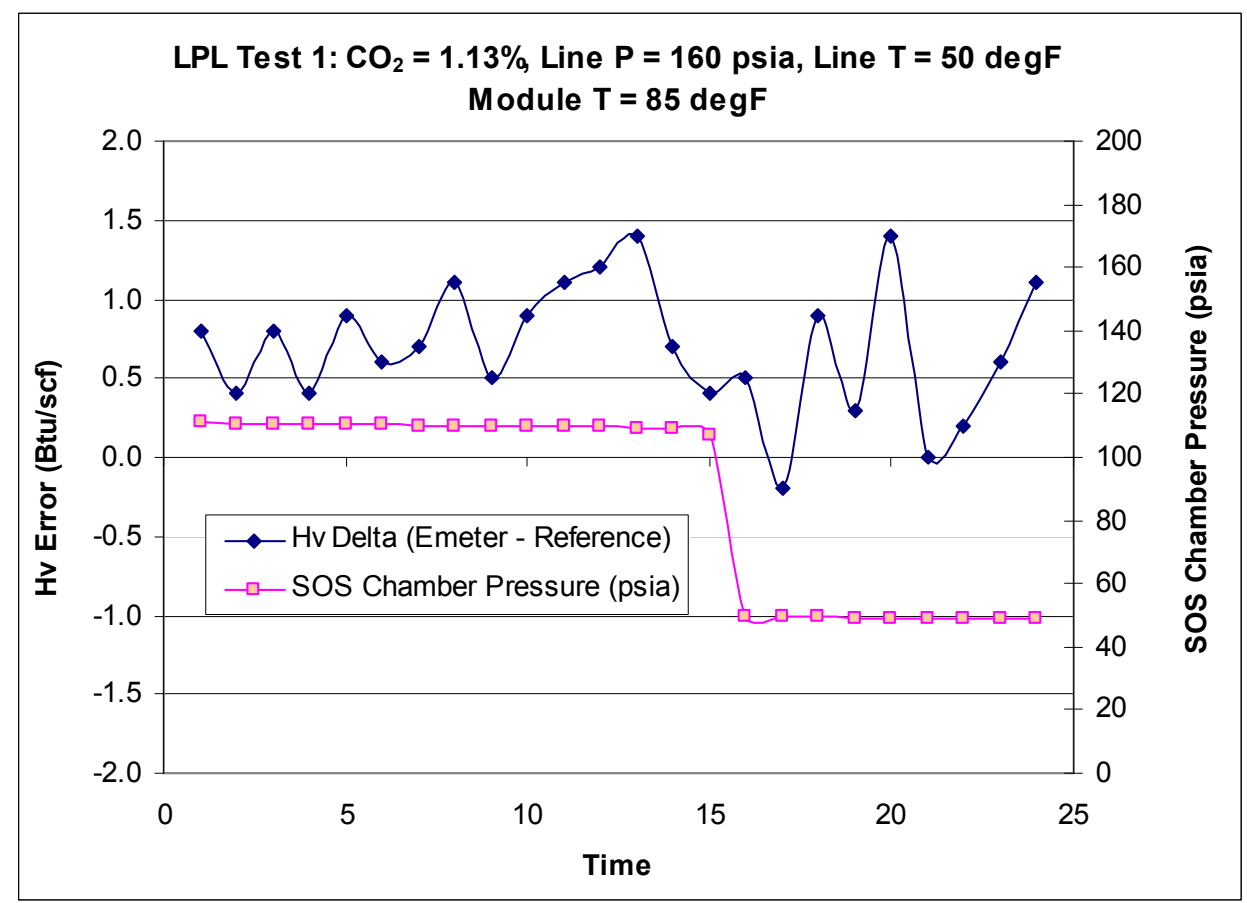

Figure 4.8. LPL Test 1: energy meter heating value error at line $\mathrm{T}=50^{\circ} \mathrm{F}$ and $\mathrm{CO}_{2}=1.1 \%$.

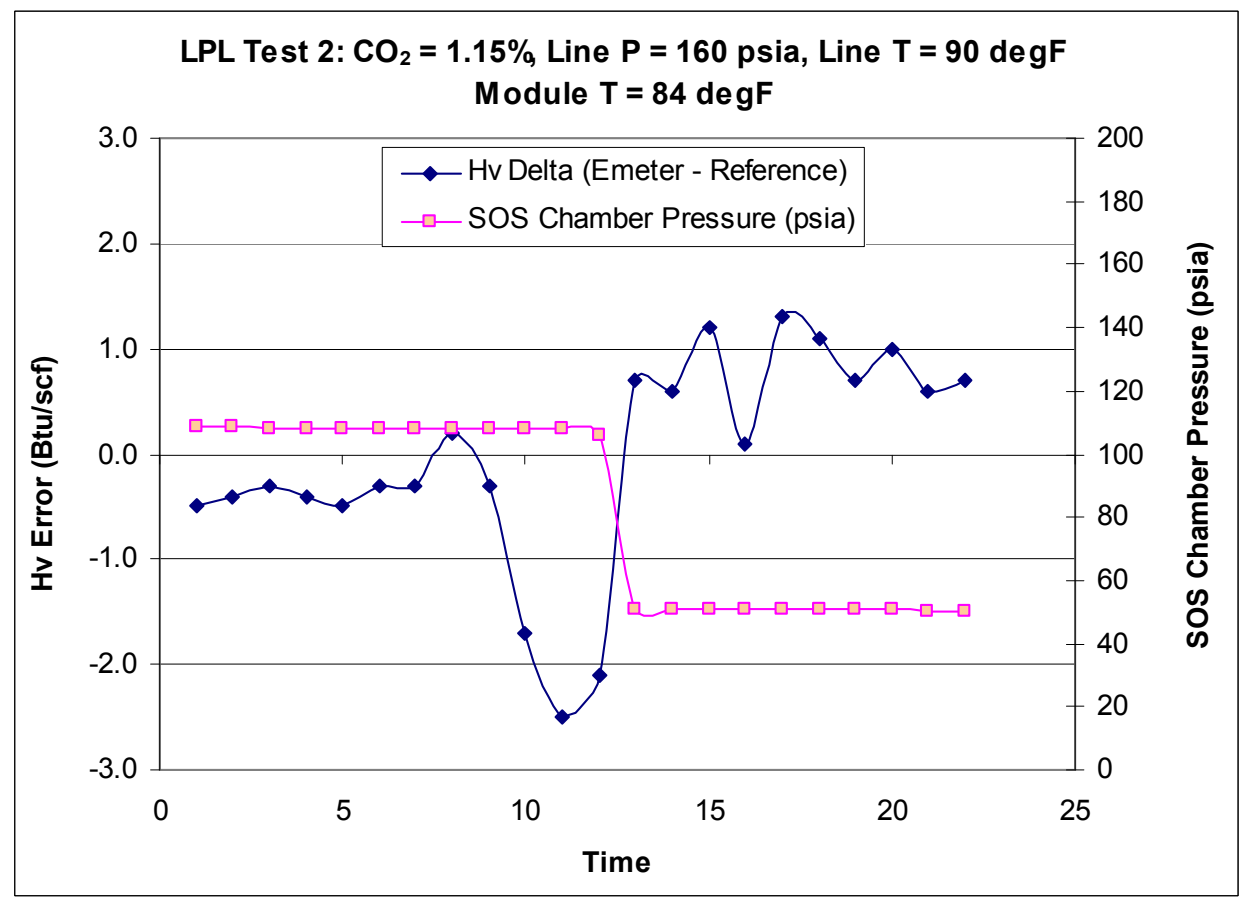

Figure 4.9. LPL Test 2: energy meter heating value error at line $\mathrm{T}=90^{\circ} \mathrm{F}$ and $\mathrm{CO}_{2}=1.15 \%$. 


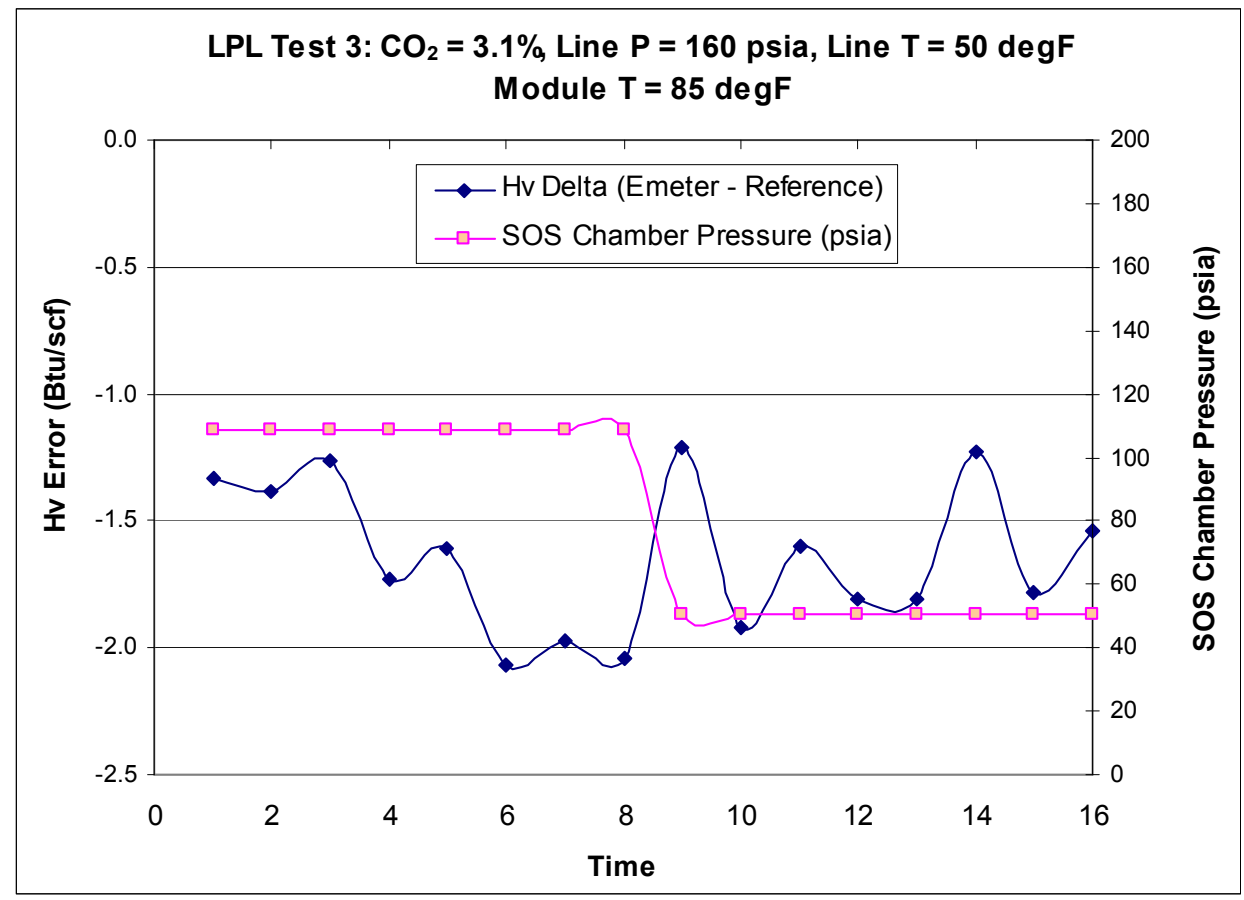

Figure 4.10. LPL Test 3: energy meter heating value error at line $\mathrm{T}=50^{\circ} \mathrm{F}$ and $\mathrm{CO}_{2}=3.10 \%$.

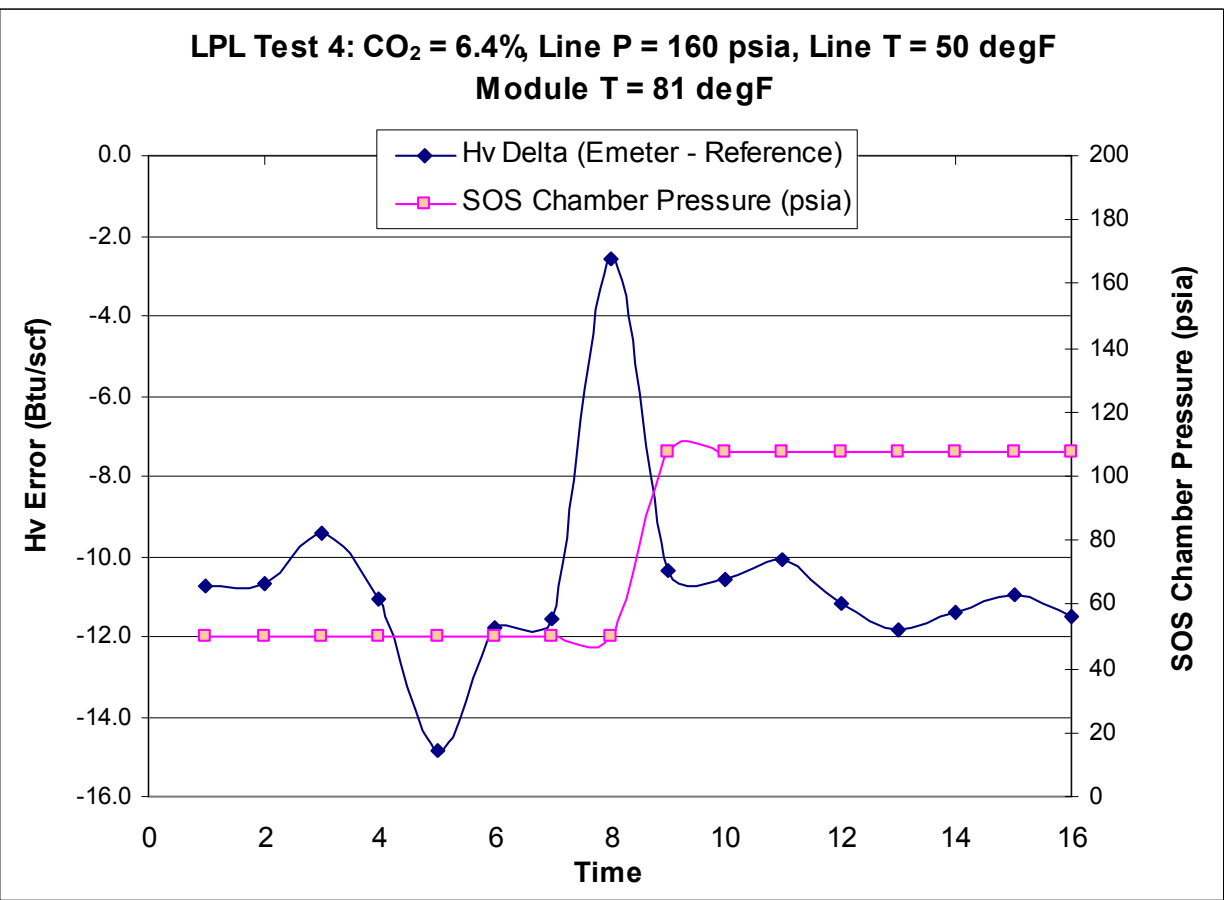

Figure 4.11. LPL Test 4: energy meter heating value error at line $\mathrm{T}=50^{\circ} \mathrm{F}$ and $\mathrm{CO}_{2}=6.40 \%$. 


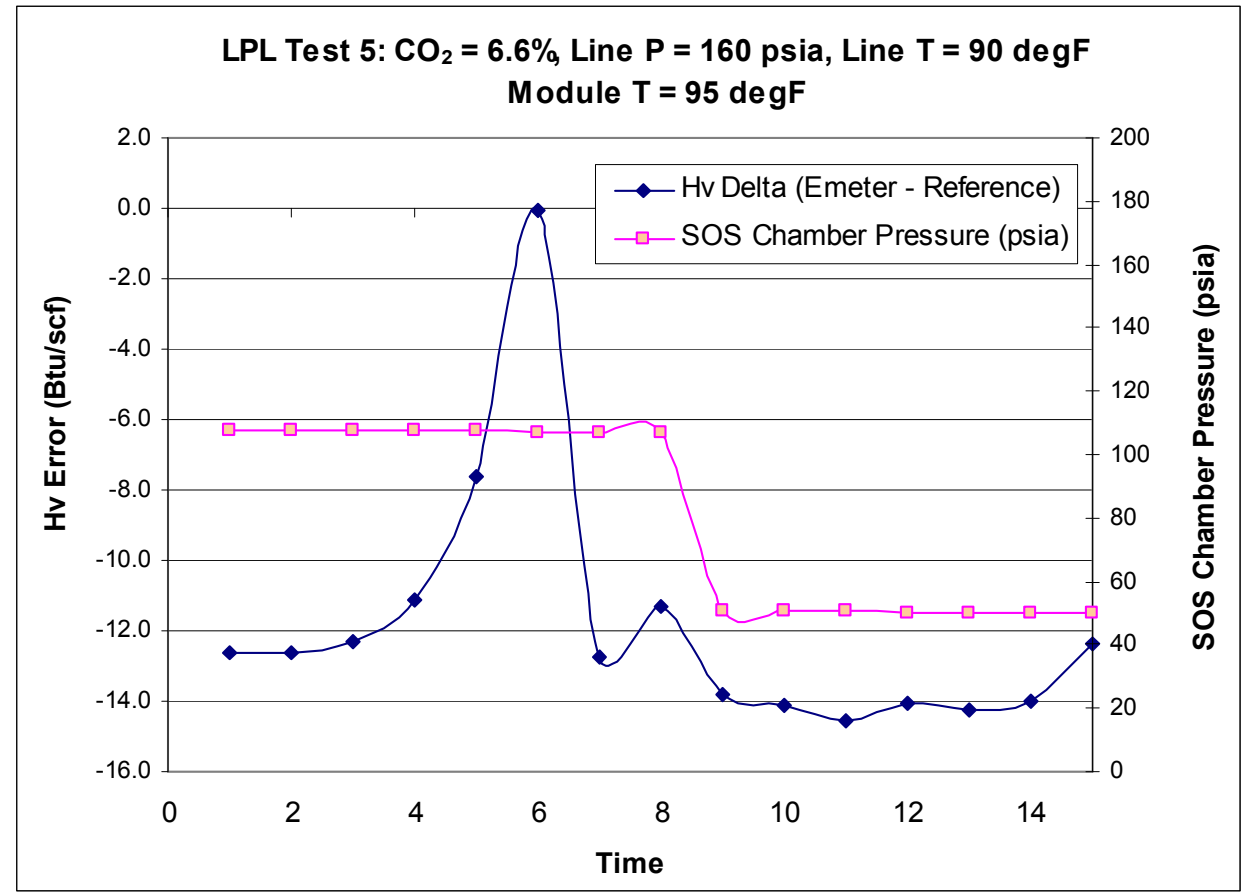

Figure 4.12. LPL Test 5: energy meter heating value error at line $\mathrm{T}=90^{\circ} \mathrm{F}$ and $\mathrm{CO}_{2}=6.60 \%$.

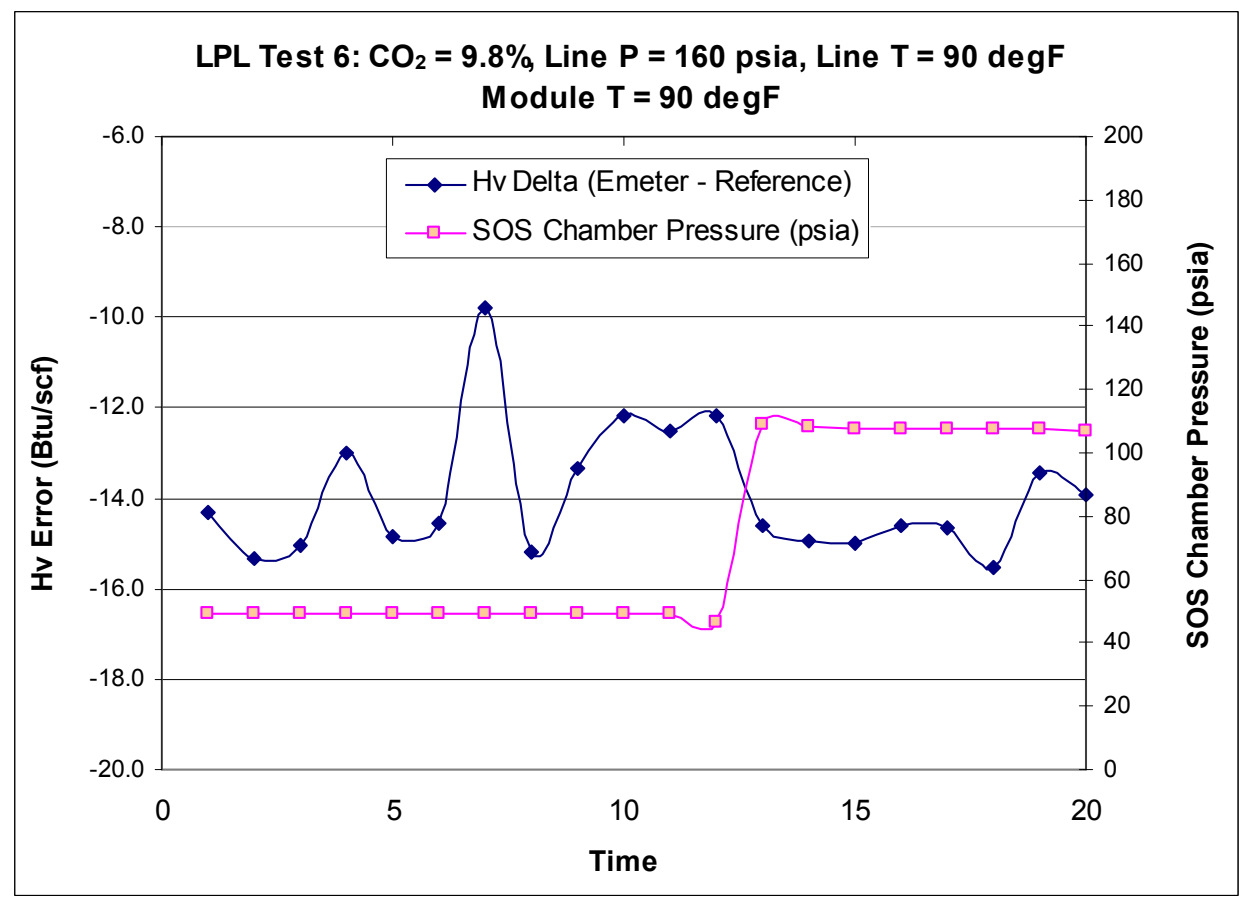

Figure 4.13. LPL Test 6: energy meter heating value error at line $\mathrm{T}=90^{\circ} \mathrm{F}$ and $\mathrm{CO}_{2}=9.80 \%$. 


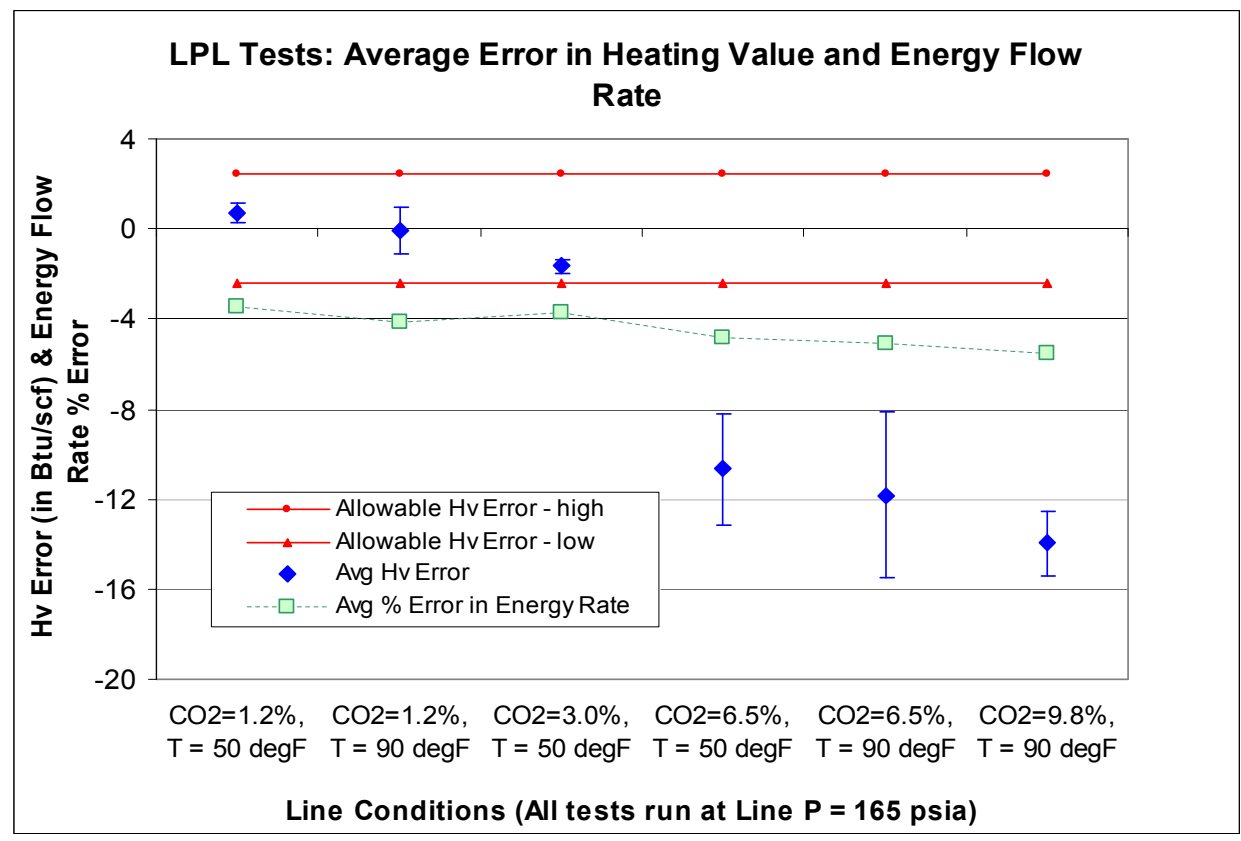

Figure 4.14. Summary of LPL tests: energy meter average heating value and average energy flow rate error.

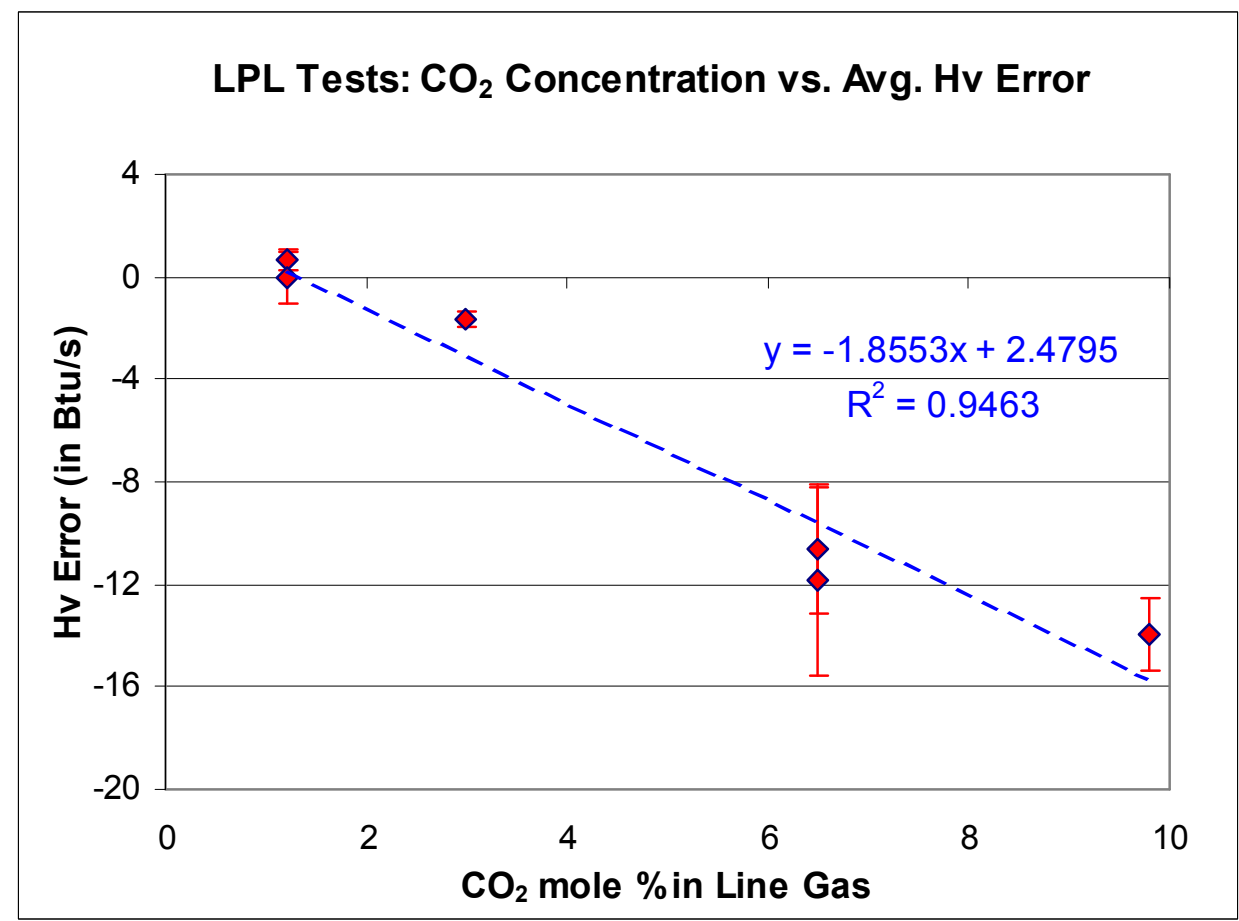

Figure 4.15. LPL tests: correlation between $\mathrm{CO}_{2}$ concentration (in mol\%) and $\mathrm{H}_{\mathrm{v}}$ error (in Btu/scf). 


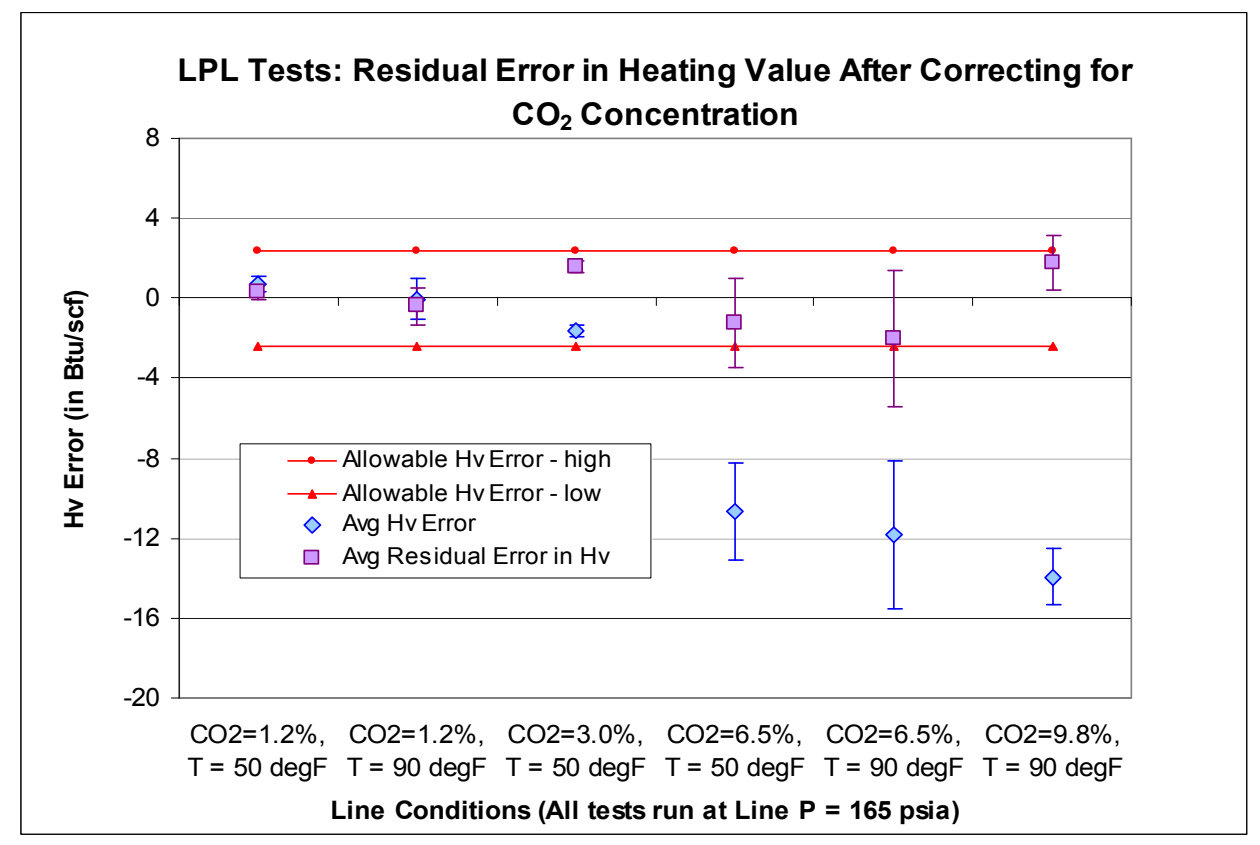
Figure 4.16. Summary of LPL tests: energy meter average heating value
and average energy flow rate error.

\subsection{Field Tests and Results}

Field tests performed during the 2001 project served as an extension of the laboratory tests by validating the energy meter algorithm in a more variable "real world" setting. The field site was located at an inlet to a gas-fired power plant and, thus, its line conditions and energy flow rates were more dynamic than those previously observed in the laboratory tests. Line temperature ranged from $41-76^{\circ} \mathrm{F}$, pressure from $590-647 \mathrm{psia}, \mathrm{CO}_{2}$ from $0.4-1.35 \mathrm{~mol} \%$, and $\mathrm{N}_{2}$ from $0.5-1.82 \mathrm{~mol} \%$. By comparing the heating value from the field site gas chromatograph with the heating value determined by the energy meter algorithms, the effect of fluctuating temperatures, pressures, and diluent concentrations could more easily be seen. Additionally, the field testing provided improved guidelines for future field testing through a better understanding of field site requirements for the energy meter and data recording.

In order to record field test data over a two-week period from multiple flow computers and a gas chromatograph, an RGC 2000 digital data logger instrument was used. Manufactured by Integrated Information Technologies, this data logger is compatible with several different drivers and capable of accepting multiple communication protocols and parameters. In the 2001 field tests, the data logger was configured to record the gas chromatograph compositions, the energy meter algorithm results, and flow data from the field site ultrasonic meter. The data logger was programmed to record data every five minutes over the entire two-week test period.

During this first phase of field tests, only the energy meter algorithm was tested. Since the energy meter module, containing the speed-of-sound sensor and carbon dioxide sensor, was not included in these tests, the algorithm performance was independent of the energy meter module effects. Instead of measuring $\mathrm{CO}_{2}$ and $\mathrm{N}_{2}$ concentrations, which are required as inputs to 
the algorithm, the $\mathrm{CO}_{2}$ and $\mathrm{N}_{2}$ levels were held fixed at a constant value. After the data were recorded with these diluent concentrations set at particular values, the observed and recorded $\mathrm{CO}_{2}$ and $\mathrm{N}_{2}$ concentrations were entered into the algorithm and the new results compared. The speed of sound, line temperature, and line pressure (the remaining three inputs to the algorithm) were obtained from an ultrasonic flow meter and pressure and temperature transducers, respectively.

Figure 4.17 shows a data flow diagram from the flow meters to the field devices and the data logger. The first Bristol Babcock flow computer (BB\#1) was programmed with the energy meter algorithm and received its speed-of-sound signal from the Line 2 ultrasonic flow meter (USFM2) via Modbus. It also received analog signals for pressure and temperature from transmitters installed on the same line. The BB\#1 then computed the heating value, molecular weight, and gas density using the algorithm. The computed values were fed to the data logger using Bristol Babcock's protocol.

The second Bristol Babcock flow computer (BB\#2) received the flow rate signals from all three of the inlet lines to the gas plant. By tapping into this flow computer, the individual line (volumetric) flows as well as the total station flow could be recorded as reference values. The $\mathrm{BB} \# 2$ also gave the USFM 2 temperature and pressures for Line 2, which was being sampled by the algorithm.

The field site Daniel 2350 gas chromatograph was connected to the data logger as the third field device. Data on gas composition, saturated and unsaturated heating value, and the unnormalized total were used as the final reference for comparison against the energy meter algorithm. Figure 4.18 shows the three field devices being sampled by the data logger set up in an insulated field office. The data logger was operated continuously during the testing.

The data logger results revealed that the algorithm performed well in computing the heating value, given the dynamic conditions at the field site. The initial $\mathrm{CO}_{2}$ and $\mathrm{N}_{2}$ concentrations were set based on their actual values obtained from the gas chromatograph on the first day of logging. However, these values, as revealed in Figure 4.19, were not typical of the entire two-week period. The two-week period represented by these plots actually began two days after the initial $\mathrm{CO}_{2}$ and $\mathrm{N}_{2}$ levels were set. Thus, the average $\mathrm{CO}_{2}$ concentration is approximately $0.60 \mathrm{~mol} \%$, roughly $0.20 \%$ less than the constant value set in the algorithm. Conversely, the average $\mathrm{N}_{2}$ concentration is approximately $1.40 \mathrm{~mol} \%$, roughly $0.30 \%$ higher than the value used in the algorithm. The partially compensating effects of the low $\mathrm{CO}_{2}$ and high $\mathrm{N}_{2}$ values cannot be expected in all cases; thus it is possible for both values to run high or low, which would cause the heating value to be shifted further from its reference value.

Power plant operations normally shut down gas supply Line 2 being monitored by the algorithm at low demand hours, typically between midnight and $7 \mathrm{am}$ on weekdays. These periods of low flow distorted the data somewhat, because the temperature of the line dropped dramatically to ambient conditions when gas was not flowing in the line. However, the algorithm handled the temperature drop and corresponding changes in speed of sound very well. As shown in Figure 4.20, the temperature drops are daily occurrences and easily explained by corresponding periods of no flow. Figure 4.21 shows the pressure readings from the pressure transmitter (the energy meter value) and the USFM pressure value (the reference value). Line pressure is more stable in its average about the mean, and the energy meter value is typically 1.2 psia, or roughly $0.2 \%$, higher than the USFM value. 


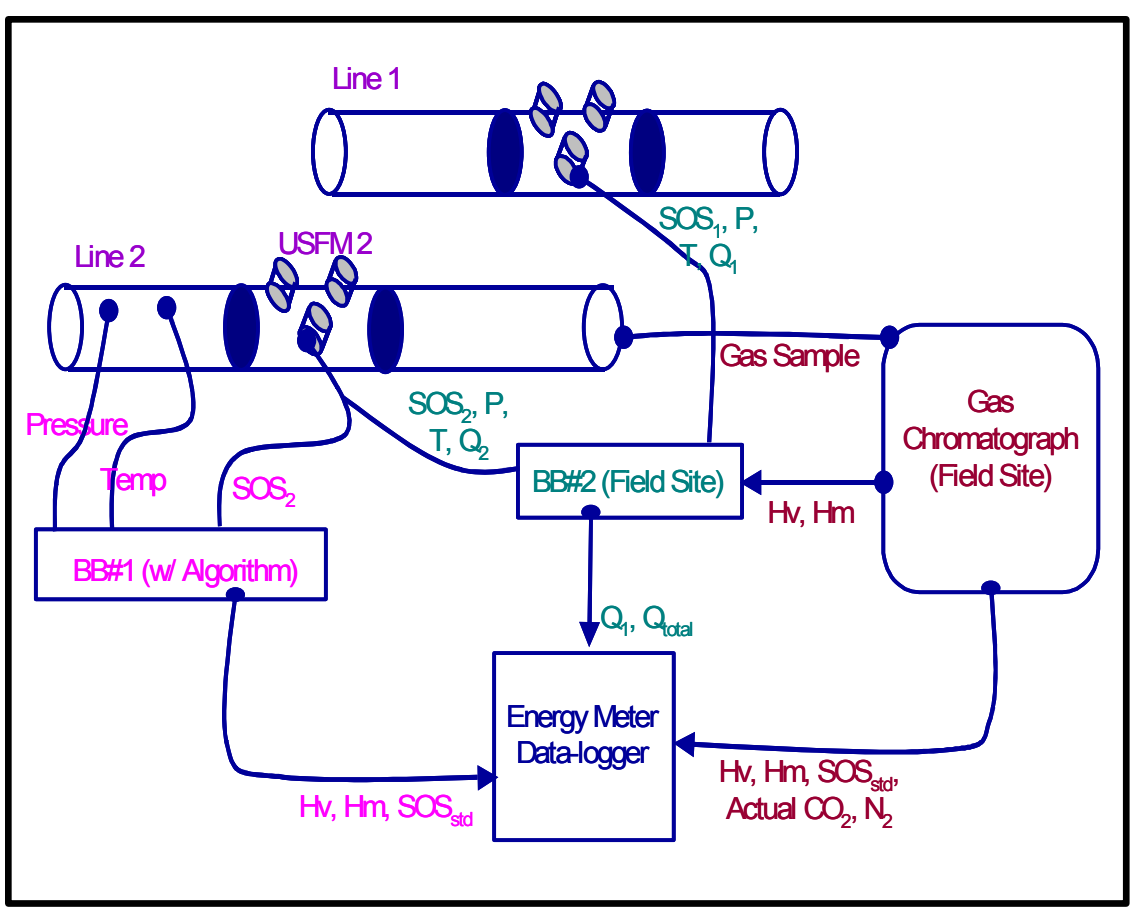

Figure 4.17. Configuration of field devices for energy meter algorithm test.
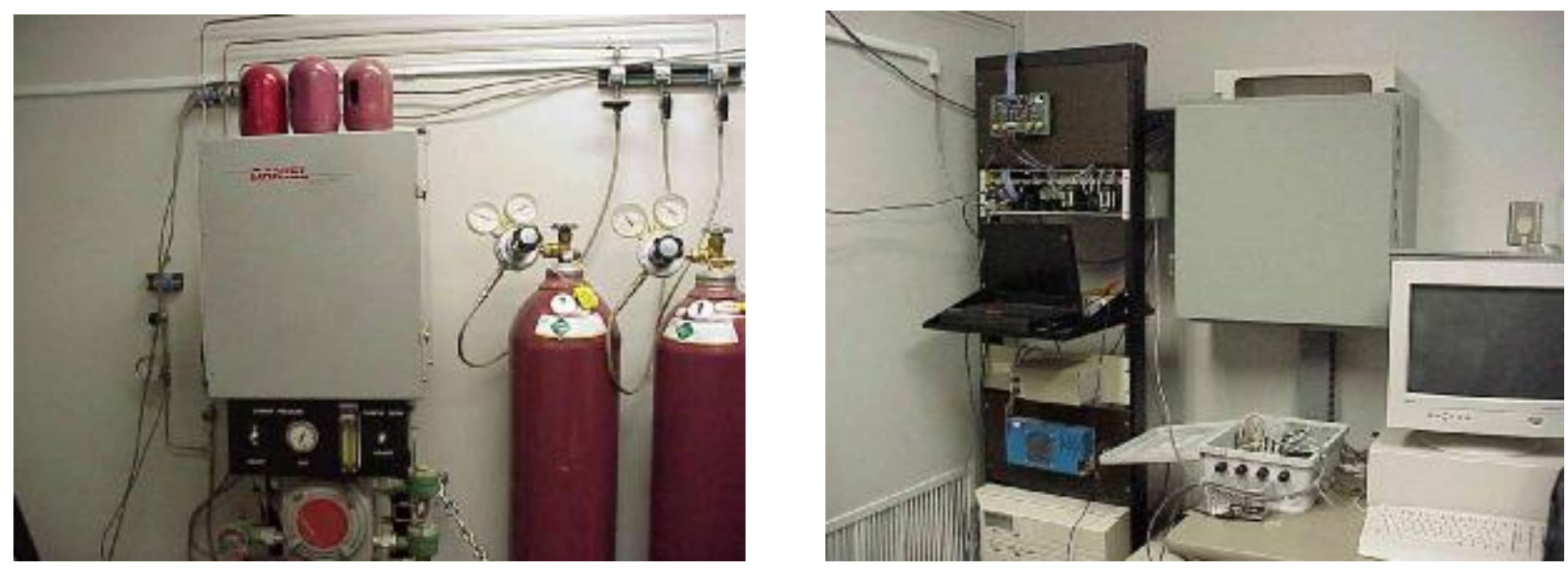

Figure 4.18. Field site devices: Daniel 2350 gas chromatograph (left) and flow computer rack (right) with two Bristol Babcock computers, at top and bottom. 


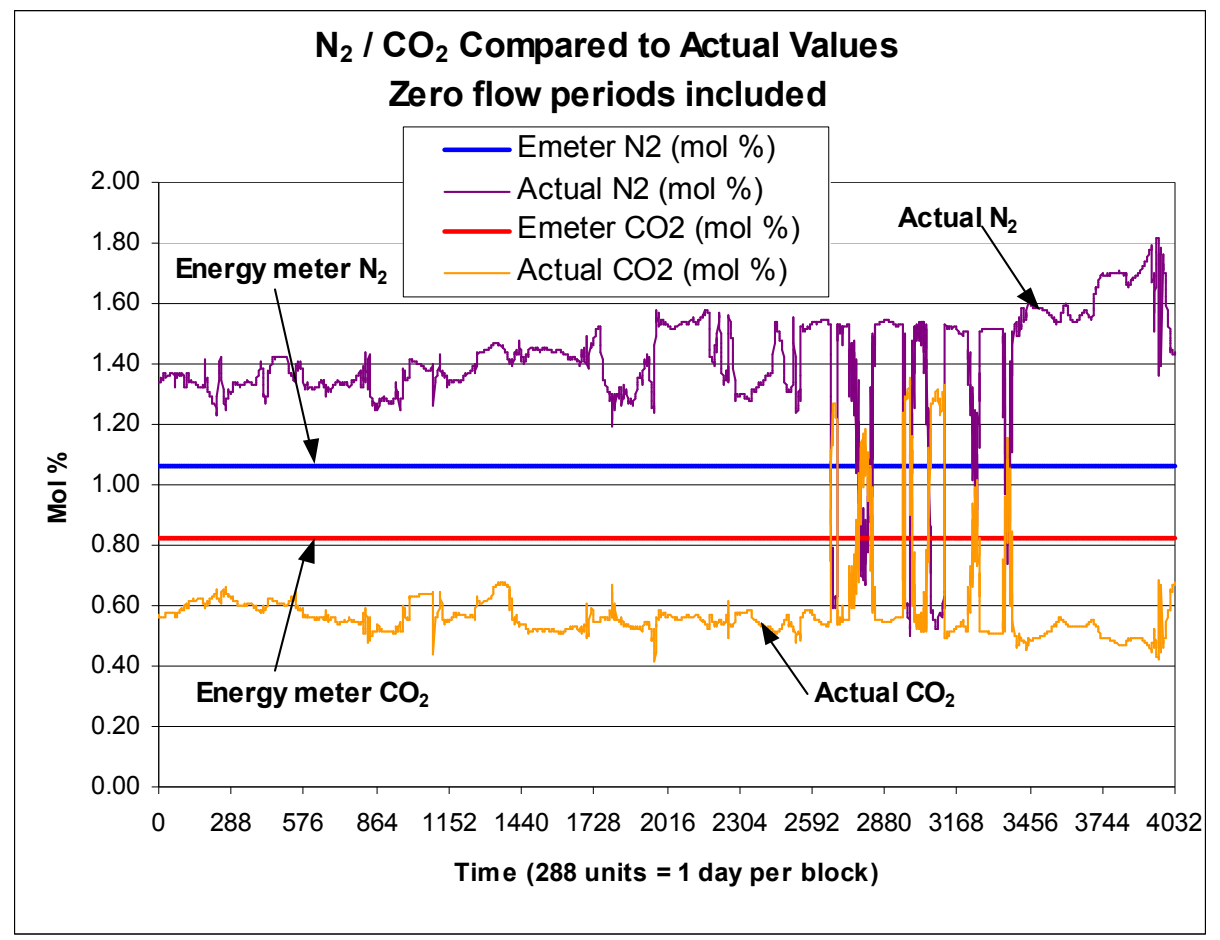

Figure 4.19. Energy meter $\mathrm{CO}_{2}$ and $\mathrm{N}_{2}$ values compared to actual concentrations.

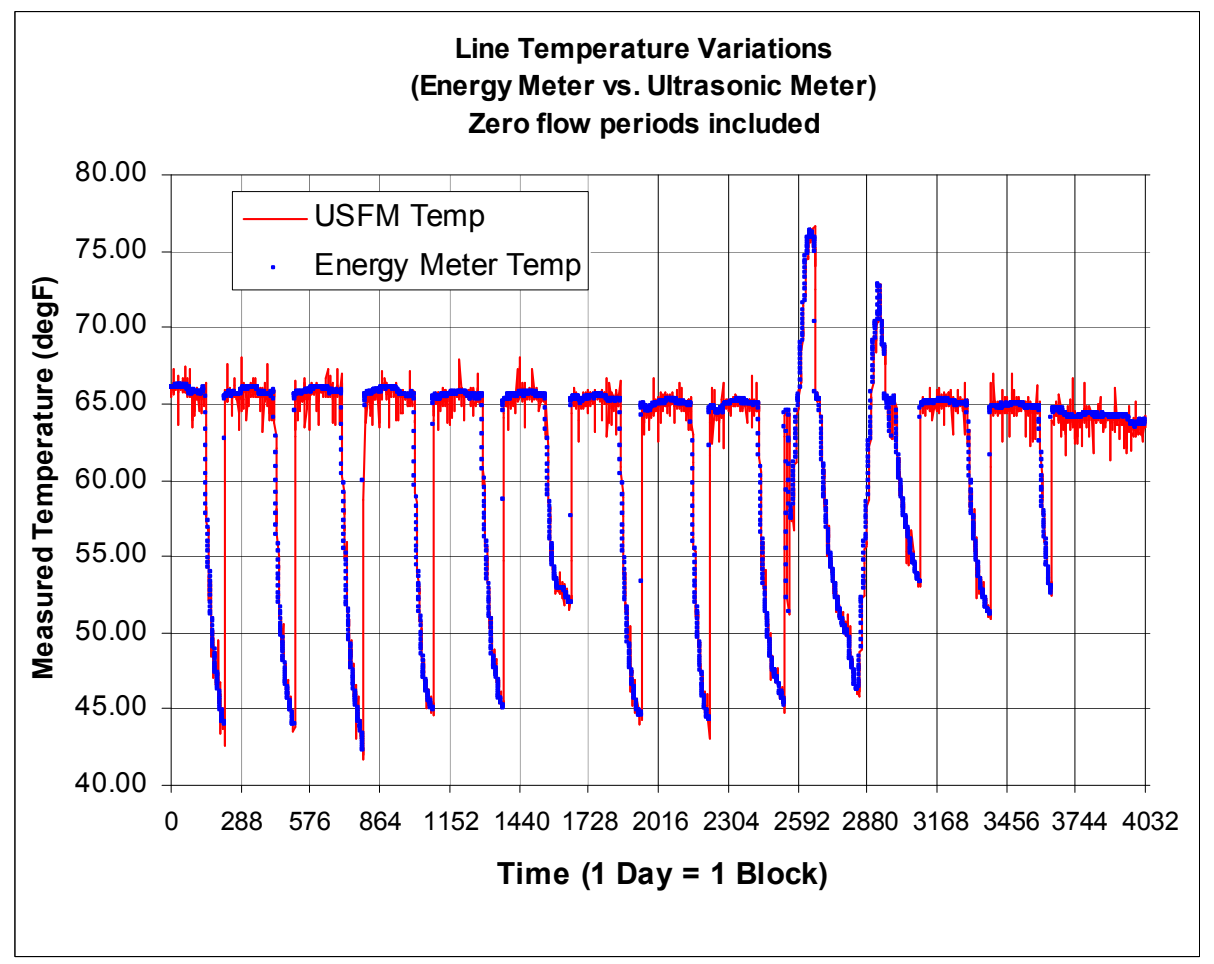

Figure 4.20. Energy meter line temperature compared to USFM2 temperature. 


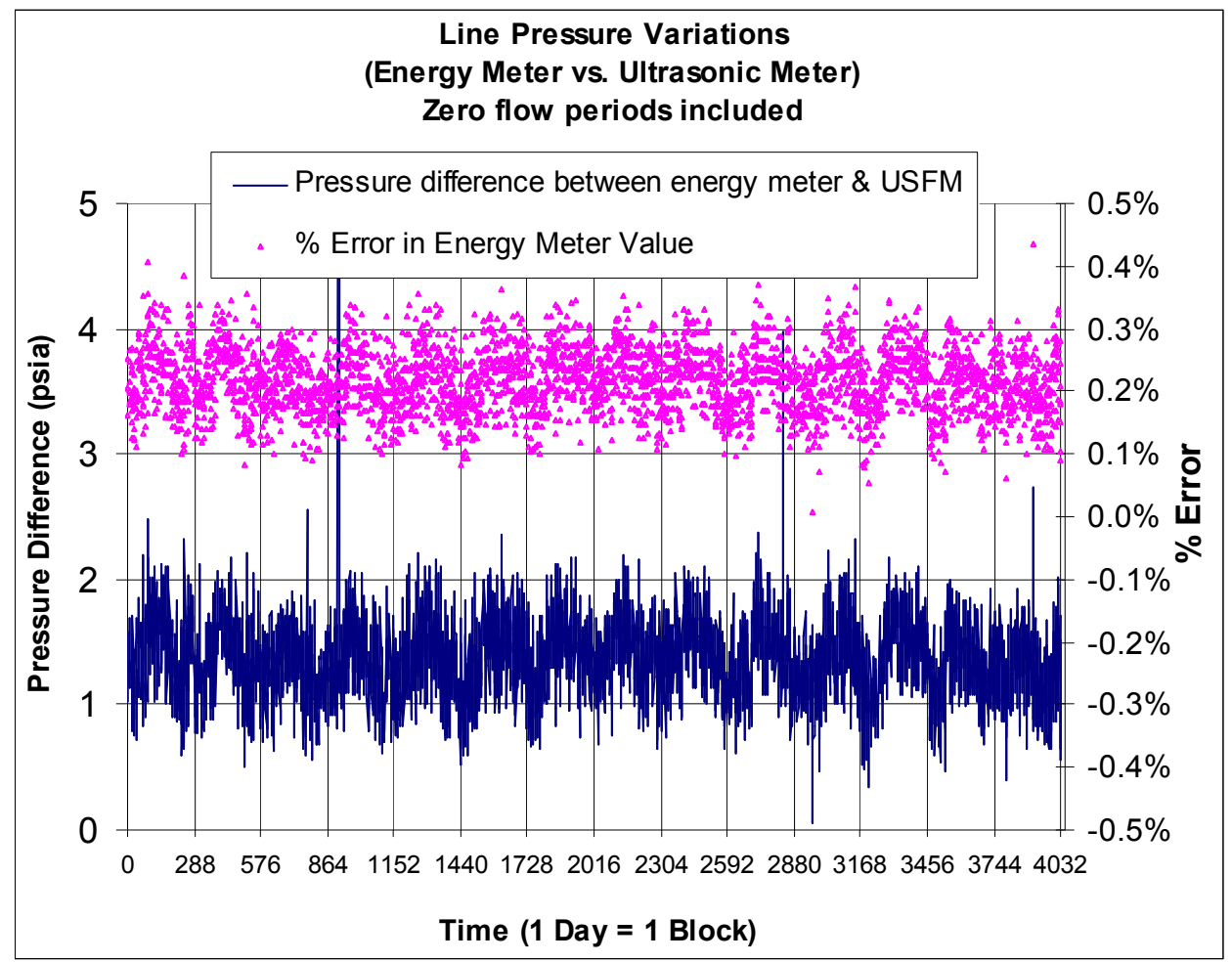

Figure 4.21. Energy meter line pressure compared to USFM2 pressure.

The main distortions due to "no flow" periods occurred when the line was dramatically re-opened in the morning hours and a surge of gas (typically at temperatures $30^{\circ} \mathrm{F}$ higher than ambient) raised the line temperature and flow within a period of one minute or less. Since the field devices were not synchronized by the minute and the conditions were changing very quickly, the difference in recorded values for speed of sound, temperature and, in turn, derived heating value were more pronounced at times when flow was coming back into the line. Because of incompatibility in the data caused by the non-synchronized data recording devices, the periods of "no flow" and surging flow were removed in examining the actual heating value errors.

Figure 4.22 shows the energy meter computation of heating value, assuming constant initial $\mathrm{CO}_{2}$ and $\mathrm{N}_{2}$ concentrations. Obviously, the heating value error is considerable due to the fact that the algorithm did not account for the variations in $\mathrm{CO}_{2}$ and $\mathrm{N}_{2}$ concentrations. However, the agreement is within $\pm 10 \mathrm{Btu} / \mathrm{scf}$ with respect to the gas chromatograph, and is within 5 $\mathrm{Btu} / \mathrm{scf}$ for most of the test period.

Adjusting the algorithm inputs to mirror the actual varying concentrations of $\mathrm{CO}_{2}$ and $\mathrm{N}_{2}$ (as determined by the gas chromatograph) significantly reduced the heating value error. Figure 4.23 shows the adjusted energy meter heating value that was determined by running the algorithm again using the on-line gas chromatograph $\mathrm{CO}_{2}$ and $\mathrm{N}_{2}$ concentration levels. This graph also provides a check on the algorithm, since all of the input parameters to the algorithm were measured using the reference instruments. The difference between the gas chromatograph and the energy meter algorithm averaged $-0.41 \mathrm{Btu} / \mathrm{scf}$, with a standard deviation of 0.90 


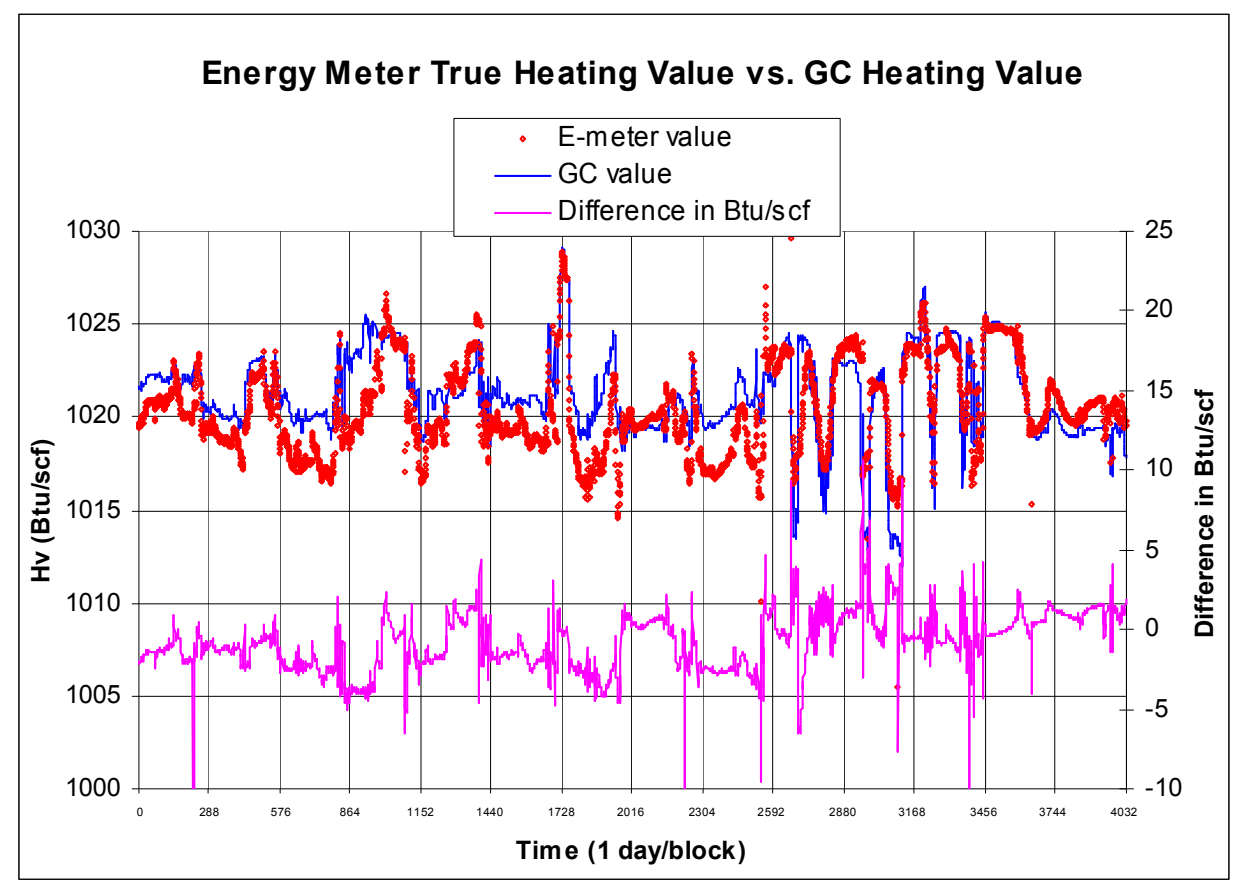

Figure 4.22. Comparison of algorithm heating value determination at constant $\mathrm{CO}_{2}$ and $\mathrm{N}_{2}$ concentrations to field site gas chromatograph value.

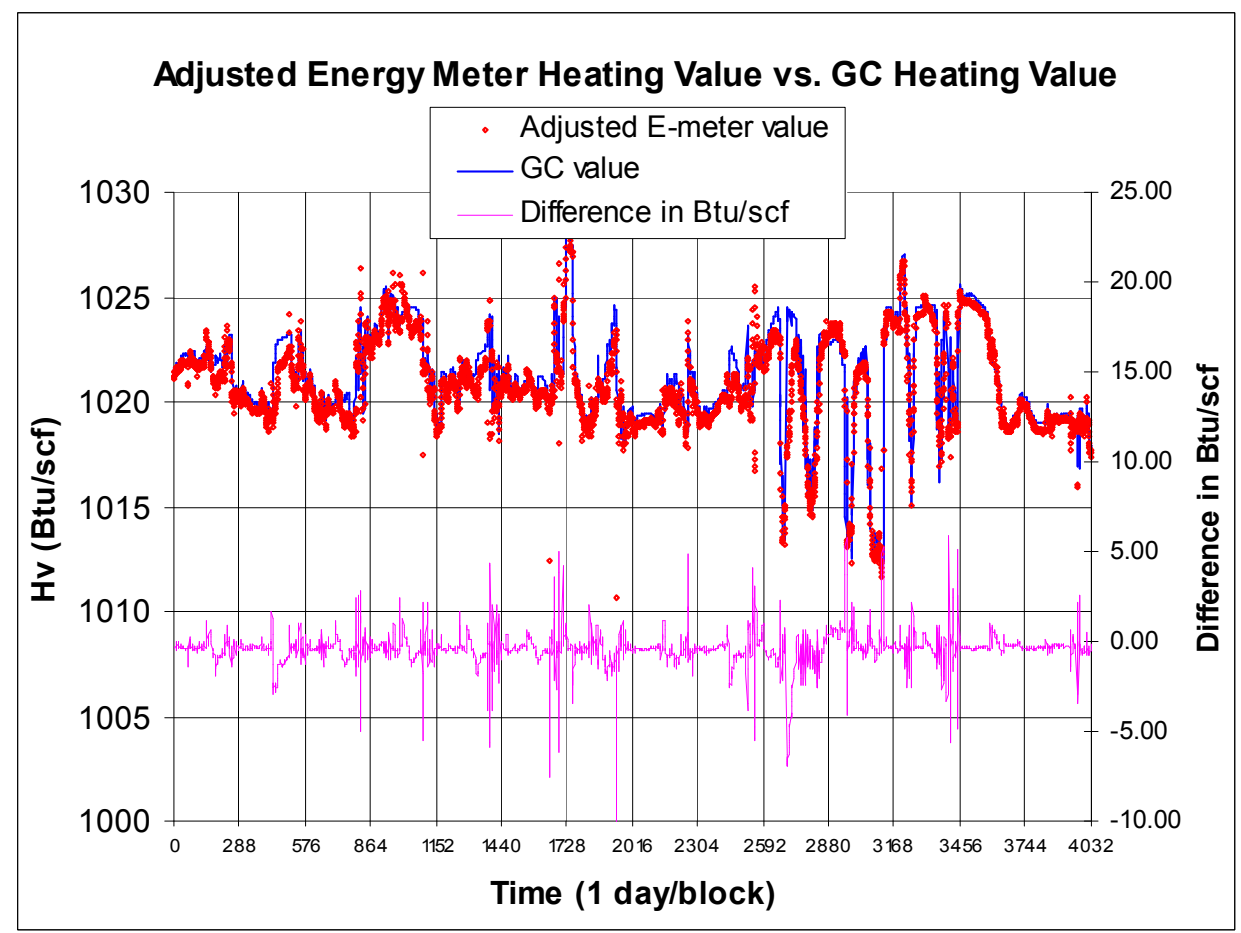

Figure 4.23. Adjusted energy meter heating value compared to field site gas chromatograph. 
Btu/scf. The spikes that occur in the middle of the two-week time period are explained by the lack of synchronization of the field data recorders. If flow in Line 2 increases or decreases very quickly in the line, temperature and speed of sound are often related to an earlier data point, resulting in the error spikes. Another potential cause of the erroneous data points is the reference itself - the field site gas chromatograph. If the gas chromatograph was not calibrated at regular intervals or was affected by varying pressure effects, erroneous reference values could have caused the algorithm disagreement. Figure 4.24 shows the data for the un-normalized total value over the entire two-week period, including periods of no flow. The un-normalized total is in the range of $100.5-101.5 \%$, higher than the desired total of $100 \%$, suggesting that GC recalibration may be in order.

Using the gathered field data, the current energy meter module configuration was partially simulated using the field site input data (including the constant nitrogen concentration), but with variable $\mathrm{CO}_{2}$ concentration, taken from the gas chromatograph data. This scenario simulates using the module in its present form, with carbon dioxide and speed-of-sound sensors, but without a nitrogen sensor. Figure 4.24 shows the effects that this scenario would have on the derived heating value. In computing this heating value, the nitrogen concentration was set at the first data point in the two-week data period, $1.337 \mathrm{~mol} \%$; a value representative of the two-week nitrogen concentration. In this simulation, had the module been installed at the field site, the algorithm would predict the actual gas heating value within a range of -5 to $+8 \mathrm{Btu} / \mathrm{scf}$ on the average, as shown in Figure 4.25.

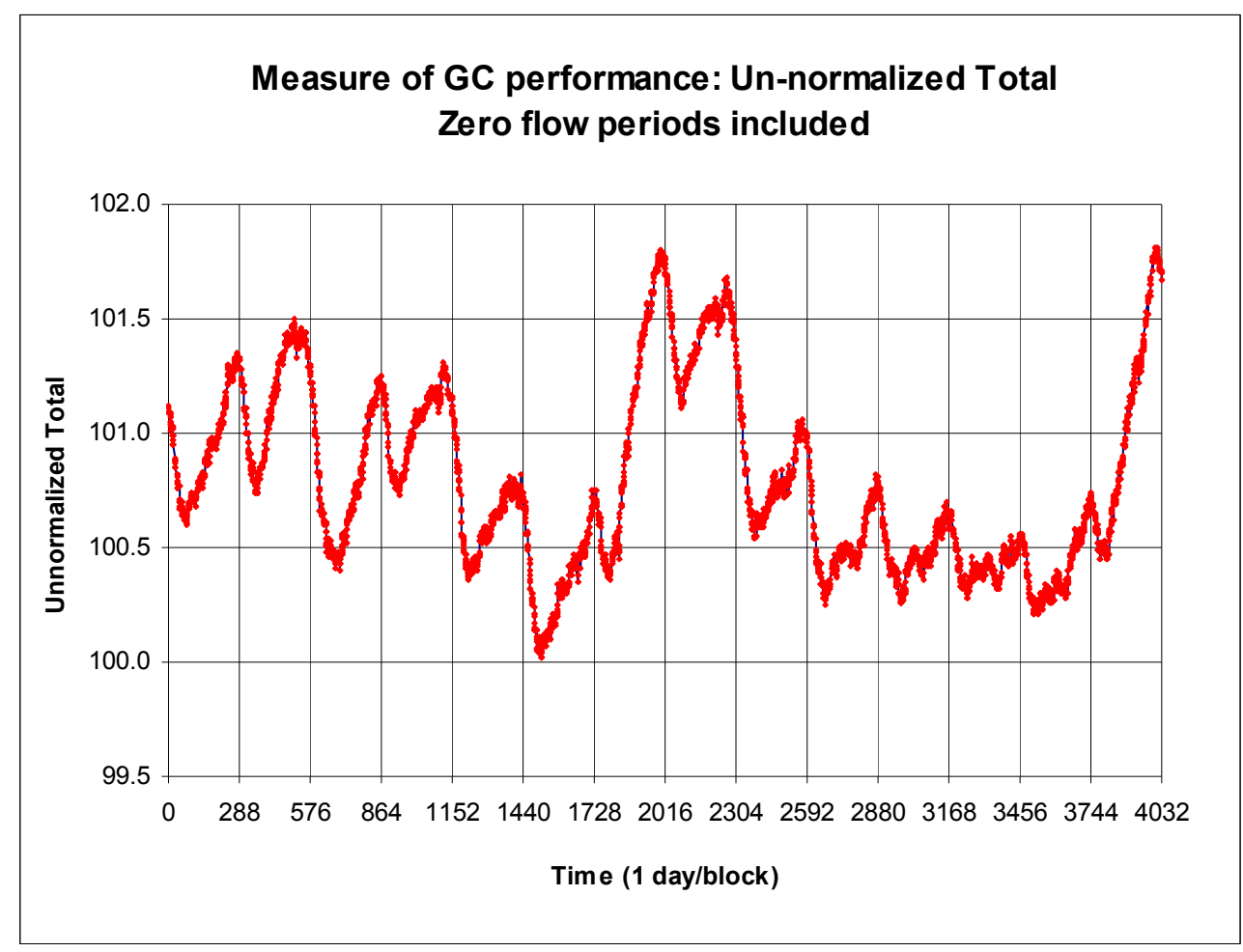

Figure 4.24. Field site gas chromatograph un-normalized total over two-week period. 


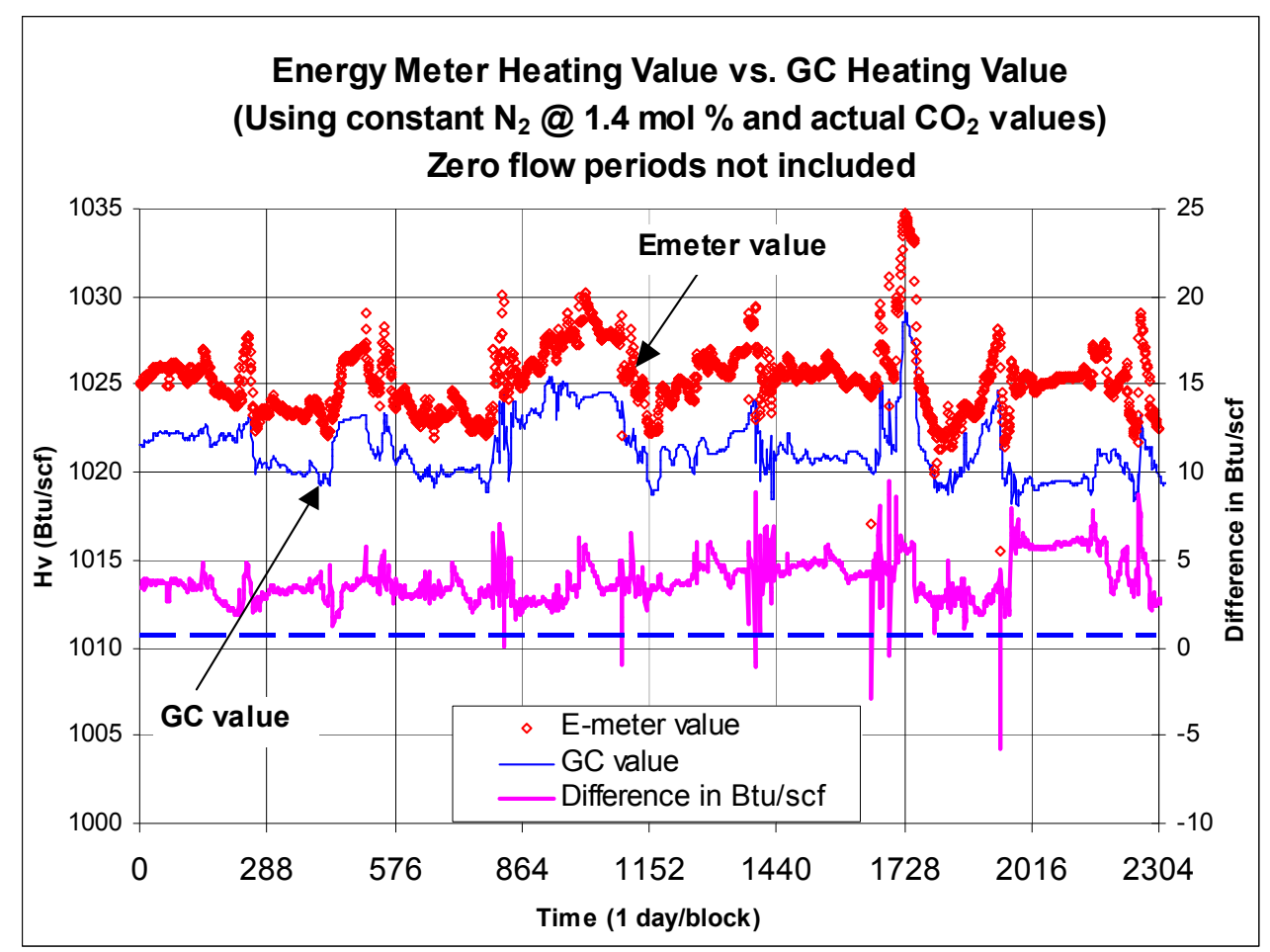

Figure 4.25. Energy meter heating value, compensated for $\mathrm{CO}_{2}$ only, compared to GC value. 
This page is intentionally blank. 


\subsection{Conclusions and Recommendations}

\subsection{Energy Meter Advances}

Project efforts during 2000-2001 resulted in significant advances in the investigation and development of the prototype natural gas energy meter system. Building upon the proof-ofconcept studies performed in 1999-2000, the following tasks were accomplished:

1. Algorithm studies were performed to evaluate the inferential gas energy method using a broader database of natural gas mixtures than that utilized in the earlier developments and to expand the sensitivity analysis of the method to develop more conclusive error bounds for speed-of-sound and carbon dioxide diluent measurements to match the accuracy attainable in the inferential algorithm. The total uncertainty in standard volumetric heating value is $\pm 2.4 \mathrm{Btu} / \mathrm{scf}$ (see Table 2.9), approximately $\pm 0.24 \%$ if $\mathrm{H}_{\mathrm{v}}=1009 \mathrm{Btu} / \mathrm{scf}$. This estimate of uncertainty is higher than the estimate of $\pm 1.85 \mathrm{Btu} / \mathrm{scf}$ determined by the single transmission-quality gas sensitivity analysis in 1999-2000. However, this new estimate includes the "worst case," where sensitivities, which are a function of gas composition, are higher than those expected for more typical natural gas mixtures. If the gas being measured is similar to the MRF distribution-quality gas used in the 1999-2000 analysis, the sensitivity coefficients will be smaller. The allowable measurement uncertainties will then lead to a smaller total uncertainty in $\mathrm{H}_{\mathrm{v}}$. The "worst case" coefficients in the present analysis are all derived from gas with a composition that includes higher amounts of ethane and heavier hydrocarbons than are found in typical transmission and distribution gases. Heating value uncertainties in typical transmissionquality natural gas will be smaller than this "worst-case" value. To reduce the worst-case uncertainty to $\pm 1.75 \mathrm{Btu} / \mathrm{scf}$, the error bounds on the speed-of-sound measurement were tightened from $\pm 1.5 \mathrm{ft} / \mathrm{s}$ to $\pm 0.8 \mathrm{ft} / \mathrm{s}$ and the error bounds on carbon dioxide and nitrogen diluent concentrations were reconfirmed to be within $\pm 0.05 \mathrm{~mol} \%$, respectively [Table 9 , Morrow et al. (2000)]. The new error bounds will also reduce the uncertainty associated with transmission-quality and distribution-quality gases from $\pm 1.85 \mathrm{Btu} / \mathrm{scf}$ to \pm 1.46 $\mathrm{Btu} / \mathrm{scf}$. These error bounds and energy determination uncertainties are now adopted as the performance goals for future energy meter system development.

2. Bench tests performed on the earlier speed-of-sound test chamber and time-of-flight measuring method revealed errors in accurate control and measurement of the gas temperature under varying ambient temperature conditions and in reliably extracting the ultrasonic time-of-flight difference from the two pulse reflections in the chamber. A new speed-of-sound test chamber design in which the chamber body served as a heat sink for the test gas, accurately tracking ambient temperature changes, resolved the gas temperature measurement and control problem. Timing accuracy and resolution errors in the difference between ultrasonic pulse travel times were corrected by using a new ultrasonic transducer operating at a higher center frequency and having a wider bandwidth than the previous transducer and a higher digital sampling rate in the data collection process. These hardware changes and the use of a two-reflector target made of low thermal expansion material brought the speed-of-sound measurement errors to well within the sensitivity tolerance required for determining the gas heating value to within $\pm 2.4 \mathrm{Btu} / \mathrm{scf}$. In the process of determining these modifications, other possible sources of 
error in the ultrasonic speed-of-sound measurement method were analyzed to establish their significance and to devise an effective calibration method for speed-of-sound measurements that could adequately compensate the method for all significant error effects. In addition to establishing a usable calibration technique, based on determining an 'effective' spacing dimension between the two pulse-reflecting targets, these studies established the optimum operating frequency range and bandwidth requirements for use in the next-generation energy meter system.

3. A commercial nondispersive infrared absorption $\mathrm{CO}_{2}$ sensor was tested and incorporated in the energy meter module for determining the concentration of diluent carbon dioxide in the natural gas. The sensing technology used in this device proved to be accurate and robust for measuring the $\mathrm{CO}_{2}$ content to within the bounds of $\pm 0.05 \mathrm{~mol} \%$ when mounted in a test chamber designed to accurately track changes in the energy meter module ambient temperature. A pressure rating limiting the sensor to operation at atmospheric conditions and nonlinearities in the response of this commercial sensor presently restrict the working range of $\mathrm{CO}_{2}$ measurements to an upper limit of about $3 \mathrm{~mol} \%$ in order to preserve the inferred gas heating value to within $\pm 2.4 \mathrm{Btu} / \mathrm{scf}$. However, with further development this sensing technology has an excellent potential for meeting the required natural gas sensing requirements, including potential operation at elevated pressures.

4. Progress on nitrogen sensing included identifying five potential technical approaches for determining the nitrogen diluent content in natural gas. Of these five methods, an inferential technique based on the absolute viscosity of the gas was found to offer the greatest promise for cost-effective use in the energy meter. This method, employing acoustical attenuation measurements caused by gas viscosity losses in sound transmission through a porous medium, has been developed in prototype form by Honeywell Corp. and can be made available on loan for future testing and experimental evaluation in natural gas. Preliminary investigation of the accuracy requirements for inferential determination of typical nitrogen concentrations in natural gas derived from experimental measurements of viscosity, speed of sound, and carbon dioxide concentration indicate the method to be potentially applicable provided that the viscosity sensor can resolve the gas mixture viscosity to within about $\pm 0.5 \%$. The other technical approaches, although technically sound, are either still too early in development to conclusively state their sensing accuracy or otherwise much too expensive for adaptation to the present energy meter application.

5. Laboratory tests of the improved energy meter module were performed on the MRF highpressure flow loop to evaluate the performance of the module using normal transmissionquality gas under different pipeline temperature and flow conditions and a reasonable range of summer outdoor ambient conditions. The results of these tests, performed over a four-day time period using a known gas composition, yielded gas heating values that were well within the $\pm 2.4 \mathrm{Btu} / \mathrm{scf}$ worst-case error bounds for the inferential method. When these measurements were converted to energy flow rate, the energy flow rate errors were less than $2 \%$ when using ultrasonic flow meter accuracies. Similar laboratory tests were performed on the MRF low-pressure flow loop using gas mixtures having carbon dioxide diluent content ranging from about $1.2 \mathrm{~mol} \%$ to $9 \mathrm{~mol} \%$ by doping the loop with supplemental $\mathrm{CO}_{2}$. Tests on the normal gas (approx. $1.2 \mathrm{~mol} \%$ ) confirmed the energy meter performance observed in the previous high-pressure loop tests. Tests on a gas having $3 \mathrm{~mol} \% \mathrm{CO}_{2}$ concentration also confirmed the energy meter performance 
accuracy. Tests at $\mathrm{CO}_{2}$ concentrations of $6 \mathrm{~mol} \%$ and $9 \mathrm{~mol} \%$ revealed significant offsets and nonlinear effects in the sensor response and a noticeable amount of added scatter in the derived gas heating value for gas mixtures having more than $3 \mathrm{~mol} \% \mathrm{CO}_{2}$ concentration. However, when the offset bias errors were removed, the residual errors at the higher $\mathrm{CO}_{2}$ concentrations were less than $\pm 2.4 \mathrm{Btu} / \mathrm{scf}$ in all cases.

6. A field test was conducted at a gas-fired power plant for the purpose of evaluating the inferential algorithm and the interfacing and data collection processes needed for adapting the energy meter module to practical field site conditions. Data were collected over a continuous two-week time period to allow two evaluation scenarios: (1) representing installations in which the inferential algorithm alone is used in combination with only fixed diluent gas concentrations (obtained on the first day of testing) plus speed-of-sound data obtained from locally installed ultrasonic flow meters in the monitored gas lines; and (2) representing an approximate simulation of the present energy meter module whereby updated values of $\mathrm{CO}_{2}$ concentration are obtained at frequent intervals from the locally installed gas chromatograph but nitrogen concentration is held at its fixed nominal value plus speed-of-sound data provided by a local ultrasonic flow meter. Practical factors encountered in the data collection process included the fact that the update timing in the local flow computers and the time sampling of inputs to the algorithm data logger could not be synchronized and normal operation of the power plant introduced diurnal changes in gas demand flow that introduced transient effects in the recorded data and associated inferential gas energy determinations. These effects were minimized in analyzing the data by analyzing only those data recorded after the transient disturbances died away. The gas heating value derived in the first evaluation scenario (constant $\mathrm{CO}_{2}$ and $\mathrm{N}_{2}$ concentrations) was within $\pm 10 \mathrm{Btu} / \mathrm{scf}$ at all times with respect to the value derived by gas chromatograph analysis. When the field data were processed again using the monitored values of $\mathrm{CO}_{2}$ concentration, the agreement with the gas chromatograph value was within -5 to $+8 \mathrm{Btu} / \mathrm{scf}$.

\subsection{Conclusions}

The laboratory flow loop tests of the inferential heating value algorithm and the current prototype energy meter module measuring speed of sound and carbon dioxide concentration (nitrogen concentration fixed at GC value) have confirmed the validity and accurate functions of the energy meter sensors and the overall system methodology. For the samples of normal transmission-quality natural gas tested, the energy meter sensors provided inputs to the algorithm that were within the tolerances needed to satisfy inferred gas heating values to within less than the worst-case tolerance of $\pm 2.4 \mathrm{Btu} / \mathrm{scf}$ relative to the values derived from the gas chromatograph readings. The field tests representing the use of the algorithm alone, relying on fixed values of diluent gas concentrations and speed-of-sound data from an ultrasonic flow meter and edited to remove excessive transient flow variations, were found to have error bounds less than $\pm 5 \mathrm{Btu} / \mathrm{scf}$, on average during a two-week test period. Although the transient effects were removed from the analyzed data, the wider error bounds in the field test results were indirectly affected by attendant variations occurring in the gas chromatograph readings used as the heating value reference. Overall, the essential results of the field and laboratory tests indicate that the current prototype energy meter performance is within the inherent accuracy limits of the inferential algorithm, particularly for field site conditions where no-flow transient conditions are absent. 
This conclusion now suggests that the operating characteristics of the speed-of-sound sensor and the carbon dioxide sensor may be used as the basis for specifying and optimizing these sensors for final development as industry-qualified devices for use in natural gas field environments.

Progress toward obtaining a practical method for determining the nitrogen concentration in natural gas during 2000-2001 identified four potential methods and, on evaluation, led to rejecting three of the methods as either being too uncertain in their accuracy or excessive in cost for use in the planned energy meter. One prospective candidate method, based on inferential correlation of measured gas-mixture absolute viscosity with molecular nitrogen concentration, was identified for possible near-future testing and evaluation. The gas viscosity sensor identified for use in this method is under development by Honeywell Corp. and will be tested by SwRI in 2002.

\subsection{Recommendations}

Recommendations for continued development of the energy meter module during 20012002 place emphasis on firmly specifying and upgrading the prototype sensors and associated electronics system, further evaluating the refined module, and identifying and evaluating appropriate techniques for accurately determining nitrogen content in natural gas. Work tasks related to these recommendations include:

1. Design and fabricate a compact electronics system for implementing the ultrasonic speedof-sound measurements and signal processing. This system will replace the general purpose portable computer and other laboratory equipment now used with the module, resulting in a revised system that is optimized in operation and self-contained in a single module.

2. Specify and procure an improved ultrasonic transducer aimed at achieving the optimized performance capabilities established through the 2000-2001 transducer studies and evaluations.

3. Conduct further evaluation tests of the current energy meter module using a wide range of diluent concentrations in natural gas mixtures with the module exposed to environmental temperature extremes. These tests will establish the robustness of the energy meter sensors and the boundaries of gas mixture compositions for accurate gas heating value determinations using the present technology.

4. Conduct extended performance tests of the carbon dioxide sensor to determine its potential use at elevated pressures. A first goal will be to adapt the sensor to operate at the same pressure used in the speed-of-sound test chamber to eliminate the need for a separate $\mathrm{CO}_{2}$ test chamber and associated separate gas temperature and pressure sensors. An extended goal will be to explore the feasibility of adapting the $\mathrm{CO}_{2}$ IR sensing technique for operating directly at gas transmission and distribution pipeline pressures.

5. Continue to identify and evaluate promising nitrogen sensing techniques for use in the energy meter module. The Honeywell gas viscosity sensor is one such technique that offers a potentially inexpensive and accurate capability for this application. Additionally, since no direct methods for sensing molecular nitrogen have been identified, other methods based either on indirect or inferential determination of nitrogen will be postulated and evaluated for practical feasibility.

6. Deploy the upgraded prototype energy meter module in a minimum of two field tests designed to evaluate both the sensing technology and the adaptability of the energy meter system to practical field operating conditions. 


\subsection{References}

American Gas Association [1992]. Compressibility Factors of Natural Gas and Other Related Hydrocarbon Gases. AGA Transmission Measurement Committee Report No. 8, American Gas Association, Arlington, Virginia, USA.

American Gas Association [1994]. Compressibility Factors of Natural Gas and Other Related Hydrocarbon Gases. AGA Transmission Measurement Committee Report No. 8, American Gas Association, Arlington, Virginia, USA.

Behring II, K. A., Kelner, E., Minachi, A., Sparks, C. R., Morrow, T. B., and Svedeman, S. J. [1999]. A Technology Assessment and Feasibility Evaluation of Natural Gas Energy Flow Measurement Alternatives. Final Report, Tasks A and B, DOE Cooperative Agreement No. DE-FC21-96MC33033, Southwest Research Institute, San Antonio, Texas, USA.

Bonné, U., and Kubisiak, D. [2001]. “Actuation-Based, Natural Gas Microsensors,” Proceedings of the GTI 2001 Natural Gas Quality, Energy Measurement, Metering, and Utilization Conference, March 5-6.

Bruno, T. J., and Haynes, W. M. [1993]. Thermophysical Properties of Fluids for the Gas Industry. Final Report, February 1988-August 1993, Gas Research Institute, Chicago, Illinois, USA.

Callahan, T. J. [2001]. Internal correspondence, Southwest Research Institute, San Antonio, Texas, USA.

Dain, Y., and Lueptow, R. M. (2001a). "Acoustic Attenuation in Three-Component Gas Mixtures: Theory,” J. Acoust. Soc. Am., Vol. 109, No. 5, pp. 1955-1964.

Dain, Y., and Lueptow, R. M. (2001b). "Acoustic Attenuation in a Three-Gas Mixture: Result," J. Acoust. Soc. Am., Vol. 110, No. 6, pp. 2974-2979.

Dayton, T., Beyerlein, S., Savidge, J. L., and Goodwin, A. R. H. [1999]. Assigned to Gas Research Institute, "Apparatus and Method for Determining Thermophysical Properties Using an Isochoric Approach,” U.S. Patent No. 5,932,793, August 3, 1999; assigned to GRI.

De Los Santos, A. [2001]. "Nuclear magnetic resonance as a component to a prototype natural gas analysis custody transfer instrument module," memorandum to E. Kelner and D. George, Southwest Research Institute, October 9.

Diller, D. E., and Magee, J. W. [2000]. Thermophysical Properties of Mixtures of Natural Gas Components: A Bibliography of Experimental Data. Report NISTIR 5100, National Institute of Standards and Technology, Boulder, Colorado, USA.

Gas Processors Association [1994]. Table of Physical Constants of Paraffin Hydrocarbons and Other Components of Natural Gas. GPA Standard 2145-94, Gas Processors Association, Tulsa, Oklahoma, USA.

Gas Processors Association [2000]. Table of Physical Constants for Hydrocarbons and Other Compounds of Interest to the Natural Gas Industry. GPA Standard 2145-00, Revision 2, Gas Processors Association, Tulsa, Oklahoma, USA. 
Gas Research Institute [1998]. GRI's Gas Resource Database, Unconventional Natural Gas and Gas Composition Databases. CD-ROM Report GRI-98/0364.3, Gas Research Institute, Chicago, Illinois, USA.

Herzfeld, K. F. and Litovitz, T. A. (1959). Absorption and Dispersion of Ultrasonic Waves, Academic Press, New York, NY, USA.

Holste, J. C., and Hall, K. R. [1993]. Thermodynamic Properties of Natural Gas Mixtures. Final Report, February 1987-September 1992, Gas Research Institute, GRI-93/0006, Chicago, Illinois, USA.

Interstate Natural Gas Association of America [1995]. Grid Integration Project, Gas Quality Task Force. Final Report. Washington, D.C.

King, J. D., Rollwitz, W. L., and Nicholls, C. I. [1988]. Nuclear Magnetic Resonance for Measuring Gas Energy Content. Final Report, September 1986-April 1988, for Gas Research Institute, Chicago, Illinois, USA.

King, J. D., and Nicholls, C. I. [1990]. "Gas Properties Measured by Magnetic Resonance," Proceedings of the IGT Gas Quality Measurement Workshop, July, Chicago, Illinois, USA.

Koskinen, Y., Lehto, A., Tammela, S., Blomberg, M., Orpana, M. and Torkkeli, A. [1997]. "Single Channel Gas Concentration Measurement Method and Apparatus Using a ShortResonator Fabry-Perot Interferometer," U.S. Patent No. 5,646,729; July 8, 1997; Assigned to Vaisala Oy, Helsinki, Finland.

Lomic, Inc. [1997]. SonicWare ${ }^{\circledR}$, Version 2.0; Lomic, Inc., State College, PA, USA.

Minachi, A., Margetan, F. J., and Thompson, R. B. [1997]. "Reconstruction of a Piston Transducer Beam Using Multi-Gaussian Beams (MGB) and Its Application," Review of Progress in Quantitative Nondestructive Evaluation, Vol. 17A.

Morrow, T. B., Kelner, E., and Minachi, A. [2000]. Development of a Low Cost Inferential Natural Gas Energy Flow Rate Prototype Retrofit Module," Final Report, DOE cooperative Agreement No. DE-FC21-96MC33033, Sept. 2000. Southwest Research Institute, San Antonio, Texas, USA.

Nicholls, C. I., and King, J. D. [1989]. "On Line Measurement of Natural Gas Calorific Values by Nuclear Magnetic Resonance," Proceedings of the IGT Gas Measurement Workshop, June 1989, Chicago, Illinois, USA.

Savidge, J. L. [1989]. FORTRAN program SUPERZ, Natural Gas Thermodynamic Properties Package (A.G.A. No. 8), March, SCS, Inc.

Starling, K. E., Fitz, C. W., Chen, Y. C., Rondon, E., Jacobsen, R. T., Beyerlein, S. W., Clarke, W. P., and Lemmon, E. [1991]. GRI High Accuracy Natural Gas Equation of State for Gas Measurement Applications. Annual Report, May 1990-April 1991, for Gas Research Institute, Chicago, Illinois, USA.

Van Wylen, G. J., Sonntag, R. E., and Borgnakke, C. [1994]. Fundamentals of Classical Thermodynamics. Wiley and Sons, New York, NY, USA.

Watson, J. T. R. [1998]. A Report on the Density of Rich Natural Gases, a Joint Industrial Project. National Engineering Laboratory, East Kilbride, Glasgow, UK. 
Westbrook, S. R. [1993]. "Army Use of Near-Infrared Spectroscopy to Estimate Selected Properties of Compression Ignition Fuels," SAE International Congress and Exposition, March $1-5$, Detroit, Michigan, USA.

Westbrook, S. R. [1994]. "The Feasibility of Using a Near-Infrared Spectrometer to Estimate Selected Properties of Natural Gas," SAE International Congress and Exposition, February 28March 3, Detroit, Michigan, USA.

Younglove, B. A., Frederick, N. V., and McCarty, R. D. [1993]. Speed of Sound Data and Related Models for Mixtures of Natural Gas Constituents. Report GRI-92/0211, Gas Research Institute, Chicago, Illinois, USA.

Zhang, W., and Schouten, J. A. [1992]. The Sound Velocity of a Mixture of $\mathrm{CH}_{4}$ and $\mathrm{N}_{2}$ from 2 kbar to 8 kbar and from $173 \mathrm{~K}$ to 298 K. Physica A, Vol. 189, pp. 493-502. 
This page is intentionally blank. 


\section{Appendix: Bench Tests of Speed-of-Sound Transducers}

\section{A.1 Introduction}

Bench tests were performed to evaluate the accuracy of the time-of-flight method for measuring the speed of sound in several known gas mixtures. The experimental test results were compared with speed-of-sound values calculated using the AGA-8 equation of state as implemented in the Lomic, Inc. (1997) SonicWare ${ }^{\circledR}$ software package. Five ultrasonic transducers capable of air-coupled operation in the frequency range of $200-1000 \mathrm{kHz}$ were tested:
(1) $\quad 200 \mathrm{kHz} \quad$ (Siemens DGT speed-of-sound sensor; 0.5-in. dia.)
(2) $\quad 220 \mathrm{kHz} \quad$ (Massa Products Model E-188/220; 0.5-in. dia.)
(3) $\quad 500 \mathrm{kHz}$ (Ultran Model NCT-105; 1.0-in. dia.)
(4) $\quad 1000 \mathrm{kHz}$ (Ultran Model NCT-510; 0.57-in. dia.)
(5) $\quad 279 \mathrm{kHz} \quad$ (Etalon Model CIA-3525-SB; 1.0-in. dia.)

The gases used in the tests were:

(1) Pure Nitrogen (Ref: Cyl \#EE2220)

(2) Ultra-Pure Methane (Ref: Cyl \#16732 )

(3) Natural Gas \#1 (methane 75.103\%; ethane 12.500\%; propane 5.900\%; isobutane $1.010 \%$; n-butane $1.610 \%$; isopentane $0.181 \%$; n-pentane $0.240 \%$; n-hexane $0.146 \%$; n-heptane $0.067 \%$; n-octane $0.049 \%$; n-nonane $0.012 \%$; n-decane $0.006 \%$; carbon dioxide $0.406 \%$; nitrogen $2.770 \%$ ) (Ref: Cyl \#35016)

(4) Natural Gas \#2 (methane 95.261\%; ethane 2.105\%; propane 0.505\%; isobutane $0.070 \%$; n-butane $0.122 \%$; isopentane $0.037 \%$; n-pentane $0.029 \%$; n-hexane $0.023 \%$; n-heptane $0.020 \%$; n-octane $0.011 \%$; n-nonane $0.001 \%$; carbon dioxide $1.116 \%$; nitrogen $0.700 \%$ ) (Ref: MRF flow loop sample collected 4/19/01)

(5) Natural Gas \#3 (methane 94.477\%; ethane 2.510\%; propane $0.499 \%$; isobutane $0.100 \%$; n-butane $0.100 \%$; isopentane $0.050 \%$; n-pentane $0.100 \%$; n-hexane $0.020 \%$; n-heptane $0.020 \%$; n-octane $0.010 \%$; carbon dioxide $1.000 \%$; nitrogen $1.010 \%$; argon 0.104 \%) (Ref: Cyl \#XL002486)

\section{A.2 Experimental Test Parameters and Method}

Gas Temperature: In anticipation of a large number of experimental test runs, the bench tests were elected to be performed at laboratory room temperature, which was well regulated within the range of 73-75EF. The speed-of-sound test chamber components and the gas supply cylinders were equilibrated to room temperature throughout the test period, and the room air temperature was measured at frequent intervals (typically about every 8 minutes) during the data acquisition periods, using an RTD sensor having an accuracy of $\pm 0.1 \mathrm{EF}$. The test chamber and air temperature sensor were located away from any room ventilation air circulation paths.

Gas Pressure: The ultrasonic transducers were mounted in the prototype speed-of-sound test chamber with pressure exposure only on the active face. Therefore, the maximum test pressure was restricted to 100 psia in order to avoid possible damage to the transducer devices. 
Speed-of-sound measurements were recorded at pressure increments of approximately 10 psi over the range from local barometric pressure (typically 14.5 psia) up to 100 psia. Pressure was measured using a Parascientific Model 740 (0-150 psia) digital display pressure gauge having an accuracy of $\pm 0.01 \mathrm{psi}$. For some of the gases, the pressure available in the gas supply cylinders was not sufficient to allow measurements above about $75 \mathrm{psia}$ and, therefore, not all test runs covered the pressure range up to 100 psia.

Prototype Test Chamber: Ultrasonic pulse time-of-flight measurements were performed using a single transmit-receive transducer mounted at one end of a cylindrical aluminum test chamber fitted with a dual-target reflector plug mounted in the opposite end. The two half-circle faces of the reflector plug were separated by a fixed distance of approximately an inch (measured to within an accuracy of $\pm 0.2 \times 10^{-3}$ in.) to provide two reflected ultrasonic pulses having a common time origin and an accurately known propagation path length difference. Several interchangeable dual-target reflector plugs having different insertion distances into the cylindrical chamber were available to provide different two-way sound propagation path lengths. The axial separation of the reflecting faces on all of these insert plugs was approximately an inch. The sound reflection path length was changeable in increments of approximately an inch to provide path distances between the nearest reflector face and the transducer face ranging from 1.25 to $4.25 \mathrm{in}$. The inside diameter of the test chamber was $1.50 \mathrm{in}$. Three drilled and tapped holes in the wall of the test chamber provided gas inlet and outlet transfer and a connection for pressure measurement.

Ultrasonic Pulse Excitation, Detection, and Recording: The ultrasonic transducer under test was excited by a gated sine wave electrical signal adjusted in frequency and pulse width to provide a compact and well-defined sinusoidal pulse wavelet as observed in the reflected waveforms. The excitation signals were generated by a portable BSI FieldGo industrial personal computer equipped with a Matek Model TB-1000 Toneburst Pulser/Receiver (300V p-p; gated sine wave; $50 \mathrm{kHz}-20 \mathrm{MHz}$ ) and software capabilities for generating gated sine wave signals at frequencies selectable in $50-\mathrm{kHz}$ increments with pulse widths adjustable in $1-\mu$ s increments. The pulse repetition rate used throughout the various tests was $1,000 \mathrm{pulses} / \mathrm{s}$. The repetitive received ultrasonic signals, containing two time-displaced reflections, were time-averaged for either 64 or 128 traces to improve their signal-to-noise ratio and displayed by a Tektronix Model TDS-3012 digital oscilloscope. The displayed waveforms were also digitally recorded on a 3.5-in. magnetic diskette by the TDS-3012 oscilloscope using a digital time sampling rate of 25 $10^{6} \mathrm{samples} / \mathrm{s}$, corresponding to a time-of-flight resolution of 40 nanoseconds to resolve the derived speed of sound to within $\pm 0.35 \mathrm{ft} / \mathrm{s}$ to $\pm 0.45 \mathrm{ft} / \mathrm{s}$, depending on the gas mixture under test.

Time-of-Flight Data Analysis: The recorded waveforms were processed by numerical analysis to yield the cross-correlation function and, in particular, an accurate measure of the time difference between the two reflected signals in each trace. The correlation time delay associated with the highest peak in the correlation function was selected automatically by the analysis software to yield the differential time-of-flight between the two reflector faces. The speed of sound was then calculated using the relation

$$
v_{g}=\left(\frac{10^{6}}{6}\right)\left(\frac{\Delta D}{\Delta t}\right)
$$


where

$$
\begin{aligned}
\Delta D & =\text { distance between reflector faces (in.) } \\
\Delta t & =\text { correlation time lag }(\mu \mathrm{s})
\end{aligned}
$$

\section{A.3 Calculated Speed of Sound in Test Gas Mixtures}

The speed of sound in the five gases used in the tests was predicted using the AGA-8 equation of state implemented in the SonicWare ${ }^{\circledR}$ software package. Table A.1 lists the predicted values for three temperatures and for pressures ranging from 15 psia to 100 psia. Tabulated with a resolution of $0.1 \mathrm{ft} / \mathrm{s}$, these values have an accuracy and resolution of $\pm 0.004 \%$, or better. Figure A.1 shows all of the data in Table A.1 to illustrate the nearly uniform pressure and temperature dependence of the speed of sound in the five test gases for the temperature and pressure ranges used. To clearly show these relative characteristics, the speed-of-sound values in Figure A.1 are scaled to match the value for Ultra-Pure Methane at 14.73 psia.

For the narrow range of temperatures for which the tests were conducted, the calculated speed of sound may be accurately adjusted to match the average temperature of the ultrasonic test chamber temperature by applying an ideal gas law absolute temperature scaling factor. For example, for speed-of-sound values calculated at 74EF, the temperature scaling factor is

$$
S_{T}=\sqrt{\frac{491.67 R+T_{a v g}^{\circ} F}{491.67 R+74^{\circ} F}}
$$

where $\mathrm{T}_{\mathrm{avg}}=$ average temperature of the test chamber $(\mathrm{EF})$.

\section{A.4 Bench Test Procedure}

The speed-of-sound tests were performed at near-constant temperature without gas flow through the ultrasonic test chamber. Valves at the inlet and outlet ports of the test chamber allowed the chamber to be charged with one filling of the test gas at a pressure somewhat higher than the highest pressure of interest (between 75 psia and 100 psia) and then bled down in approximate 10-psi steps to obtain successive measurements of speed of sound versus pressure. The chamber was first purged by cycling the chamber pressure from the highest pressure to atmospheric pressure 20 times to ensure that no significant trace of the gas from the previous test was present. After setting the pressure at each test value, a time period of approximately 6-7 minutes was allowed for the gas to equilibrate to the temperature of the test chamber. Therefore, the bench test data were acquired at a rate of one test record every 8 minutes. The air temperature in the immediate vicinity of the test chamber was recorded at each test reading and was noted to vary only about $\pm 1 \mathrm{EF}$ during the approximate 50-60 minute test period for each gas. The amplitude of the excitation signal applied to the ultrasonic transducer was held fixed throughout each test run; however, since the transducer coupling into the gas varied with the gas pressure, affecting the amplitude of the received signal, the received signal amplitude was adjusted for optimum signal-to-noise ratio and oscilloscope display at each pressure setting before being digitally recorded. 
Table A.1. Calculated speed of sound for five test gases (SonicWare ${ }^{\circledR}$ ).

\begin{tabular}{|c|c|c|c|c|c|c|c|c|c|c|c|c|c|c|c|}
\hline \multirow[b]{2}{*}{$\begin{array}{l}\text { Pressure } \\
\text { (psia) }\end{array}$} & \multicolumn{3}{|c|}{ Pure Nitrogen } & \multicolumn{3}{|c|}{ Ultra-Pure Methane } & \multicolumn{3}{|c|}{ Natural Gas \#1 } & \multicolumn{3}{|c|}{ Natural Gas \#2 } & \multicolumn{3}{|c|}{ Natural Gas \#3 } \\
\hline & $73^{\circ} \mathrm{F}$ & $74^{\circ} \mathrm{F}$ & $75^{\circ} \mathrm{F}$ & $73^{\circ} \mathrm{F}$ & $74^{\circ} \mathrm{F}$ & $75^{\circ} \mathrm{F}$ & $73^{\circ} \mathrm{F}$ & $74^{\circ} \mathrm{F}$ & $75^{\circ} \mathrm{F}$ & $73^{\circ} \mathrm{F}$ & $74^{\circ} \mathrm{F}$ & $75^{\circ} \mathrm{F}$ & $73^{\circ} \mathrm{F}$ & $74^{\circ} \mathrm{F}$ & $75^{\circ} \mathrm{F}$ \\
\hline 14.73 & $1150 / 8$ & 1151.9 & 1152.9 & 1466.3 & 1467.6 & 1468.8 & 1238.0 & 1239.0 & 1240.1 & 1418.2 & 1419.5 & 1420.7 & 1412.4 & 1413.6 & 1414.8 \\
\hline 19.73 & 1150.9 & 1152.0 & 1153.1 & 1465.9 & 1467.2 & 1468.5 & 1237.2 & 1238.2 & 1239.3 & 1417.8 & 1419.0 & 1420.2 & 1411.9 & 1413.2 & 1414.4 \\
\hline 24.73 & 1151.1 & 1152.2 & 1153.3 & 1465.6 & 1466.8 & 1468.1 & 1236.4 & 1237.4 & 1238.5 & 1417.4 & 1418.6 & 1419.8 & 1411.5 & 1412.7 & 1413.9 \\
\hline 29.73 & 1151.3 & 1152.4 & 1153.4 & 1465.2 & 1466.5 & 1467.7 & 1235.6 & 1236.7 & 1237.7 & 1417.0 & 1418.2 & 1419.4 & 1411.1 & 1412.3 & 1413.5 \\
\hline 34.73 & 1151.4 & 1152.5 & 1153.6 & 1464.8 & 1466.1 & 1467.4 & 1234.8 & 1235.9 & 1236.9 & 1416.5 & 1417.8 & 1419.0 & 1410.7 & 1411.9 & 1413.1 \\
\hline 39.73 & 1151.6 & 1152.7 & 1153.8 & 1464.4 & 1465.7 & 1467.0 & 1234.0 & 1235.1 & 1236.2 & 1416.1 & 1417.3 & 1418.6 & 1410.2 & 1411.5 & 1412.7 \\
\hline 44.73 & 1151.8 & 1152.9 & 1153.9 & 1464.1 & 1465.3 & 1466.6 & 1233.2 & 1234.3 & 1235.4 & 1415.7 & 1416.9 & 1418.2 & 1409.8 & 1411.0 & 1412.3 \\
\hline 49.73 & 1151.9 & 1153.0 & 1154.1 & 1463.7 & 1465.0 & 1466.2 & 1232.4 & 1233.5 & 1234.6 & 1415.3 & 1416.5 & 1417.7 & 1409.4 & 1410.6 & 1411.8 \\
\hline 54.73 & 1152.1 & 1153.2 & 1154.3 & 1463.3 & 1464.6 & 1465.9 & 1231.7 & 1232.7 & 1233.8 & 1414.8 & 1416.1 & 1417.3 & 1409.0 & 1410.2 & 1411.4 \\
\hline 59.73 & 1152.3 & 1153.4 & 1154.5 & 1462.9 & 1464.2 & 1465.5 & 1230.9 & 1232.0 & 1233.1 & 1414.4 & 1415.7 & 1416.9 & 1408.5 & 1409.8 & 1411.0 \\
\hline 64.73 & 1152.5 & 1153.5 & 1154.6 & 1462.6 & 1463.9 & 1465.2 & 1230.1 & 1231.2 & 1232.3 & 1414.0 & 1415.3 & 1416.5 & 1408.1 & 1409.4 & 1410.6 \\
\hline 69.73 & 1152.6 & 1153.7 & 1154.8 & 1462.2 & 1463.5 & 1464.8 & 1229.3 & 1230.4 & 1231.5 & 1413.6 & 1414.8 & 1416.1 & 1407.7 & 1408.9 & 1410.2 \\
\hline 74.73 & 1152.8 & 1153.9 & 1155.0 & 1461.8 & 1463.1 & 1464.4 & 1228.5 & 1229.6 & 1230.7 & 1413.2 & 1414.4 & 1415.7 & 1407.3 & 1408.5 & 1409.8 \\
\hline 79.73 & 1153.0 & 1154.1 & 1155.2 & 1461.5 & 1462.8 & 1464.1 & 1227.7 & 1228.9 & 1230.0 & 1412.8 & 1414.0 & 1415.3 & 1406.9 & 1408.1 & 1409.4 \\
\hline 84.73 & 1153.2 & 1154.2 & 1155.3 & 1461.1 & 1462.4 & 1463.7 & 1227.0 & 1228.1 & 1229.2 & 1412.4 & 1413.6 & 1414.9 & 1406.4 & 1407.7 & 1408.9 \\
\hline 89.73 & 1153.3 & 1154.4 & 1155.5 & 1460.7 & 1462.0 & 1463.4 & 1226.2 & 1227.3 & 1228.4 & 1411.9 & 1413.2 & 1414.5 & 1406.0 & 1407.3 & 1408.5 \\
\hline 94.73 & 1153.5 & 1154.6 & 1155.7 & 1460.4 & 1461.7 & 1463.0 & 1225.4 & 1226.5 & 1227.7 & 1411.5 & 1412.8 & 1414.1 & 1405.6 & 1406.9 & 1408.1 \\
\hline 99.73 & 1153.7 & 1154.8 & 1155.9 & 1460.0 & 1461.3 & 1462.6 & 1224.6 & 1225.8 & 1226.9 & 1411.1 & 1412.4 & 1413.7 & 1405.2 & 1406.5 & 1407.7 \\
\hline 104.73 & 1153.9 & 1155.0 & 1156.1 & 1459.7 & 1461.0 & 1462.3 & 1223.8 & 1225.0 & 1226.1 & 1410.7 & 1412.0 & 1413.3 & 1404.8 & 1406.1 & 1407.3 \\
\hline 109.73 & 1154.1 & 1155.1 & 1156.2 & 1459.3 & 1460.6 & 1461.9 & 1223.1 & 1224.2 & 1225.4 & 1410.3 & 1411.6 & 1412.9 & 1404.4 & 1405.7 & 1406.9 \\
\hline 114.73 & 1154.2 & 1155.3 & 1156.4 & 1458.9 & 1460.3 & 1461.6 & 1222.3 & 1223.4 & 1224.6 & 1409.9 & 1411.2 & 1412.5 & 1404.0 & 1405.2 & 1406.5 \\
\hline
\end{tabular}




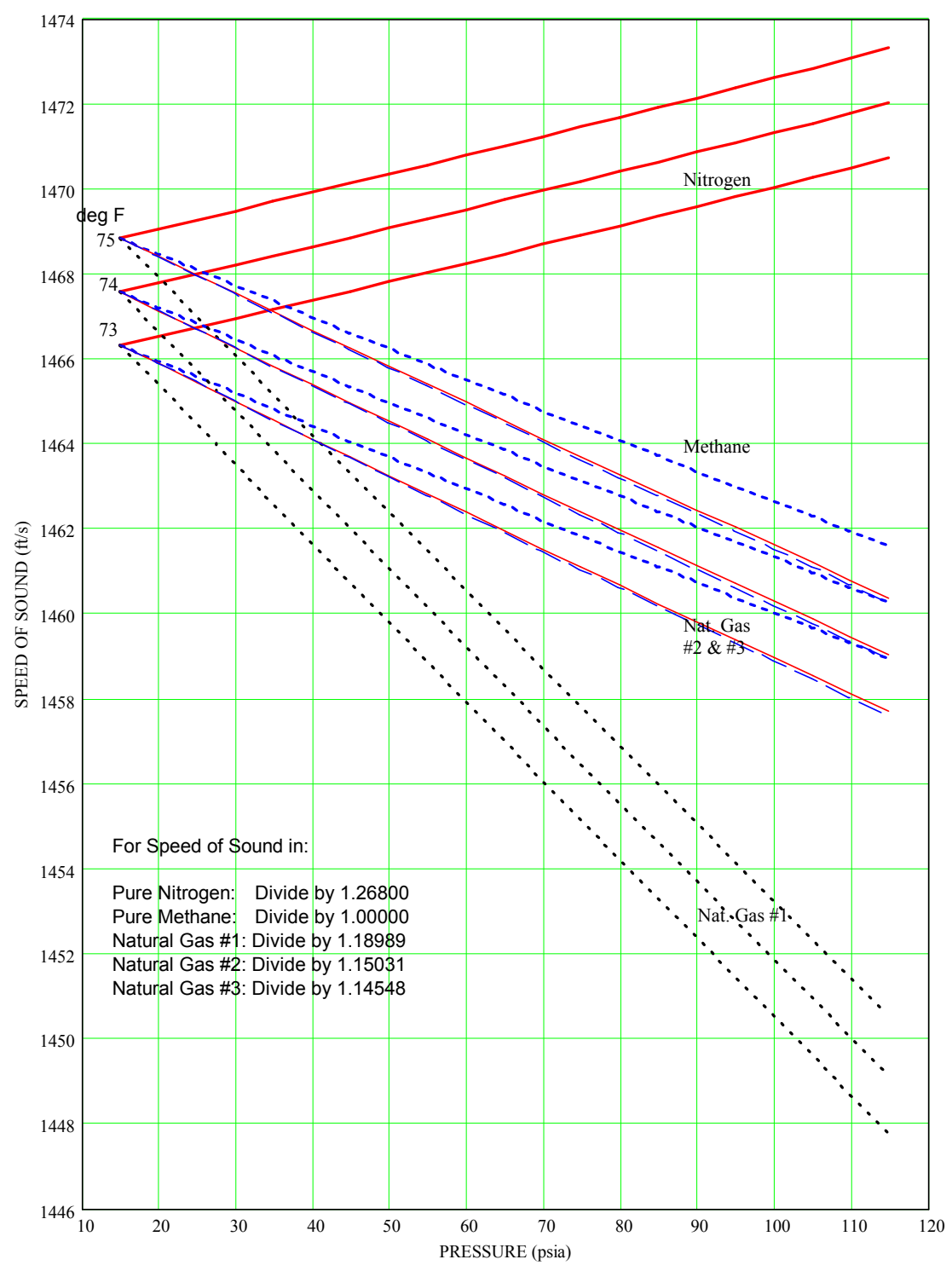

Figure A.1. Calculated speed of sound in five test gases.

[All values are scaled to the speed of sound in ultra-pure methane at 14.73 psia.] 


\section{A.5 200-kHz Transducer Tests}

Speed-of-sound measurements were performed at $200 \mathrm{kHz}$ using the Siemens DGT ultrasonic sensor installed in the test chamber with the near reflector face spaced at $2.25 \mathrm{in}$. from the transducer face. Three test gases were used: (1) Pure Nitrogen, (2) Ultra-Pure Methane, and (3) Natural Gas \#1. Table A.2 lists the pressure and temperature test conditions and the differential time of flight derived from the $200-\mathrm{kHz}$ ultrasonic signal correlation analysis for reflection faces separated at $0.9978 \mathrm{in}$. apart along the axis of the test chamber. Note: Two test runs were conducted using the nitrogen gas to demonstrate the repeatability of the experimental measurements and data analysis.

Table A.2. Test data at $200 \mathrm{kHz}$ using Siemens DGT transducer.

\begin{tabular}{|c|c|c|c|c|c|c|c|c|c|c|c|}
\hline \multicolumn{3}{|c|}{ Pure Nitrogen (Test 1) } & \multicolumn{3}{|c|}{ Pure Nitrogen (Test 2) } & \multicolumn{3}{|c|}{ Ultra-Pure Methane } & \multicolumn{3}{|c|}{ Natural Gas \#1 } \\
\hline $\begin{array}{l}\text { Press } \\
\text { (psia) }\end{array}$ & $\begin{array}{c}\text { Temp } \\
\left({ }^{\circ} \mathrm{F}\right)\end{array}$ & $\begin{array}{c}\Delta t \\
(\mu \mathrm{s})\end{array}$ & $\begin{array}{l}\text { Press } \\
\text { (psia) }\end{array}$ & $\begin{array}{c}\text { Temp } \\
\left({ }^{\circ} \mathrm{F}\right)\end{array}$ & $\begin{array}{c}\Delta t \\
(\mu s)\end{array}$ & $\begin{array}{l}\text { Press } \\
\text { (psia) }\end{array}$ & $\begin{array}{c}\text { Temp } \\
\left({ }^{\circ} \mathrm{F}\right)\end{array}$ & $\begin{array}{c}\Delta t \\
(\mu \mathrm{t})\end{array}$ & $\begin{array}{l}\text { Press } \\
\text { (psia) }\end{array}$ & $\begin{array}{c}\text { Temp } \\
\left({ }^{\circ} \mathrm{F}\right)\end{array}$ & $\begin{array}{c}\Delta t \\
(\mu \mathrm{s})\end{array}$ \\
\hline 14.266 & 10.11 & 144.000 & $1+.201$ & 10.10 & 144.040 & 14.101 & 1 1 & 117.588 & 10.705 & 10.01 & 129.547 \\
\hline 22.567 & 75.20 & 144.386 & 21.205 & 15.63 & 144.306 & 22.402 & 75.63 & 117.948 & 23.366 & 13.01 & 129.507 \\
\hline 30.453 & 75.18 & 144.306 & 29.641 & 75.70 & 144.266 & 30.169 & 74.53 & 118.028 & 30.866 & 73.29 & 129.587 \\
\hline 40.766 & 75.18 & 144.266 & 40.661 & 75.59 & 144.226 & 40.239 & 74.60 & 118.188 & 41.019 & 73.16 & 129.707 \\
\hline 49.975 & 75.16 & 144.226 & 49.886 & 75.61 & 144.186 & 50.441 & 74.44 & 118.228 & 51.464 & 73.17 & 135.027 \\
\hline 60.724 & 74.14 & 144.186 & 60.166 & 75.41 & 144.146 & 61.125 & 74.32 & 118.308 & 60.939 & 73.04 & 135.147 \\
\hline 70.929 & 10.21 & 144.146 & 70.339 & 10.40 & 144.106 & 70.700 & 4.0 & 118.388 & 70.631 & 72.98 & 135.347 \\
\hline$T_{\text {avg }}$ & 75.177 & & $T_{\text {Tyon }}$ & 75.586 & & $\mathrm{~T}_{\mathrm{av}}$ & 74.689 & & $T_{\text {avg }}$ & 73.246 & \\
\hline
\end{tabular}

Speed of Sound in Nitrogen: Figure A.2 shows the experimentally measured speed of sound versus pressure in nitrogen for the two test runs (Pure Nitrogen Test 1 and Test 2). Also shown in this figure are calculated speed-of-sound values adjusted to correspond to the average temperature of the ultrasonic test chamber. The repeated experimental test runs yield speed-ofsound values that are within $0.32 \mathrm{ft} / \mathrm{s}$ at the 40 -psia test pressure before correcting for the difference in average chamber temperature for the two test runs. With the temperature correction applied (i.e., whereby the experimental speed of sound in Test 1 was adjusted to the temperature of the experimental speed of sound in Test 2 at the 40 psia test pressure), the two experimental values agree within $0.096 \mathrm{ft} / \mathrm{s}$. The measured speed of sound for Test 2 is lower than the calculated speed of sound for Test $2 \mathrm{by}-1.20 \mathrm{ft} / \mathrm{s}$ at the $40 \mathrm{psia}$ test pressure, corresponding to an offset of $-0.149 \%$. This offset is essentially constant over the entire pressure range of the tests. The pressure-dependent slopes of the measured speed of sound in Test 1 and Test 2 are essentially identical and are also noted to be substantially the same as the slopes of the calculated speed-of-sound curves, indicating that no anomalous pressure-dependent effects (such as velocity dispersion caused by molecular relaxation) are evident in the nitrogen test gas for ultrasonic measurements in the $200-\mathrm{kHz}$ frequency range. The offset error between the experimental and calculated speed of sound is attributed to possible combinations of ultrasonic near-field phase error, uncertainty in reflector target spacing, and waveform digital sampling resolution. 


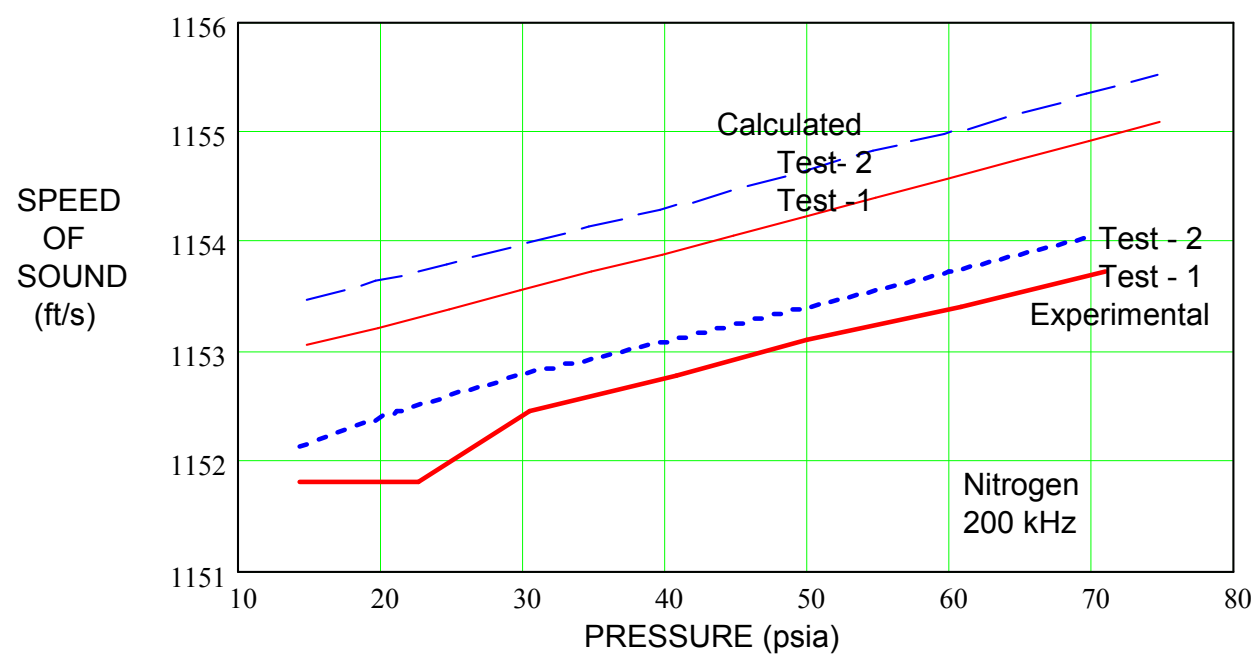

Figure A.2. Speed of sound measured at $200 \mathrm{kHz}$ in nitrogen.

Figure A.3 shows examples of the $200-\mathrm{kHz}$ ultrasonic waveforms recorded at low, medium, and high test pressures in nitrogen. Also shown is the corresponding correlation function derived from these waveforms. Not indicated to scale in these illustrations are the relative amplitudes of the waveforms that vary in approximate direct proportion with the test pressure as a result of increasing acoustic coupling with increasing gas density. The relatively long time duration of the oscillatory waveforms (10 or more periods) is indicative of the restricted transducer bandwidth (approximately $20 \mathrm{kHz}$ ) of the received ultrasonic signals. This bandwidth limitation causes the correlation functions to have a broad envelope without a welldefined maximum coherence lobe.

Speed of Sound in Methane: Figure A.4 shows the experimental speed-of-sound test results obtained at $200 \mathrm{kHz}$ in ultra-pure methane. The calculated speed of sound adjusted for the average temperature of the ultrasonic test chamber is also shown for comparison. The experimental speed of sound at the 40-psia test pressure is lower than the calculated value by $-59.5 \mathrm{ft} / \mathrm{s}$, corresponding to an offset of $-4.1 \%$. The time-of-flight difference between the measured and calculated values is $4.79 \mu \mathrm{s}$, which closely corresponds with one period of the $200-\mathrm{kHz}$ ultrasonic wave $(5.0 \mu \mathrm{s})$. Time displacement errors of one period can occur when the peak values of two adjacent lobes of the cross-correlation function are not uniquely distinguishable in the numerical data analysis. The dashed line in Figure A.4 shows the experimental speed-of-sound curve adjusted for a 5- $\mu$ s offset in measured time of flight. The adjusted experimental value at the 40-psia test pressure is $5.1 \mathrm{ft} / \mathrm{s}$ greater than the calculated value, providing a reasonable explanation for the relatively large $(-59.5 \mathrm{ft} / \mathrm{s})$ offset error. The pressure-dependent slope of the measured speed-of-sound curve is essentially identical to that of the calculated curve at pressures above about 40 psia. The measured speed of sound at pressures below 40 psia increases faster with decreasing pressure than the calculated value, indicating either that there may be a supplemental pressure dependence in methane other than that accounted for in the speed-of-sound calculations (i.e., possible molecular relaxation effects) or that the accuracy of the time-of-flight analysis may be affected by the decreasing signal-to-noise ratio of the ultrasonic pulse signal at lower pressures. 

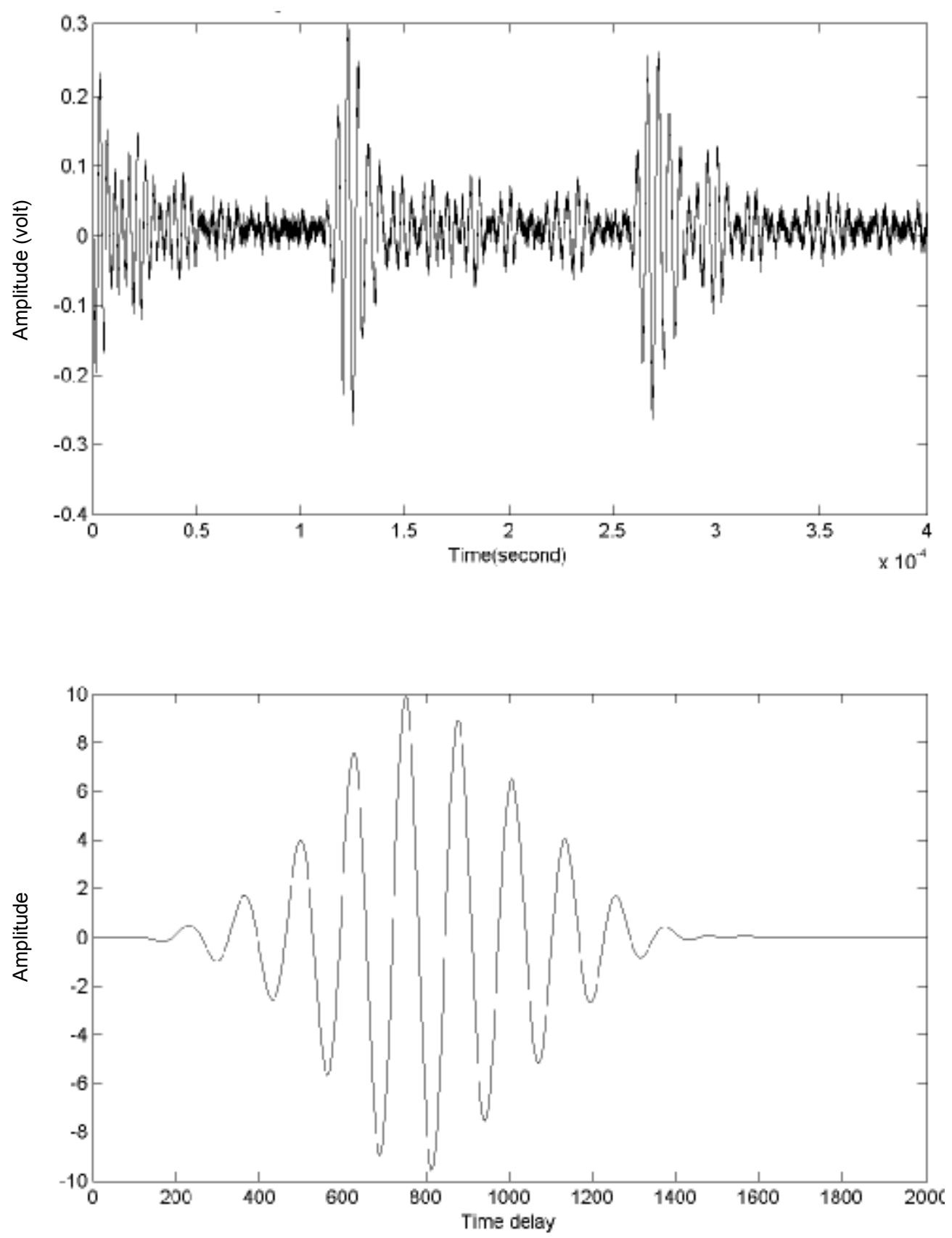

(a) Pressure: 14.27 psia

Figure A.3. Example of Siemens DGT 200-kHz ultrasonic waveforms measured in nitrogen and associated cross-correlation function [Test 1]. 

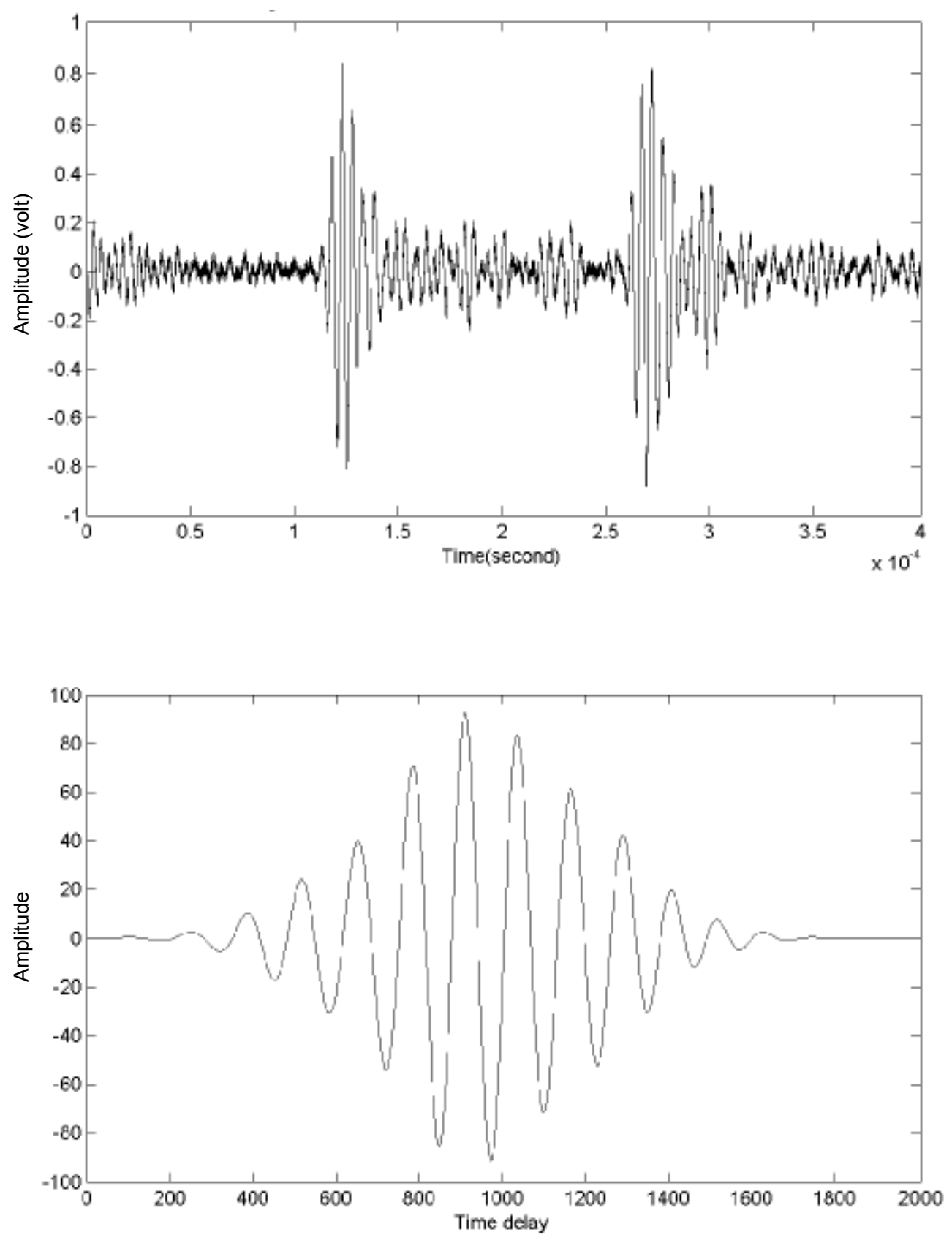

(b) Pressure: 40.77 psia

Figure A.3. Example of Siemens DGT 200-kHz ultrasonic waveforms measured in nitrogen and associated cross-correlation function [Test 1]. (continued) 

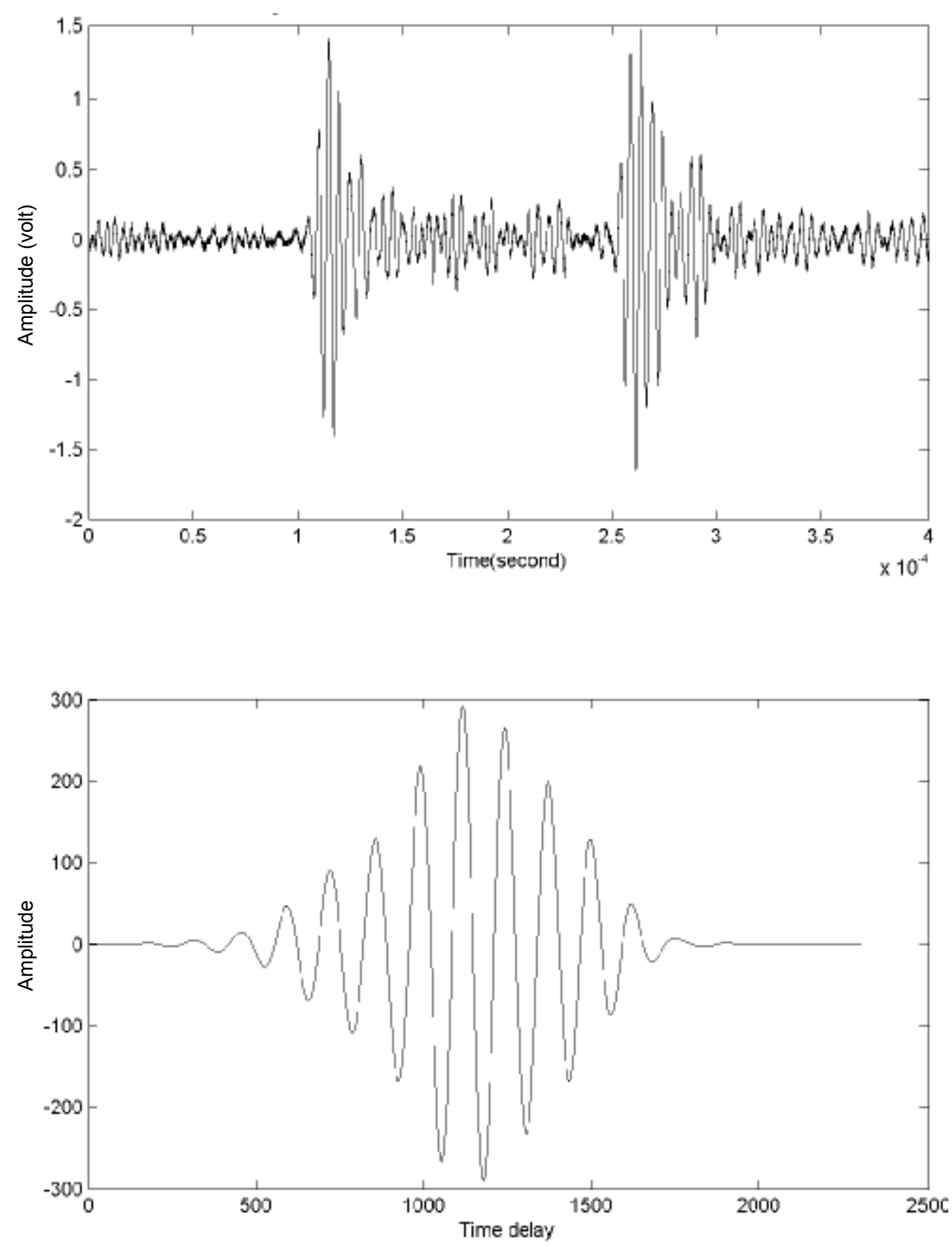

(c) Pressure: 70.93 psia

Figure A.3. Example of Siemens DGT 200-kHz ultrasonic waveforms measured in nitrogen and associated cross-correlation function [Test 1]. (continued) 


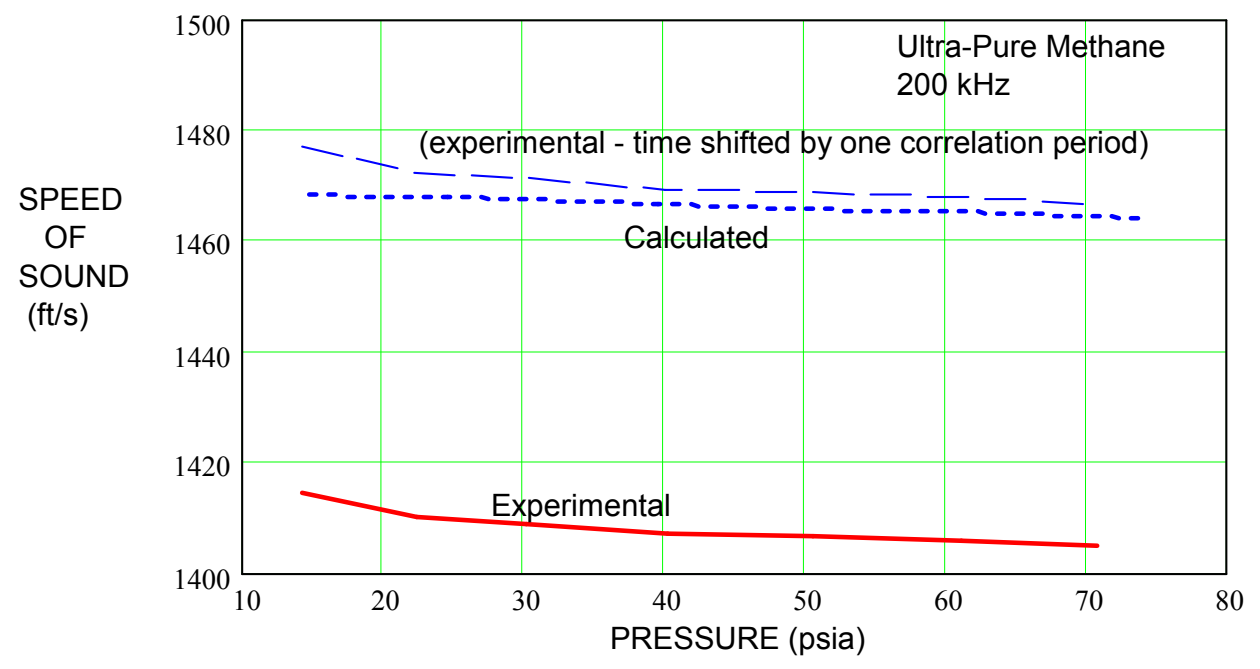

Figure A.4. Speed of sound measured at $200 \mathrm{kHz}$ in methane.

Figure A.5 shows examples of the $200-\mathrm{kHz}$ ultrasonic waveforms recorded at low, medium, and high test pressures in ultra-pure methane. Also shown are the corresponding correlation functions derived from these waveforms. Not indicated to scale are the relative amplitudes of the waveforms, which vary in approximate direct proportion with the test pressure. The relatively long time duration of the oscillatory waveforms (10 or more periods) is indicative of the restricted transducer bandwidth (approximately $20 \mathrm{kHz}$ ) of the ultrasonic signals. This bandwidth limitation causes the correlation functions to have a broad envelope without a welldefined maximum coherence lobe. The signal-to-noise ratio of these waveforms is less than that observed in nitrogen because of the reduced ultrasonic transducer coupling efficiency in methane (proportional to ratio of molecular weights of the gases).

Speed of Sound in Natural Gas \#1: Figure A.6 shows the speed of sound versus pressure measured at $200 \mathrm{kHz}$ in Natural Gas \#1. The calculated speed of sound adjusted for the average temperature of the ultrasonic test chamber is shown for comparison. The experimental values measured at 50 psia and higher compare very closely with the calculated values, whereas at pressures below 50 psia the measured values are significantly greater than the calculated value. At 50 psia (and higher), the measured value is $-1.06 \mathrm{ft} / \mathrm{s}$ lower than the calculated value, corresponding to an offset of $-0.104 \%$. At 40 psia (and lower), the measured speed of sound is higher than the calculated value by $47.9 \mathrm{ft} / \mathrm{s}$, corresponding to an offset of $3.9 \%$. The time-offlight difference between the measured and calculated values at the 40-psia test pressure is 5.05 $\mu \mathrm{s}$, which corresponds closely with one period of the $200-\mathrm{kHz}$ ultrasonic wave $(5.0 \mu \mathrm{s})$. The light dashed line in Figure A.6 shows the experimental speed-of-sound curve adjusted for a 5- $\mu$ s offset in measured time of flight. This adjusted experimental value at the 40-psia test pressure (and lower) is found to be $+0.30 \mathrm{ft} / \mathrm{s}$ greater than the calculated value. The pressure-dependent slope of the measured speed-of-sound curve is essentially identical to that of the calculated curve at pressures of 50 psia and higher. The measured speed of sound at pressures of 40 psia and lower increases more slowly with decreasing pressure than the calculated value, indicating either that there may be a supplemental pressure dependence in Natural Gas \#1 other than that accounted for in the speed-of-sound calculations (i.e., possible molecular relaxation effects) or that the 
accuracy of the time-of-flight analysis is affected by the decreasing signal-to-noise ratio of the ultrasonic pulse signals at lower pressures.
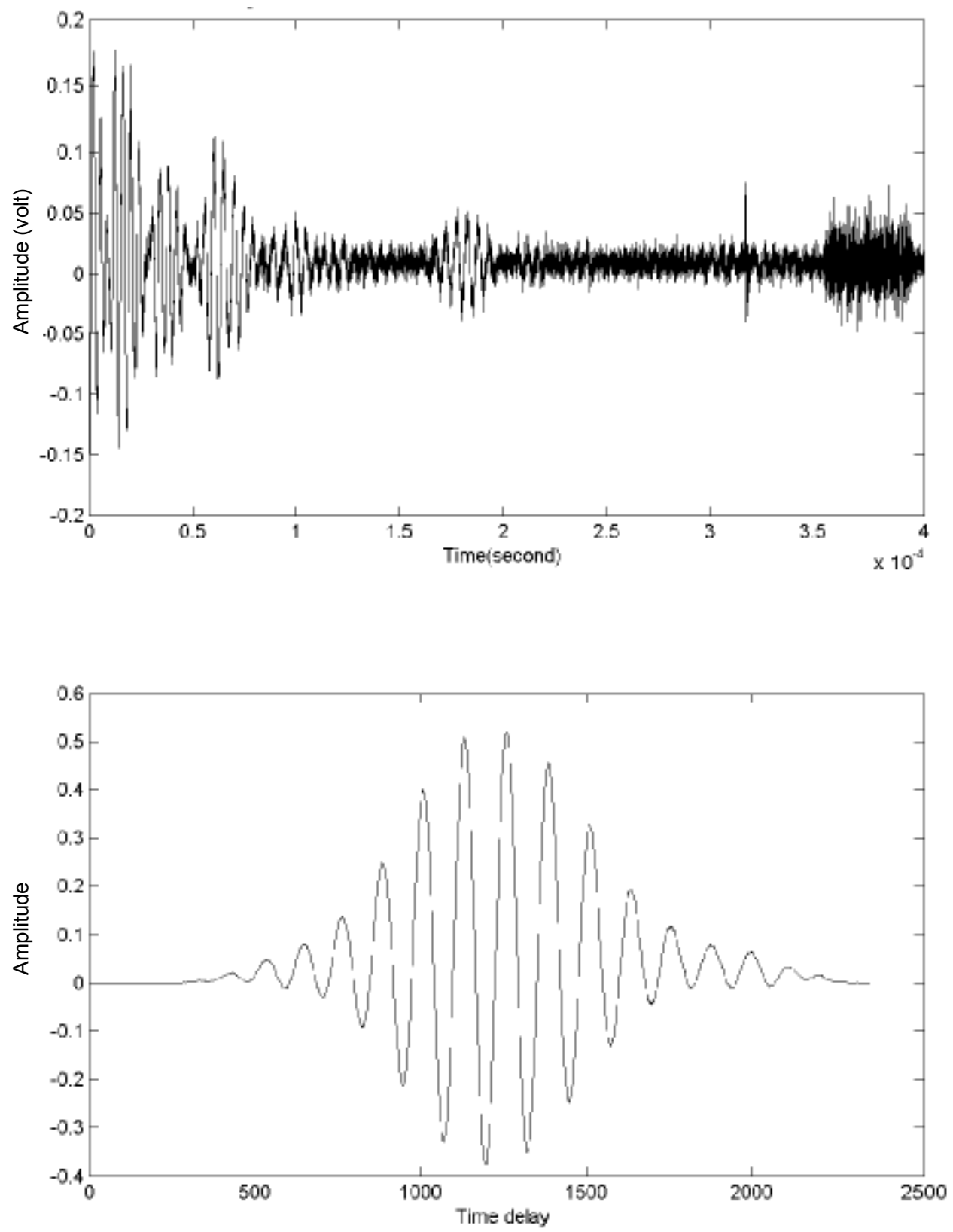

(a) Pressure : 14.19 psia

Figure A.5. Example of 200-kHz ultrasonic waveforms measured in methane and associated cross-correlation function. 

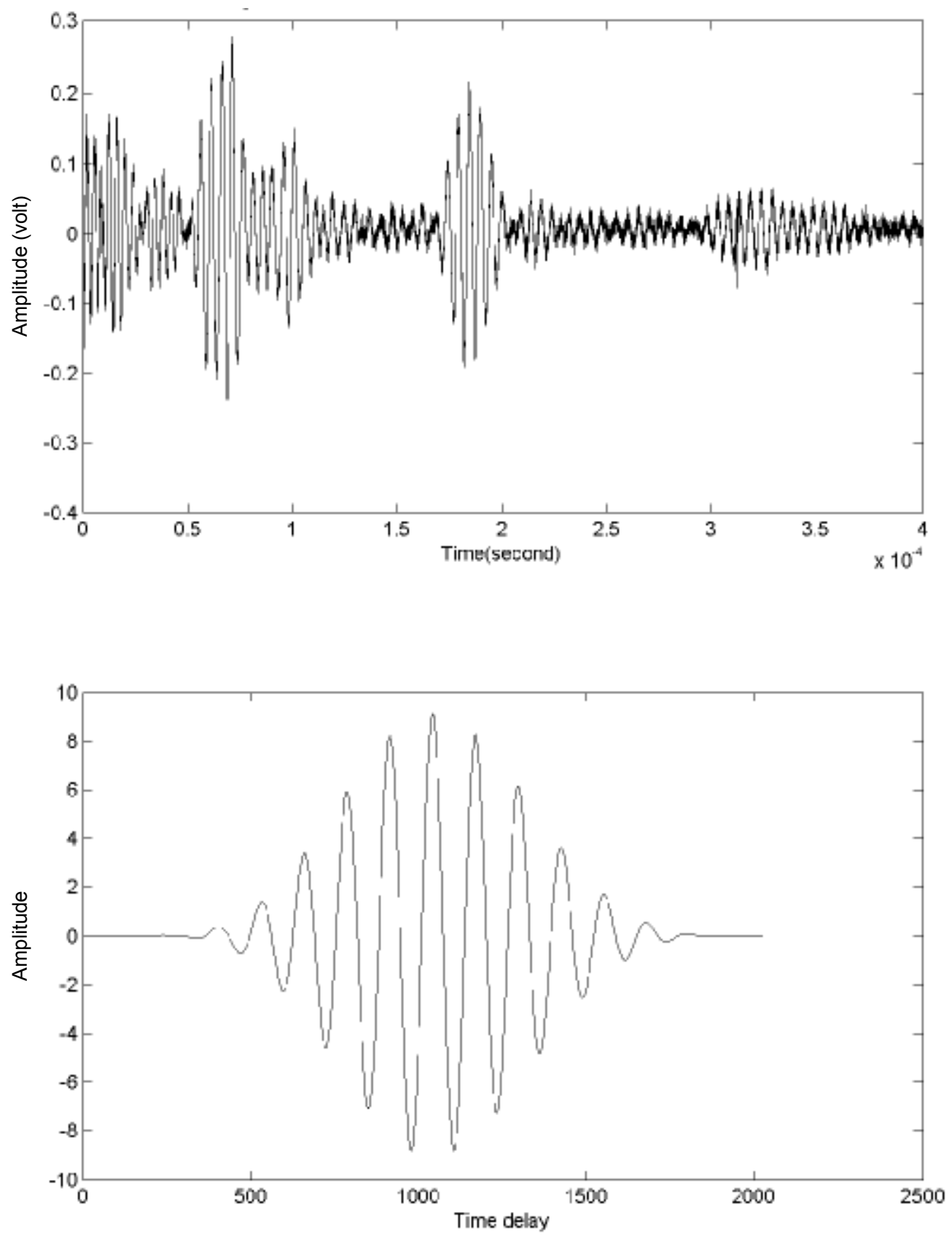

(b) Pressure : 40.24 psia

Figure A.5. Example of 200-kHz ultrasonic waveforms measured in methane and associated cross-correlation function (continued). 

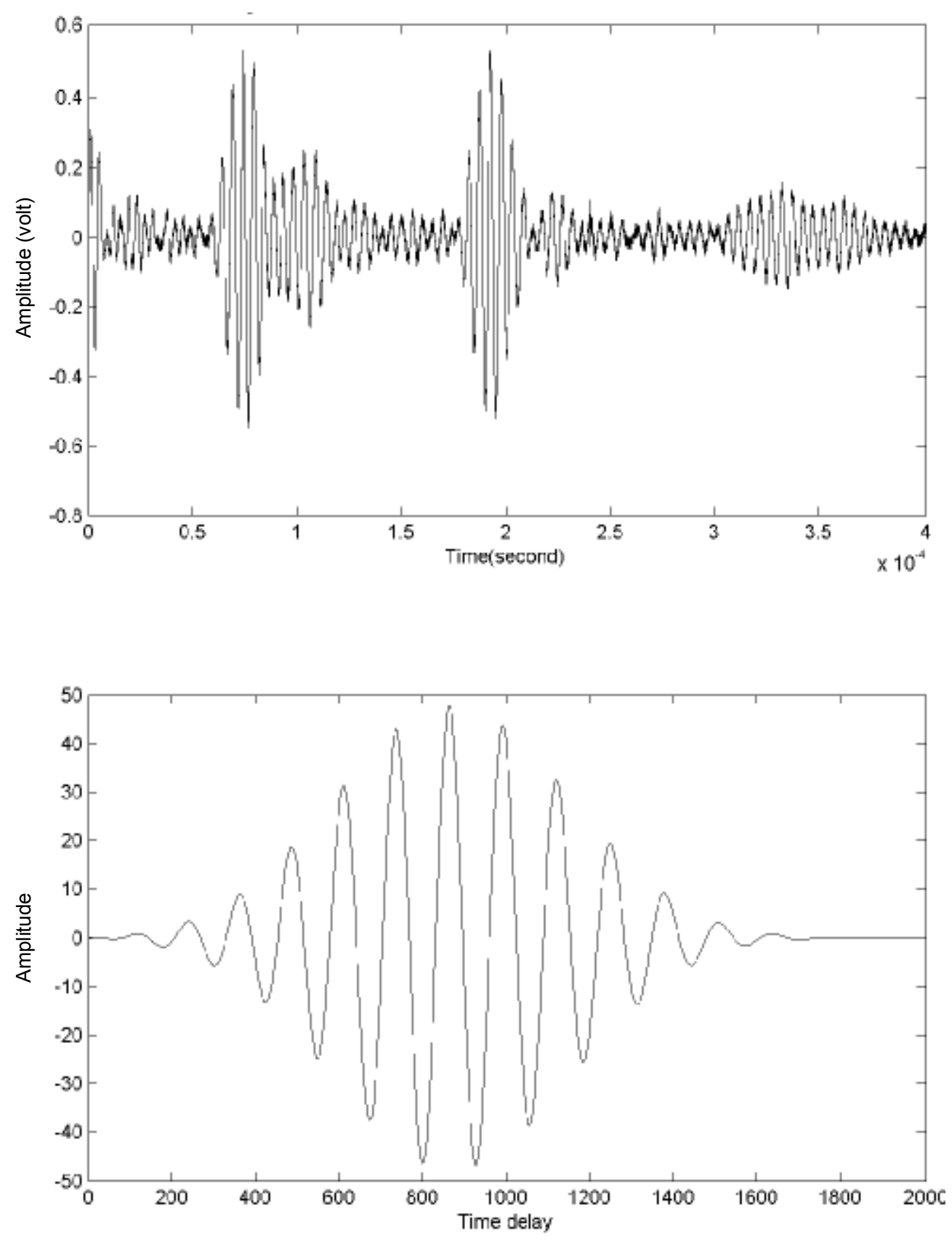

(c) Pressure: 70.70 psia

Figure A.5. Examples of $200-\mathrm{kHz}$ ultrasonic waveforms measured in methane and associated cross-correlation function (continued). 


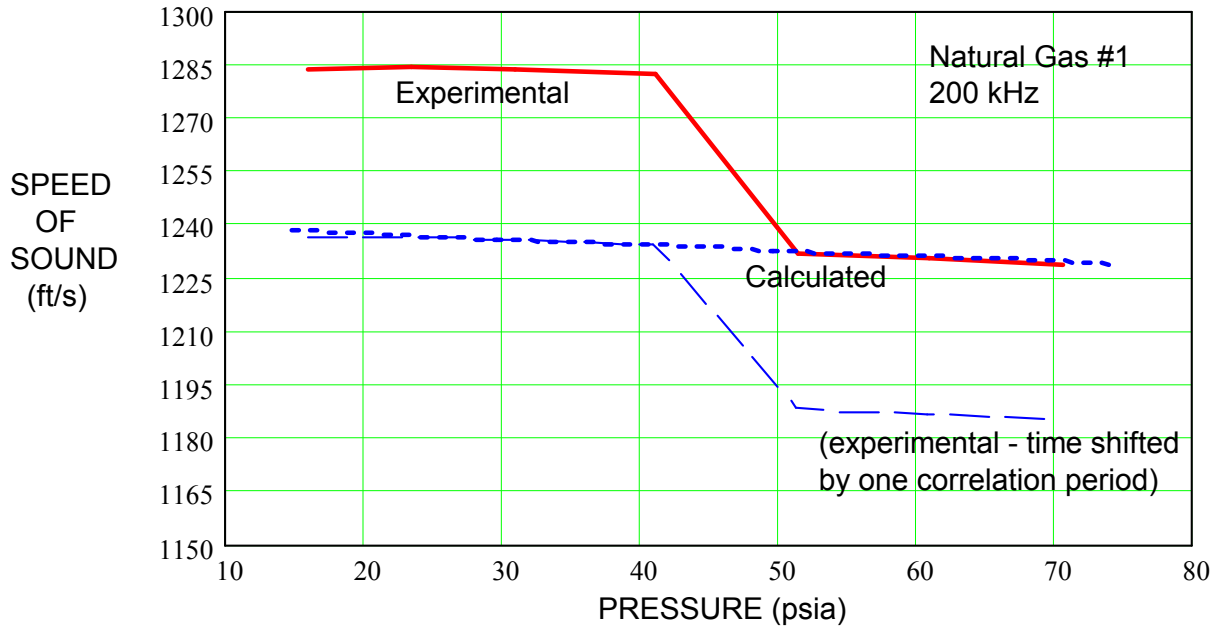

Figure A.6. Speed of sound measured at $200 \mathrm{kHz}$ in Natural Gas \#1.

Figure A.7 shows examples of the $200 \mathrm{kHz}$ ultrasonic waveforms recorded at low, medium, and high test pressures in Natural Gas \#1. Also shown are the corresponding correlation functions derived from these waveforms. Not indicated to scale in these illustrations are the relative amplitudes of the waveforms which vary in approximate direct proportion with the test pressure. The relatively long time duration of the oscillatory wave forms (10 or more periods) is indicative of the restricted transducer bandwidth (approximately $20 \mathrm{kHz}$ ) of the ultrasonic signals. This bandwidth limitation causes the correlation functions to have a broad envelope without a well-defined maximum coherence lobe. 

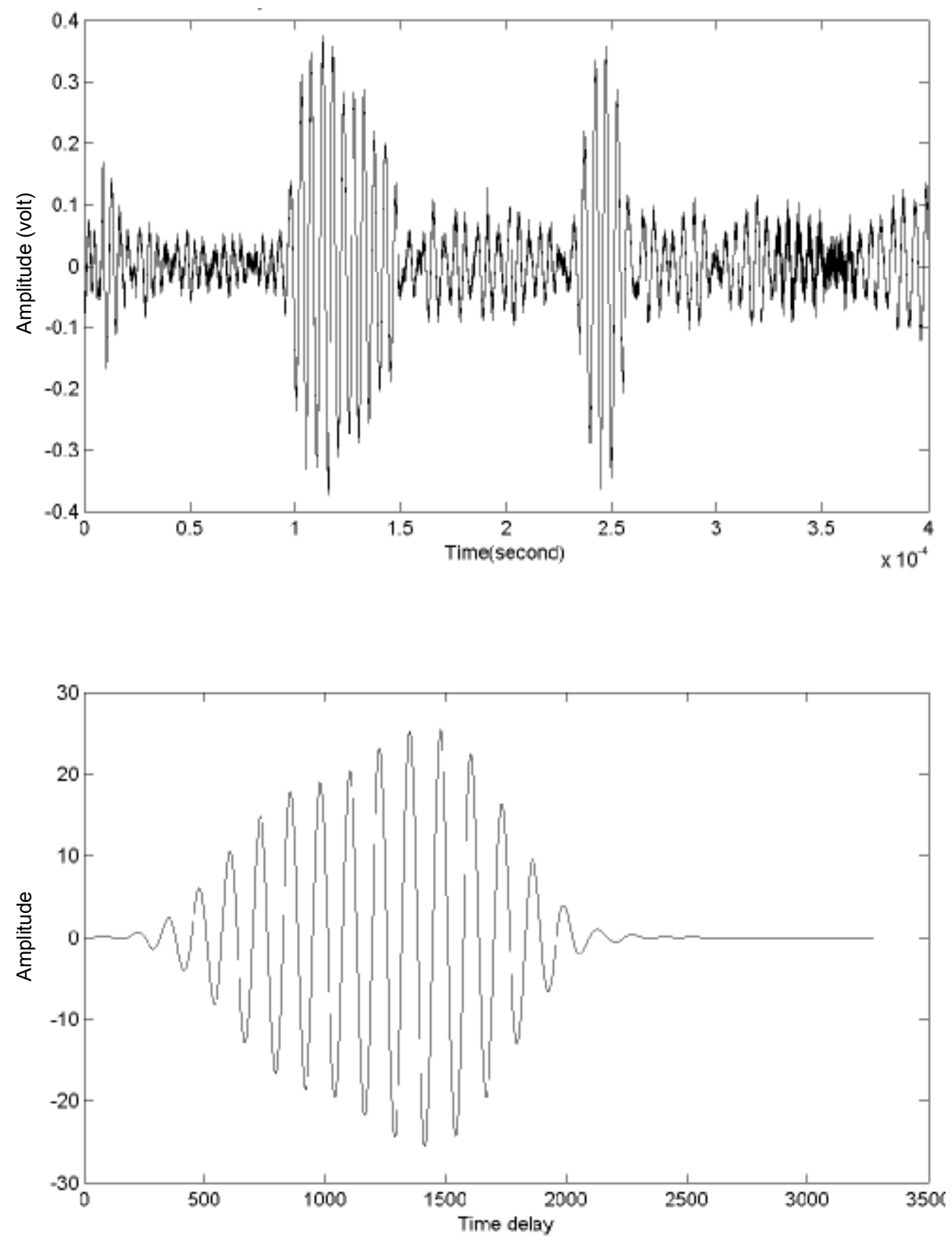

(a) Pressure: 14.44 psia

Figure A.7. Example of $200-\mathrm{kHz}$ ultrasonic waveforms measured in Natural Gas \#1 and associated cross-correlation function. 

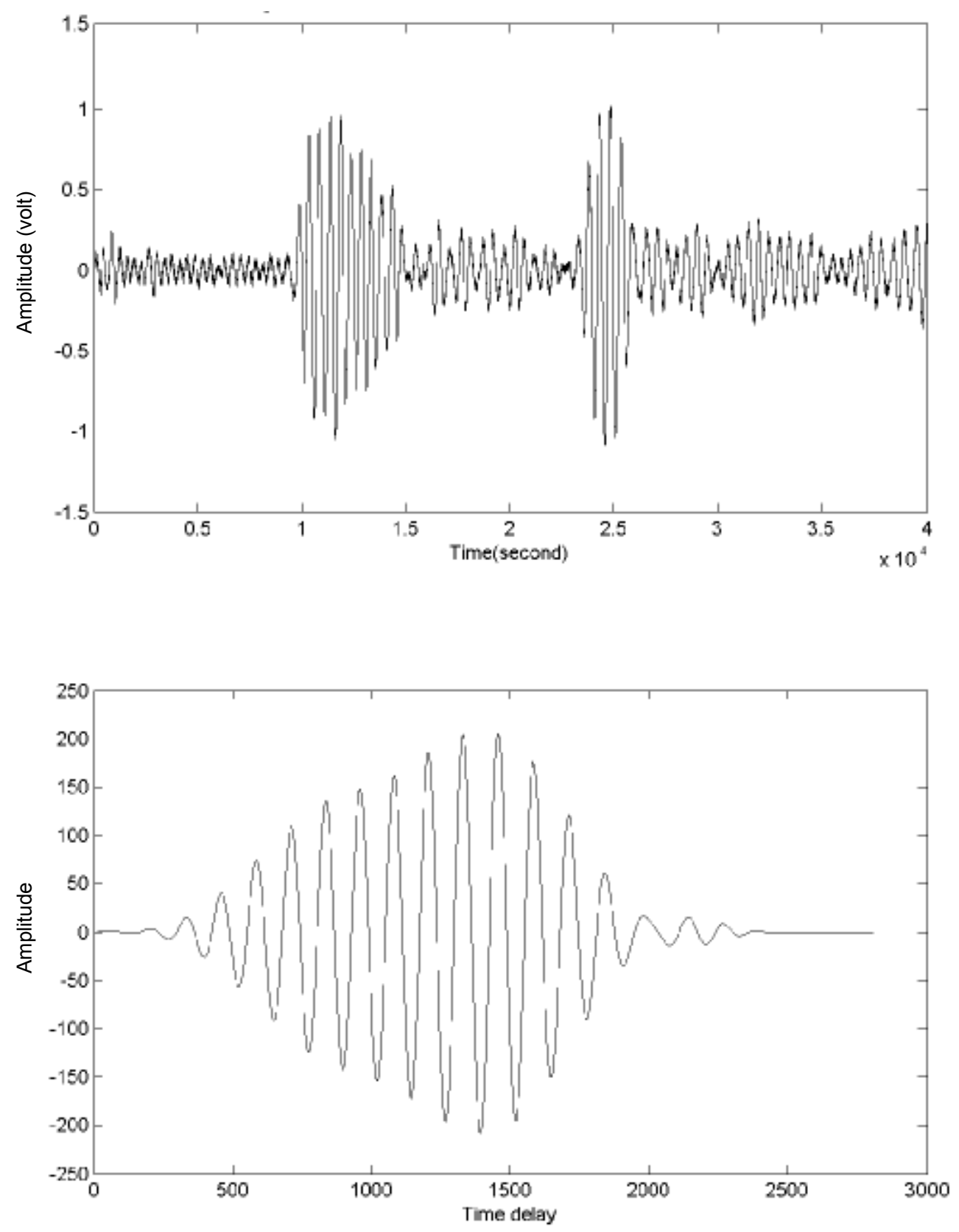

(b) Pressure: 40.28 psia

Figure A.7. Example of $200-\mathrm{kHz}$ ultrasonic waveforms measured in Natural Gas \#1 and associated cross-correlation function (continued). 

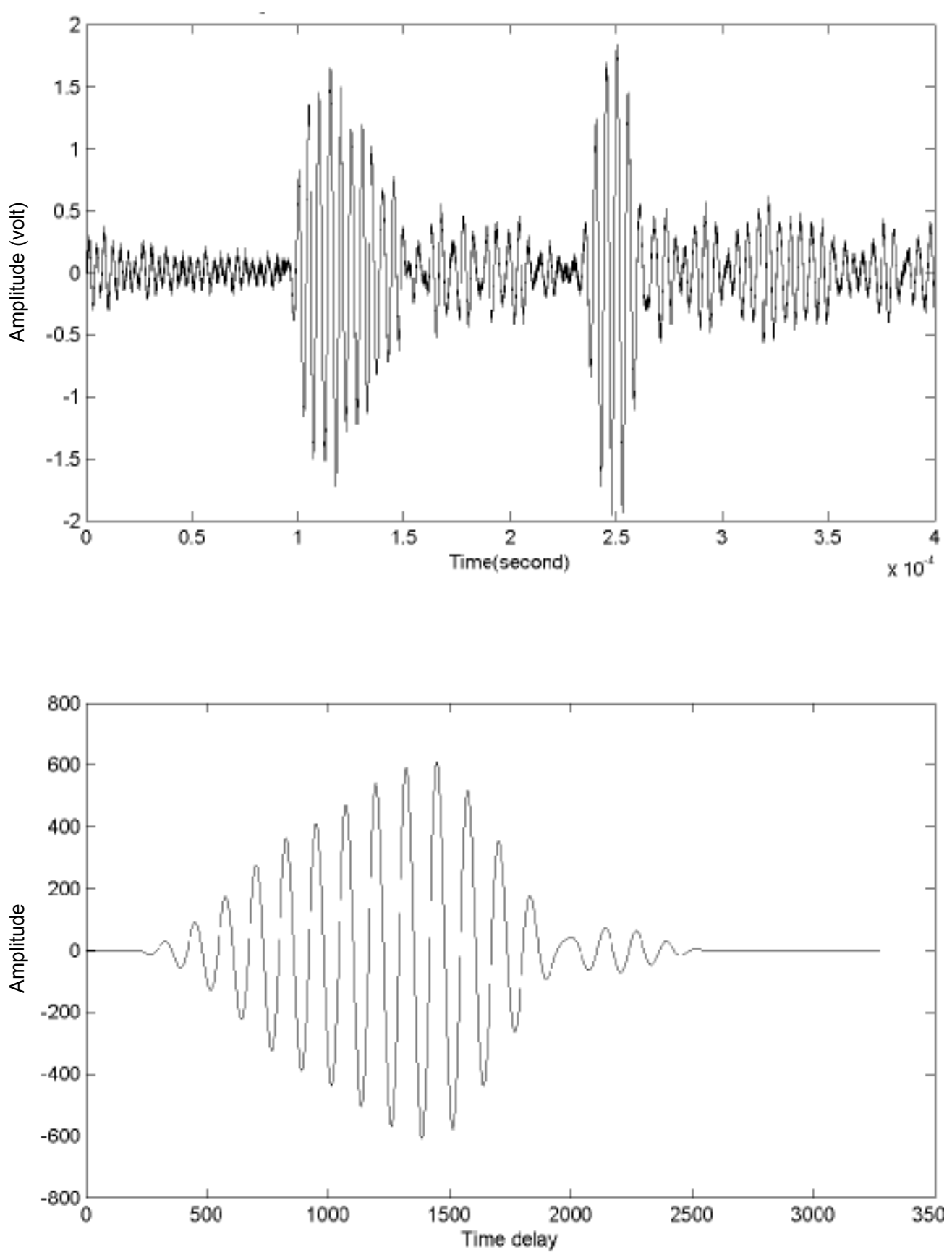

(c) Pressure: 69.35 psia

Figure A.7. Example of 200-kHz ultrasonic waveforms measured in Natural Gas \#1 and associated cross-correlation function (continued). 


\section{A.6 220-kHz Transducer Tests}

Only one speed-of-sound measurement was performed at $220 \mathrm{kHz}$ using the Massa Products Model E-188/220 ultrasonic transducer installed in the test chamber. In this test, the near reflector face was spaced a distance of 2.25 in. from the transducer face. One test gas was used: Natural Gas \#1. The Massa E-188/220 transducer was excited with a gated sine wave pulse at $200 \mathrm{kHz}$. The received pulse reflections had a very long time duration (20-30 periods) characteristic of the transducer's relatively narrow bandwidth (approximately $12 \mathrm{kHz}$ ) and a low acoustic coupling efficiency in the gas. Time-of-flight analysis of the received signals was unsuccessful in deriving a correct cross-correlation time lag for speed-of-sound measurements in Natural Gas \#1. Further evaluation tests of this transducer were discontinued.

\section{A.7 $\quad 500-k H z$ Transducer Tests}

Speed-of-sound measurements were performed at $500 \mathrm{kHz}$ using the Ultran Model NCT-105 ultrasonic transducer installed in the test chamber with the near reflector face at a distance of 3.25 in. from the transducer face. Three test gases were used: Pure Nitrogen, Natural Gas \#1, and Natural Gas \#2. Table A.3 lists the pressure and temperature test conditions and the differential time of flight derived from the $500-\mathrm{kHz}$ ultrasonic signal correlation analysis for reflection faces separated 1.0016 in. apart.

Table A.3. Test data at $500 \mathrm{kHz}$ using Ultran Model NCT-105 ultrasonic transducer.

\begin{tabular}{|c|c|c|c|c|c|c|c|c|}
\hline \multicolumn{3}{|c|}{ Pure Nitrogen } & \multicolumn{3}{|c|}{ Natural Gas \#1 } & \multicolumn{3}{|c|}{ Natural Gas \#2 } \\
\hline $\begin{array}{l}\text { Press } \\
\text { (psia) }\end{array}$ & $\begin{array}{c}\text { Temp } \\
\left({ }^{\circ} \mathrm{F}\right)\end{array}$ & $\begin{array}{c}\Delta t \\
(\mu s)\end{array}$ & $\begin{array}{l}\text { Press } \\
\text { (psia) }\end{array}$ & $\begin{array}{c}\text { Temp } \\
\left({ }^{\circ} \mathrm{F}\right)\end{array}$ & $\begin{array}{c}\Delta t \\
(\mu s)\end{array}$ & $\begin{array}{l}\text { Press } \\
\text { (psia) }\end{array}$ & $\begin{array}{c}\text { Temp } \\
\left({ }^{\circ} \mathrm{F}\right)\end{array}$ & $\begin{array}{c}\Delta t \\
(\mu \mathrm{t})\end{array}$ \\
\hline 14.266 & 75.17 & 144.386 & 14.187 & 74.71 & 117.588 & 15.909 & 73.57 & 129.547 \\
\hline 22.567 & 75.20 & 144.386 & 22.402 & 75.63 & 117.948 & 23.366 & 73.51 & 129.507 \\
\hline 30.453 & 75.18 & 144.306 & 30.169 & 74.53 & 118.028 & 30.866 & 73.29 & 129.587 \\
\hline 40.766 & 75.18 & 144.266 & 40.239 & 74.60 & 118.188 & 41.019 & 73.16 & 129.707 \\
\hline 49.975 & 75.16 & 144.226 & 50.441 & 74.44 & 118.228 & 51.464 & 73.17 & 135.027 \\
\hline 60.724 & 74.14 & 144.186 & 61.125 & 74.32 & 118.308 & 60.939 & 73.04 & 135.147 \\
\hline 70.929 & 75.21 & 144.146 & 70.700 & 74.59 & 118.388 & 70.631 & 72.98 & 135.347 \\
\hline$T_{\text {avg }}$ & 75.177 & & $T_{\text {avg }}$ & 74.689 & & $\mathrm{~T}_{\mathrm{avg}}$ & 73.246 & \\
\hline
\end{tabular}

Speed of Sound in Nitrogen: Figure A.8 shows the experimentally measured speed of sound versus pressure in nitrogen at $500 \mathrm{kHz}$. Also shown in this figure is the calculated speed of sound adjusted to correspond to the average temperature of the ultrasonic test chamber. The measured speed of sound is lower than the calculated speed of sound by $-8.49 \mathrm{ft} / \mathrm{s}$ at the 40 psia test pressure, corresponding to an offset of $-0.74 \%$. The pressure-dependent slope of the measured speed of sound differs from the slope of the calculated speed of sound in that the measured value is nearly constant over the entire range of test pressures. In particular, the 
fluctuation in the measured speed of sound in the 14-69 psia pressure range is less than $\pm 0.026 \%$, corresponding to a variation in speed of sound of only $\pm 0.30 \mathrm{ft} / \mathrm{s}$ or less. The magnitude of the offset in speed of sound $(-8.49 \mathrm{ft} / \mathrm{s})$ is only about $20 \%$ of that which would be caused by a skipped cycle in the differential time of flight derived from the correlated waveforms. A potential source of this offset error may be associated with the fact that the reflector faces are well within the near field of the $500-\mathrm{kHz}$ transducer where diffraction effects and wavefront phase differences between the two reflections could possibly introduce differential ultrasonic wave phase errors to perturb the measured speed of sound. Another possible explanation for this weak pressure dependence might be that the test chamber was not completely purged of methane gas used in the preceding test to partially nullify the decreasing speed of sound characteristic of pure nitrogen. Although Figure A.2 presented earlier shows measurements at $200 \mathrm{kHz}$, it provides a better representative determination of the measured speed of sound in nitrogen.

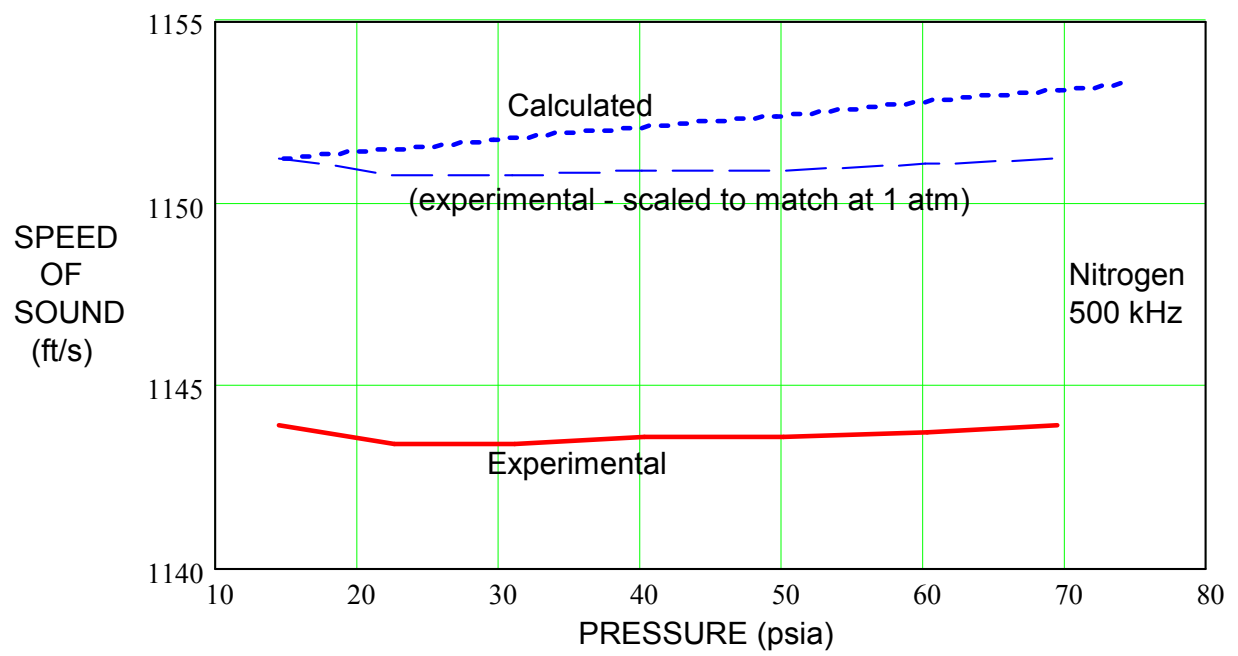

Figure A.8. Speed of sound measured at $500 \mathrm{kHz}$ in nitrogen.

Figure A.9 shows examples of the $500-\mathrm{kHz}$ ultrasonic waveforms recorded at low, medium, and high test pressures in nitrogen. Also shown are the corresponding correlation functions derived from these waveforms. Not indicated to scale in these illustrations are the relative amplitudes of the waveforms, which vary in approximate direct proportion with the test pressure. The relatively long time duration of the oscillatory waveforms (10 or more periods) is indicative of the restricted transducer bandwidth (approximately $40 \mathrm{kHz}$ ) of the ultrasonic signals. This bandwidth limitation causes the correlation functions to have a broad envelope without a well-defined maximum coherence lobe. 

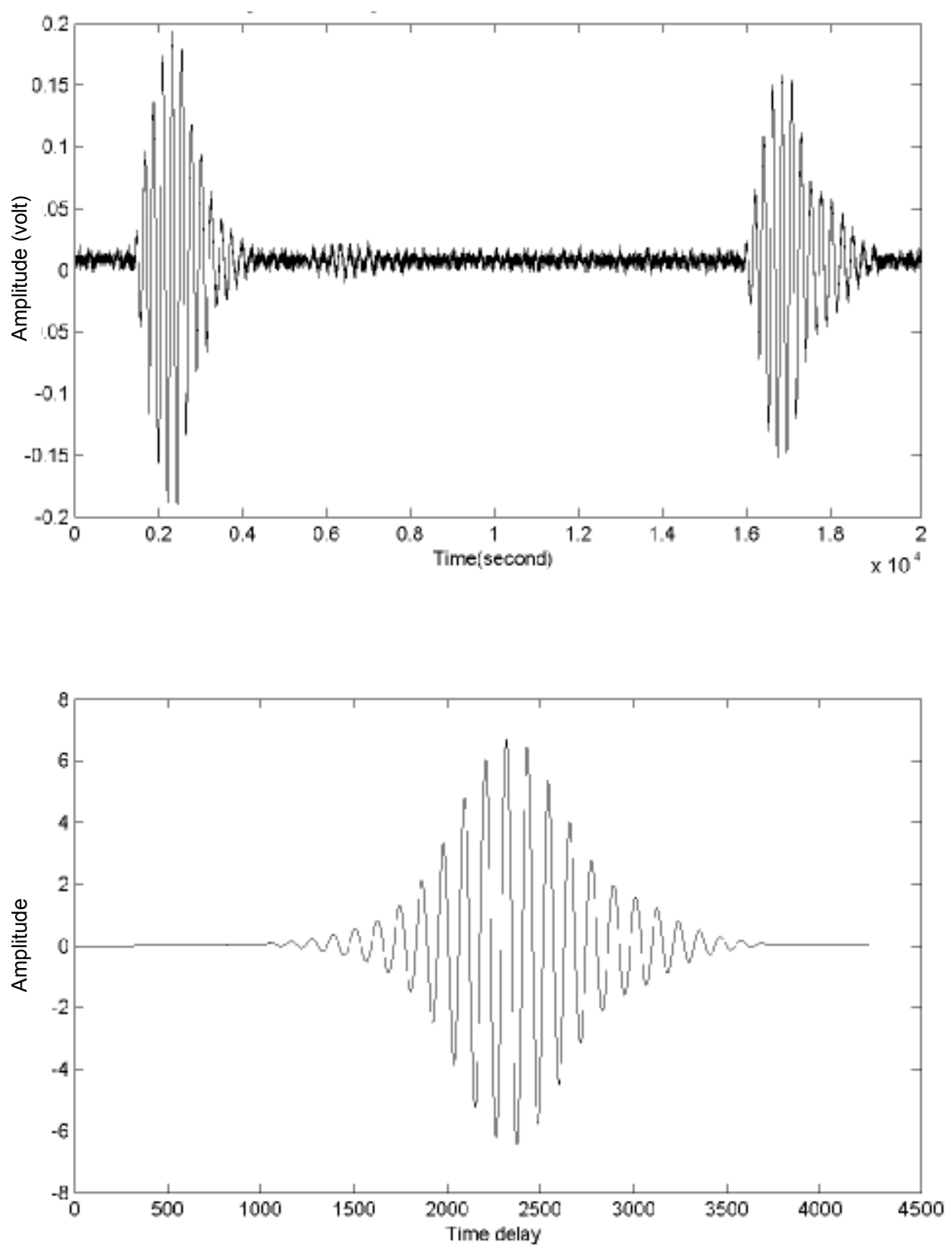

(a) Pressure: 14.44 psia

Figure A.9. Example of 500-kHz ultrasonic waveforms measured in nitrogen and associated cross-correlation function. 

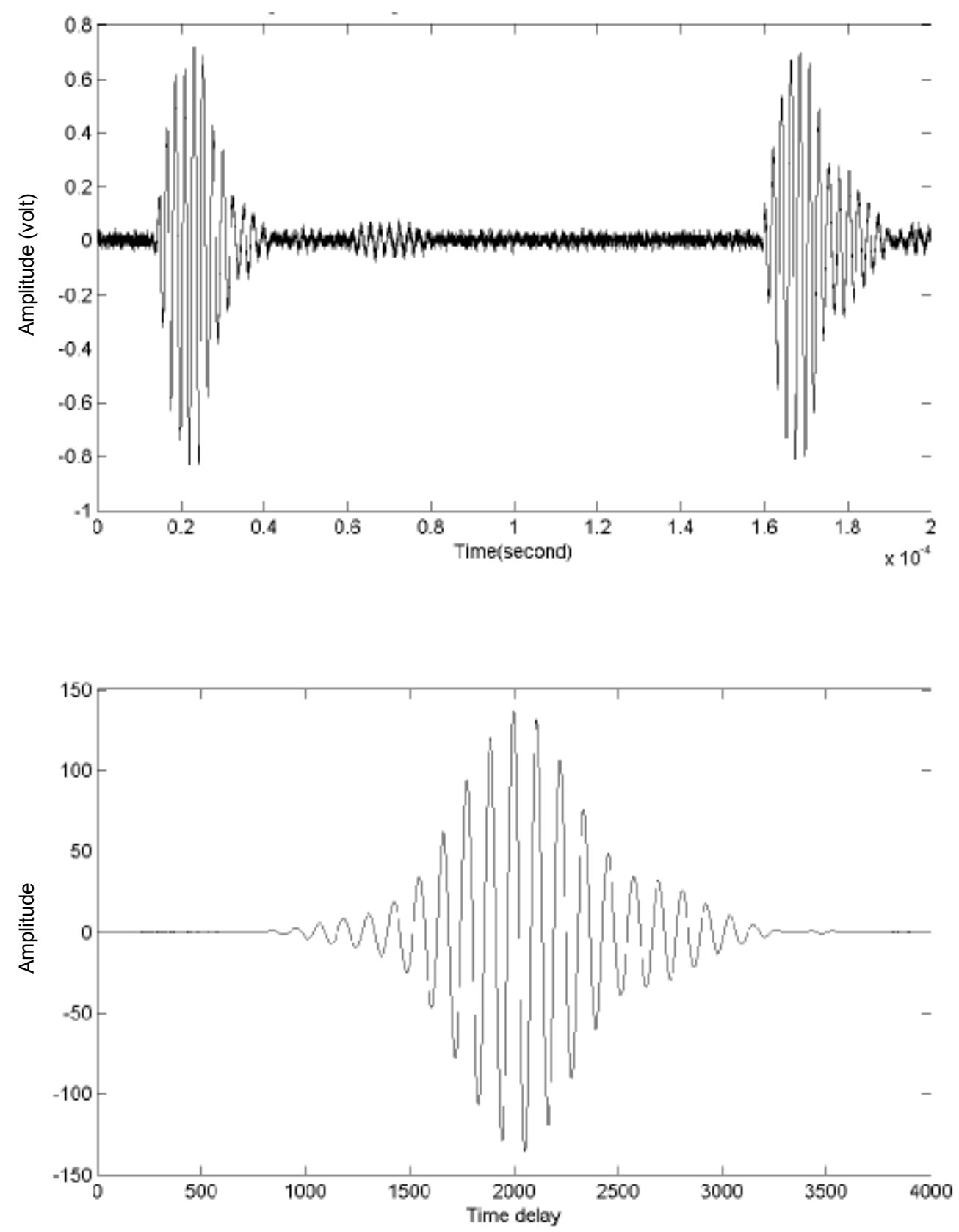

(b) Pressure: 40.24 psia

Figure A.9. Example of $500-\mathrm{kHz}$ ultrasonic waveforms measured in nitrogen and associated cross-correlation function. (continued) 

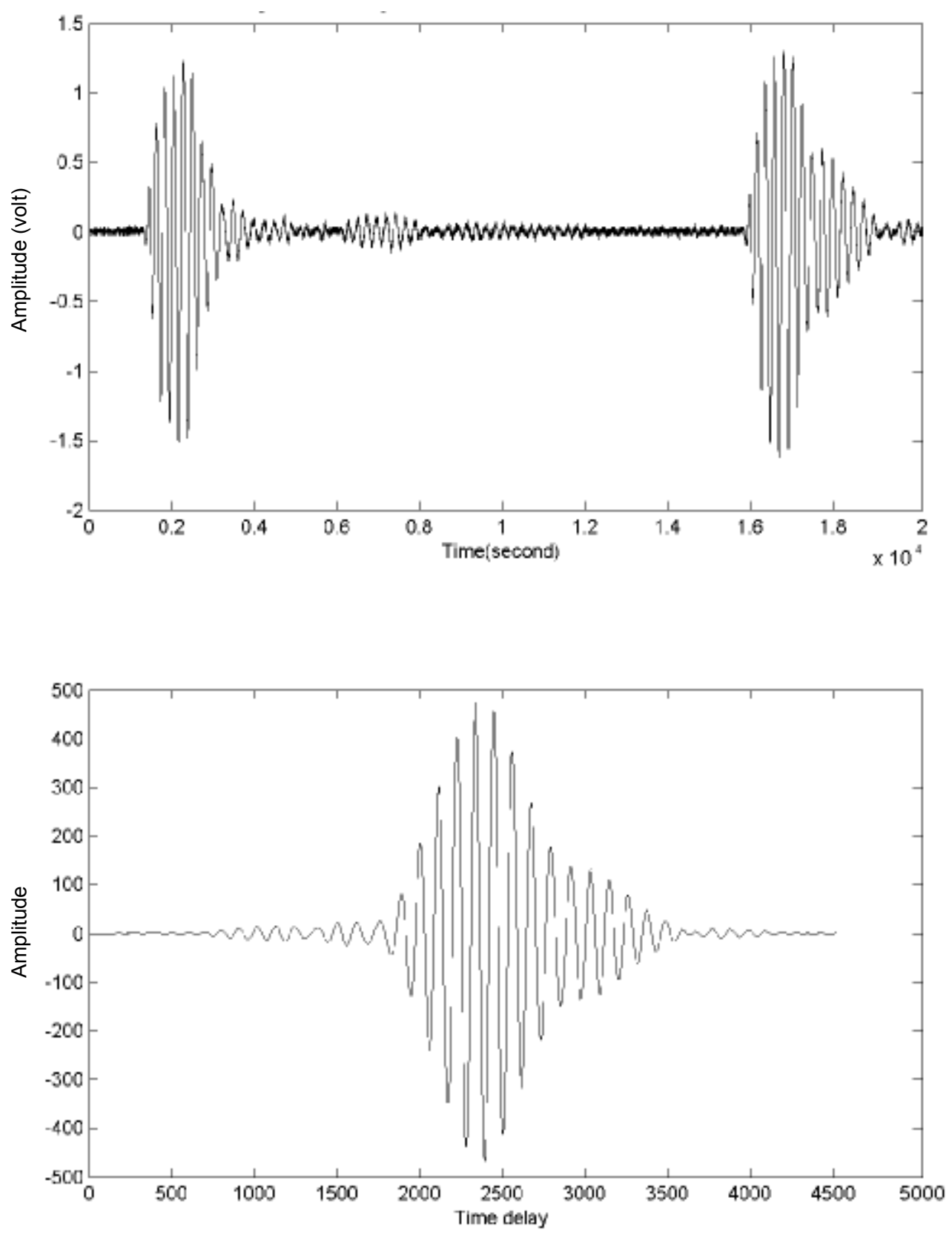

(c) Pressure: 69.35 psia

Figure A.9. Example of 500-kHz ultrasonic waveforms measured in nitrogen and associated cross-correlation function. (continued) 
Speed of Sound in Natural Gas \#1: Figure A.10 shows the experimentally measured speed of sound versus pressure in Natural Gas \#1 at $500 \mathrm{kHz}$. Also shown in this figure is the calculated speed of sound adjusted to correspond to the average temperature of the ultrasonic test chamber. The measured speed of sound is lower than the calculated speed of sound by $-4.82 \mathrm{ft} / \mathrm{s}$ at the 40 -psia test pressure, corresponding to an offset of $-0.39 \%$. The pressure-dependent slope of the measured speed of sound is essentially the same as that of the calculated curve at pressures of $50 \mathrm{psia}$ and higher. The measured speed of sound at pressures of $40 \mathrm{psia}$ and lower increases more slowly with decreasing pressure than the calculated value, corresponding to a similar effect observed at $200 \mathrm{kHz}$. The magnitude of the offset in speed of sound $(-4.82 \mathrm{ft} / \mathrm{s})$ is only about $5 \%$ of that which would be caused by a skipped cycle in the differential time-of-flight correlation function. A potential source of this offset error may be associated with the fact that the reflector faces are well inside the near field of the $500-\mathrm{kHz}$ transducer, where diffraction effects and wavefront phase differences between the two reflections could possibly introduce a differential time delay to perturb the measured speed of sound.

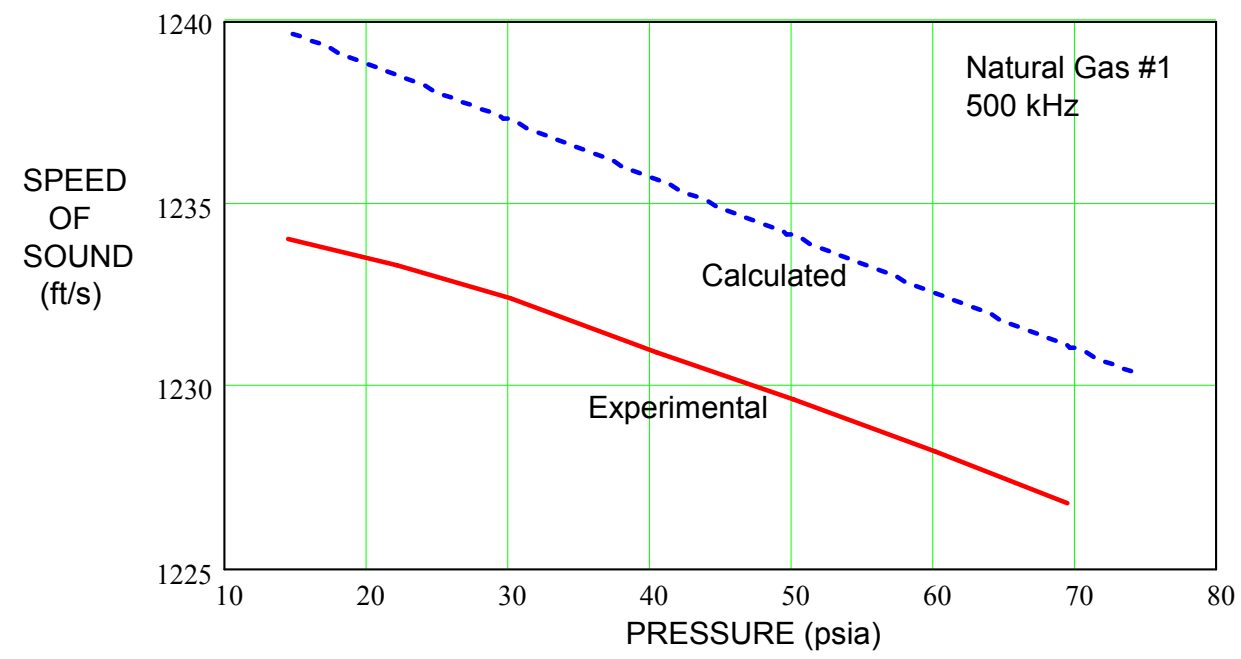

Figure A.10. Speed of sound measured at $500 \mathrm{kHz}$ in Natural Gas \#1.

Figure A.11 shows examples of the $500-\mathrm{kHz}$ ultrasonic waveforms recorded at low, medium, and high test pressures in Natural Gas \#1. Also shown are the corresponding correlation functions derived from these waveforms. Not indicated to scale in these illustrations are the relative amplitudes of the waveforms which vary in approximate direct proportion with the test pressure. The relatively long time duration of the oscillatory waveforms (10 or more periods) is indicative of the restricted transducer bandwidth (approximately $40 \mathrm{kHz}$ ) of the ultrasonic signals. This bandwidth limitation causes the correlation functions to have a broad envelope without a well-defined maximum coherence lobe. 

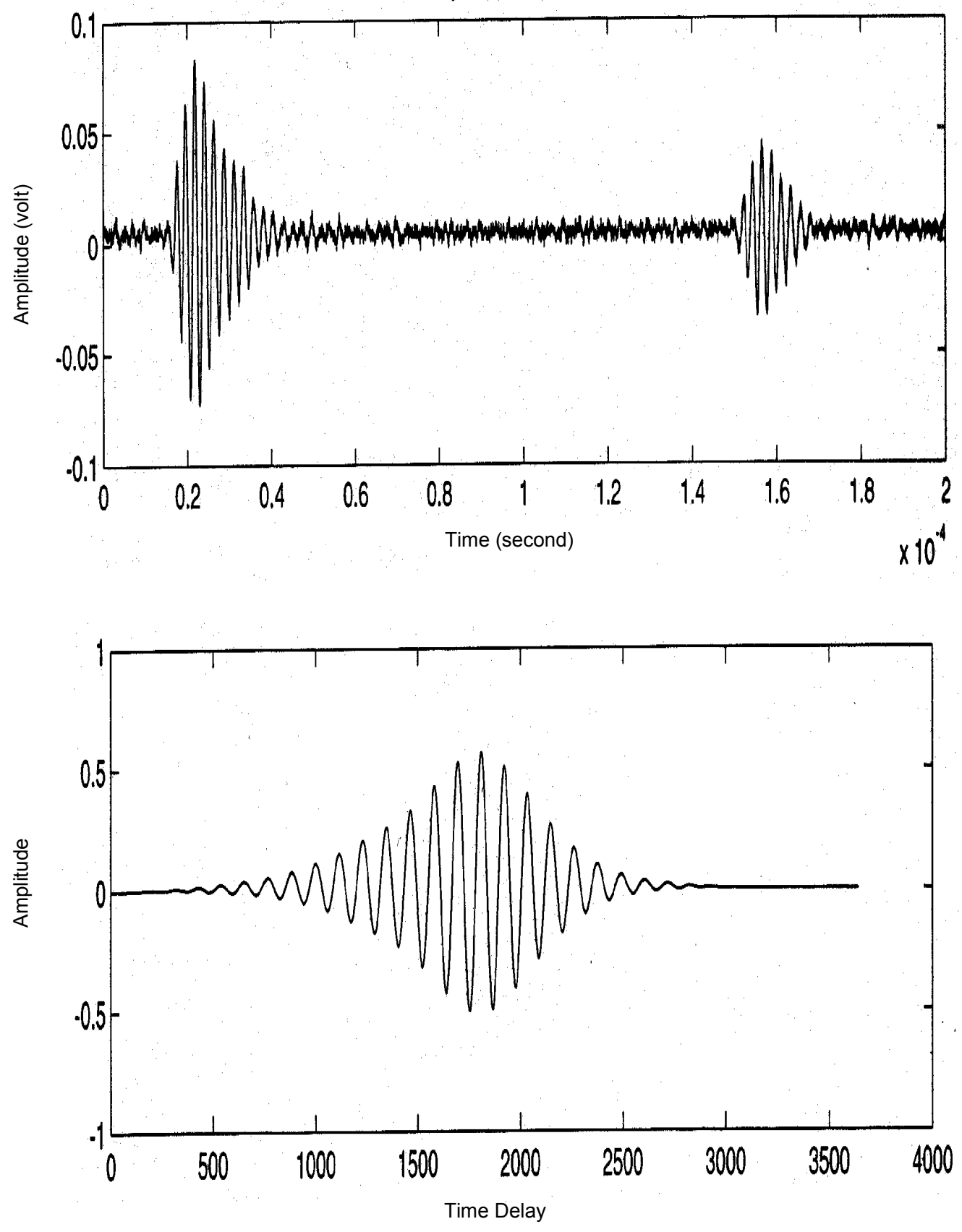

(a) Pressure: 14.38 psia

Figure A.11. Example of $500-\mathrm{kHz}$ ultrasonic waveforms measured in Natural Gas \#1 and associated cross-correlation function. 

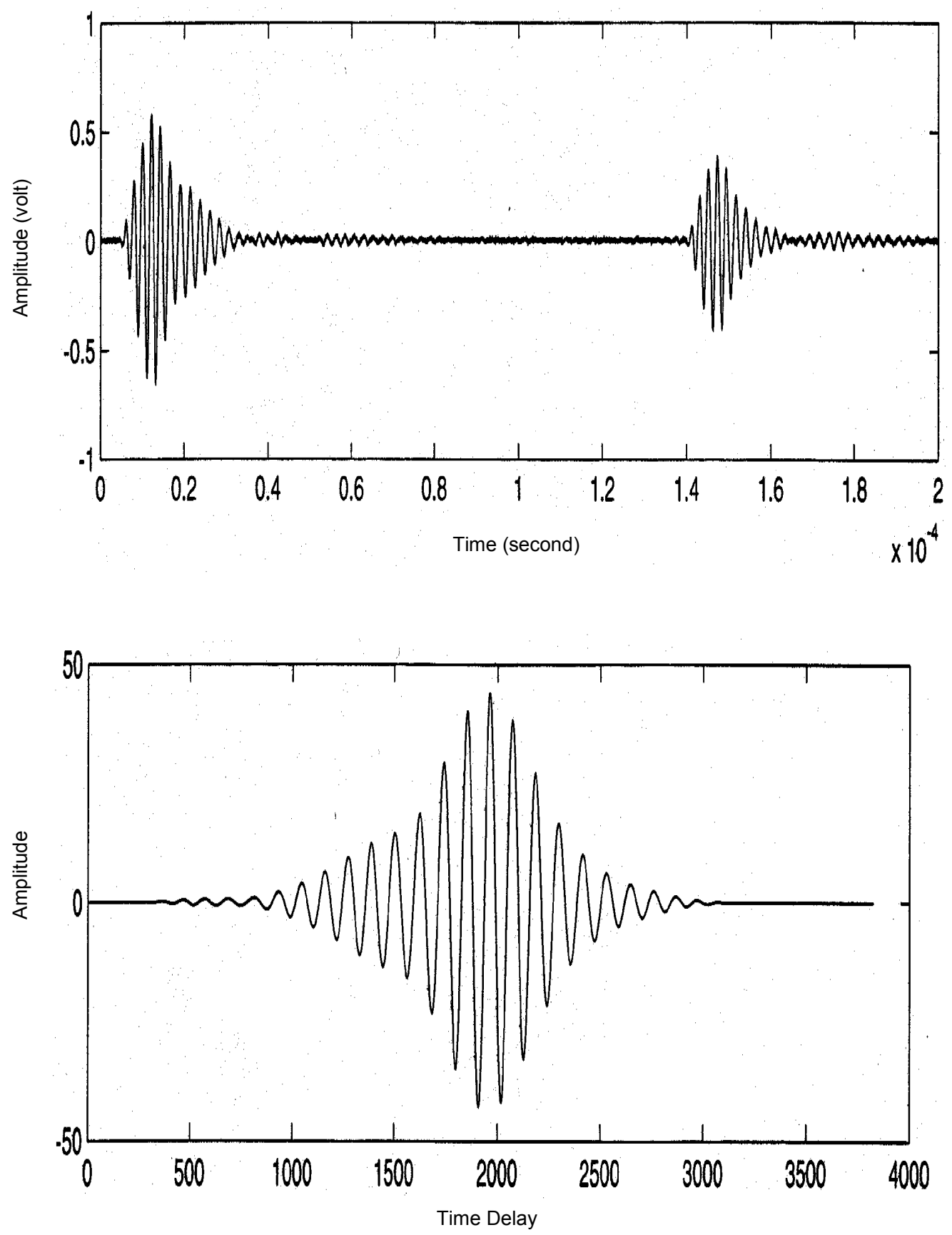

(b) Pressure: 40.39 psia

Figure A.11. Example of $500-\mathrm{kHz}$ ultrasonic waveforms measured in Natural Gas \#1 and associated cross-correlation function. (continued) 

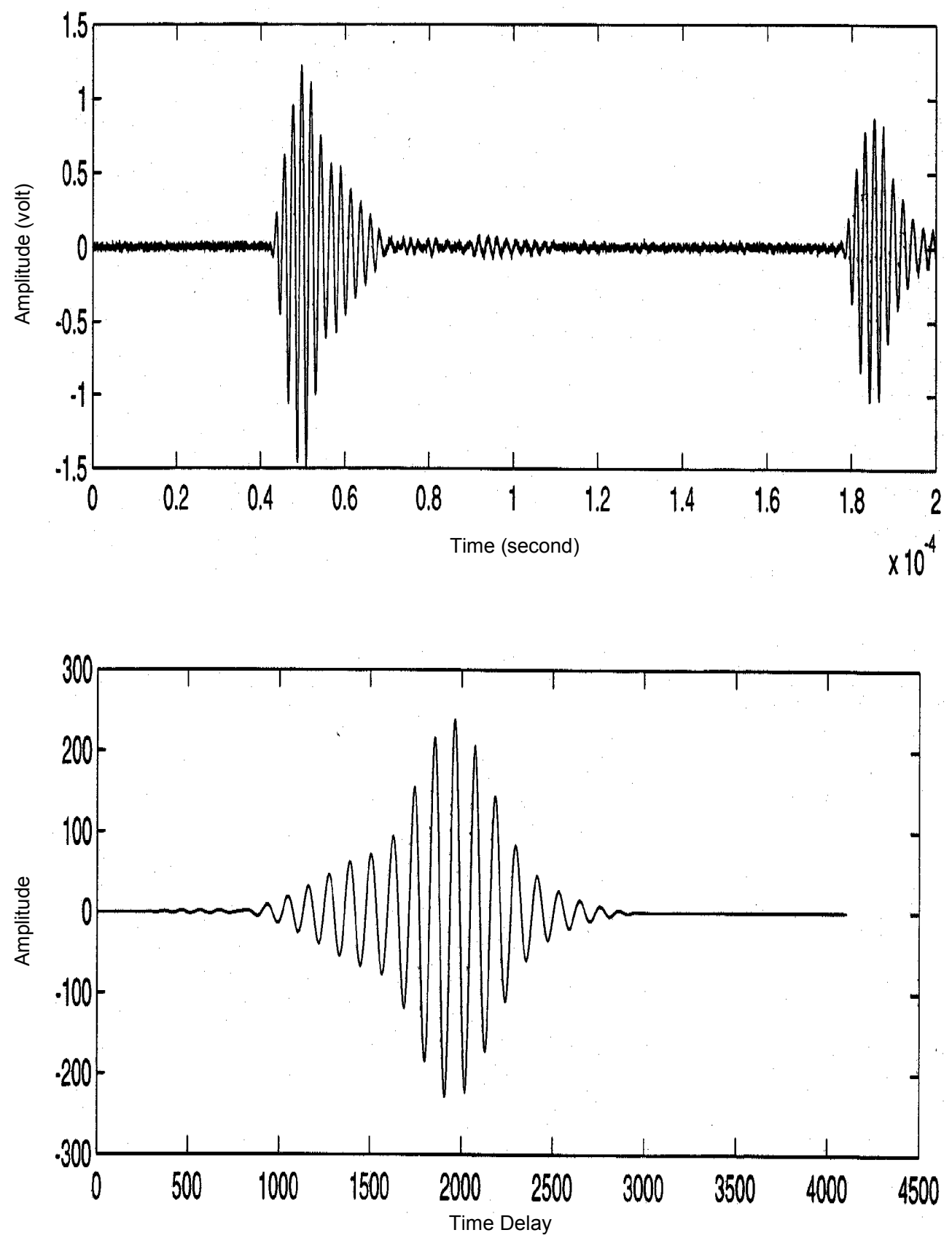

(c) Pressure: 69.44 psia

Figure A.11. Example of $500-\mathrm{kHz}$ ultrasonic waveforms measured in Natural Gas \#1 and associated cross-correlation function. (continued) 
Speed of Sound in Natural Gas \#2: Figure A.12 shows the experimentally measured speed of sound versus pressure in Natural Gas \#2 at $500 \mathrm{kHz}$. Also shown in this figure is the calculated speed of sound adjusted to correspond to the average temperature of the ultrasonic test chamber. The gas sample used in this test was contained in a 1-liter sample cylinder and, after using part of the gas to purge the test chamber, the remaining gas was insufficient to provide measurement pressures above 47.5 psia. The measured speed of sound is lower than the calculated speed of sound by $-4.01 \mathrm{ft} / \mathrm{s}$ at the 40 -psia test pressure, corresponding to an offset of $0.282 \%$. The slope of the measured speed of sound has approximately the same pressure dependence as the calculated values, but tends to decrease more slowly with increasing pressure than the slope of the calculated speed of sound. The magnitude of the offset in speed of sound $(-4.01 \mathrm{ft} / \mathrm{s})$ is only about $4.4 \%$ of that which would be caused by a skipped cycle in the differential time-of-flight correlation function. A potential source of this offset error may be associated with the fact that the reflector faces are in the near field of the $500-\mathrm{kHz}$ transducer where diffraction effects and wavefront phase differences between the two reflections could possibly introduce a differential time delay to perturb the measured speed of sound.

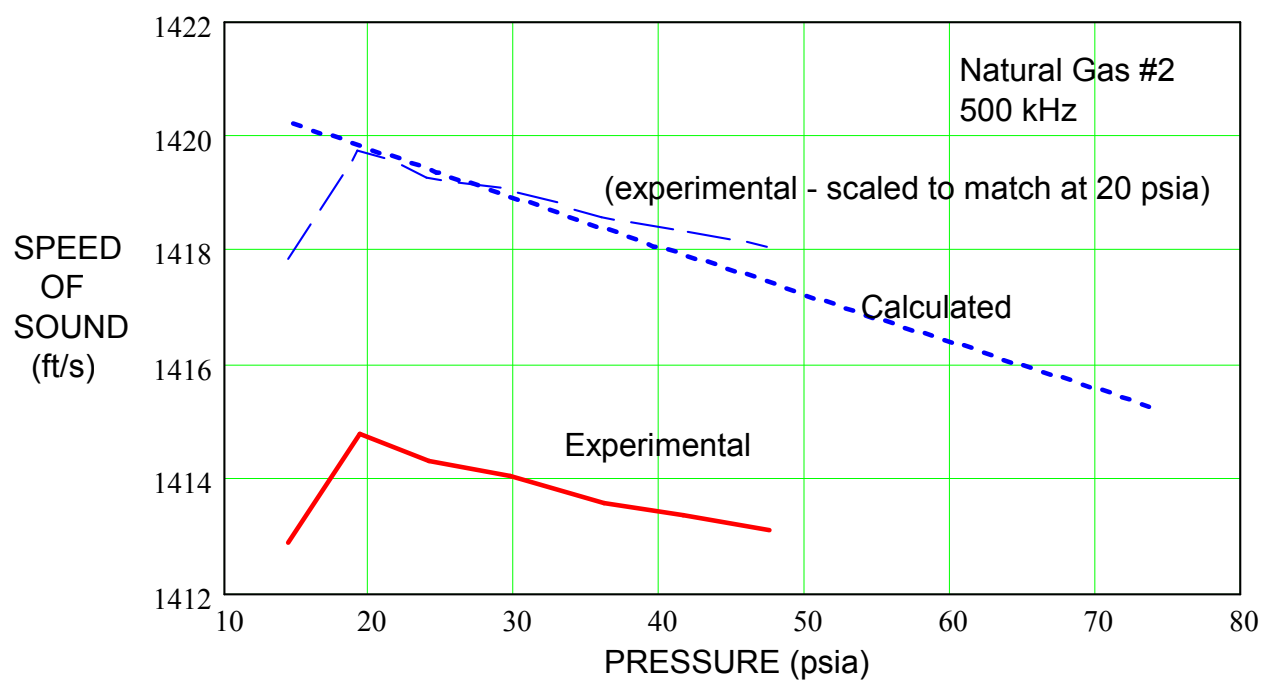

Figure A.12. Speed of sound measured at $500 \mathrm{kHz}$ in Natural Gas \#2.

Figure A.13 shows examples of the $500-\mathrm{kHz}$ ultrasonic waveforms recorded at low, medium, and high test pressures in Natural Gas \#2. Also shown are the corresponding correlation function derived from these waveforms. Not indicated to scale in these illustrations are the relative amplitudes of the waveforms, which vary in approximate direct proportion with the test pressure. The relatively long time duration of the oscillatory waveforms (10 or more periods) is indicative of the restricted transducer bandwidth (approximately $40 \mathrm{kHz}$ ) of the ultrasonic signals. This bandwidth limitation causes the correlation function to have a broad envelope without a well-defined maximum coherence lobe. 

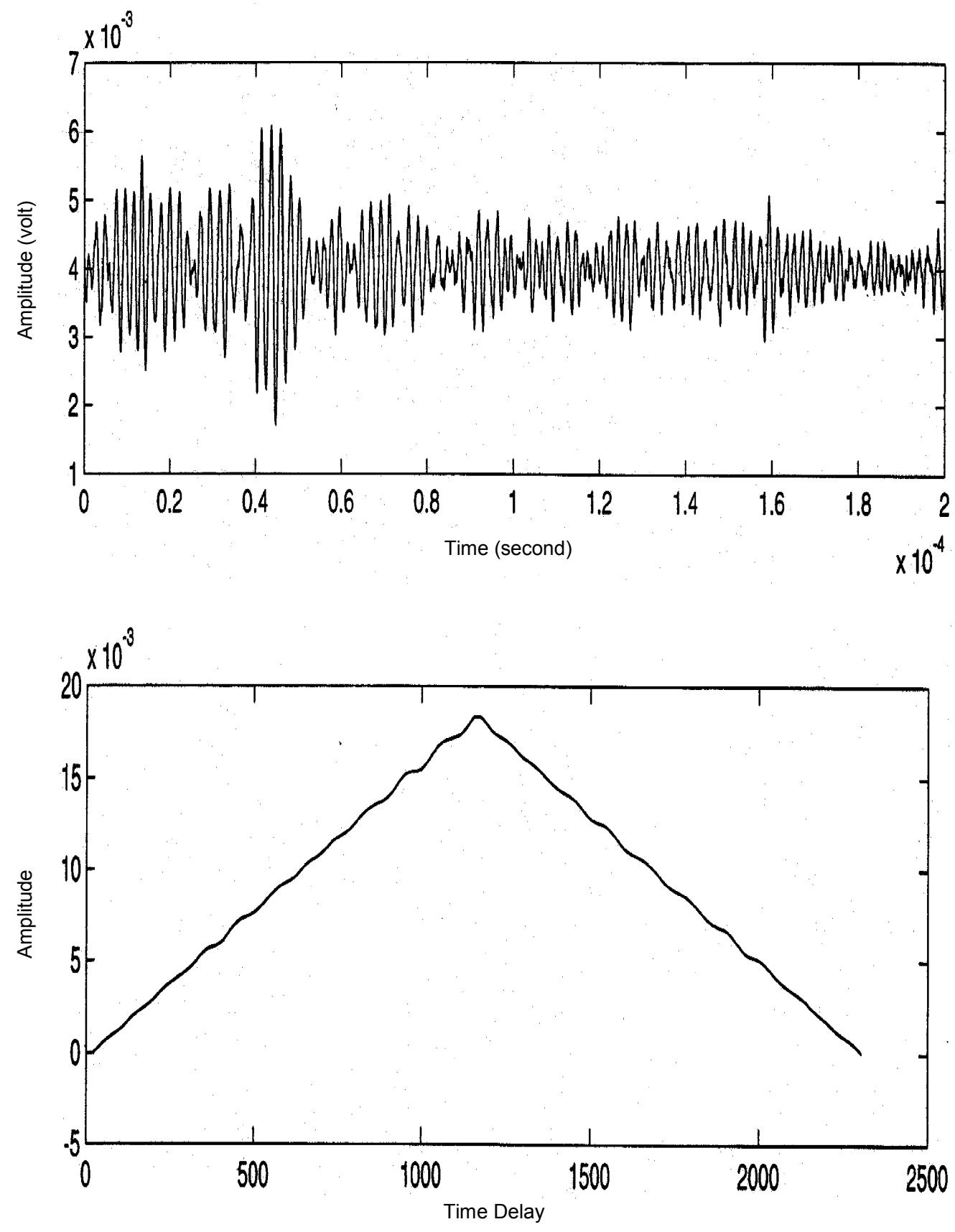

(a) Pressure: 14.41 psia

Figure A.13. Example of $500-\mathrm{kHz}$ ultrasonic waveforms measured in Natural Gas \#2 and associated cross-correlation function. 

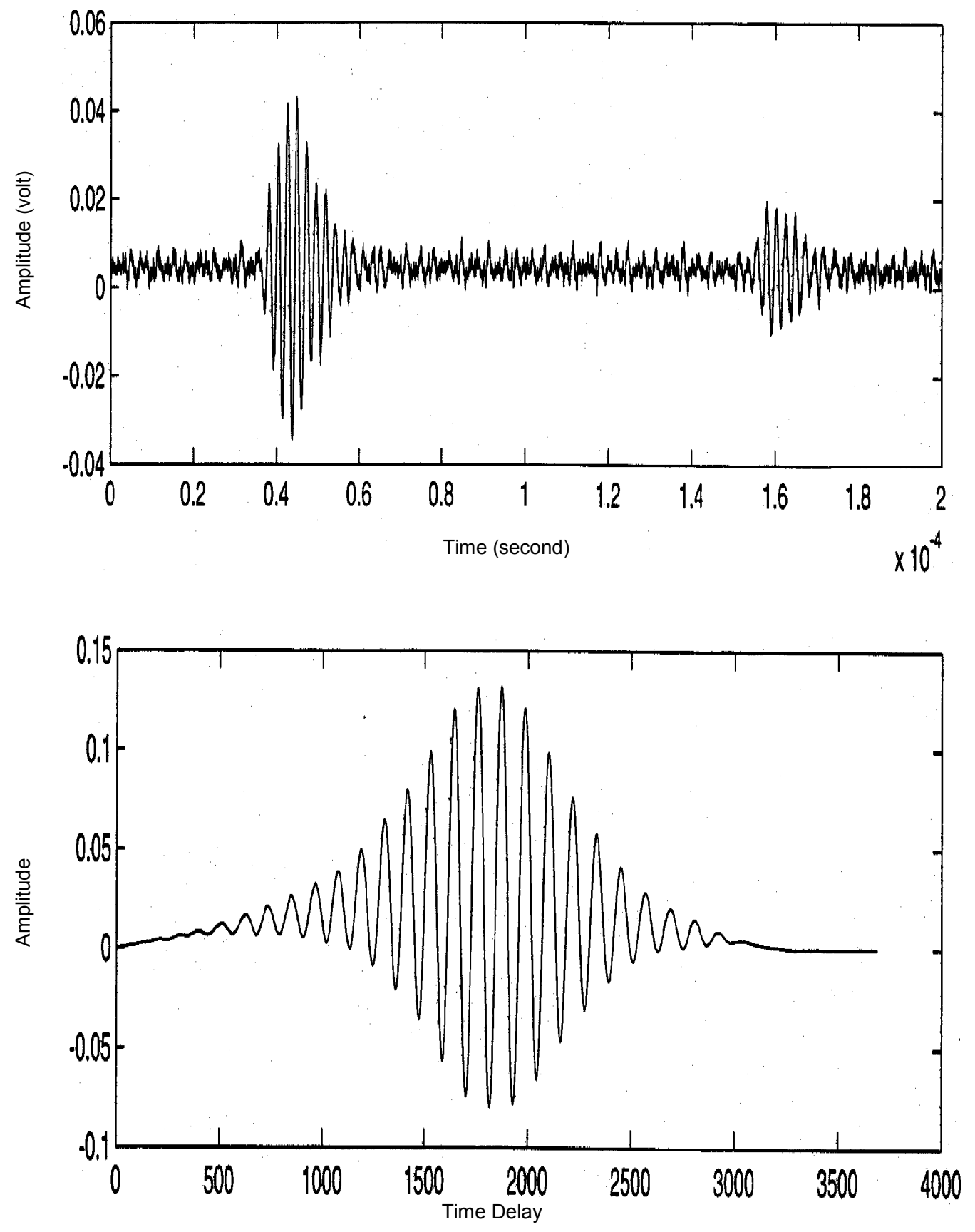

(b) Pressure: 29.66 psia

Figure A.13. Example of $500-\mathrm{kHz}$ ultrasonic waveforms measured in Natural Gas \#2 and associated cross-correlation function. (continued) 

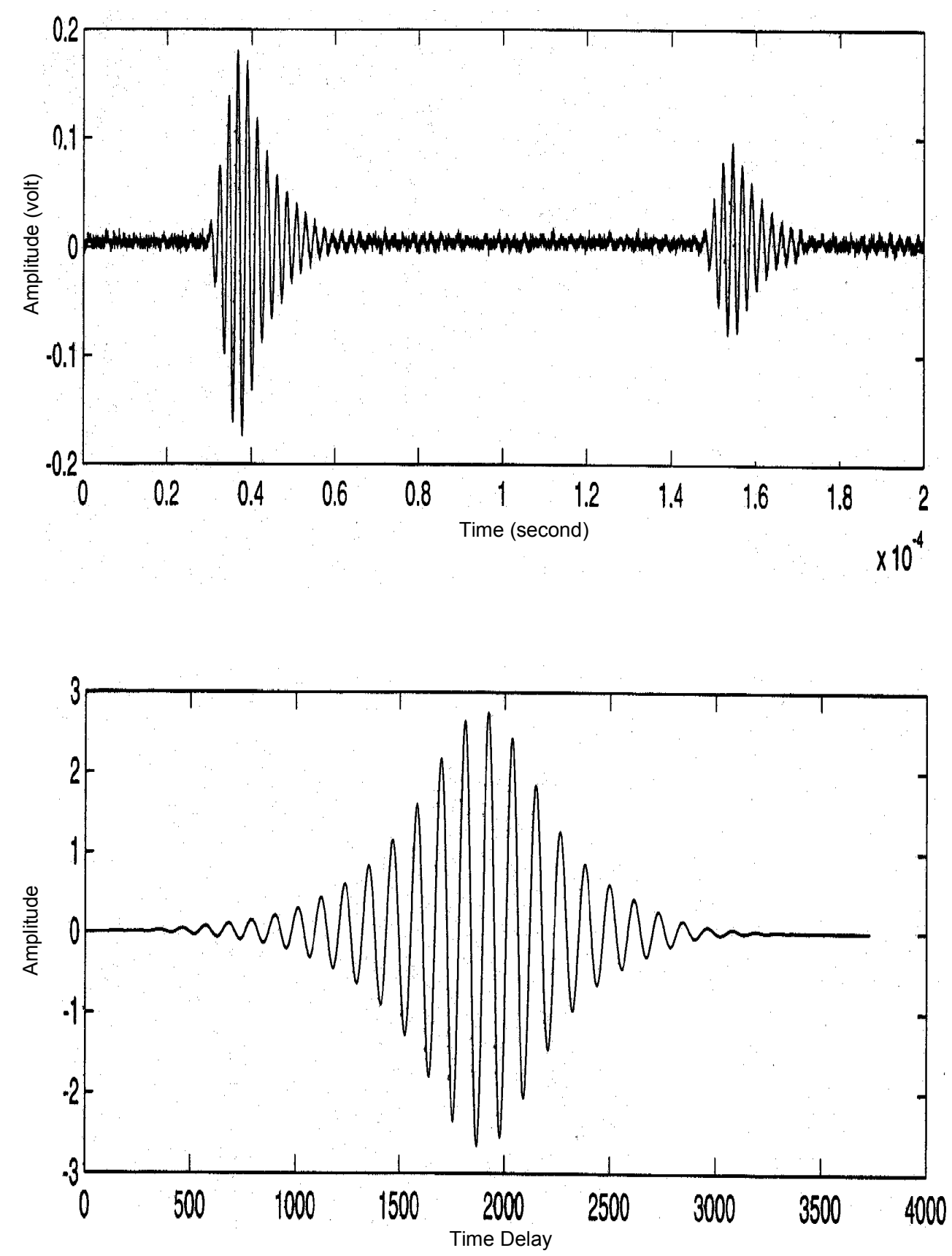

(c) Pressure: 47.54 psia

Figure A.13. Example of $500-\mathrm{kHz}$ ultrasonic waveforms measured in Natural Gas \#2 and associated cross-correlation function. (continued) 


\section{A.8 1000-kHz Transducer Tests}

Preliminary speed-of-sound measurements were performed at $1000 \mathrm{kHz}$ using the Ultran Model NCT-510 ultrasonic transducer installed in the test chamber with the near reflector face at a distance of $1.25 \mathrm{in}$. from the transducer. These tests indicated that ultrasonic absorption effects in Pure Nitrogen and in Natural Gas \#1 were excessive, resulting in signals of very low or negative signal-to-noise ratio. However, by operating below the $1000-\mathrm{kHz}$ resonance frequency, at a frequency of $750 \mathrm{kHz}$, usable signals were obtained in Natural Gas \#1 and Natural Gas \#3. Table A.4 lists the pressure and temperature test conditions and the differential time of flight derived from the $750-\mathrm{kHz}$ ultrasonic signal correlation analyses for reflection faces separated at 1.0024 in. apart.

Table A.4. Test data at $750 \mathrm{kHz}$ using Ultran Model NCT-510 ultrasonic transducer.

\begin{tabular}{|c|c|c|c|c|c|}
\hline \multicolumn{3}{|c|}{ Natural Gas \#1 } & \multicolumn{3}{|c|}{ Natural Gas \#3 } \\
\hline $\begin{array}{l}\text { Press } \\
\text { (psia) }\end{array}$ & $\begin{array}{c}\text { Temp } \\
\left({ }^{\circ} \mathrm{F}\right)\end{array}$ & $\begin{array}{c}\Delta t \\
(\mu s)\end{array}$ & $\begin{array}{l}\text { Press } \\
\text { (psia) }\end{array}$ & $\begin{array}{c}\text { Temp } \\
\left({ }^{\circ} \mathrm{F}\right)\end{array}$ & $\begin{array}{c}\Delta t \\
(\mu \mathbf{t})\end{array}$ \\
\hline 40.99 & 72.70 & 135.666 & 70.23 & 73.10 & 118.528 \\
\hline 50.57 & 72.70 & 135.806 & 75.30 & 73.10 & 118.548 \\
\hline 60.69 & 72.70 & 136.006 & 80.56 & 73.20 & 118.568 \\
\hline 71.19 & 72.80 & 136.166 & 85.26 & 73.00 & 118.608 \\
\hline 80.80 & 72.80 & 136.366 & 89.66 & 73.10 & 118.668 \\
\hline - & - & - & 94.94 & 73.10 & 118.728 \\
\hline - & - & - & 99.96 & 73.70 & 118.778 \\
\hline - & - & - & - & - & - \\
\hline $\mathrm{T}_{\text {avg }}$ & 72.740 & & $T_{\text {avg }}$ & 73.043 & \\
\hline
\end{tabular}

Speed of Sound in Natural Gas \#1: Figure A.14 shows the experimentally measured speed of sound versus pressure in Natural Gas \#1 at $750 \mathrm{kHz}$. Also shown in this figure is the calculated speed of sound adjusted to correspond to the average temperature of the ultrasonic test chamber. The measured speed of sound is lower than the calculated speed of sound by $-2.27 \mathrm{ft} / \mathrm{s}$ at the 60-psia test pressure, corresponding to an offset of $-0.184 \%$. The pressure-dependent slope of the measured speed of sound is essentially the same as that of the calculated curve at pressures of $40 \mathrm{psia}$ and higher. Because the transducer was operating below its natural resonance frequency, the received ultrasonic signals at gas pressures below 40 psia were too noisy to permit useful correlation analysis. The magnitude of the offset in speed of sound $(-2.27 \mathrm{ft} / \mathrm{s})$ is only about $5 \%$ of that which would be caused by a skipped cycle in the differential time of flight from the correlated waveforms. A potential source of this offset error may be associated with the fact that the reflector faces are well inside the near field of the $750-\mathrm{kHz}$ transducer where diffraction effects and wavefront phase differences between the two reflections could possibly introduce a differential time delay to perturb the measured speed of sound. 


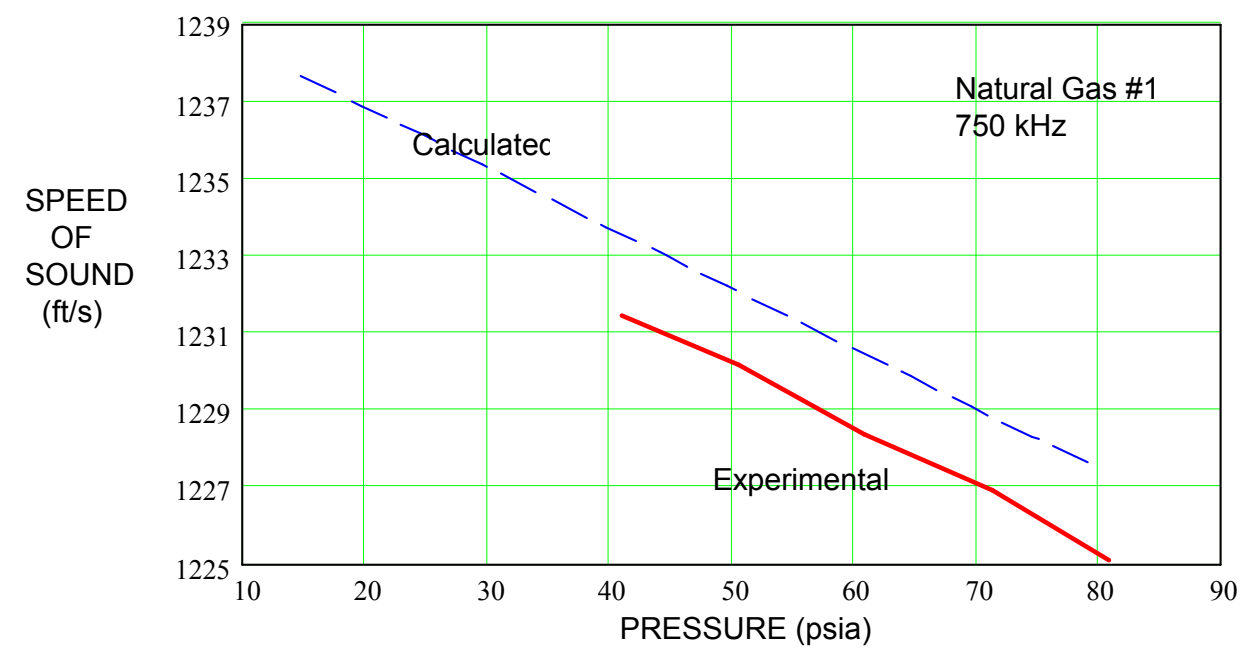

Figure A.14. Speed of sound measured at $750 \mathrm{kHz}$ in Natural Gas \#1.

Figure A.15 shows examples of the $750-\mathrm{kHz}$ ultrasonic waveforms recorded at medium and high test pressures in Natural Gas \#1. Also shown are the corresponding correlation functions derived from these waveforms. Not indicated to scale in these illustrations are the relative amplitudes of the waveforms; however, the relatively low signal-to-noise ratios of both reflection signals are readily evident. The received pulses at $750 \mathrm{kHz}$ have a relatively poor signal-to-noise ratio for three reasons:

(1) viscous absorption effects at frequencies above about $500 \mathrm{kHz}$ in the methane-dominant gas are significant;

(2) the ultrasonic pulse excitation signal at $750 \mathrm{kHz}$ is substantially below the resonance frequency of the $1000-\mathrm{kHz}$ transducer where transducer sensitivity is relatively low; and

(3) the transducer acoustic coupling efficiency is directly proportional to the gas density (pressure) and, in combination with low off-resonance sensitivity, makes the transducer unusable below about 70 psia.

Speed of Sound in Natural Gas \#3: Figure A.16 shows the experimentally measured speed of sound versus pressure in Natural Gas \#3 at $750 \mathrm{kHz}$. Also shown in this figure is the calculated speed of sound adjusted to correspond to the average temperature of the ultrasonic test chamber. The measured speed of sound is higher than the calculated speed of sound by $+0.89 \mathrm{ft} / \mathrm{s}$ at the 85-psia test pressure, corresponding to an offset of $+0.07 \%$. Measurements using this transducer were not practical at gas pressures below 70 psia because of poor signal-to-noise ratio at $750 \mathrm{kHz}$. 

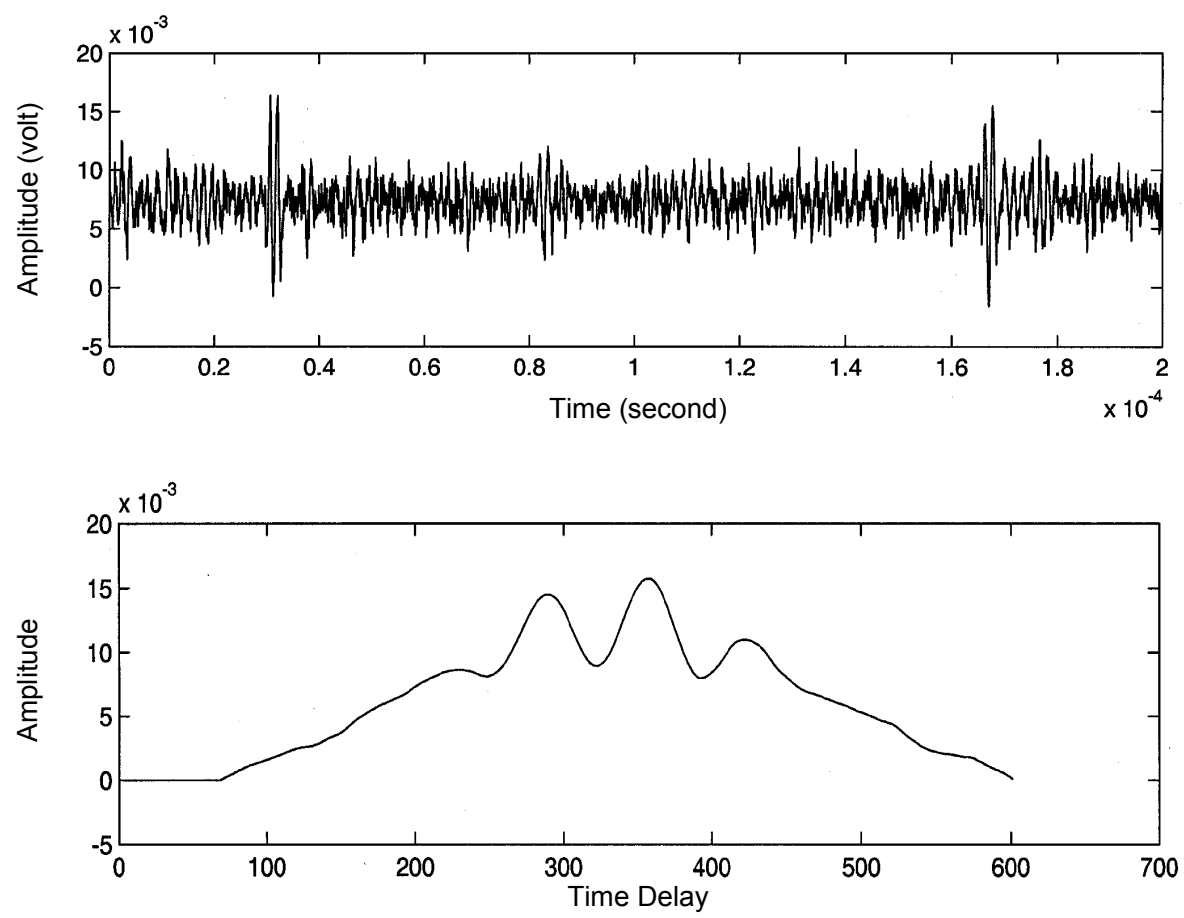

(a) Pressure: 40.99 psia
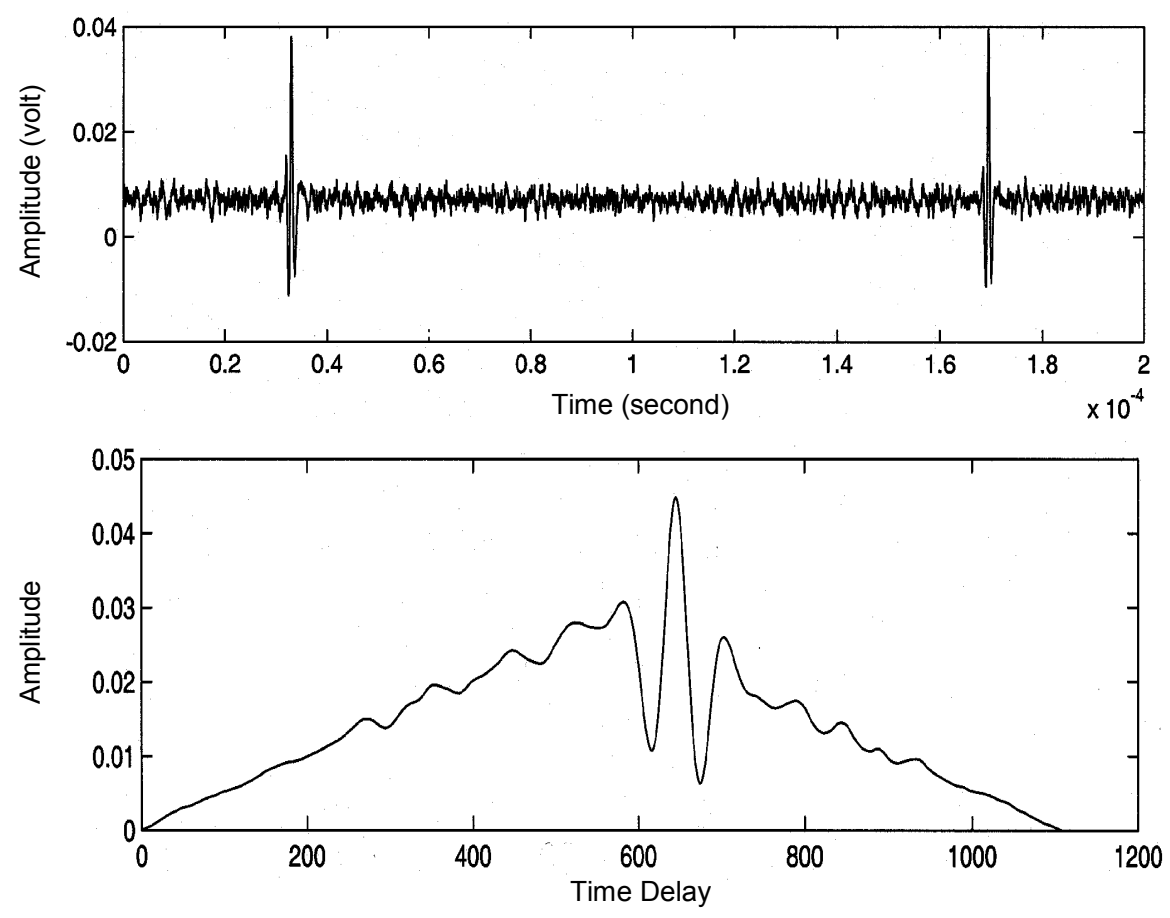

(b) Pressure: 80.79 psia

Figure A.15. Example of $750-\mathrm{kHz}$ ultrasonic waveforms measured in Natural Gas \#1 and associated cross-correlation function. 


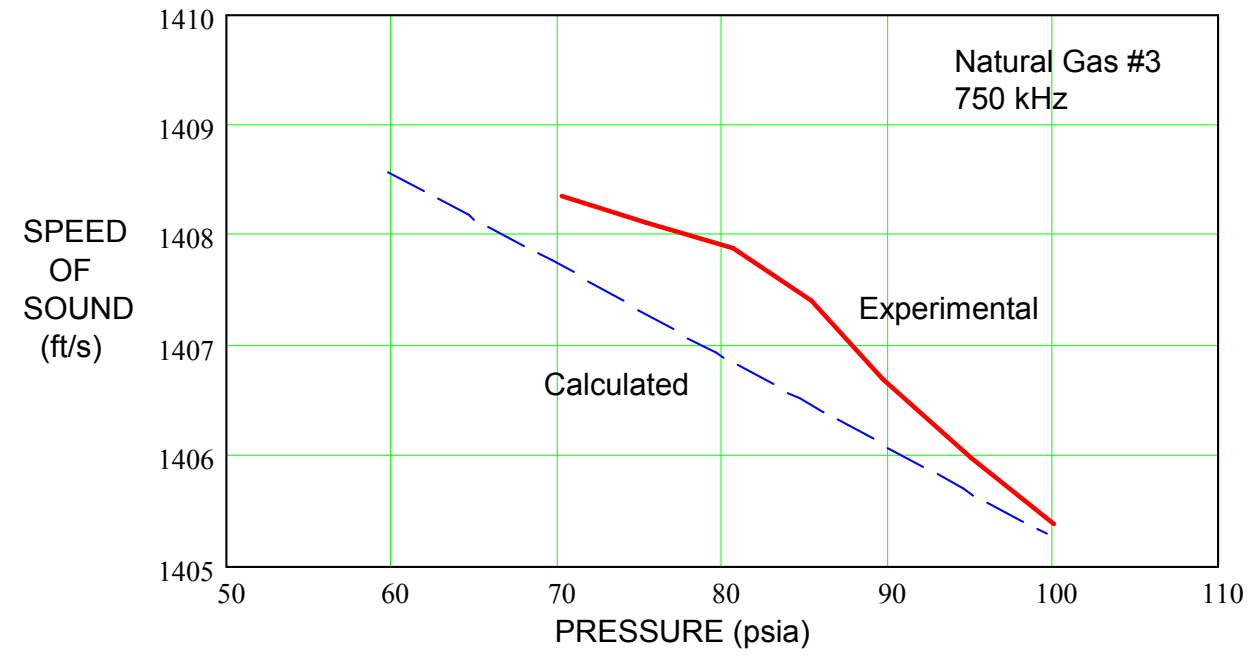

Figure A.16. Speed of sound measured at $750 \mathrm{kHz}$ in Natural Gas \#3.

Figure A.17 shows examples of the $750-\mathrm{kHz}$ ultrasonic waveforms recorded at medium and high test pressures in Natural Gas \#3. Also shown are the corresponding correlation functions derived from these waveforms. Not indicated to scale in these illustrations are the relative amplitudes of the waveforms; however, the relatively low signal-to-noise ratios of both reflected signals are readily evident for the same reasons explained in reference to the waveforms in Figure A.15. 

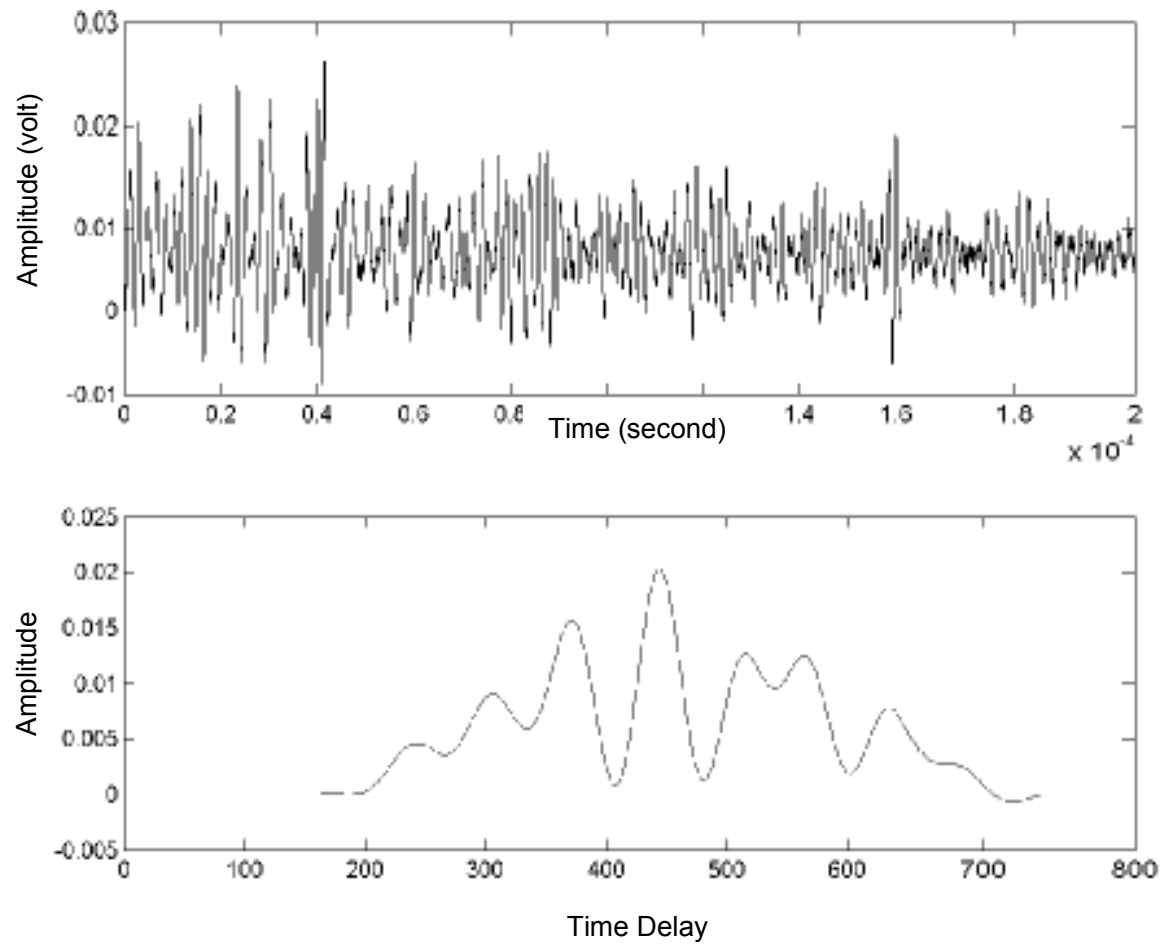

(a) Pressure: 59.58 psia
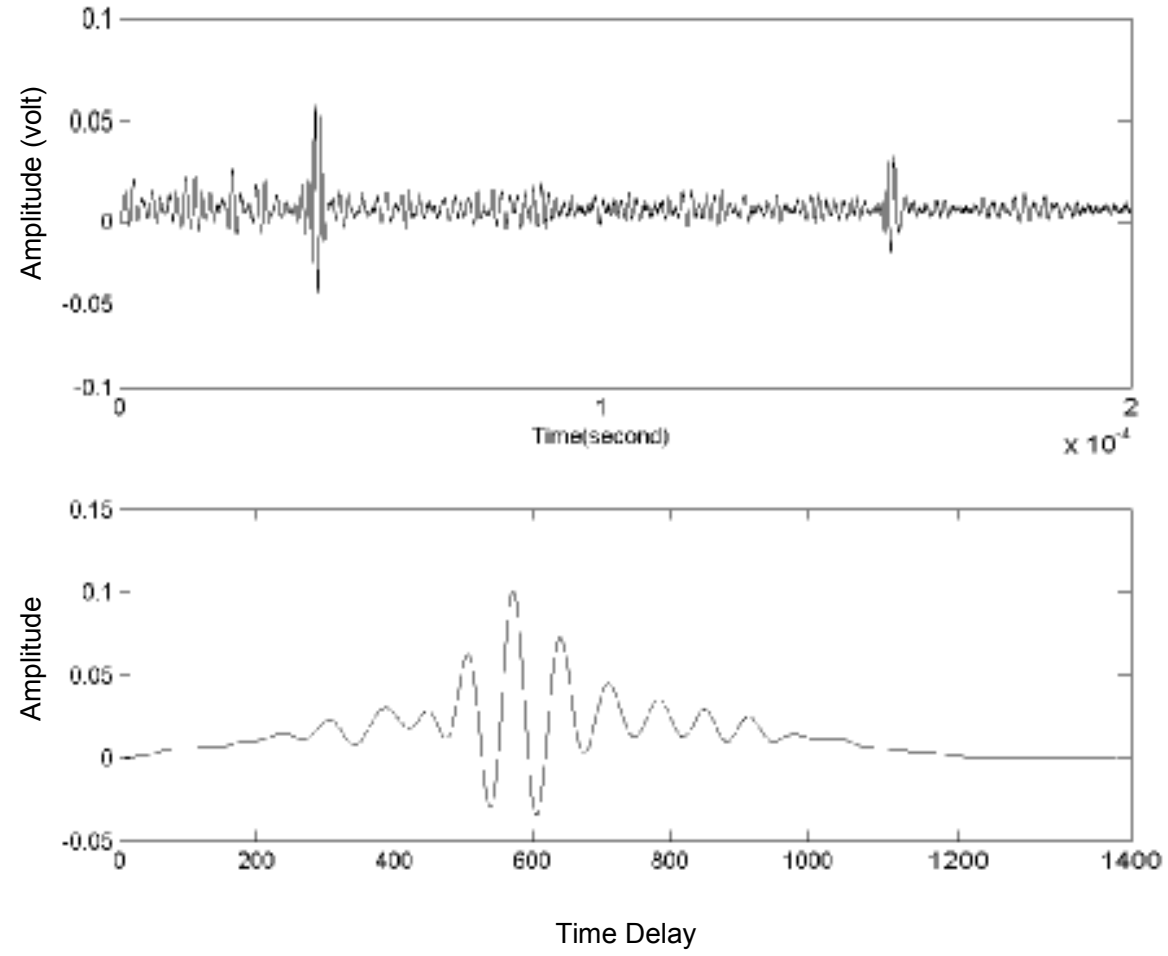

(b) Pressure: 99.50 psia

Figure A-17. Example of $750-k H z$ ultrasonic waveforms measured in Natural Gas \#3 and associated cross-correlation function. 


\section{A.9 279-kHz Transducer Tests}

Speed-of-sound measurements were performed at $279 \mathrm{kHz}$ using the Etalon Model CIA3525-SB ultrasonic transducer installed in the test chamber with the near reflector face at a distance of 3.25 in. from the transducer face. Three test gases were used: Pure Nitrogen, Natural Gas \#1, and Natural Gas \#3. Table A.5 lists the pressure and temperature test conditions and the differential time of flight derived from the $279-\mathrm{kHz}$ ultrasonic signal correlation analysis for reflection faces separated $1.0016 \mathrm{in}$. apart.

Speed of Sound in Nitrogen: Figure A.18 shows the experimentally measured speed of sound versus pressure in nitrogen at $279 \mathrm{kHz}$. Also shown in this figure is the calculated speed of sound adjusted to correspond to the average temperature of the ultrasonic test chamber. The measured speed of sound is higher than the calculated speed of sound by $0.79 \mathrm{ft} / \mathrm{s}$ at the 40 -psia test pressure, corresponding to an offset of $+0.069 \%$. The pressure-dependent slope of the measured speed of sound is, on average, the same as the slope of the calculated speed of sound over the range of test pressures. Variations in measured speed of sound relative to the mean slope are $\pm 0.31 \mathrm{ft} / \mathrm{s}$ (at $40 \mathrm{psia}$ ) or less.

Table A.5. Test data at $279 \mathrm{kHz}$ using Etalon Model CIA-3525-SB ultrasonic transducer.

\begin{tabular}{|c|c|c|c|c|c|c|c|c|}
\hline \multicolumn{3}{|c|}{ Pure Nitrogen } & \multicolumn{3}{|c|}{ Natural Gas \#1 } & \multicolumn{3}{|c|}{ Natural Gas \#3 } \\
\hline $\begin{array}{l}\text { Press } \\
\text { (psia) }\end{array}$ & $\begin{array}{c}\text { Temp } \\
\left({ }^{\circ} \mathrm{F}\right)\end{array}$ & $\begin{array}{c}\Delta t \\
(\mu s e c)\end{array}$ & $\begin{array}{l}\text { Press } \\
\text { (psia) }\end{array}$ & $\begin{array}{c}\text { Temp } \\
\left({ }^{\circ} \mathrm{F}\right)\end{array}$ & $\begin{array}{c}\Delta t \\
(\mu s e c)\end{array}$ & $\begin{array}{l}\text { Press } \\
\text { (psia) }\end{array}$ & $\begin{array}{c}\text { Temp } \\
\left({ }^{\circ} \mathrm{F}\right)\end{array}$ & $\underset{(\mu s e c)}{\Delta t}$ \\
\hline 15.257 & 71.70 & 145.186 & 15.055 & 72.40 & 135.107 & 20.430 & 73.30 & 118.188 \\
\hline 20.224 & 71.60 & 145.186 & 20.082 & 72.40 & 135.147 & 30.140 & 73.30 & 118.228 \\
\hline 30.294 & 71.60 & 145.106 & 25.076 & 72.30 & 135.187 & 40.031 & 73.40 & 118.308 \\
\hline 40.196 & 71.80 & 145.026 & 30.089 & 72.30 & 135.267 & 50.640 & 73.40 & 118.348 \\
\hline 50.232 & 71.80 & 145.026 & 35.136 & 72.30 & 135.347 & 60.262 & 73.50 & 118.428 \\
\hline 60.296 & 71.80 & 144.946 & 40.086 & 72.30 & 135.427 & 70.775 & 73.50 & 118.508 \\
\hline 70.497 & 71.80 & 144.906 & 44.905 & 72.30 & 135.506 & 80.014 & 73.50 & 118.548 \\
\hline 80.136 & 71.80 & 144.866 & 49.803 & 72.20 & 135.586 & 90.468 & 73.20 & 118.668 \\
\hline 89.964 & 71.5 & 144.866 & 55.110 & 72.20 & 135.666 & 95.031 & 73.20 & 118.668 \\
\hline 94.722 & 71.5 & 144.866 & 60.382 & 72.30 & 135.786 & 100.334 & 73.20 & 118.708 \\
\hline- & - & - & 65.080 & 72.20 & 135.826 & - & - & - \\
\hline - & - & - & 69.850 & 72.30 & 135.906 & - & - & - \\
\hline- & - & - & 74.426 & 72.20 & 136.026 & - & - & - \\
\hline $\mathrm{T}_{\text {avg }}$ & 71.673 & & $T_{\text {avg }}$ & 72.285 & & $T_{\text {avg }}$ & 73.355 & \\
\hline
\end{tabular}




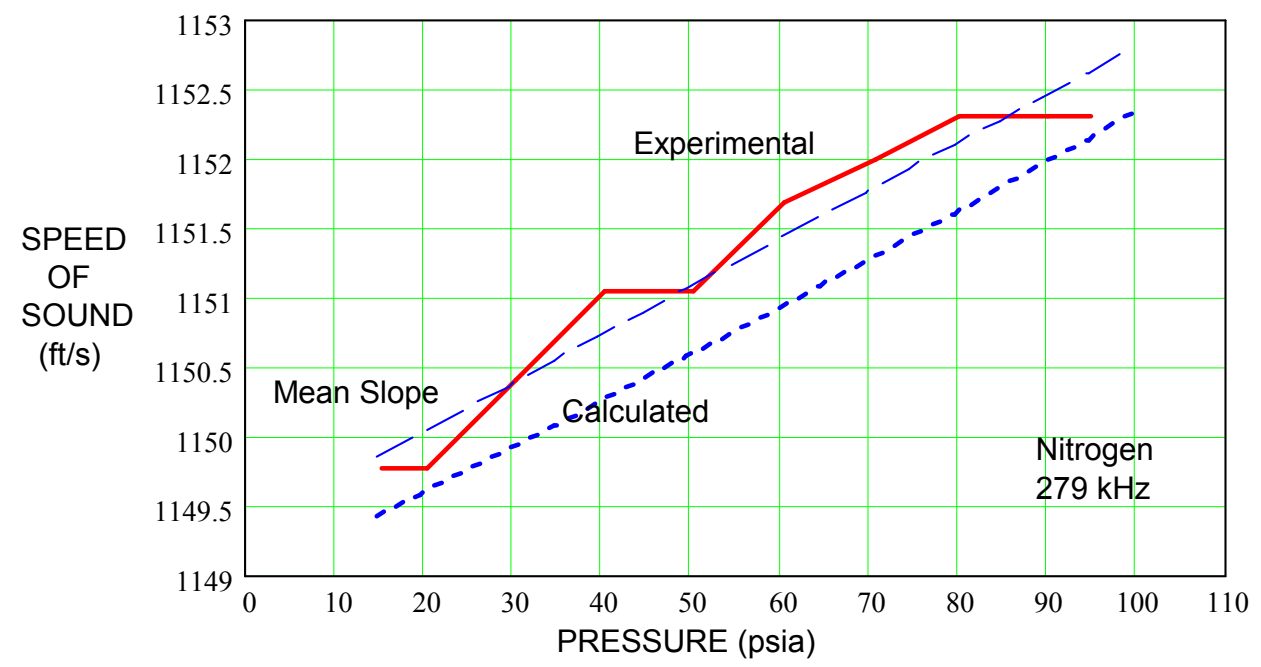

Figure A.18. Speed of sound measured at $279 \mathrm{kHz}$ in nitrogen.

Figure A.19 shows examples of the $279-\mathrm{kHz}$ ultrasonic waveforms recorded at low, medium, and high test pressures in nitrogen. Also shown are the corresponding correlation functions derived from these waveforms. Not indicated to scale in these illustrations are the relative amplitudes of the waveforms, which vary in approximate direct proportion with the test pressure.

The time base showing the two reflected pulses in Figure A.19 is shifted from its origin by an amount of $-357.6 \mu \mathrm{s}$ to clearly illustrate the pulse waveforms. These pulse waveforms have a signal-to-noise ratio ranging from about 15 to 1 at the low test pressure of 14.27 psia, to about 45 to 1 at the high test pressure of 94.72 psia. This increase with pressure is consistent with the increase in transducer acoustic coupling efficiency for increasing gas density. However, the relatively high value of absolute viscosity of nitrogen $\left(\mathrm{N}_{2}: 170.2 \mu\right.$ poise at STP) compared with the hydrocarbon gases tested (NG \#1: $107.9 \mu$ poise; NG \#3: $106.5 \mu$ poise) significantly reduces the received ultrasonic signals in nitrogen. The two pulse waveforms are closely matched in shape and have a rise time (to peak) of about three oscillatory periods $(\sim 10 \mu s)$ and a decay time of about eight periods $(\sim 29 \mu \mathrm{s})$. These waveforms are good approximations of the impulse response of the transducer, i.e., the output waveform generated when the transducer is uniformly excited over its complete frequency spectrum, since the applied excitation voltage was a broad bandwidth partial sine wave pulse ( $\sim 2 \mu$ s time duration). The combined number of cycles in the waveform determines the number of oscillatory correlation peaks in the correlation function $(\sim 20$ significant peaks). The maximum positive peak is indicative of the statistical 'best fit' time lag for coherence of the two pulses. The noise level present in the waveform trace is essentially absent from the correlation function because it is of a different (incoherent) origin than the ultrasonic pulses. For the particular pulses shown, the correlation peaks adjacent to the maximum coherence lobe are lower in amplitude by only about $12 \%$. This difference proved to be adequate throughout the bench tests for automatically selecting the maximum coherence value corresponding to the correct time delay between the two pulses. Nevertheless, a more distinct coherence lobe, attainable with a wider bandwidth transducer, would provide a greater margin of reliability in avoiding the possibility of correlation cycle skipping errors under more extreme operating conditions. 

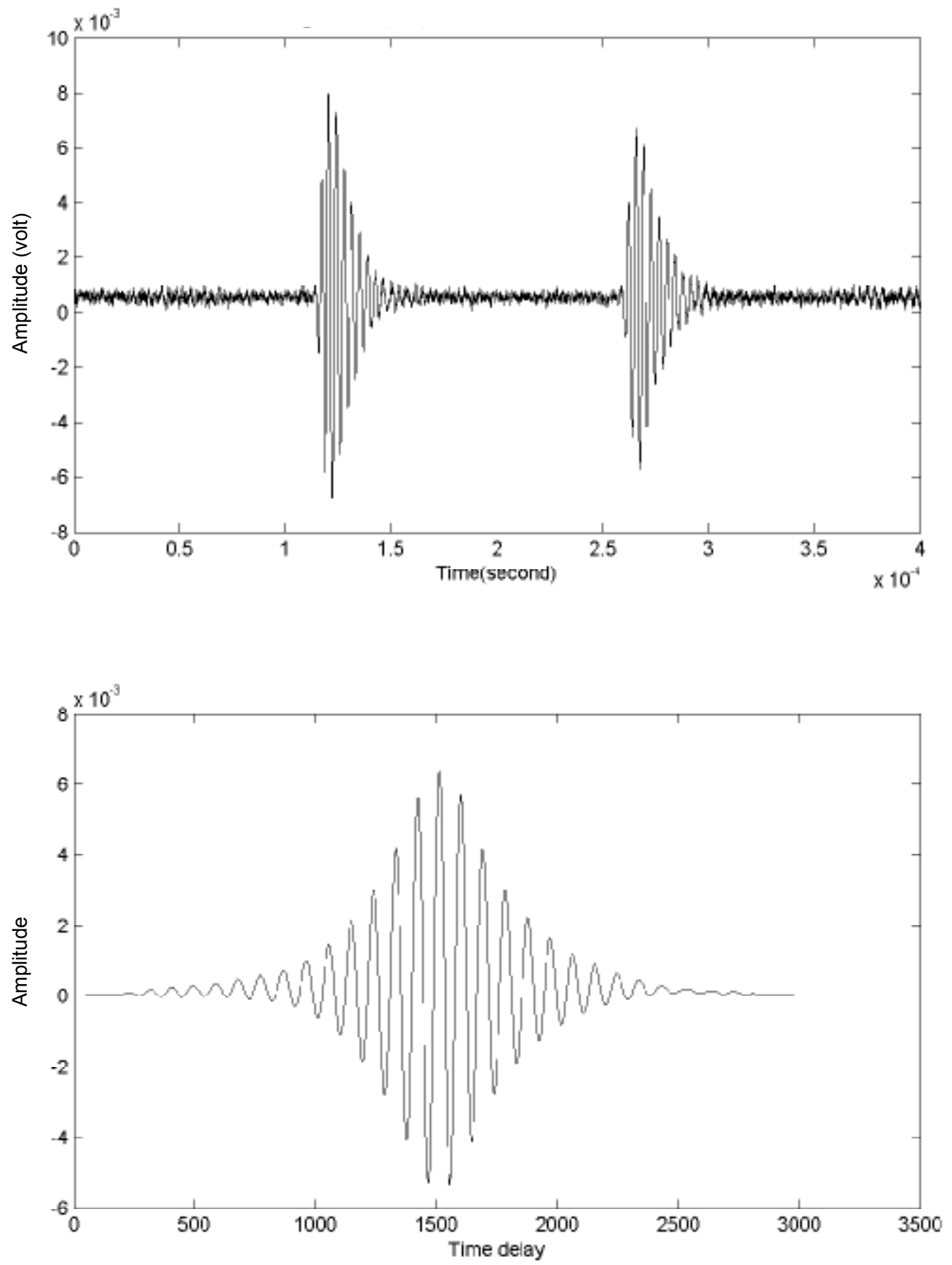

(a) Pressure: 14.27 psia

Figure A.19. Example of 279-kHz ultrasonic waveforms measured in nitrogen and associated cross-correlation function. 

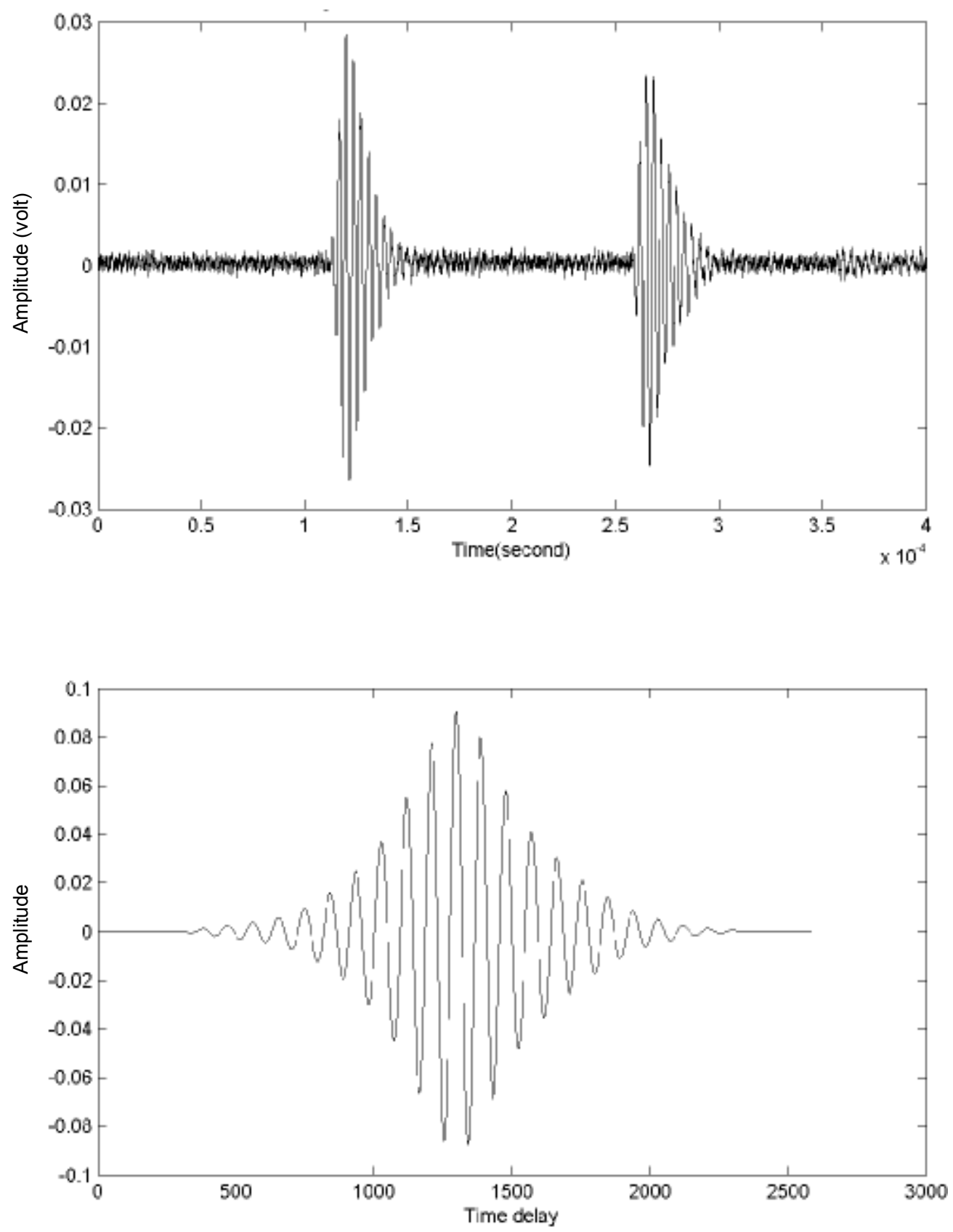

(b) Pressure: 40.77 psia

Figure A.19. Example of 279-kHz ultrasonic waveforms measured in nitrogen and associated cross-correlation function (continued). 

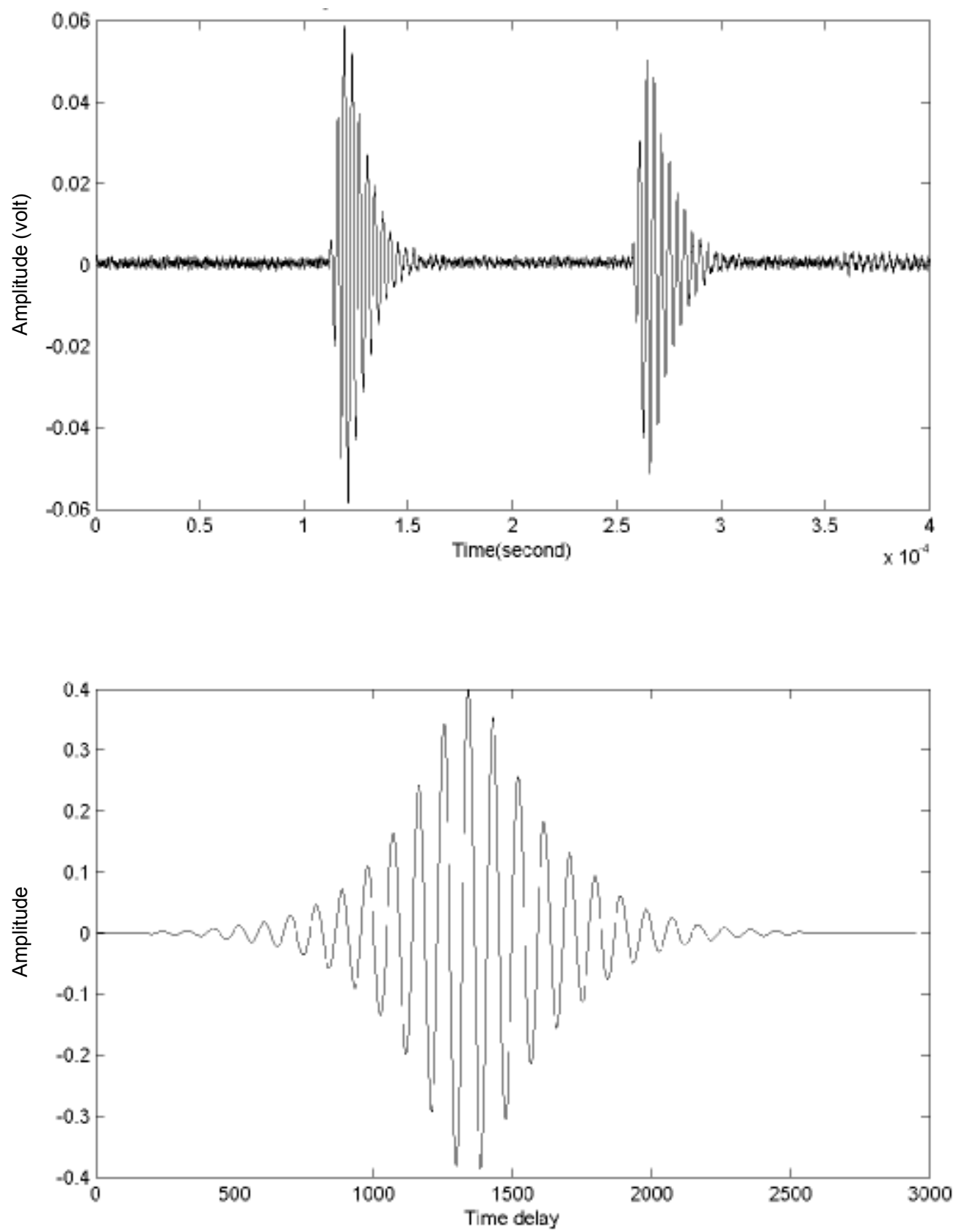

(c) Pressure: 70.93 psia

Figure A.19. Example of 279-kHz ultrasonic waveforms measured in nitrogen and associated cross-correlation function (continued). 
Speed of Sound in Natural Gas \#1: Figure A.20 shows the experimentally measured speed of sound versus pressure in Natural Gas \#1 at $279 \mathrm{kHz}$. Also shown in this figure is the calculated speed of sound adjusted to correspond to the average temperature of the ultrasonic test chamber. The measured speed of sound is lower than the calculated speed of sound by $-1.69 \mathrm{ft} / \mathrm{s}$ at the 40-psia test pressure, corresponding to an offset of $-0.137 \%$. The pressure-dependent slope of the measured speed of sound is essentially the same as that of the calculated curve over the pressure range tested. Variations in speed of sound away from the general trend indicated by the experimental data are negligible at test pressures above about 25 psia.

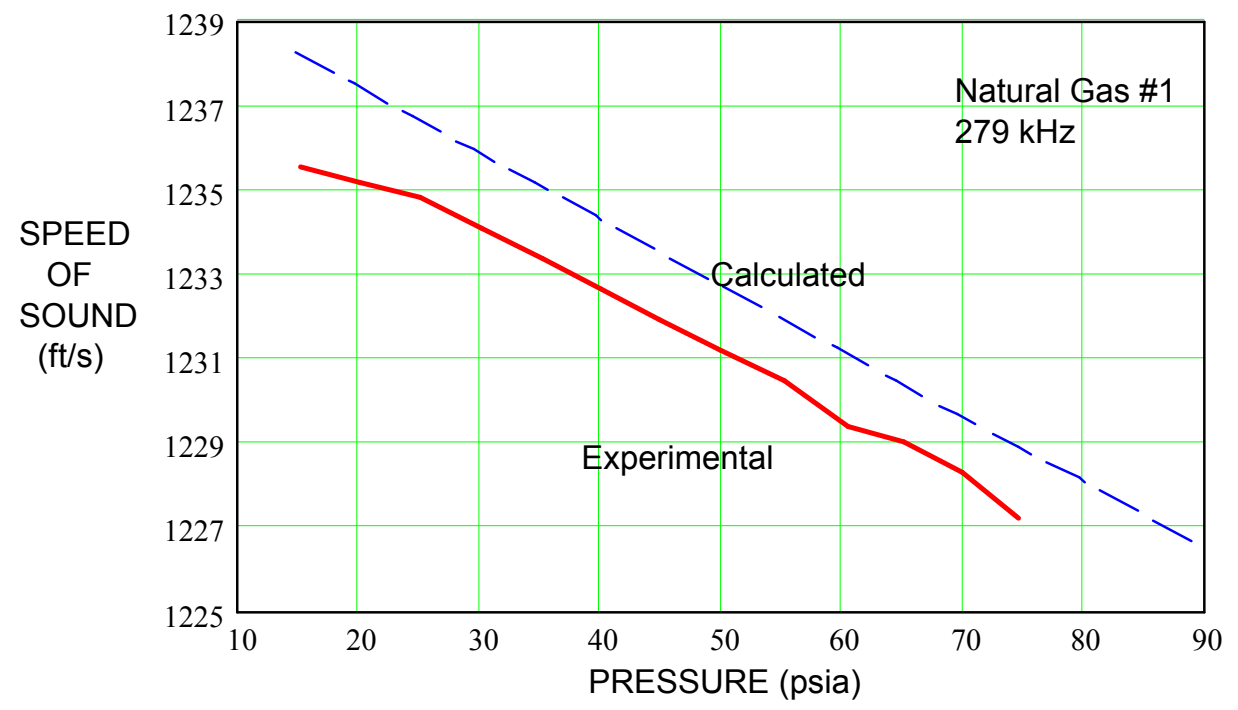

Figure A.20. Speed of sound measured at $279 \mathrm{kHz}$ in Natural Gas \#1.

Figure A.21 shows examples of the $279-\mathrm{kHz}$ ultrasonic waveforms recorded at low, medium, and high test pressures in Natural Gas \#1. Also shown are the corresponding correlation functions derived from these waveforms. Not indicated to scale in these illustrations are the relative amplitudes of the waveforms, which vary in approximate direct proportion with the test pressure.

The time base showing the two reflected pulses in Figure A.21 is shifted from its origin by an amount of $-357.6 \mu$ s to clearly illustrate the pulse waveforms. These pulse waveforms have a signal-to-noise ratio ranging from about 30 to 1 at the low test pressure of 15.06 psia, to about 70 to 1 at the high test pressure of 74.43 psia, characteristic of the lower attenuation in Natural Gas \#3 compared with that of nitrogen. The impulse response of the transducer, as noted from the pulse envelope shape and number of oscillatory cycles ( $\sim 10$ cycles), is identical to that observed in nitrogen. In this test, the correlation peaks adjacent to the maximum coherence lobe are lower in amplitude by about $15 \%$. This difference proved to be adequate throughout the bench tests for automatically selecting the maximum coherence value corresponding to the correct time delay between the two pulses. 

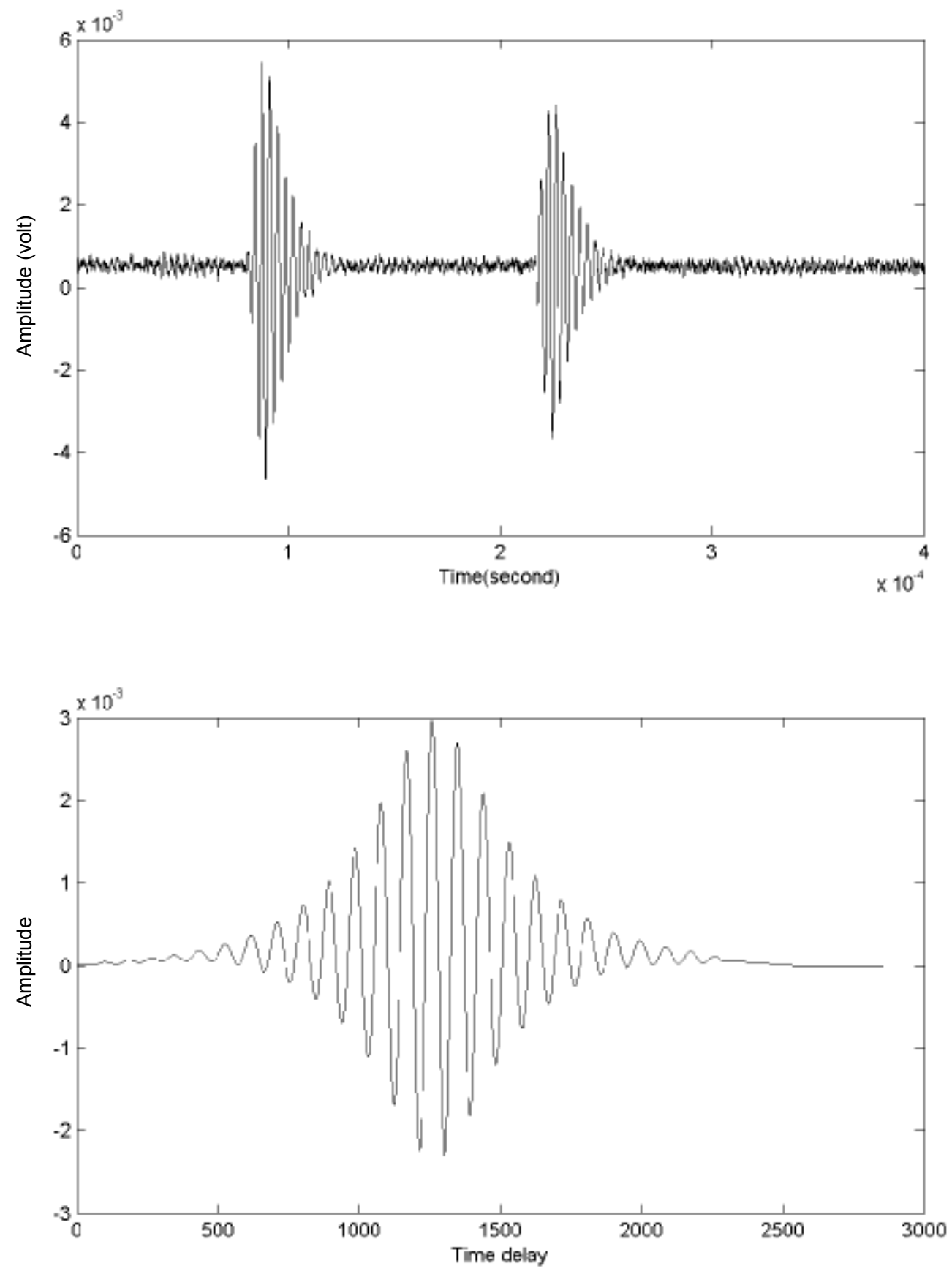

(a) Pressure: 14.38 psia

Figure A.21. Example of 279-kHz ultrasonic waveforms measured in Natural Gas \#1 and associated cross-correlation function. 

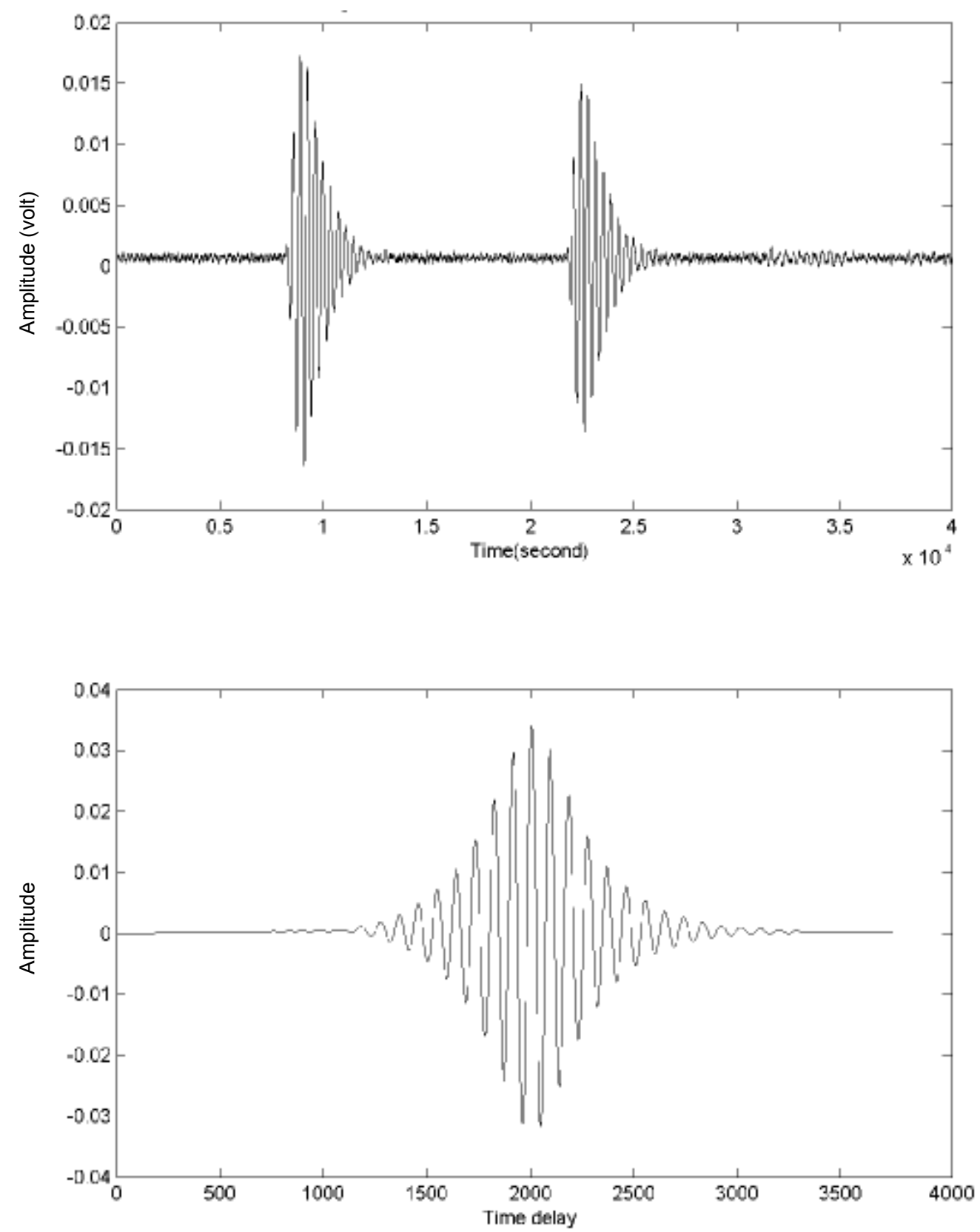

(b) Pressure: 40.39 psia

Figure A.21. Example of 279-kHz ultrasonic waveforms measured in Natural Gas \#1 and associated cross-correlation function (continued). 

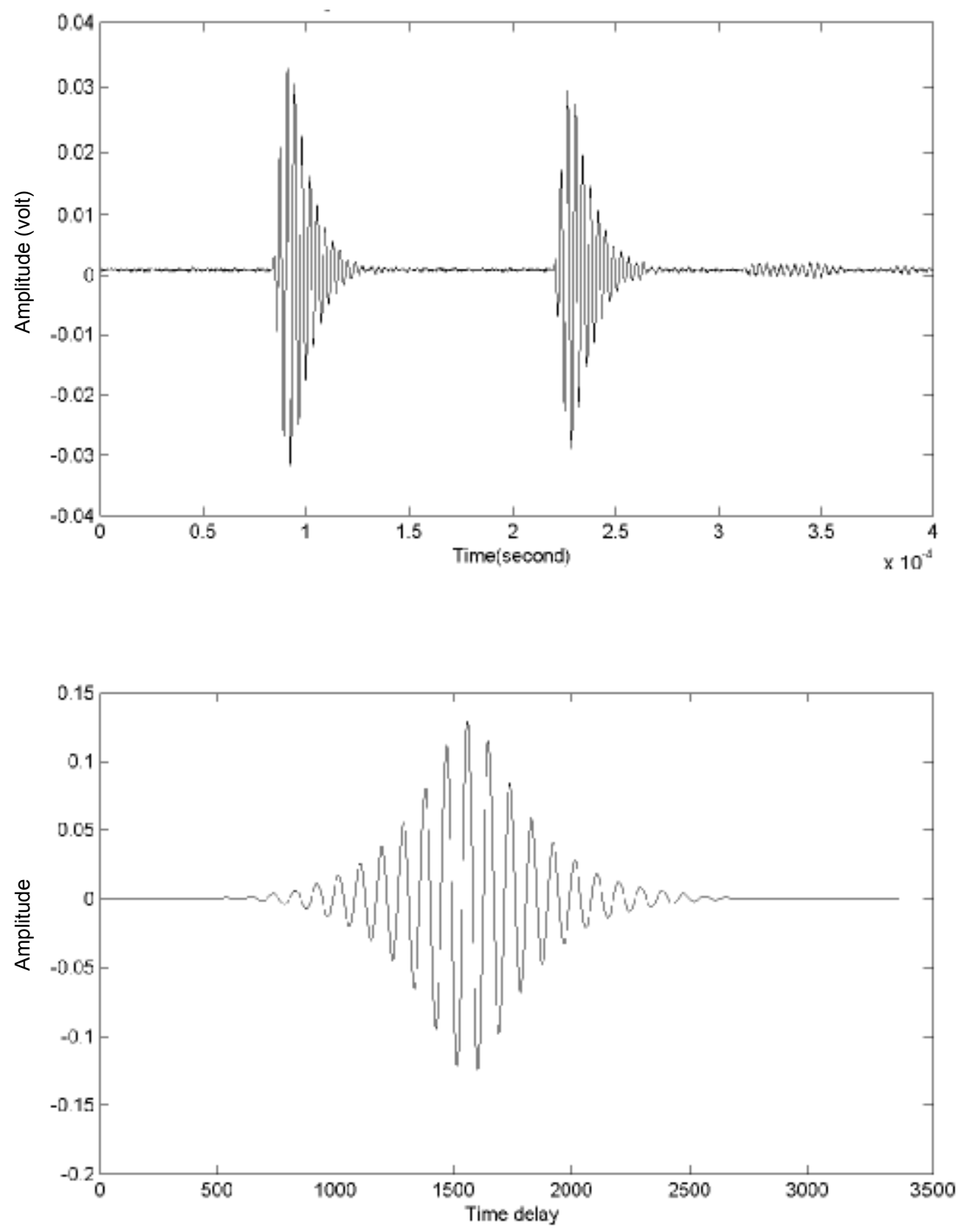

(c) Pressure: 69.44 psia

Figure A.21. Example of 279-kHz ultrasonic waveforms measured in Natural Gas \#1 and associated cross-correlation function (continued). 
Speed of Sound in Natural Gas \#3: Figure A.22 shows the experimentally measured speed of sound versus pressure in Natural Gas \#3 at $279 \mathrm{kHz}$. Also shown in this figure is the calculated speed of sound adjusted to correspond to the average temperature of the ultrasonic test chamber. The measured speed of sound is higher than the calculated speed of sound by $+0.31 \mathrm{ft} / \mathrm{s}$ at the 40 psia test pressure, corresponding to an offset of $+0.022 \%$. The slope of the measured speed of sound has approximately the same pressure dependence as that of the calculated values but tends to increase slightly with increasing pressure. The magnitude of the offset in speed of sound $(+0.31 \mathrm{ft} / \mathrm{s})$ is essentially within the practical resolving power of the experimental instrumentation system based on the digital sampling rate and related time resolution capabilities inherent in the time-of-flight correlation analysis.

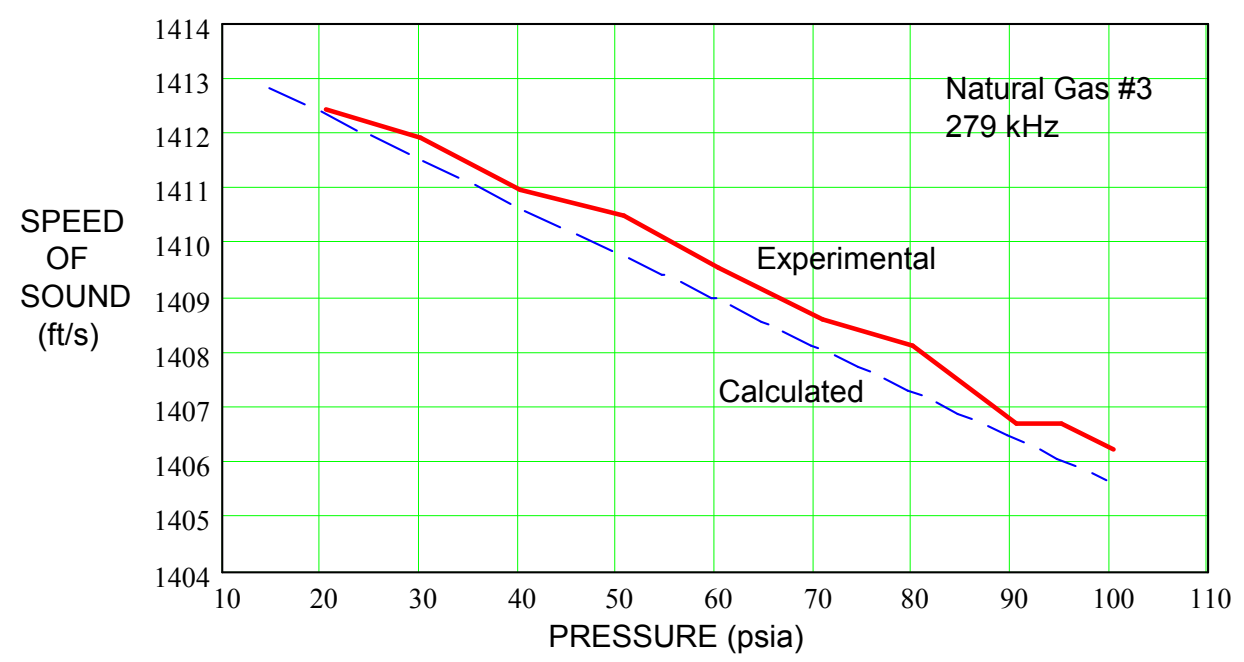

Figure A.22. Speed of sound measured at $279 \mathrm{kHz}$ in Natural Gas \#3.

Figure A.23 shows examples of the $279-\mathrm{kHz}$ ultrasonic waveforms recorded at low, medium, and high test pressures in Natural Gas \#3. Also shown are the corresponding correlation functions derived from these waveforms. Not indicated to scale in these illustrations are the relative amplitudes of the waveforms which vary in approximate direct proportion with the test pressure.

The time base showing the two reflected pulses in Figure A.23 is shifted from its origin by an amount of $-361.4 \mu$ s to clearly illustrate the pulse waveforms. These pulse waveforms have a signal-to-noise ratio ranging from about 30 to 1 at the low test pressure of 15.19 psia, to about 120 to 1 at the high test pressure of 95.03 psia, characteristic of the lower attenuation effects in Natural Gas \#3 compared with that of nitrogen. The impulse response of the transducer, as noted from the pulse envelope shape and number of oscillatory cycles $(\sim 10$ cycles), is identical to that observed in nitrogen. In this test, the correlation peaks adjacent to the maximum coherence lobe are lower in amplitude by about $14 \%$. This difference proved to be adequate throughout the bench tests for automatically selecting the maximum coherence value corresponding to the correct time delay between the two pulses. 

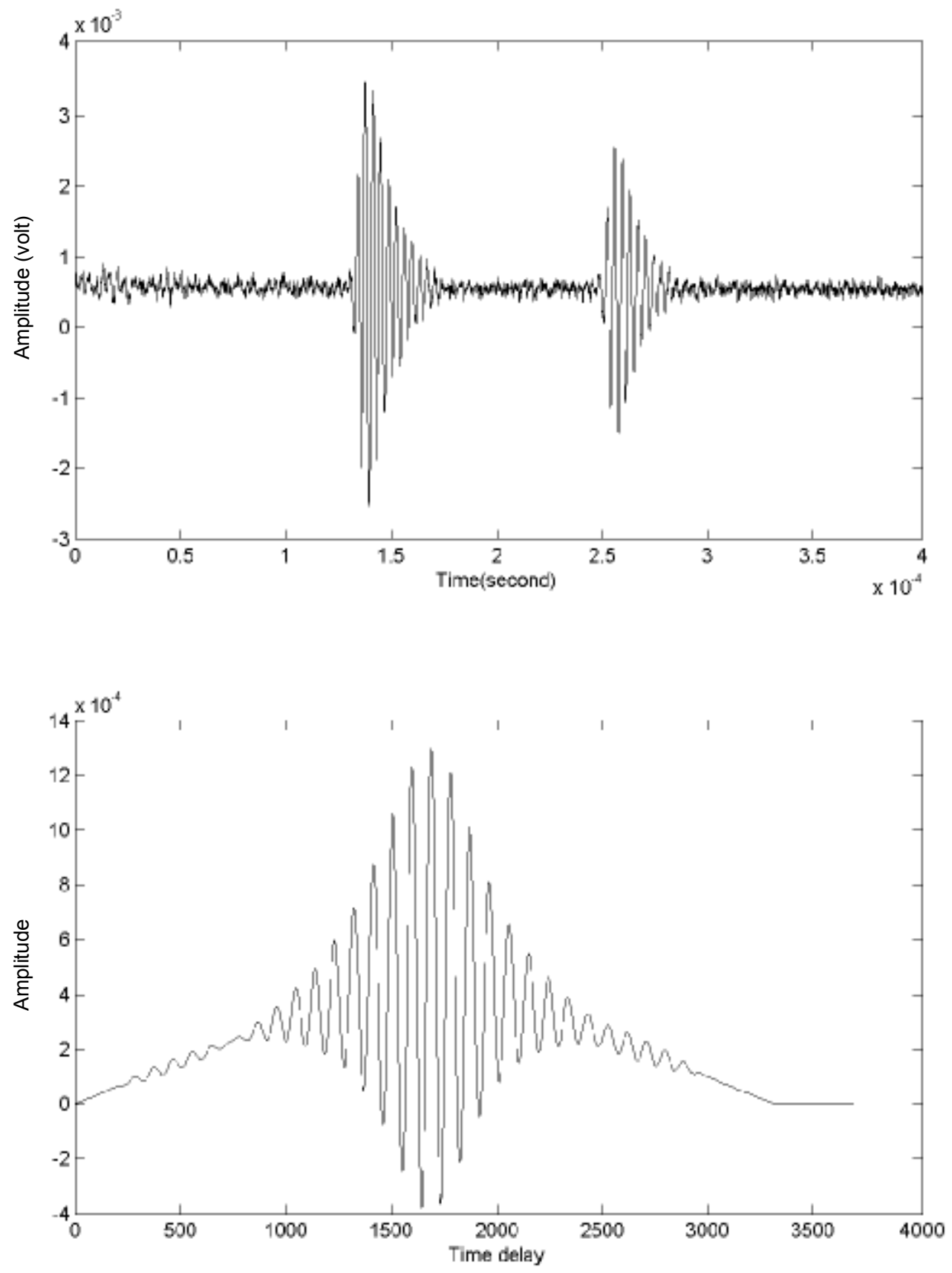

(a) Pressure: 14.41 psia

Figure A.23. Example of 279-kHz ultrasonic waveforms measured in Natural Gas \#3 and associated cross-correlation function. 

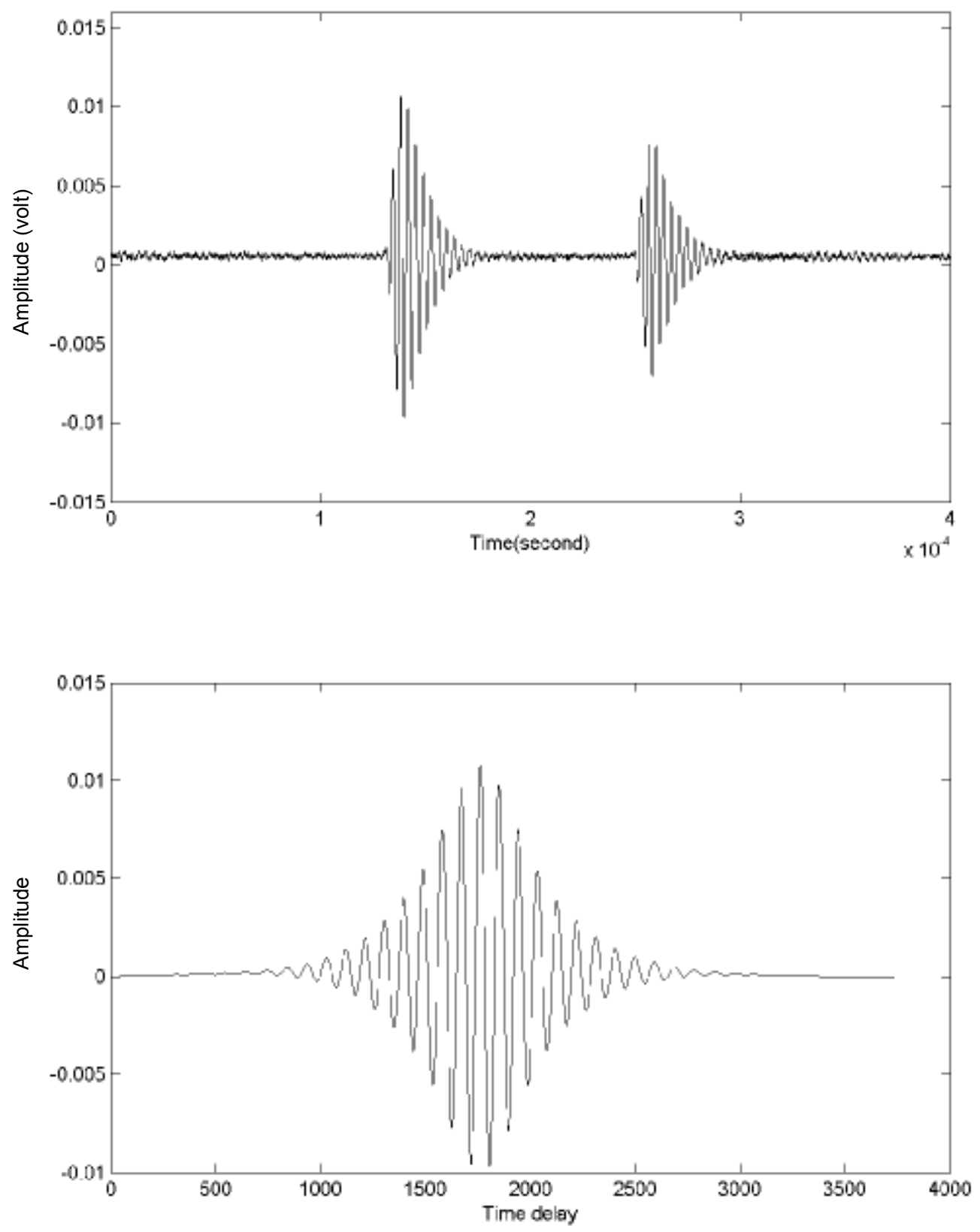

(b) Pressure: 29.66 psia

Figure A.23. Example of 279-kHz ultrasonic waveforms measured in Natural Gas \#3 and associated cross-correlation function (continued). 

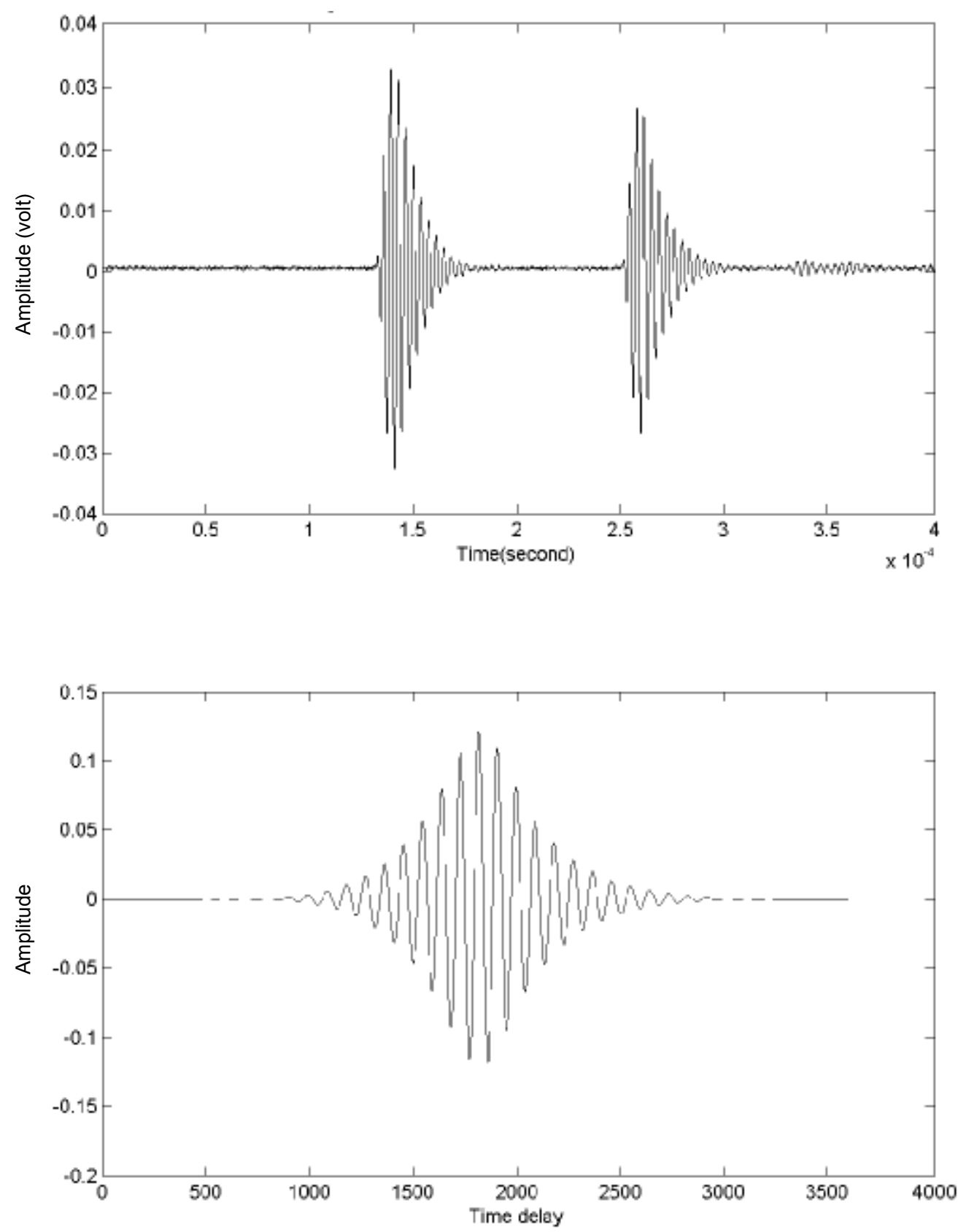

(c) Pressure: 47.54 psia

Figure A.23. Example of 279-kHz ultrasonic waveforms measured in Natural Gas \#3 and associated cross-correlation function (continued). 


\section{A.10 Summary of Bench Test Results}

A comparative evaluation of the bench tests performed on the five ultrasonic transducers indicates that the Etalon Model CIA-3525-SB (279-kHz) transducer and the Ultran Model NCT$105(500-\mathrm{kHz})$ transducer provide the most effective speed-of-sound measurements. The 279$\mathrm{kHz}$ transducer is superior to the $500-\mathrm{kHz}$ transducer in accurately measuring the speed of sound in several gas mixtures over the $20-100$ psia pressure range. In particular, under the controlled bench test conditions, the speed-of-sound sensor using the $279-\mathrm{kHz}$ transducer provided inherently acceptable measurements within the tolerance required for the energy meter application. That is, the measured speed-of-sound values were within tolerance without requiring any compensating adjustments for offset errors or other calibration. The high signal-to-noise ratio obtained with the Etalon Model CIA-3525-SB 279-kHz transducer when operating at a test pressure of 95 psia points out the improvement to be gained in acoustic coupling efficiency in comparison with lower operating pressures.

In comparison with the $279-\mathrm{kHz}$ transducer, the $500-\mathrm{kHz}$ transducer had a $0.3-0.4 \%$ offset in measured speed of sound relative to the calculated values in two different natural gas mixtures. This offset error is primarily attributed to diffraction effects associated with operating the transducer in the near field, although other experimental errors are probably also present. Nevertheless, with proper calibration adjustment, the $500-\mathrm{kHz}$ transducer could be adapted to produce accurate speed-of-sound measurements. The principal limitation of the $500-\mathrm{kHz}$ transducer is its relatively narrow bandwidth and the associated marginal amplitude distinction between its maximum coherence peak and adjacent correlation peaks. This limitation caused cycle skipping errors in automatically selecting the maximum coherence peak, resulting in incorrect determination of the differential time of flight between the reflected pulses. For this reason, the Ultran NCT-105 $500-\mathrm{kHz}$ transducer is not acceptable for use in the energy meter speed-of-sound sensor.

The Ultran Model NCT-510 (1000 kHz) transducer has a resonance frequency that is too high for efficient operation in natural gas mixtures in the pressure range used in the bench tests. However, by operating this transducer below resonance at $750 \mathrm{kHz}$, limited but usable measurements were obtained in two natural gas mixtures at the higher test pressures. The signalto-noise ratio in these measurements was low in both cases, leading to time-of-flight measurement errors caused by noisy signals rather than by limited bandwidth. The effective bandwidth in these measurements was about $350 \mathrm{kHz}$ (50\% of peak response frequency), which resulted in a compact ultrasonic pulse and a well-defined correlation peak even though the derived time of flight was distorted by the background noise. This transducer is not appropriate for use in the energy meter application because of its low sensitivity in the frequency range appropriate for precision speed-of-sound measurements in natural gas at pressures below 100 psia.

The two lower frequency ultrasonic transducers evaluated in the bench tests were found to be inadequate for use in the energy meter application because of their limited bandwidth and poorly defined ultrasonic pulse waveforms. 